\title{
Environmental Impact Directory System: Preliminary Implementation for Geothermal Energy
}

\author{
Prepared by Environment and Safety Group
}

\author{
July 1976
}

Prepared for

Office of the Assistant Administrator for Environment and Safety ENERGY RESEARCH AND DEVELOPMENT ADMINISTRATION W ashington, D.C. 20545

Contract No. E(04-3)-1101-PA4

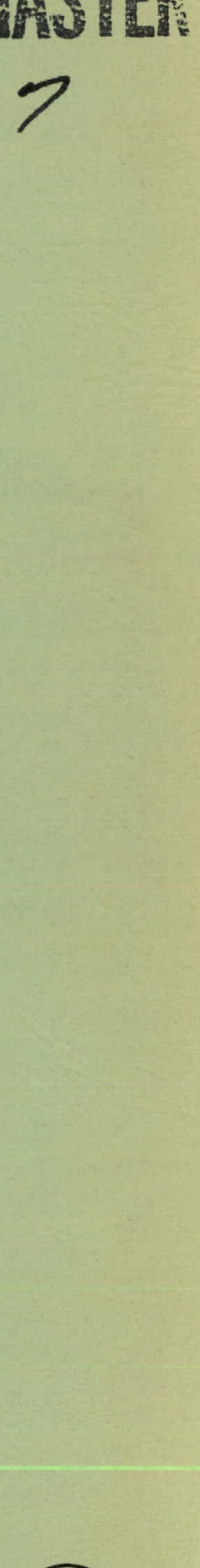

Environment and Energy Conservation Division THE AEROSPACE CORPORATION 


\section{DISCLAIMER}

This report was prepared as an account of work sponsored by an agency of the United States Government. Neither the United States Government nor any agency Thereof, nor any of their employees, makes any warranty, express or implied, or assumes any legal liability or responsibility for the accuracy, completeness, or usefulness of any information, apparatus, product, or process disclosed, or represents that its use would not infringe privately owned rights. Reference herein to any specific commercial product, process, or service by trade name, trademark, manufacturer, or otherwise does not necessarily constitute or imply its endorsement, recommendation, or favoring by the United States Government or any agency thereof. The views and opinions of authors expressed herein do not necessarily state or reflect those of the United States Government or any agency thereof. 


\section{DISCLAIMER}

Portions of this document may be illegible in electronic image products. Images are produced from the best available original document. 
This report was prepared with the support of the United States Energy Research and Development Administration, Contract Number E(04-3)-1101-PA4. However, any opinions, findings, conclusions, or recommendations expressed herein are those of the authors and do not necessarily reflect the views of ERDA. 
Aerospace Report No.

A TR-76(7518)-9

\section{ENVIRONMENTAL IMPACT DIRECTORY}

SYSTEM: PRELIMINARY IMPLEMENTATION

FOR GEOTHERMAL ENERGY

Prepared by

Environment and Safety Group

This This book was prepared as an account of nor any agency therceof, nor any of their emplovees, makes ary Neither the United Slates Gouled or assumes ony legal libbility or respon or process discloset. On warranty, express or istulness of any information. abdatus. completents.

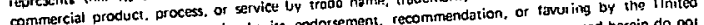
not necessarily constitute or imply its endorsenms and opinions of authors expressed herein do nol States Government of any agency thereet. The vitos Government or any agency thereat.

necessarily state or retlect thoseor

Environment and Energy Conservation Division

THE AEROSPACE CORPORATION

Germantown, Maryland 20767

$$
\text { Prepared for }
$$

Office of the Assistant Administrator for Environment and Safety ENERGY RESEARCH AND DEVELOPMENT ADMINISTRATION

Washington, D.C. 20545

Contract No. E(04-3)-1101-PA4 


\section{THIS PAGE \\ WAS INTENTIONALLY \\ LEFT BLANK}


THE ENVIRONMEN'TAL IMPACT DIRECTORY SYSTEM:

PRELIMINARY IMPLEMENTATION FOR GEOTHERMAL ENERGY

Prepared by:

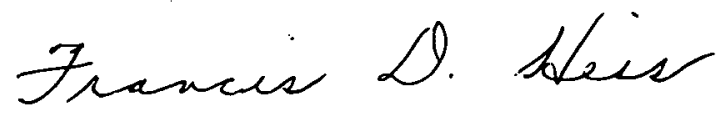

Francis D. Hess

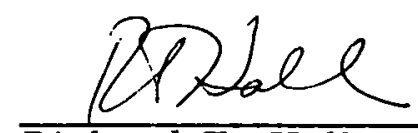

Richard T. Hall

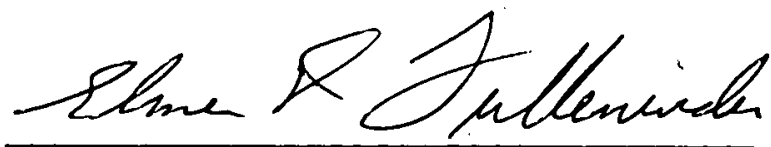

Elmer D. Fullenwider

Approved by:
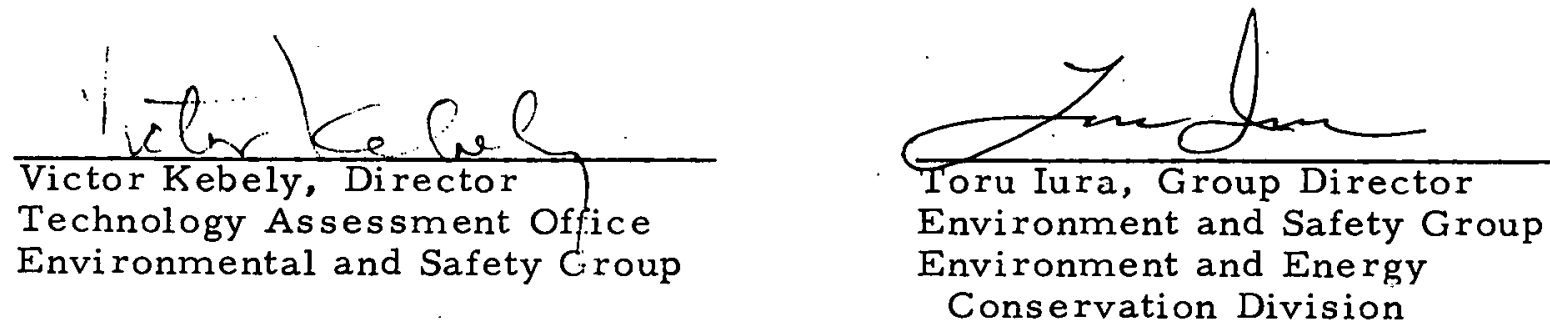


\section{THIS PAGE WAS INTENTIONALLY LEFT BLANK}


FOREWORD

This report is presented in partial fulfillment of Contract No. E(04-3)-1101-PA4 between The Aerospace Corporation and the United States Energy Research and Development Administration. It presents a preliminary implementation of the concept of the Environmental Impact Directory System for geothermal energy. A companion report entitled "The Environmental Impact Directory System: A Conceptual Description" presents a more complete description of the concept and its development.

Appreciation is expressed to Hodge R. Wasson of the Energy Research and Development Administration and to Jack O'Brien of The Aerospace Corporation for their assistance in supplying many reports related to geothermal energy. The cooperation and hospitality of the personnel at the various agencies and laboratories visited during the course of this effort is deeply appreciated.

It is intended that this report be a working document and that through its circulation comments will be forthcoming as to the value of the concept as well as the usefulness of this preliminary implementation. These comments should be directed to:

Mr. John Wilson

Office of Environmental Information Systems

Office of the Assistant Administrator for Environment and Safety

U. S. Energy Research and Development Administration

Germantown, Maryland 20767 


\section{THIS PAGE \\ WAS INTENTIONALLY \\ LEFT BLANK}


ABSTRACT

An Environmental Impact Directory System (EIDS) was proposed as a method for a computerized search of the widely distributed data files and models pertaining to energy-related environmental effects. To define the scope and content of the system, an example was prepared for the case of geothermal energy. The resulting sub-directory is known as GEIDS (Geothermal Environmental Impact Directory System).

For the complete EIDS program, the structure is that of a threedimensional matrix having a list of energy-conversion technologies as one dimension. Reducing this dimension to geothermal energy alone, a twodimensional slice of the EIDS matrix remains, consisting of energy residuals (emissions, effluents, processes) as one axis and environmental recipients (water, air, biota) as the other axis. To complete the matrix, major residuals and recipients are subdivided down to any desired level of component parts, resulting in a hierarchical tree for that particular matrix element. Then the intersection of each residual with a recipient comprises an area of a possible environmental impact. For each node of intersection the severity of impact has been estimated and a list of pertinent data bases and models provided. To expedite this preliminary sampling, some condensations have been made to reduce the number of entries to a manageable figure while preserving the overall methodology.

In preparing or reviewing an Environmental Impact Statement (EIS), the user may employ GEIDS as an extensive checklist to make sure he has taken into account all predictable impacts at any level of severity. In addition, if he must quantify an impact, he may consult the listing of available models and data bases, refer to the directory for their descriptors and abstracts, and order from the holding agency those that relate to his program. Or he may perceive gaps in the information he requires and arrange for whatever research is needed to fill them. 


\section{THIS PAGE \\ WAS INTENTIONALLY \\ LEFT BLANK}


CONTENTS

ABSTRACT $\ldots \ldots \ldots \ldots \ldots \ldots \ldots \ldots \ldots \ldots \ldots \ldots \ldots \ldots \ldots \ldots$ vii

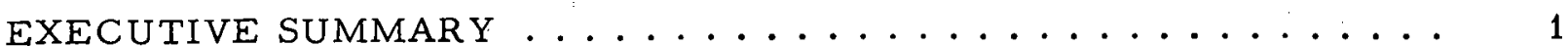

1. THE CONCEPT OF THE ENVIRONMENTAL IMPACT

DIRECTORY SYSTEM . . . . . . . . . . . . . 1-1

2. THE GEOTHERMAL ENVIRONMENTAL IMPAC'T

DIRECTORY SYSTEM - PRELIMINARY IMPLEMENTA'IION . . . 2-1

REFERENCES ....................... R 1

APPENDIXES

A. ENVIRONMENTAL IMPACT DIRECTORY SYSTEM

DIMENSION TABULATION. ................... A

B. GEOTHERMAL ENVIRONMENTAL IMPACT DIRECTORY

SYSTEM IMPACT CHECKLIST . . . . . . . . . . . . B-1

C. GEOTHERMAL ENVIRONMENTAL IMPACT DIRECTORY

SYSTEM MODEL AND DATA BASE DIRECTORY ........ C-1 
FIGURES

1-1. The EIDS Structure...................... 1-4

1-2. Geopower Dimension Tree ..................... 1.

1-3. Water Residuals Dimension Tree ................ 1-6

1-4. Biological Domain Recipient Dimension Tree .......... 1-7

1-5. Sample Directory Entry.................... 1-9

1-6. Sample EIDS Output ...................... 1-12

1-7. Technology-Residual-Recipient Chain ............. 1-13

1-8. Simulated EIDS Checklist Output ................ 1-15

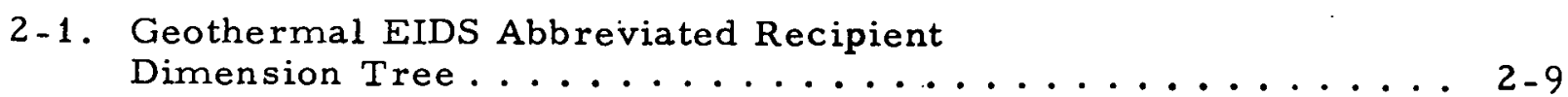

\section{TABLES}

1 1. Environmental Impact Directory System Categories . . . . . . 1-3

2-1. Dimension Tabulation for the Geothermal Environmental

Impact Directory System .................. 2-2

2-2. Triads With No Data Entries ................ 2-6

2-3. Triads With No Model Entries ................... $2-7$

$2-4$ Triads With No Impact Entries ................. 2...10 


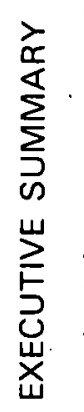

D 


\section{EXECUTIVE SUMMARY}

An Environmental Impact Directory System (EIDS) has been proposed to facilitate the preparation and review of Environmental Impact Statements (EIS) on energy-related programs. To determine the magnitude of the task and to evolve a format, a small-scale version was prepared, limited to geothermal energy. This is known as GEIDS (Geothermal Environmental Impact Directory System).

The parent system is structured as a computer-accessed matrix having three dimensions labeled technology, residuals, and recipients. The nine technology headings include those methods of power generation and energy conservation for which ERDA has responsibility in the research, development, and demonstration sectors. Since geothermal energy is one of these technologies, it represents a two-dimensional slice of the EIDS matrix.

Residuals, comprising one axis of GEIDS, are defined as byproducts - pollutants, effluents, and impact-producing outputs--that result from an activity in addition to its primary product. Nine major residuals are listed under geothermal energy, including liquid and gaseous emissions as well as operations affecting social, cultural, and economic changes. Likewise there are four major recipients: these are the physical-chemical domain, the biological domain, the social-cultural domain, and the economic domain. Both residual and recipient categories can be subdivided down to any taxonomic level desired, giving a hierarchical tree having as many as six levels in the example selected. However, since the intent was to demonstrate the structure of EIDS rather than provide a complete listing, no attempt was made to fill in all branches of the trees down to the same level of detail.

The impacts of residuals upon recipients may be regarded as definable for each node or axis intersection. For each intersection, an evaluation is made of the severity of impact--severe, moderate, minor, zero, unassessed--as it applies to the stage of exploitation of the technology. 
In addition, a listing is provided of those models and data bases, available within ERDA or from other sources, that provide the GEIDS user with chemical analyses; physical measurements, inventories of flora and fauna, demographic characteristics, and programs for forecasting diffusion of pollutants, or economic advantages of a technology. Second and higher order impacts may be traced as well, since a recipient's place in the ecology makes it a residual, in a sense, interacting with other recipients.

To obtain the models and data bases relevant to his work, the user is provided with a directory (Appendix C) with some 200 listings. Each one shows the following information to the extent to which it is known:

Title

Date

Geographic Area

Geographic Coordinates

FIPS (Federal Information Processing Standards)

Locator Code

Abstract

Assessment

Analyses Performed (Usage)

Funding

Model/Data Base Availability

Archive Medium (Tape, Punched Card, etc.)

Contact

Citations (Locations within GEIDS)

As in the case of the ehecklist, no attempt was made to complete all items in the model/data base directory entries. In this respect, the greatest need is for the completion of the critical assessments, to provide the user with an estimate of the validity of his data.

Figures and tables in this report illustrate the following features:

Categories of Technologies, Residuals, Recipients

EIDS Matrix 
Dimension Trees for Technologies, Residuals, Recipients

Example of Data Base Information

Technology-Residual-Recipient Chain

Simulated EIDS Checklist Output (Printout)

Dimension Tabulation for GEIDS

Triads with No Data Entries (Information Gaps) 
I 


\section{SECTION 1}

\section{THE CONCEPT OF THE ENVIRONMENTAL IMPACT DIRECTORY SYSTEM}

The responsibilities of ERDA for the preparation of Environmental Impact Statements (EIS) are clearly defined in the NEPA Section 102(C). The content of the EIS is further outlined in Executive Order 11514 and in Section 1500.8 of the Council on Environmental Quality (CEQ) Guidelines. Beyond these requirements, ERDA has a great responsibility in the selection of - alternative energy sources, for once selected, they will establish the pattern of energy-related environmental impacts for many years to come. Consequently, ERDA needs the most relevant and reliable environmental information available. It must have a ready means of determining what information is appropriate to a specific project, where that information is located, and what constraints may exist on its reliability or scope. The Environmental Impact Directory System (EIDS) is proposed to meet these needs.

The Environmental Impact Directory System has as its basis the notion

\section{A Technology in producing energy also produces a Residual that acts upon a Recipient.}

The concept of a technology needs no elaboration; howèner, residuals and recipients do. Residuals are defined in the sense of the University of Oklahoma's Energy Alternatives:

"Residuals" are defined as the byproducts that an activity, technological alternative, or process produces in addition to its primary product. Using this broad definition, residuals include such effects as air and water pollutants, solid wastes, and impact-producing inputs (e.g., the materials requirements of a particular process) (Ref. 1). 
As can be seen from this definition, the term "residual" is broader than and includes what are classicially considered "pollutants." This expansion of terms is necessitated by the desire to include the whole range of consequences of a technology. Recipients are whatever the residual acts upon. Again this definition is broader than what is normally considered with the term "impact." This was necessitated by the desire to include the whole range of areas of fields a residual could impact.

Using these definitions, there are nine broad categories of Technnologies, nine of Residuals, and four of Recipients, as defined in Table 1-1. These three sets of categories can be thought of as the "dimensions" of an environmental impact. The dimensions then can be thought of as forming a three-dimensional matrix or volume such as is shown on the right of Figure 1-1. Each cube in the volume is then a different combination of Technology, Residual, and Recipient.

Each one of these broad categories can be broken down in detail to whatever level is desired. The result is a set of dimensional trees. Examples of these trees are given in Figures 1-2, 1-3, and 1-4.

Figure $1-2$ is a dimensional tree for the geopower technology. There are four subdivisions of geopower given. The ellipses indicate additional subdivisions could be added. Each of these subdivisions can be further broken down. In the example, the geothermal energy is broken down into four subcategories. This process can continue to whatever level of detail is desired. In the example there are four levels of detail. In addition, for the Technology dimension, there is an operational breakdown appended to whatever detail level one is working at. This operational breakdown is given at the bottom of the figure. For example, two of the many possible chains within the Technology dimension tree that can be traced are:

Technology: Geopower: Geothermal Finergy: Hydrothermal

systems: Hot-water systems: High temperature: ...

Technology: Geopower: Hydroelectric power: ... 
Table 1-1. Environmental Impact Directory System Categories

$\underline{\text { Technology }}$

Fossil

Nuclear

Geopower

Solar Energy Conversion

Energy Conservation

Magnetohydrodynamic

Power

Energy Storage

Thermoelectric Power

Therminoic Power $\underline{\text { Residual }}$

Earth Residuals

Water Residuals

Atmospheric Residuals

Biological Residuals

Social-Cultural Residuals

Economic Residuals

Public Health Residuals

Infrastructure Residuals

Catastrophic Residuals $\underline{\text { Recipient }}$

Physical and Chemical Domain

Biological Domain

Social-Cultural

Domain

Economic Domain

After the final colon in each chain, the appropriate operational phase should be added. Figure 1-3 shows a six-level-of-detail breakdown of the water residuals dimension tree. Similarly, Figure 1-4 shows a four-level-of-detail breakdown of the biological domain recipients dimension tree.

The concept of the level-of-detail breakdown is illustrated graphically on the left side of Figure 1-1. For example, one can start with two cubes of the basic volume corresponding to the nuclear technology, the physical and chemical domain recipient, and the earth and water residuals. These two cubes were firstbroken down along the technology axis into fusion and fission and finally breeder and non-breeder reactors. The non-breeder reactor. slice was then broken down along the residual and recipient axes, resulting in the volume at the left of the figure. A tabulation of the current version of the dimensional trees is given in Appendix A. The trees in the dimensional tabulation obviously have not been uniformly traced out to equivalent levels of detail or depth over their entire structures. The great detail in some 


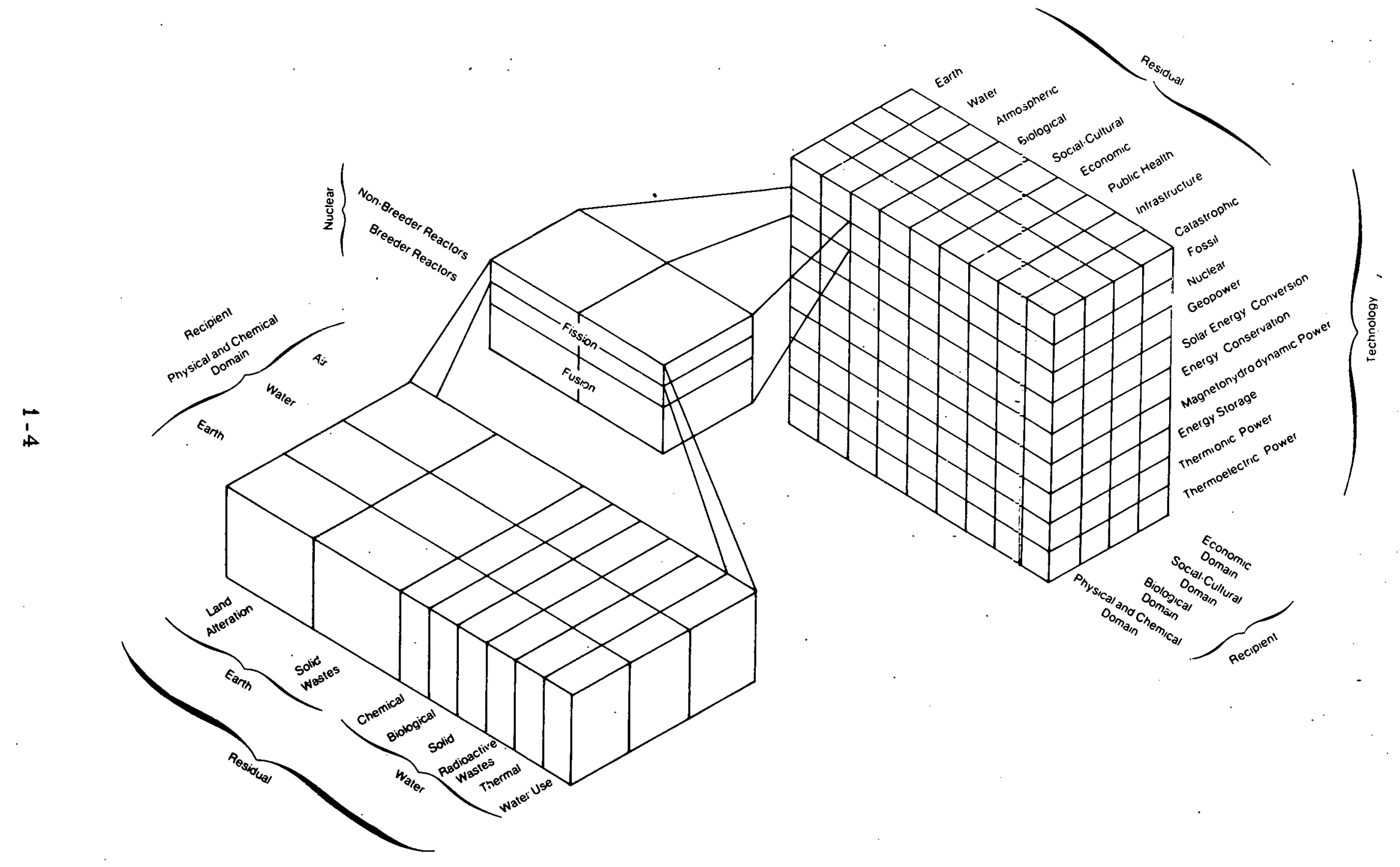

Figure 1-1. The EIDS Structure 


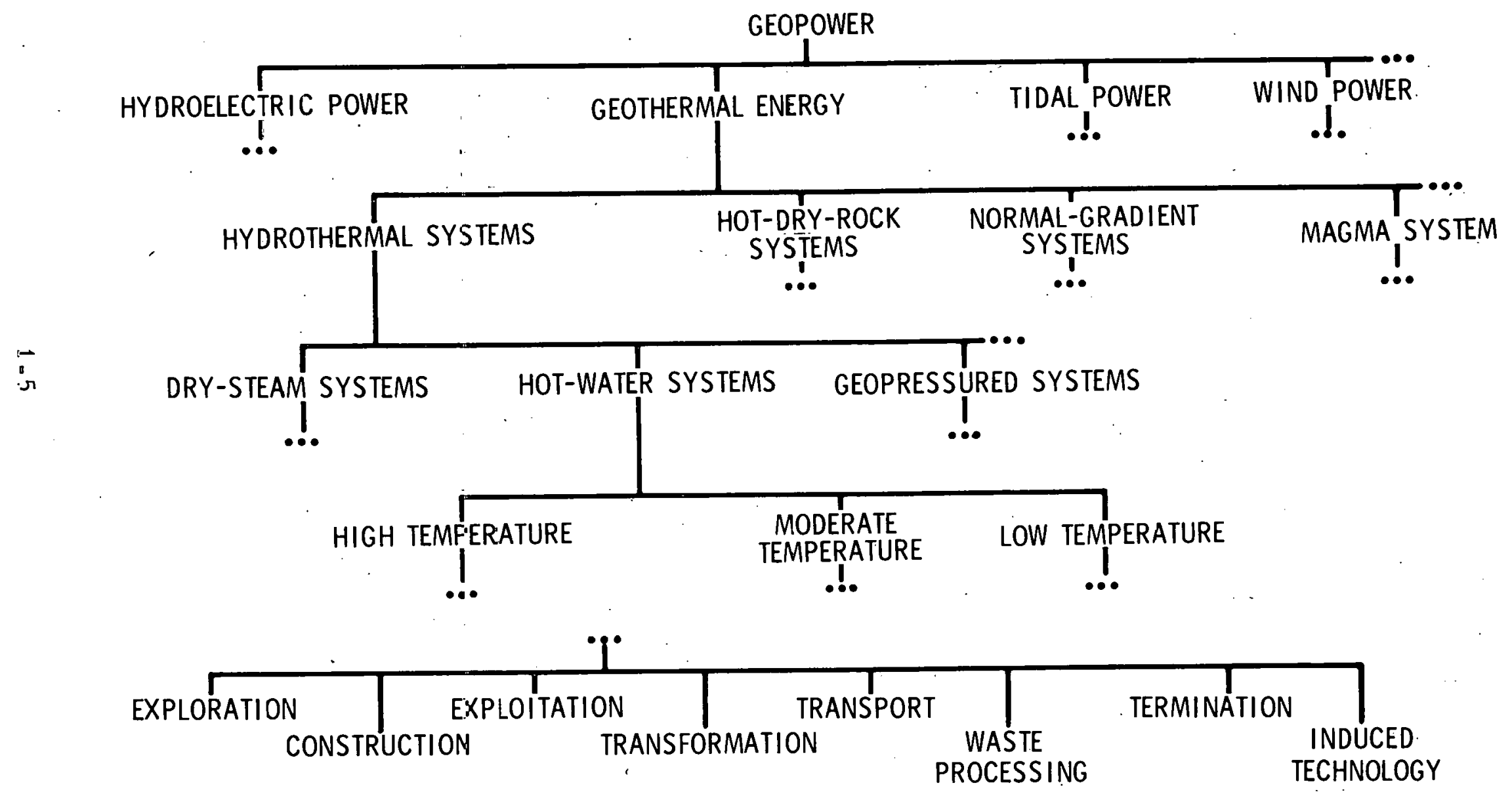

Figure 1-2. Geopower Dimension Tree 


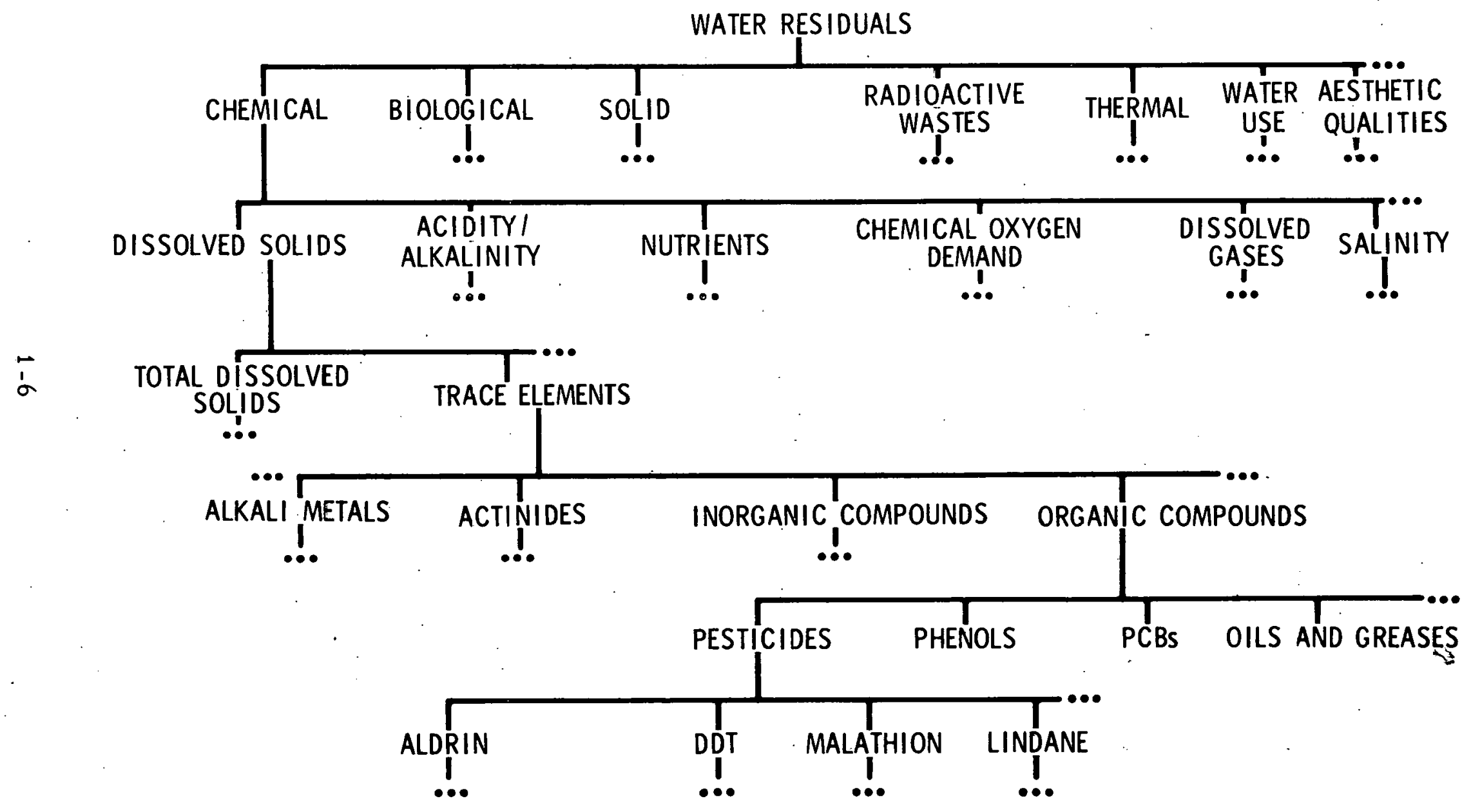

Figure 1-3. Water Residuals Dimension Tree 


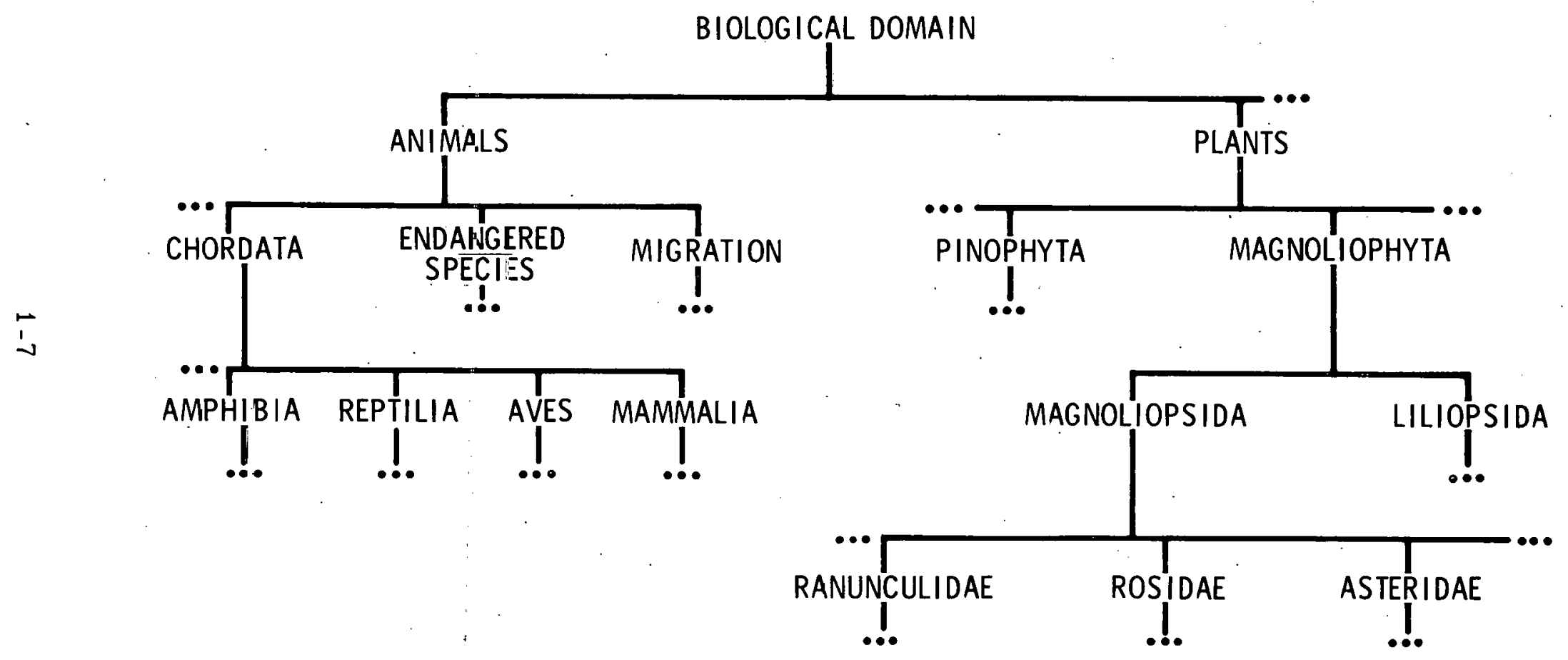

Figure 1-4. Biological Domain Recipient Dimension Tree 
areas is not meant to indicate a greater concern or priority for those areas. Rathe $r$, the detail in certain areas was intended to demonstrate how the EIDS structure could handle such detail. The nomenclature in the dimensional tabulation is based upon, as far as possible, terms found in the ERDA Thesaurus (Ref. 2). Where no equivalent term was found in the Thesaurus, the term in the dimensional tabulation has an asterisk. If, in later revisions of the ERDA Thesaurus, appropriate terms are introduced, these asterisked terms may be changed. This version is not intended to be complete or final but rather is subject to expansion and revision according to user's comments.

Thcse three-dimensional trees form the basic structure of the EIDS. The one additional feature of the EIDS is the directory of environmental models and data bases. A typical proposed format of an entry in this directory is shown in Figure 1-5. Each entry in the directory consists of a basic set of information:

1. Data base or model entry code number. This is an internal reference code used to refer to the data base or model within the EIDS structure.

2. Title. This is the title of the data base or model.

3. Geographic area. This is a hierarchical textual description of the geographic area of the data or modeled area. The hierarchy is regional (e.g., Mid-Atlantic states), state (e.g., Maryland), local (e.g., Potomac River). The description will allow text searching for applicable models or data bases.

4. Geographic coordinates. This is simply the latitude and longitude of the data or modeled area and will allow numeric searching for applicable models or data bases.

5. The FIPS code. This is the Federal Information Processing Standards code of states and counties (Ref. 3). Standard Metropolitan Statistical Areas (SMSAs) could also be coded here (Ref. 4). The code allows numerical searching by area for applicable models or data bases.

6. Abstract. This brief abstract tells what, where, and when the measurements or models were made. 
Data Base: BLS/DF-73-007

Title: Current Employment Statistics Program

Date: 1939 to date

Geographic Area: National, states, areas (mostly SMSA's)

Geographic Coordinates: not applicable

FIPS Code: all

Abstract: The current employment statistics program generates state and area statistics as well as the national series. State agencies prepare state and area statistics monthly, selecting for publication those industries which best reflect currently significant economic activities. This file contains annual averages only for about 18,000 monthly time series of industry employment (all employees), and (1) average weekly earnings; (2) average weekly hours, and (3) average hourly earnings of production workers in states and selected areas (mostly SMSA's). There are approximately 325,000 variable length records. The format was originally designed for IBM 7070 series computers.

Assessment: none

Analyses Performed: "Employment and Earnings" (monthly publication)

"Monthly Labor Review" (monthly publication)

Funding: Bureau of Labor Statistics

Data Availability: National Technical Information Service Computer Products Office

Springfield, VA 22151

Archive Medium: 6 reels of 7 -track, 556 bpi, BCD-code magnetic tape

Contact: Bureau of Labor Statistics

Wa shington, DC

Master Control No. 60471

Citations: 1.3.2--2.6--3.4.1

Figure 1-5. Sample Directory Entry 
7. Assessment. This is an independent assessment of the accuracy, sensitivity, and region of applicability. Included here would also be any general peer review comments. This is an important feature of the EIDS but one needing the greatest care and effort in its implementation.

8. Analyses performed. This is a citation of any published reference to analyses performed using the data or model.

9. Funding. This tells for whom the work was carried out and is particularly useful in keeping track of ERDA-funded efforts.

10. Data availability. This tells the user if the data or model is available and at what cost.

11. Archive medium. This tells in what medium (e.g., magnetic tape, computer cards, tabular, etc.) the data or model is stored. It could have a great bearing on the usefulness of a particular data set.

12. Contact. This tells the user whom to contact to find out more about the data base or model. It, too, is a very important feature of the EIDS and will require considerable effort to ensure its accuracy and timeliness. It is an upto-date version of the card files of contacts many preparers of environmental impact statements maintain.

13. Parameter list. This is a listing of the physical parameters being measured or modeled.

14. Citations. The final basic entry in each directory entry is a listing of those dimensional triads in the EIDS structure that have cited this particular data base or model. The citation is helpful in furthe $r$ assessing the contents of the data base or model. It only gives the bottom member of any tree. Obviously, all higher members of the tree will also have this citation.

Although these 14 items form the basic directory entry, additional items, as appropriate, could be added.

The basic concept of the EIDS and how it is used are illustrated by the following examples. Persons involved in the process of environmental assurance would use the EIDS in one of two ways. In one way the user queries the EIDS directly at a particular dimension triplet of technology, residual, 
and recipient at any level of detail on the three-dimension trees. The EIDS will give, for this particular triplet, the estimated maximum potential environmental impact, examples of the impacts, citations of available data, and environmental models applicable to the triplet. In addition, citations to existing environmental analyses applicable to the triplet are given. A sample of a suggested output format is given in Figure 1-6. Once the user has determined that the potential entironmental impact is applicable to the particular situation with which he is concerned, he can query the directory for the data and model citations. This latter point is important. The EIDS is designed to give estimated environmental impacts on the technology worst-case basis. In estimating these environmental impacts, uncontrolled or at least worse-than-average controlled technology is assumed. The application of standard control technology would mitigate many of these impacts. Obviously, if the estimated impact example is concerned with some coastal estuary and the user is interested in a desert site, this particular impact can be ignored.

There is an aspect of the estimated impacts that should be amplified. Figure 1-7 shows a possible set of interrelated residuals resulting from a particular residual. These are the second and higher order environmental impacts. In the EIDS structure and concept, each of the se four recipients would have an estimated environmental impact entered at its dimension triad with the appropriate technology and residual. In a sense, each recipient in the chain becomes the "residual" for the next link. This is an important feature of the EIDS concept.

Once the user has queried the system at a particular dimensional triplet, he can determine related environmental impacts by holding any two of the three dimensions constant and search along the resulting line. This search could be manual or computer-assisted depending on the actual hardware configuration of the EIDS. In this way the user can be alerted to potential higher order impacts. 
Technology: $\quad 1.3 .2$

Geopower

Geothermal Energy

Residual: $\quad 2.2$

Water Residuals

Recipient: 3.1 .1 .2

Physical and Chemical Domain

Earth

Resources

Potential Environmental Impact: A. Exploration MINOR

B. Construction MODERATE

C. Exploitation SEVERE

D. Transformation MINOR

E. Transport MINOR

F. Waste disposal/processing SEVERE

G. Termination MODERATE.

H. Induced technology UNASSESSED

Example Impacts: 1. C. Withdrawal of Reservoir Water Depletes Geothermal Resource

2. F. Reinjection of Brine May Clog Aquifer

Data Available: 1. IVEP

. 2. GRID

3. GEOTHERM

Models Available: 1. IGS/CARTWRIGHT

2. USGS/FOURNIER

Figure 1-6. Sample EIDS Output 
TECHNOLOGY

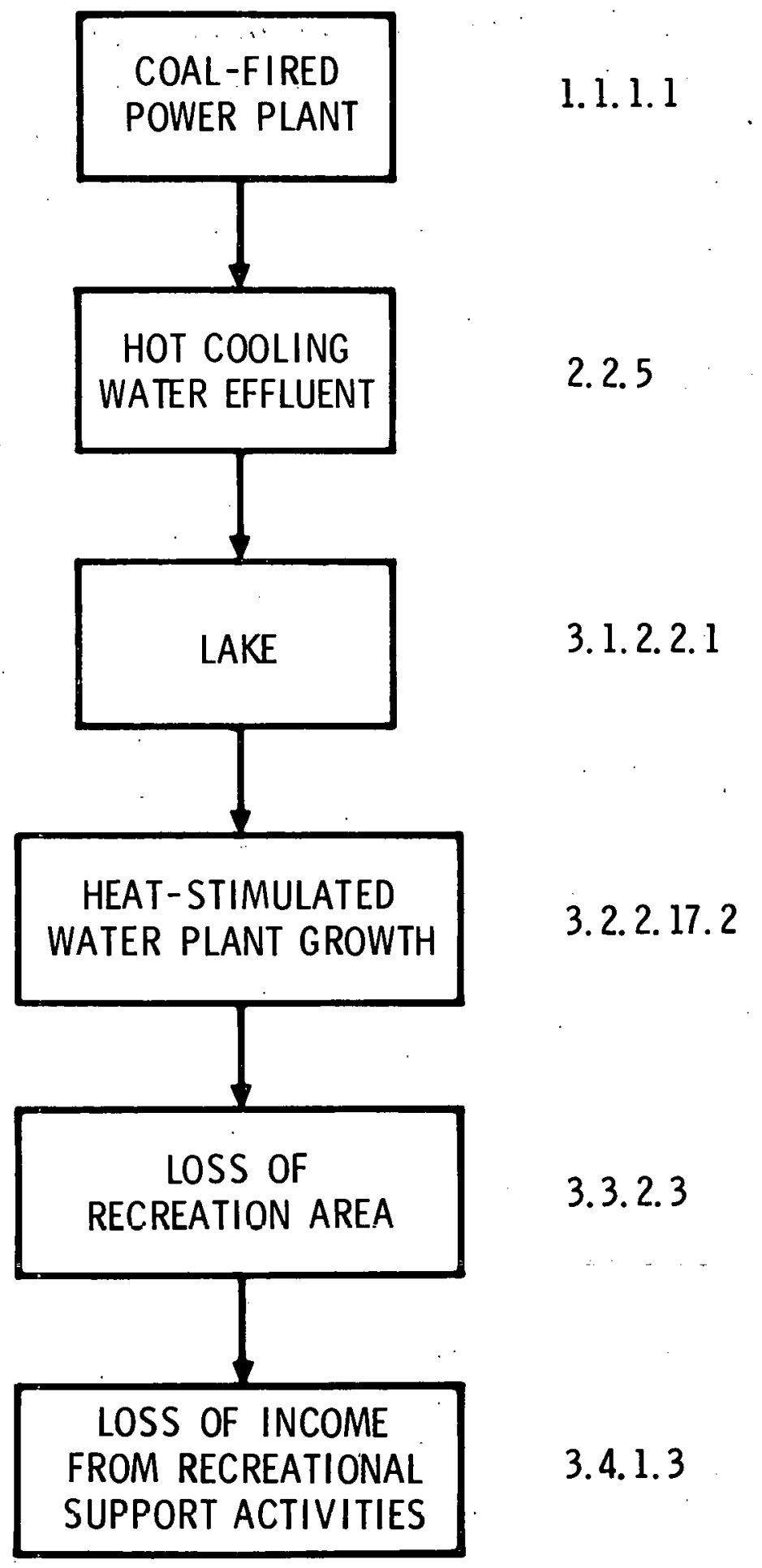

Figure-1-7. Technology-Residual-Recipient Chain 
The second mode of operation of the EIDS is as a checklist. In this mode the user takes slices through the basic structure along any of the three axes. This is equivalent to holding one dimension constant and allowing the othe $r$ two to vary over their range. For example, holding the technology dimension constant will give all the possible combinations of residuals and recipients for that technology. Similarly, holding the recipient dimension constant will give all the possible combinations of technologies and residuals. The resulting slices can then be searched for estimated potential impacts above a certain threshold, for example, only "severe" impacts. The result of this search is a checklist of combinations of the two free dimensions that have potential environmental impacts of a given severity or greater. The output could also include citations to applicable data or models. A suggested sample format of the checklist output of the EIDS is given in Figure 1-8.

Such a checklist output would be particularly useful to the reviewer of environmental analyses. The checklist would indicate those impacts that should have been covered in the analysis. 


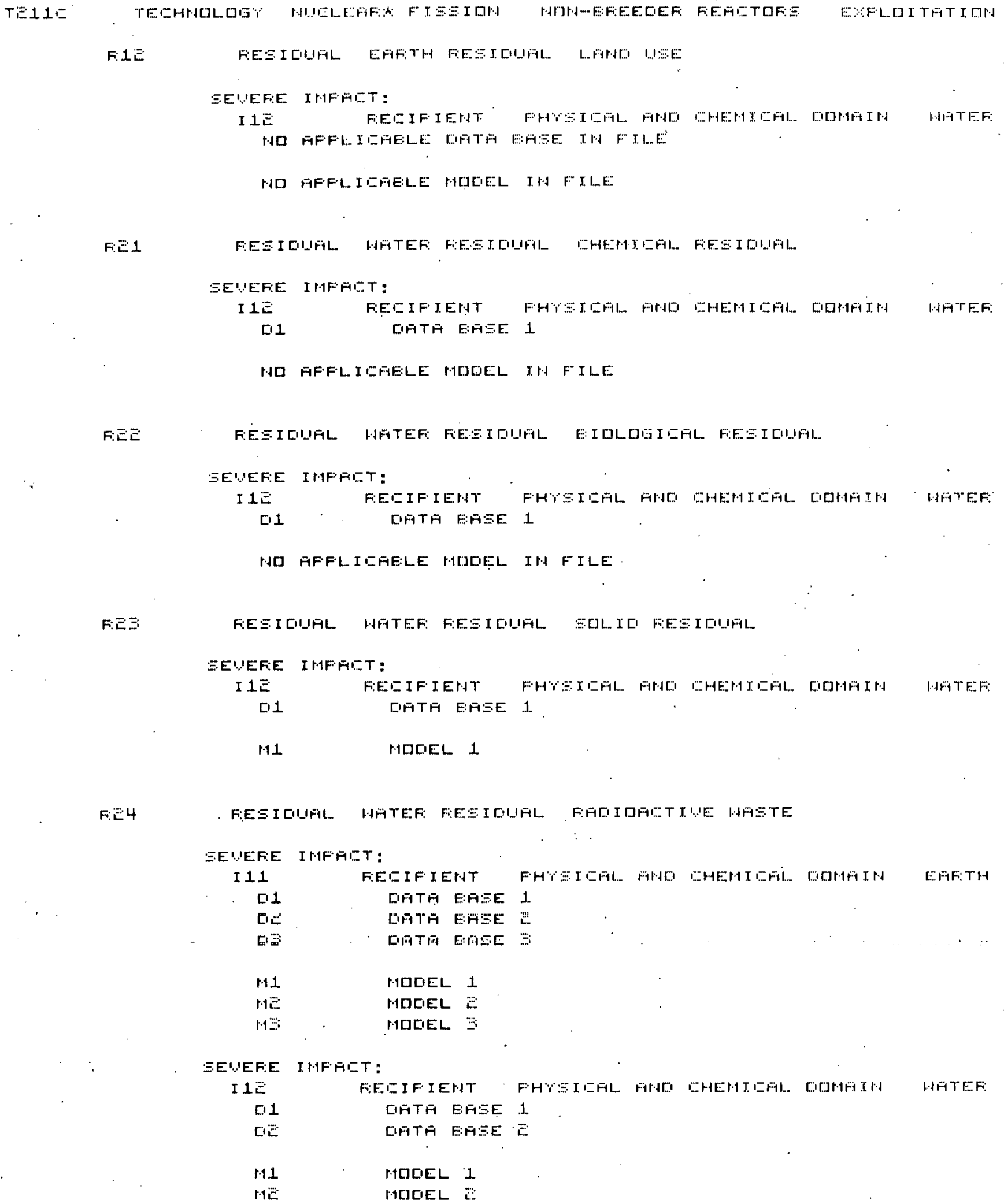

Figure 1-8. Simulated EIDS Checklist Output 
0

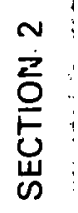




\section{SEC TION 2}

\section{THE GEOTHERMAL ENVIRONMENTAL IMPACT DIRECTORY SYSTEM - PRELIMINARY IMPLEMENTATION}

In order to clarify the structure and implementation of the Environmental Impact Directory System and to offer an insight into the scope of the entire system, it was decided, upon consultation with personnel of ERDA/OAES, to construct an incremental portion of the EIDS for one of the smaller technologies. The area of geothermal energy was chosen because of ready access to the necessary data.

Because of limitations of time and manpower, several steps were taken to simplify this effort. Most importantly, the critical assessments of the validity of the environmental models and data bases were carried out only in a cursory fashion. In the EIDS concept, these as sements should be made by experts in the respective fields somewhat akin to the pee $r$ review process to which scientific publications are subject.

Secondly, the dimensional trees were much simplified. Table 2-1 gives the abbreviated dimension tabulation used in this sample. It was felt that rather than have a large number of entries with no data or model entries it would be better to coalesce the dimensional trees. Even this abbreviated version has 324 dimensional triads.

The preliminary implomentation of the Geothermal Envirominental Impact Directory System (GEIDS) was conducted in the following manner. Several broad reviews of geothermal energy developments were studied and discussions with experts in the field of geothermal energy were held. The potential maximum environmental impact of each of the 324 dimensional triads was then estimated. These estimates were made on a scale of zero to four. Zero corresponds to no impact, one to a minor impact, two to a moderate impact, three to a severe or major impact, and four to an 
Table 2-1. Dimension Tabulation for the Geothermal Environmental Impact Directory System

Technology

$$
1.3 .2
$$

Geothermal energy

Residuals

2. 1

2. 2

2.3

2. 4

2.5

2.6

2.7

2.8

2.9

Recipients

3. 1

3. 1.1

3.1.1.1

3. 1.1 .2

3. 1. 1.3

3. 1.1 .3 .1

3.1.1.3.2

3.1 .1 .3 .3

3. $1.1 .3: 4$

3. 1.1 .3 .5

3. 1.1 .3 .6

3. 1. 1.4

3. 1.1 .4 .1

3. 1.1 .4 .2

3. 1.1 .4 .3

3. 1.1 .4 .4

3. 1.1 .4 .5

3.1 .1 .4 .6

3. 1.1 .5

3. 1.1 .5 .1

3. 1.2

3.1 .3
Earth residuals

Water residuals

Atmospheric residuals

Biological residuals

Social-Cultural residuals

Economic residuals

Public health residuals

Infrastructure residuals

Catastrophic residuals

Physical and Chemical Domain Earth

Topography

Resources

Slow processes

Weathering

Solution

Sorption/Complexing

Deposition

Compaction

Subsidence

Rapid processes

Flood

Erosion

Collapse

Land slide / Avalanche

Seismicity

Air movement

Unique features

Aquifer recharge

Water

Air 
Table 2-1. Dimension Tabulation for the Geothermal Environmental Impact. Directory System (Continued)

3.2

3. 2.1

3. 2.2

3.3

3. 3.1

3. 3.2

3. 3.3

3. 3.4

3. 3.4 .1

3. 3.4 .2

3. 3.4 .3

3. 3.5

3.4

3. 4.1

3. 4.2
Biological Domain

Animals

Plants

Social-Cultural Domain

Land use

Recreation

Aesthetics

Quality of life

Health and safety

Life style

Population characteristics

Legal aspects

Economic Domain

Demographic factors

Infrastructure domain 
unassessed impact. While these terms are subjective, they we re applied uniformly to each dimensional triad. They should not be interpreted as hard and fast but are the best estimates possible based on data available in the time frame of this effort.

Once the impacts were estimated, examples of these impacts were drawn up. These again are based on the above-mentioned broad reviews of geothermal energy. These sample impacts should also be treated as educated guesses.

Filling in the data and model entries was a more involved undertaking. Much of the information on models and data bases derived from our preliminary survey work could be applied to the GEIDS. In addition, literature searches were carried out primarily using the ERDA RECON system. Finally, an attempt was made to tap the vast compilation, "National Index of Data Bases and Models," of the Lawrence Livermore Laboratory. This effort and its results are described in a separate document entitled, "An Application of the EIDS Dimension Structure to the 'National Index of Ene rgy and Environmentally Related Data Bases and Models'." Briefly, this memo describes the "National Index" and the "Master Control Program" used to access it. The "National Index" contains citations very similar in content to those found in the EIDS Directory. It contains 4103 data bases and 4595 models covering a vast range of subjects. These entries are accessed by the "Master Control Program," which has a very powerful capability for accessing mass storage files according to character strings. It was designed to overcome the limitations of a rigid keyword or hierarchical structure. Unfortunately, this great flexibility can also be a liability because the lack of a consistent thesaurus of terms makes subject searching difficult for two reasons. First, the user must decide which of many synonymous terms he will search on (multiple terms can be used). For example, should he search for "petroleum," "fossil fue1," "oil," or what? Second, unless the search terms explicitly appear in the same part of the citation, the re will be no match. The "National 
Index" was searched for many of the terms in Table 2-1 plus whatever synonyms were felt appropriate. This resulted in 46 of the 122 entries in the GEIDS Directory. It is not known how many entries were missed because of the problems mentioned above. The memo concludes by recommending that an EIDS-like dimensional structure be overlaid onto the "National Index" to improve its accessibility.

The Geothermal Environmental Impact Directory System follows the basic concept of EIDS described above. Each dimensional triad is printed on a separate sheet ordered by residual and within residual by recipient. These will be found in Appendix B. Table $2-2$ is a listing of those dimensional triads for which there were no applicable data bases found in this study. Table $2-3$ is a similar listing for models. The listings a re given in a shortened style. There is an implied listing of all lower points on the dimensional tree, if such lower points exist. For example, Table $2-2$ says the re is no data base for triad 1.3.2 - 2.1 - 3.3.4. This implies that $1.3 .2-2.1-3.3 .4 .1,1.3 .2-2.1-3.3 .4 .2$, and $1.3 .2-2.1$ 3.3.4.3 also have no data base entries. However, the listing of $1: 3.2$ 2.1 - 3.1.1.3.4 is complete since there are no lower points on the dimensional tree. Figure $2-1$ is a graphical display of the recipient dimension tree as abbreviated in Table 2-1. The Directory of models and data bases follows the impact set. These will be found in Appendix C. For completeness, Table 2-4 lists triads with no impact entries. It cannot be emphasized too strongly that this GEIDS is incomplete and subject to revision upon expert review.

It is intended that this Geothermal Environmental Impact Di rectory System will serve two purposes: first, that it be of use to those preparing environmental assessments of geothermal energy, and second, that it be an accurate demonstration of the EIDS concept so that unforeseen flaws can be corrected before furthe $x$ implementation takes place. 


\section{Table 2-2. Triads With No Data Entries}

$$
\begin{aligned}
& 1.3 .2-2.1-3.1 .1 .3 .4 \\
& \text { 3.1.1.3.6 } \\
& \text { 3.1.1.4.5 } \\
& \text { 3.1.1. } 5 \\
& \text { 3. } 3.2 \\
& \text { 3. } 3.3 \\
& \text { 3. } 3.4 \\
& 1.3 .2-2.6-3.1 .1 .1 \\
& \text { 3. } 1.1 .3 \\
& \text { 3. } 1.1 .4 \\
& \text { 3.1.1.5 } \\
& \text { 3.1. } 2 \\
& \text { 3. } 2 \\
& \text { 3. } 3.1 \\
& \text { 3. } 3.2 \\
& -2.2-3.1 .1 .1 \\
& \text { 3.1.1.3.5 } \\
& \text { 3.1.1.4.3 } \\
& \text { 3.1.1.4.4 } \\
& \text { 3. } 1.1 .4 .6 \\
& \text { 3. 3. } 4.2 \\
& \text { 3. } 3.4 .3 \\
& \text { 3. } 3.3 \\
& \text { 3. } 3.4 .1 \\
& \text { 3. } 3.4 .2 \\
& -2.7-3.1 .1 \\
& \text { 3. } 3.1 \\
& \text { 3. } 3.2 \\
& \text { 3. } 3.3 \\
& -2.3-3.1 .1 .1 \\
& \text { 3.1.1. } 2 \\
& \text { 3.1.1. } 3 \\
& -3.1 .1 .4 \\
& \text { 3.1.1.5 } \\
& 3.4 .2 \\
& \text { 3. } 3.4 .2 \\
& \text { 3. } 3.4 .3 \\
& 3.4 \\
& -2.8-3.1 \\
& \text { 3. } 2 \\
& \text { 3. } 3.3 \\
& -2.4-3.1 .1 \\
& \text { 3. } 1.3 \\
& 3.3 \\
& \text { 3. } 4 \\
& \text { 3. } 3.4 \\
& \text { 3. } 3.5 \\
& -2.5-3.1 .1 .3 \\
& \text { 3. 1. } 1.4 \\
& \text { 3.1.1.5 } \\
& \text { 3. } 1.2 \\
& \text { 3. } 1.3 \\
& \text { 3. } 3.3 \\
& \text { 3. } 3.4 .2 \\
& 3.4 .2 \\
& -2.9-3.1 .1 .1 \\
& \text {.3. } 1.1 .2 \\
& \text { 3. } 1.1 .3 \\
& \text { 3.1.1.4.1 } \\
& \text { 3.1.1.4. } 2 \\
& \text { 3.1.1.4.3 } \\
& \text { 3. } 1.1 .4 .4 \\
& \text { 3. } 1.1 .4 .6 \\
& \text { 3.1.1. } 5 \\
& \text { 3. } 1.2 \\
& \text { 3. } 2 \\
& \text { 3. } 3 \\
& 3.4
\end{aligned}
$$


Table 2-3. Triads With No Model Entries

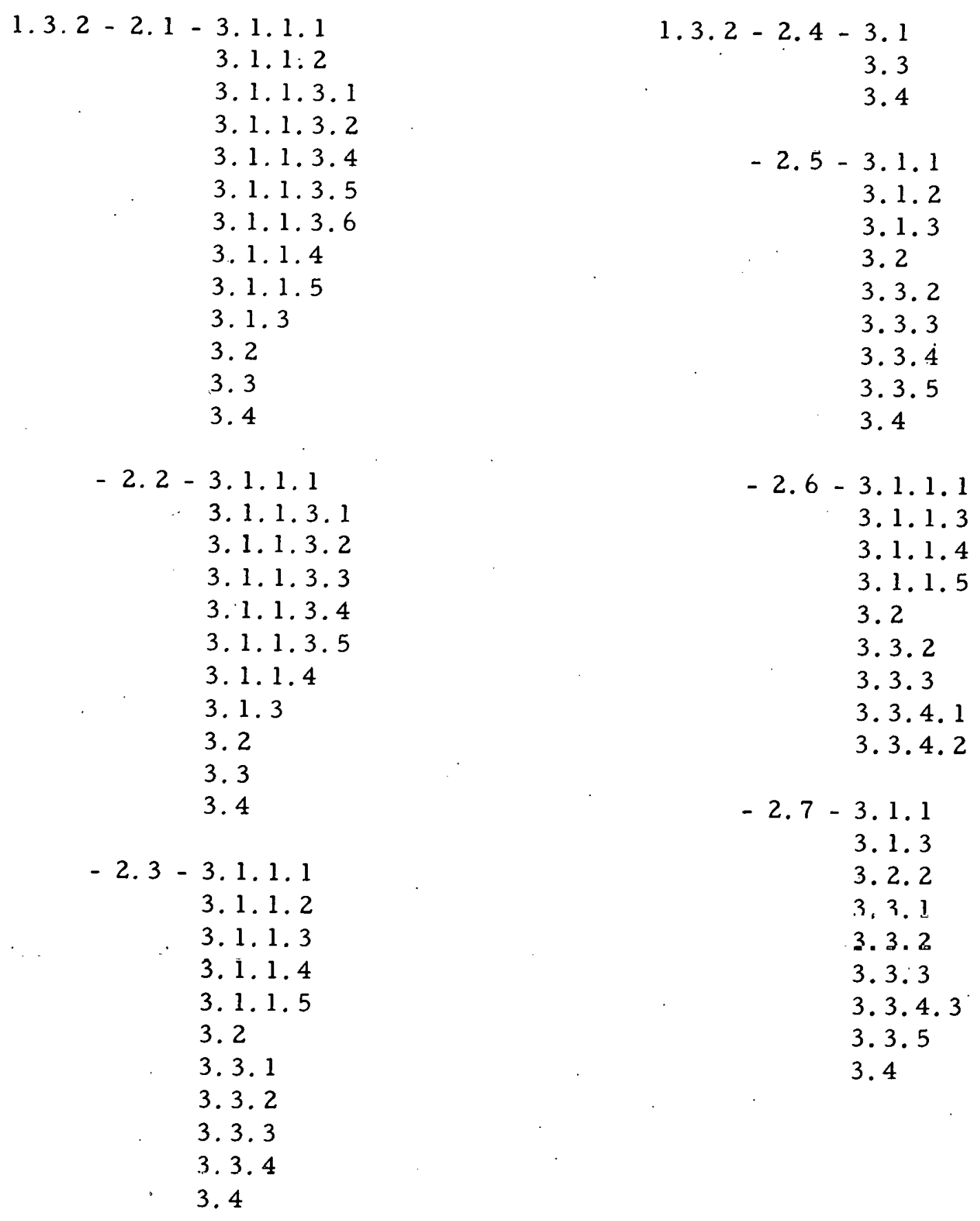

$n$ 
Table 2-3. Triads With No Model Entries (Continued)

$\begin{array}{cc}1.3 .2-2.8-3.1 .1 .1 & 1.3 .2-2.9-3.1 .1 .1 \\ 3.1 .1 .2 & 3.1 .1 .2 \\ 3.1 .1 .3 & 3.1 .1 .3 \\ 3.1 .1 .4 .1 & 3.1 .1 .4 .1 \\ 3.1 .1 .4 .2 & 3.1 .1 .4 .2 \\ 3.1 .1 .4 .3 & 3.1 .1 .4 .3 \\ 3.1 .1 .4 .4 & 3.1 .1 .4 .4 \\ 3.1 .1 .4 .6 & 3.1 .1 .4 .6 \\ 3.1 .1 .5 & 3.1 .1 .5 \\ 3.1 .2 & 3.1 .2 \\ 3.2 & 3.1 .3 \\ 3.3 .2 & 3.2 \\ 3.3 .3 & 3.3 \\ 3.3 .4 & 3.4\end{array}$



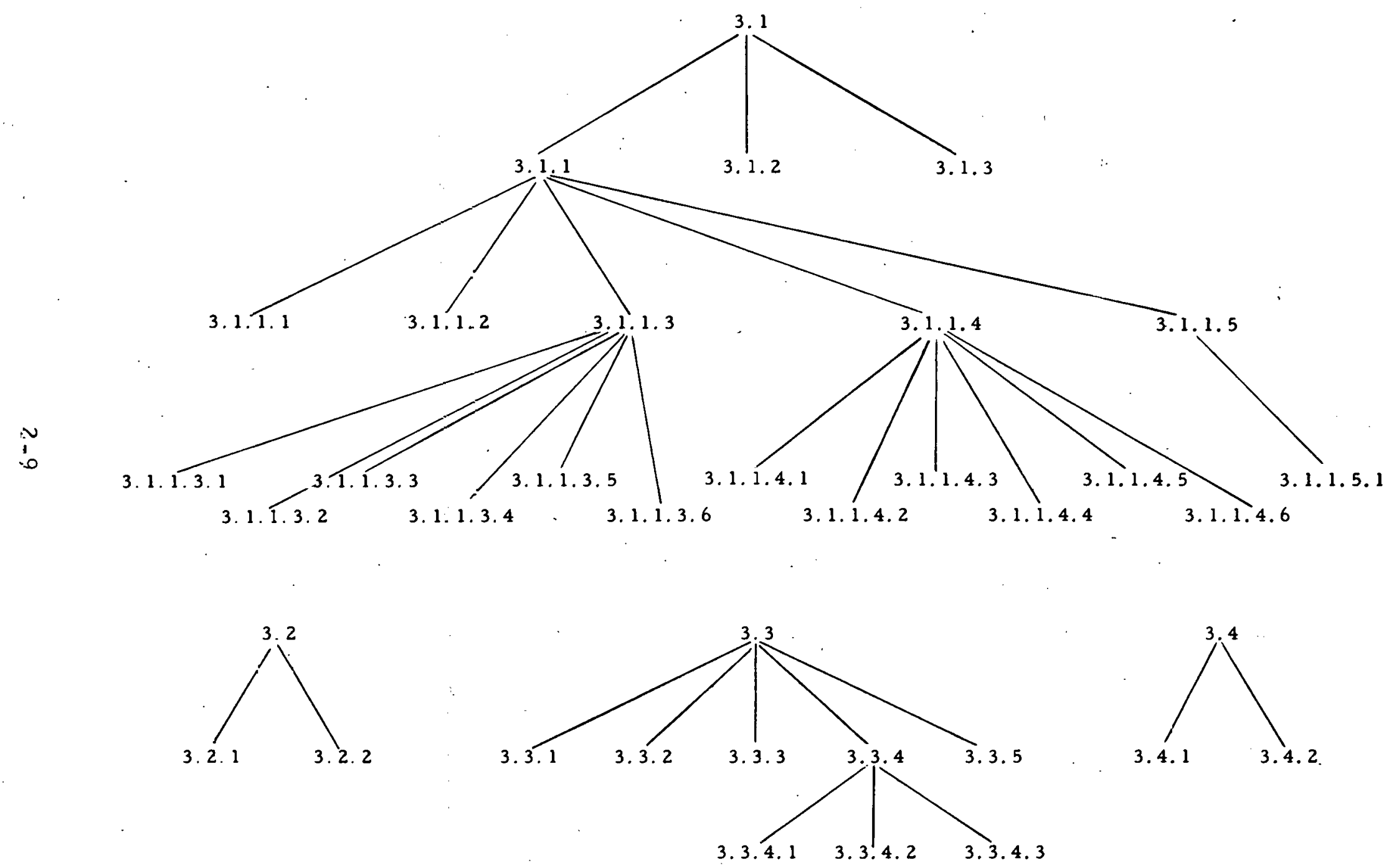

Figure 2-1. Geothermal EIDS Abbreviated Recipient Dimension Tree 
Table 2-4. Triads With No Impact Entries

$$
\begin{aligned}
& 1.3 .2-2.1-3.1 .1 .4 .3 \\
& \text { 3. } 1.1 .4 .5 \\
& 3.1 .1 .4 .6 \\
& 1.3 .2-2.2-3.1 .1 .3 .5 \\
& 3.1 .1 .4 .3 \\
& 3.1 .1 .4 .6 \\
& 3.3 .4 .3 \\
& 1.3 .2-2.3-3.1 .1 .1 \\
& 3.1 \cdot 1 \cdot 3.4 \\
& 3.1 .1 .3 .5 \\
& 3.1 .1 .3 .6 \\
& \text { 3. } 1.1 .4 \\
& \text { 3. } 1.1 .4 .1 \\
& 3.1 .1 .4 .2 \\
& 3.1 .1 .4 .3 \\
& 3.1 .1 .4 .4 \\
& 3.1 .1 .4 .5 \\
& 3.1 .1 .4 .6 \\
& 3.1 .1 .5 \\
& \text { 3. } 1.1 .5 .1 \\
& \text { 3. } 3.4 .3 \\
& 3.4 .2 \\
& 1.3 .2-2.4-3.1 .1 .1 \\
& \text { 3. } 1.1 .2 \\
& \text { 3.1.1.3 } \\
& \text { 3. } 1.1 .3 .1 \\
& \text { 3. 1.1.3.2 } \\
& \text { 3.1.1.3.3 } \\
& \text { 3. 1.1.3. } 4 \\
& \text { 3. 1.1.3.5 } \\
& 3.1 .1 .3 .6 \\
& 3.1 .1 .4 .3 \\
& 3.1 .1 .4 .5 \\
& \text { 3. } 1.1 .4 .6 \\
& 3.1 .1 .5 \\
& \text { 3. 1.1.5.1 } \\
& \text { 3. } 1.3 \\
& \text { 3. } 3.4 .3 \\
& \text { 3. } 3.5 \\
& 3.4 \\
& \begin{aligned}
1.3 .2-2.4- & 3.4 .1 \\
& 3.4 .2 \\
& \\
1.3 .2-2.5- & 3.1 .1 .3 .1 \\
& 3.1 .1 .3 .2 \\
& 3.1 .1 .3 .3 \\
& 3.1 .1 .3 .4 \\
& 3.1 .1 .3 .6 \\
& 3.1 .1 .4 .3 \\
& 3.1 .1 .4 .4 \\
& 3.1 .1 .4 .5 \\
& 3.1 .2 \\
& 3.1 .3
\end{aligned} \\
& 1.3 .2-2.6-3.1 .1 .1 \\
& \text { 3. } 1.1 .3 \\
& \text { 3.1.1.3.1 } \\
& \text { 3.1.1.3.2 } \\
& 3.1 .1 .3 .3 \\
& \text { 3. 1. 1. } 3.4 \\
& 3.1 .1 .3 .5 \\
& 3.1 .1 .3 .6 \\
& 3.1 .1 .4 \\
& \text { 3.1.1.4.1 } \\
& \text { 3.1.1.4.2 } \\
& \text { 3.1.1.4.3 } \\
& 3.1 .1 .4 .4 \\
& \text { 3.1.1. } 4.5 \\
& 3.1 .1 .4 .6 \\
& 3.1 .1 .5 \\
& \text { 3.1.1.5. } 1 \\
& 1.3 .2-2.7-3.1 .1 \\
& \text { 3.1.1. } 1 \\
& \text { 3. } 1.1 .2 \\
& \text { 3. } 1.1 .3 \\
& 3.1 .1 .3 .1 \\
& 3.1 .1 .3 .2 \\
& 3.1 .1 .3 .3 \\
& 3.1 .1 .3 .4 \\
& \text { 3. 1.1.3.5 } \\
& 3.1 .1 .3 .6
\end{aligned}
$$


Table 2-4. Triads With No Impact Entries (Continued)

$$
\begin{aligned}
& 1.3 .2-2.7-3.1 .1 .4 \\
& 3.1 .1 .4 .1 \\
& 3.1 .1 .4 .2 \\
& 3.1 .1 .4 .3 \\
& 3.1 .1 .4 .4 \\
& 3.1 .1 .4 .5 \\
& 3.1 .1 .4 .6 \\
& 3.1 .1 .5 \\
& \text { 3. } 1.1 .5 .1 \\
& 1.3 .2-2.8-3.1 .1 .3 .2 \\
& 3.1 \cdot 1 \cdot 3 \cdot 3 \\
& 3.1 .1 .3 .4 \\
& 3.1 .1 .3 .5 \\
& \text { 3. } 1.1 .3 .6 \\
& 3.1 .1 .4 .3 \\
& 3.1 .1 .4 .5 \\
& \text { 3.1.1.5 } \\
& 3.1 .1 .5 .1 \\
& 3.3 .4 .3 \\
& 1.3 .2-2.9-3.1 .1 .3 .2 \\
& 3.1 .1 .3 .3 \\
& \text { 3. } 1.1 .3 .4 \\
& 3.1 .1 .3 .5 \\
& \text { 3.1.1.4.3 } \\
& 3.1 .1 .5 .1
\end{aligned}
$$




\section{REFERENCES}

1. Energy Alternatives: A Comparative Analysis, The Science and Public Policy Program; University of Oklahoma, Norman, Oklahoma (May 1975).

2. ERDA Subject Indexing and Retrieval Thesaurus, TID-7000, U.S. Energy Research and Development Administration (June 1975).

3. Counties and County Equivalents of the States of the United States, Federal Information Processing Standards Publication $6-2$ (15 September 1973).

4. Ständard Metropolitan Statistical Areas, Federal Information Processing Standards Publication 8-4 (June 30, 1974). 
'b 


\section{APPENDIX A}

\section{ENVIRONMENTAL IMPACT DIRECTORY SYSTEM}

DIMENSION TABULATION 
1.

1. 1 .

1. 1.1

1.1.1. 1

1. 1.2

1.1.2. 1

1. 1.2 .2

1. 1.3

1. 1.4

1. 2

1. 2.1

1.2.1.1

1. 2. 1.2

1. 2.2

1. 3

1. 3.1

1. 3.2

1. 3.2 .1

1. 3. 2.1 .1

1.3.2.1.2

1.3.2.1.2. 1

1.3.2.1.2. 2

1.3.2.1.2. 3

1. 3.2 .1 .3

1.3 .2 .2

1. 3.2 .3

1. 3.2 .4

1. 3.3

1. 3.4

1. 4

1. 4.1

$1,4.2$

1. 4.3

1. 4.4

1. 4.5

1. 5

1. 5.1

1. 5.2

1. 5.3

1. 5.4

1. 5.5
Technolog $\dot{y}$

Fossil

Coal

: Direct Use*

Petroleum

Oil

Shale Oil

Natural Gas

Peat

Nuclear*

Fission

Non-breeder reactors*

Breeder reactors

Fusion*

Geopower

Hydroelectric power

Geothermal energy

Hydrothermal systems

Dry-steam systems

Hot-water systems

High temperature*

Moderate temperature*

Low temperature*

Geopressured systems

Hot-dry-rock systems

Normal-gradient systems*

Magma systems

Tidal power

Wind power

Solar Energy Conversion

Solar thermal power plants

Photovoltaic conversion

Ocean thermal gradient*

Biomass conversion*

Solar heating

Energy Conservation

Waste conversion*

Power transmission

Transportation

Waste heat utilization

Air conditioning 
1.6

1. 7

1. 7.1

1. 7.2

1. 7.3

1. 7.4

1. 7.5

1.7 .6

1. 7.7

1.8

1.9
Magnetohydrodynamic power*

Energy Storage

Pumped hydro*

Compressed air

Thermal*

Electrochemical*

Flywheels

Chemical*

Superconducting magnetic*

Thermoelectric power*

Thermionic power*

Each Technulugy las a final subheading on activity:
. A
. B
Exploration
Construction
. C
. D
. E
. F
. G
. $\mathrm{H}$
Exploitation
Transformation *
Transport
Waste Disposal/Waste Processing
Termination
Induced Technology

For example, 1.3.2. $\mathrm{C}$ is the exploitation of geothermal energy. 
2

2. 1

2. 1.1

2. 1.1 .1

2. 1.1 .2

2. 1.1 .3

2. 1.2

2. 2

2. 2.1

2. 2. 1.1

2.2.1.1. 1

2. 2. 1. 1.2

2. 2.1.1.2. 1

2. 2. 1. 1.2. 1.1

2. 2. 1.1.2.1.2

2. 2.1 .1 .2 .1 .3

2. 2. 1. 1. 2.1. 4

2.2.1.1.2.1.5

2. 2. 1. 1.2.1.6

2. 2. 1. 1.2. 2

2. 2.1.1.2.2.1

2. 2. 1. 1.2. 2.2

2. 2. 1. 1.2.2.3

2. 2. 1. 1.2.2. 4

2. 2.1.1.2.2.5

2.2.1.1.2.2.6

2. 2.1 .1 .2 .3

2. 2. 1. 1.2.3. 1

2. 2. 1. 1.2.3. 2

2. 2.1.1.2.3. 3

2.2.1.1.2.3. 4

2. 2.1.1.2.3. 5

2. 2. 1. 1.2. 4

2. 2. 1. 1. 2. 4.1

2.2.1.1.2. 4.2

2. 2.1.1.2. 4.3

2.2.1.1.2. 4.4

2. 2. 1.1.2. 4.5

2. 2.1.1.2. 5

2. 2.1.1.2. 5.1

2.2.1.1.2. 5.2

2.2.1.1.2.5.3

\author{
Residuals* \\ Earth residuals* \\ Land alteration * \\ Grading* \\ Excavation \\ Drilling \\ Solid wastes
}

Water residuals*

Chemical residuals*

Dissolved solids*

Total dissolved solids*

Trace elements*

Alkali metals (Group I)

Cesium

Francium

Lithium

Potassium

Rubidium

Sodium

Alkaline earth metals (Group II)

Barium

Beryllium

Calcium

Magnesium

Radium

Strontium

Group III elements*

Aluminum

Boron

Gallium

Indium

Thallium

Group IV elements*

Cárbon

Germanium

Lead

Silicon

$\mathrm{Tin}$

Group V elements *

Antimony

Arsenic

Bismuth

* Not in ERDA Thesaurus 
2. 2. 1.1.2. 5.4

2. 2. 1.1 .2 .6

2.2.1.1.2.6.1

2.2.1.1.2.6. 2

2. 2.1.1.2.6.3

2.2.1.1.2.6. 4

2. 2. 1. 1.2. 7

2. 2. 1.1.2.7. 1

2.2.1.1.2.7. 2

2. 2. 1.1.2.7. 3

2. 2. 1. 1.2.7. 4

2. 2. 1. 1.2.7. 5

2. 2.1 .1 .2 .8

2. 2. 1.1.2.8.1

2. 2. 1. 1.2.8. 2

2. 2. 1.1 .2 .8 .3

2. 2.1 .1 .2 .9

2. 2. 1.1.2.9. 1

2. 2.1.1.2.9. 2

2. 2. 1. 1.2. 9.3

2. 2.1.1.2.9. 4

2. 2.1.1.2.9. 5

2. 2.1.1.2.9.6

2.2.1.1.2.9. 7

2. 2.1 .1 .2 .9 .8

2.2.1.1.2.9.9

2. 2. 1. 1.2.9. 10

2.2.1.1.2.9.11

2.2.1.1.2.9.12

2. 2.1.1.2.9.13

2. 2.1.1.2.9. 14

2.2.1.1.2.9. 15

2. 2.1.1.2.9.16

2.2.1.1.2.9.17

2. 2. 1.1 .2 .9 .18

2.2.1.1.2.9.19

2.2.1.1.2.9.20

2. 2. 1.1.2.9.21

2. 2. 1. 1.2.9. 22

2. 2. 1.1.2.9. 23

2. 2.1.1.2.9.24

2.2.1.1.2. 9.25

2. 2. 1. 1.2. 9.26

* Not in ERDA Thesaurus
Phosphorus

Group VI elements*

Polonium

Selenium

Sulfur

Tellurium

Halogens

Astatine

Bromine

Chlorine

Fluorine

Iodine

Group IIB elements*

radmium

Mercury

Zinc

Transition elements

Chromium

Cobalt

Copper

Gold

Hafnium

Iridium

Iron

Manganese

Molybdenum

Nickel

Niobium

Osmiụm

Palladium

Platinum

Rhenium

Rhodium

Ruthenium

Scandium

Silver

Tantalum

Technetium

Titanium

Tungsten

Vanadium

Yttrium

Zirconium 
2. 2. 1. 1.2. 10

2. 2. 1.1 .2 .10 .1

2. 2. 1.1.2.10.2

2. 2.1.1.2.10.3

2. 2.1 .1 .2 .10 .4

2. 2.1.1.2.10.5

2. 2. 1. 1.2. 10.6

2. 2.1.1.2.10.7

2. 2.1.1.2. 10.8

2.2.1.1.2. 10.9

2. 2.1.1.2.10.10

2.2.1.1.2.10.11

2.2.1.1.2.10.12

2.2.1.1.2.10.13

2. 2.1.1.2.10.14

2.2.1.1.2.10.15

2.2.1.1.2.11

2.2.1.1.2.11.1

2. 2. 1. 1.2. 11.2

2. 2.1.1.2.11.3

2.2.1.1.2.11. 4

2.2.1.1.2.11.5

2.2.1.1.2.11.6

2. 2.1.1.2.11.7

2. 2.1 .1 .2 .11 .8

2.2.1.1.2.11.9

2.2.1.1.2.11.10

2.2.1.1.2.11.11

2.2.1.1.2.11.12

2. 2.1.1.2.11.13

2.2.1.1.2.11. 14

2. 2. 1.1.2.11.15

2. 2. 1. 1.2. 12

2.2.1.1.2.12. 1

2.2.1.1.2.12.1. 1

2.2.1.1.2.12.1.2

2. 2.1.1.2.12.1. 3

2. 2. 1. 1.2.12.1. 4

2. 2. 1.1.2.12.1.5

2. 2. 1.1.2.12.1.6

2.2.1.1.2.12.1.7

2. 2.1.1.2.13

2. 2. 1. 1.2.13.1

2. 2. 1.1.2.13.1.1
Rare earths

Cerium

Dysprosium

Erbium

Europium

Gadolinium

Holmium.

Lanthanum

Lutetium

Neodymium

Praseodymium

Promethium

Samarium

Terbium

Thulium

Ytterbium

Actinides

Actinium

Americium

Berkelium

Californium

Cürium

Einsteinium

Fermium

Lawrencium

Mendelevium

Neptunium

Nobelium

Plutonium

Protactinium

Thorium

Uranium

Inorganic compounds

Oxygen compounds

Borates

Carbonates

Hydroxides

Nitrates

Oxides

Phosphates

Sulfates

Organic compounds

Pesticides

Aldrin*

* Not in ERDA Thesaurus 
2.2.1.1.2.13.1.2

2. 2.1.1.2.13.1.3

2. 2. 1.1.2.13.1.4

2. 2. 1.1.2.13.1.5

2.2.1.1.2.13.1.6

2. 2.1.1.2.13.1.7

2.2.1.1.2.13.1.8

2.2.1.1.2.13.1.9

2.2.1.1.2.13.1.10

2. 2.1.1.2.13.1.11

2. 2.1.1.2.13.1.12

2.2.1.1.2.13.1.13

2. 2. 1. 1.2.13.1.14

2. 2.1.1.2.13.1.15

2. 2.1.1.2.13.1.16

2.2.1.1.2.13.1.17

2.2.1.1.2.13.2

2.2.1.1.2.13.3

2.2.1.1.2.13.4

2. 2.1 .1 .2 .13 .5

2. 2.1 .2

2. 2.1 .3

2. 2.1 .4

2. 2.1 .5

2. 2. 1.5 .1

2. 2. 1. 5.2

2. 2. 1.5 .3

2. 2.1. 5.4

2.2.1.5.4. 1

2. 2. 1. 5. 4.2

2. 2. 1.5 .4 .3

2. 2. 1.5 .4 .4

2. 2.1 .5 .4 .5

2. 2.1.5.4. 6

2.2.1.5. 5

2. 2.1 .6

2. 2.2

2. 2.2 .1

2. 2.2. 2

2. 2.2. 2.1

2. 2.3

2. 2. 3.1

2. 2. 3.2

2. 2.3 .3

2. 2. 3.4

* Not in ERDA Thesaurus
Dieldrin

Chlordane*

2, 4-D*

$2,4,5-T P *$

DDT

Demeton*

Endosulfan*

Endrin*

Guthion*

Heptachlor*

Lindane*

Malathion

Methoxychlor*

Mirex*

Parathion*

Toxaphene*

Phenols

Polychlorinated biphenyls (PCBs)

Oils and greases

Phthalates

Acidity/Alkalinity

Nutrients

Chemical oxygen demand*

Dis solved gases*

Oxygen

Ammonia

Hydrogen sulfide

Rare gases

Argon

Helium

Krypton

Neon

Radon

Xenon

Total dissolved gases*

Salinity

Biological residuals*

Biological oxygen demand*

Bacteria

Coliform bacteria

Solid residuals*

Suspended solids

Turbidity

Bed load

Asbestos 
2. 2.4

2. 2.4 .1

2. 2. 4.1 .1

2. 2.4 .1 .2

2. 2. 4.1 .3

2. 2.4 .2

2. 2. 4.2 .1

2. 2.4 .2 .2

2. 2. 4.2 .3

2. 2. 4.2 .4

2. 2. 4.2 .5

2. 2.5

2. 2.6

2. 2.6 .1

2. 2.6 .2

2. 2.7

2.2.7. 1

2. 2.7 .2

2. 3

2. 3.1

2. 3.1 .1

2. 3.1 .2

2. 3.1 .3

2. 3. 1.4

2. 3.1 .5

2. 3.1 .6

2. 3.1 .7

2. 3.1 .8

2. 3.1 .9

2. 3. 1.10

2. 3.2

2. 3.2 .1

2. 3.2 .2

2. 3.2 .3

2. 3.3

2. 3. 3.1

2. 3.3 .2

2. 3.4

2. 3.5

2. 3.6

2. 4

2. 4.1

2. 4.1.1

* Not in ERDa Thesaurus
Radioactive wastes

Activity levels Alpha particles

Beta particles

Gamma radiation

Specific isotopes*

Tritium

Strontium -90

Cesium-137

Radium -226

Radium-227

Thermal residuals*

Water use*

Consumption*

Volume/flow rate

Aesthetic qualities*

Taste*

Color

Atmospheric residuals *

Chemical residuals*

Sulfur oxides

Nitrogen oxides

Hydrocarbons

Photochemical pollutants *

Odor

Carbon monoxide

Carbon dioxide

Hydrogen sulfide

Hydrogen

Ammonia

Particle-Aerosol residuals *

Fly ash

Trace elements*

Asbestos

Radioactive wastes

Tritium

Radon

Thermal residuals*

Biological residuals*

Water vapor residuals*

Biological residuals*

Animal residuals*

Eliminated species* 
2. 4.1 .2

2. 4.1 .3

2. 4.2

2. 4.2 .1

2. 4.2 .2

2. 4.2 .3

2. 5

2. 5.1

2. 5.2

2. 5.3

2. 5.4

2,6

2. 6.1

2. 6.1 .1

2.6.1.2

2. 6.1 .3

2. 6.2

2. 6.3

2. 7

2. 7.1

2. 7.2

2. 7.3

2. 7.4

2. 7.5

2. 8

2. 8.1

2. 8.2

2. 8.3

2. 8.4

2. 9

2. 9.1

2. 9.2

2. 9.3

2. 9.4

2. 9.5

* Not in ERDA Thesaurus
Endangered species* Introduced species *

Plant residuals *

Eliminated species*

Endangered species*

Introduced species *

Social-Cultural residuals*,

Social residuals*

Cultural residuals* (e.g., elimination of an historic landmark)

Aesthetic residuals* (e.g. , elimination of a view)

Land use

Economic residuals*

Work force*

Employment rate

Skills mix

Wage i'ates

Housing supply *

Business activity*

Public health residuals*

Physiological residuals* (e.g., "black lung' disease)

Psychological residuals* (e.g., psychological effects of noise)

Air quality*

Water quality*

Noise

Infrastructure residuals *

Transportation systems

Communications

Energy supplies

Public services*

Catastrophic residuals*

Fires

Explosions

Blowouts

Meltdown

Earthquakes 
3.

3. 1

3. 1.1

3. 1. 1.1

3. 1.1 .2

3. 1.1 .3

3. 1.1 .3 .1

3. 1.1 .3 .2

3. 1. 1.3. 3

3. 1.1 .3 .4

3. 1.1 .3 .5

3. 1.1 .3 .6

3. 1.1 .4

3.1.1.4. 1

3. 1.1 .4 .2

3. 1.1 .4 .3

3. 1. 1. 4.4

3. 1. 1. 4.5

3. 1.1 .4 .6

3. 1.1 .5

3. 1.1 .5 .1

3. 1.1 .5 .2

3. 1.1 .5 .3

3. 1.2

3.1.2. 1

3. 1.2.1. 1

3. 1.2 .1 .2

3.1.2.1. 3

3.1.2.1. 4

3. 1.2. 2

3.1.2.2. 1

3. 1.2.2. 2

3.1.2.2. 3

3. 1.2 .3

3. 1.2.3.1

3. 1.2.3. 2

3. 1.3

3. 1.3 .1

3. 1.3 .2

3. 1.3 .2 .1

3. 1.3.2. 2

3. 1. 3.2. 3

* Not in ERDA Thesaurus

\section{Recipient*}

Physical and Chemical Domain* Earth*

Topography

Resources

Slow processes*

Weathe ring *

Solution*

Sorption/Complexing *

Deposition

Compaction*

Subsidence*

Rapid processes*

Flood

Erosion

Collapse*

Lands side / Ava lanche*

Seismicity*

Air movement*

Unique features*

Aquifer recharge

Aquicludes

Geothermal fields

Water

Seas

Estuaries

Coastal

Continental shelf

Deep ocean*

Surface waters

Lakes

Rivers

Snow and ice*

Ground water

Aquifers

Permafrost

Air

Air quality

Mic romete or ology*

Wind

Turbulence

Modification* 
3. 1.3 .3

3. 1.3.3.1

3.1.3.3.2

3. 1.3 .3 .3

3.1.3.3. 4

3. 1.3 .3 .5

3. 2

3. 2.1

3.2. 1.1

3.2. 1.2

3. 2.1 .3

3.2. 1.4

3.2. 1.5

3.2. 1.6

3. 2. 1.7

3. 2.1 .8

3. 2.1 .9

3. 2. 1.10

3. 2. 1.11

3. 2.1 .12

3. 2.1 .13

3. 2.1 .14

3. 2.1 .15

3. 2.1 .16

3. 1.2 .17

3. 2. 1.18

3. 2. 1.19

3. 2. 1.19 .1

3.2.1.19.2

3. 2. 1.19 .3

3. 2.1 .19 .4

3.2.1.19. 5

3.2.1.19.6

3.2.1.19.7

3. 2.1 .20

3.2.1.21

3. 2. 1.22

3. 2. 1.23

3. 2.1.23.1

3. 2. 1.23 .2

3.2.1.23. 3

3. 2. 1.23 .4

3.2.1.23. 5
Climatology*

Atmospheric precipitations

Wind

Temperature*

Cloud cover.

Insolation

\section{Biological domain*}

Animals

Protozoa

Mesozoa*

Porifera* (e.g., sponges)

Coelenterata (Cnidaria) (e.g. , jellyfish, sea anemones, coral)

Ctenophora*

Fiatyhelminthes (e.g., flatworms)

Rhynchocoela* (Nemertina)

Rotifera*

Gastrotricha*

Kinorhyncha* (Echinoderida)

Priapulida*

Gordiacea* (Nematomorpha) (e.g., worms)

Nematoda (e.g., eelworms)

Acanthocephala* (e.g., intestinal worms)

Entoprocta* (Calyssozoa)

Bryozoa* (Polyzoa)

Phoronida*

Brachiopoda *

Mollusca

Polyplacophora* (e.g., chitons)

Aplacophora*

Monoplacophora*

Gastropoda* (e.g., snails)

Scaphopoda* (e.g., tusk shells)

Bivalvia (e.g., clams)

Cephalopoda* (e.g., octopus, squids)

Spinucula*

Echiura*

Annelida (e.g., earthworms, leeches)

Arthropoda

Onychophora*

Pauropoda*

Diplopoda* (e.g., millipedes)

Chilopoda* (e.g., centipedes)

Symphyla* (e.g., garden "centipede")

* Not in ERDA The saurus 
3.2 .1 .23 .6

3. 2.1 .23 .7

3. 2. 1.23 .8

3.2. 1.23 .9

3.2. 1.23 .10

3.2.1.23.11

3. 2. 1.23 .12

3. 2.1 .24

3. 2.1 .25

3. 2.1 .26

3. 2. 1.26. 1

3.2 .1 .26 .2

3. 2. 1.26 .3

3. 2.1 .26 .4

3. 2.1 .26 .5

3. 2.1 .27

3. 2.1 .28

3. 2. 1.28 .1

3.2.1.28.2

3. 2.1 .28 .3

3. 2.1 .28 .4

3. 2. 1.28 .5

3. 2.1 .28 .6

3. 2. 1. 28.7

3. 2.1 .28 .8

3.2 .1 .28 .9

3.2. 1.28 .10

3.2.1.28. 11

3. 2.1 .28 .12

3.2.1.29

3. 2.1 .30

3.2.1.30.1

3.2.1.30.2

3.2. 1.30 .3

3. 2.2

3. 2. 2.1

3. 2.2 .2

3. 2.2 .3

3. 2. 2.4

3. 2.2 .5

3. 2.2 .6
Insecta (e.g., beetles, wasps, moths, flies, lice)

Crustacea (e.g., crabs, shrimp, barnacles, sow bugs)

Merostomata*

Arachnida (e.g., spiders, scorpions, ticks)

Pycnogonida*

Pentastomida*

Tardigrada* (e.g., "water bears")

Chaetognatha* (e.g., arrowworms)

Pogonophora*

Echinode rmata

Crinoidea* (e.g., sea lilies, feather stars)

Holothuroidea* (e.g., sea cucumbers)

Echinoidea* (e.g., sea urchins)

Asteroidea* (e.g., starfish)

Ophiuroidea* (e.g., brittle stars)

Hemichordata*

Chordata $*$

Ascidiacea*

Thaliacea*

Larvacea*

Agnatha* (e.g., lampreys)

Chondrichthyes (e.g., sharks)

Osteichthyes* (e.g., fish)

Crossopterygii* (e.g., coelacanths)

Dipnoi* (lungfish)

Amphibia (e.g., frogs, toads, salamanders)

Reptilia (e.g., turtles, lizards, snakes)

Aves* (e.g. , birds)

Mammalia.

Endangered species*

Migration*

Corridors*

Barriers*

Vectors

Plants

Protophyta*

Cyanophyta* (e.g., blue-green algae)

Chrysophyta*

Chlorophyta* (e.g., green algae)

Euglenophyta *

Pyrrophyta*

* Not in ERDA Thesaurus 
3.2 .2 .7

3.2.2. 8

3. 2.2 .9

3.2. 2.10

3.2. 2.11

3.2.2. 12

3. 2. 2.13

3. 2. 2.14

3.2.2. 15

3.2.2. 16

3.2.2.17

3.2.2.17:1

3.2.2.17.1.1

3.2.2.17.1.2

3.2 .2 .17 .1 .3

3.2 .2 .17 .1 .4

3.2 .2 .17 .1 .5

3.2 .2 .17 .1 .6

3.2 .2 .17 .1 .7

3.2.2.17.2

3. 2.2.17.2.1

3.2.2.17.2. 2

3.2 .2 .17 .2 .3

3.2 .2 .17 .2 .4

3.2. 2.18

3. 2. 2.19

3.2.2.19.1

3. 2.2. 19.2

3.2.2. 19.3

* Not in ERDA Thesaurus
Cryptophyta *

Phaeophyta * (e.g., brown algae)

Rhodophyta* (e.g., red algae)

Mycota* (e.g., fungi, bacteria)

Bryophyta* (e.g., mosses)

Psilotophyta*

Lycopodiophyta* (e.g., scale trees)

Equisetophyta* (e.g., horsetail)

Pteridophyta* (Filices) (e.g., ferns)

Pinophyta* (Gymnospermae) (e.g. , conifers, ginkoes)

Magnoliophyta* ngiospermae) Magnoliopsida* (Dicotyledoneae)

Magnoliales* (e.g., magnolias, laurels, peppers, water lilies)

Ranunculidae (e.g., peonies, buttercups, poppies)

Hamamelididae* (e.g., sweet gum, nettles, beeches, chestnuts, walnuts)

Caryophyllidae* (e.g., carnations, buckwheat)

Dilleniidae* (e.g., camellias, violets, melons, willows, azaleas, primulas)

Rosidae* (e.g., roses, legumes, myrtles, citrus, maples: flax, grapes, ash)

Asteridae* (e.g. , snapdragons, asters, daises, thistles, verbenas)

Liliopsida* (Monocotyledonae)

Alismidae* (e.g., pond weeds)

Liliidae* (e.g., lilies, iris, bananas, orchids)

Commelinidae* (e.g., rushes, sedges, bromeliads)

Arecidae* (e.g., palms, arums, cattails)

Endangered species

Migration*

Corridors*

Barriers*

Vectors 
3.3

3. 3.1

3. 3.1 .1

3. 3.1 .2

3. 3.1 .3

3. 3.1 .4

3. 3.1 .5

3. 3.1 .6

3. 3.1 .7

3. 3.1 .8

3. 3.1 .9

3. 3.1 .10

3. 3.1 .11

3. 3.2

3. 3.2 .1

3. 3.2 .2

3. 3.2 .3

3. 3.2 .4

3. 3.2. 5

3. 3.2 .6

3. 3.2 .7

3. 3.2 .8

3. 3.3

3. 3.3 .1

3. 3.3 .2

3. 3. 3. 2.1

3. 3. 3.2. 2

3. 3.3 .3

3. 3.3 .4

3. 3.3 .5

3. 3.3 .6

3. 3.4

3. 3.4 .1

3. 3.4 .2

3. $3,4,3$

3. 3. 4.3 .1

3. 3.4 .3 .2

3. 3.4 .3 .3

3. 3.5

3. 3. 5.1

3. 3. 5.2

3. 3.5 .3

3. 3.5 .4

3. 3.5 .5

3. 3.5 .6

* Not in ERDa Thesaurus
Social-Cultural Domain*

Land use

Wilderness

Wetlands*

Forests

Grazing lands*

Agricultural lands*

Residential lands*

Commercial lands*

Industrial lands*

Resource recovery areas*

Deserts

Public lands

Recreation*

Fishing*

Swimming*

Boating*

Hunting *

Camping/Hiking *

Picnicking

Winter sports*

Resorts*

Aesthetics*

Scenic views*

Open space*

Wilderness*

Parks and preserves*

Landscape*

Archeological/Historical sites*

Unique physical characteristics*

Unique ecological characteristics*

Quality of life*

Health and safety*

Life style*

Population characteristics*

Age distribution*

Education

Ethnic and racial characteristics

Legal aspects

Legislation

Regulations

Insurance

Real estate

Licenses

Patents 

3. 4 .
3. 4.1
3. 4.1 .1
3. 4. 1.1.1
3. 4.1 .1 .2
3. 4.1 .1 .3
3. 4.1 .2
3. 4.1 .3
3. 4.1 .4
3. 4.1 .4 .1
3. 4.1 .4 .2
3. 4.1 .4 .3
3. 4.1 .5
3. 4.2
3. 4.2 .1
3. 4.2 .2
3. 4.2 .3
3. 4. 2.4
3. 4.2 .5
3. 4.2 .5 .1
3. 4.2.5. 2
3. 4.2 .6
3. 4.2 .6 .1

Economic domain*

Demographic factors*

Work force*

Employment rate*

Skills mix*

Wage rates*

Personal income*

Consumption and growth*

Public sector*

Revenues

Expenditures

Debt

Private sector*

Infrastructure domain *

Transportation systems

Energy supplies

Waste disposal (including pollution control)*

Communications

Structureg*

Public*

Private*

Public services*

Educational system*

* Not in ERDA Thesaurus 
I

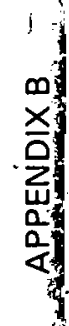

?

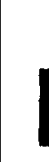


APPENDIX B

1

GEOTHERMAL ENVIRONMENTAL IMPACT DIRECTORY SYSTEM IMPACT CHECKLIST 


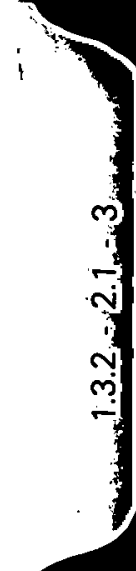

b 
Technology: $\quad 1.3 .2$

Geopower.

Geothermal Energy

Residual: $\quad 2.1$

Earth residuals

Recipient: $\quad 3.1$

Physical and chemical domain

Potential Environmental Impact: A. Exploration SEVERE

B. Construction SEVERE

C. Exploitation SEVERE

D. Transformation MINOR

E. Transport MODERATE

F. Waste disposal/processing SEVERE

G. Termination MODERATE

H. Induced technology UNASSESSED

Example Impacts: 1. C. Drilling, Blowout

2. A. Grading, Loss of Soil Resources

3. C. Drilling, Use of Water in Drilling Lubricant

Data Available: 1. GRID

2. USGS TOPOMAPS

3. ENDEX

4. NAW DEX

5. AEROS

6. IVEP

7. STORET

8. SWIRS

9. USGS GEOMAPS

Models Available: 1. SUWQI

2. PERCOL 
Technology: 1.3 .2

Geopower

\section{Geothermal Energy}

Residual: $\quad 2.1$

Earth residuals

Recipient: $\quad 3.1 .1$

Physical and chemical domain

Earth

Potential Environmental Impact: A. Exploration SEVERE

B. Construction SEVERE

C. Exploitation SEVERE

D. Transformation MINOR

E. Transport MODERATE

F. Waste disposal/processing SEVERE

G. Termination MODERATE

H. Induced technology UNASSESSED

Example Impacts: 1. A. Grading, Alteration of Topography

2. A. Grading, Erosion

3. A. Grading, Landslide

Data Available: 1. USGS TOPOMAPS

2. IVEP

3. SWIRS

4. USGS GEOMAPS

5. GRID

Models Available: 1. SUWQI

2. PERCOL 
Technology: $\quad 1.3 .2$

Geopower

Geothermal Energy

Residual: $\quad 2.1$

Earth residuals

Recipient: $\quad 3.1 .1 .1$

Physical and chemical domain

Earth

Topography

Potential Environmental Impact: A. Exploration MODERATE

B. Construction SEVERE

C. Exploitation MODERATE

D. Transformation NONE

E. Transport MINOR

F. Waste disposal/processing MINOR

G. Termination MINOR

$H$. Induced technology UNASSESSED

Example Impacts: 1. A. Grading, Road Building

2. B. Excavating, Building Sites

3. B. Grading, Holding Pond Construction

Data Available:

USGS TOPOMAPS

Models Available: 
Technology: $\quad 1.3 .2$

Geopower

Geothermal Energy

Residual: $\quad 2.1$

Earth residuals

Recipient: $\quad 3.1 .1 .2$

Physical and chemical domain

Earth

Resources

Potential Environmental Impact: A. Exploration MODERATE

B. Construction SEVERE

C. Exploitation SEVERE

D. Transformation MINOR

E. Transport MODERATE

F. Waste disposal/processing MINOR

G. Termination MODERATE

H. Induced technology UNASSESSED

Example Impacts: 1. A. Grading, Top Soil Destruction

2. E. Transport, Consumption of Fossil Fuel

3. C. Depletion of Heat Resources

Data Available: USGS GEOMAPS

Models Available: 
Technology: $\quad 1.3 .2$

Geopower

Geothermal Energy

Residual: $\quad 2.1$

Earth residuals

Recipient: $\quad 3.1 .1 .3$

Physical and chemical domain

Earth

Slow Processes

Potential Environmental Impact: A. Exploration MODERATE

B. Construction SEVERE

C. Exploitation SEVERE

D. Transformation NONE

E. Transport MINOR

F. Waste disposal/processing SEVERE

G. Termination MINOR

$\mathrm{H}$. Induced technology UNASSESSED

Example Impacts: 1. A. Grading, Promotion of Weathering

2. B. Excavation, Landfill Deposition

3. B. Grading, Land Fill Compaction

Data Available: 1. SWIRS

2. IVEP

Models Available: PERCOL 
Technology: $\quad 1.3 .2$

Geopower

Geothermal Energy

Residual: $\quad 2.1$

Earth residuals

Recipient: $\quad 3.1 .1 .3 .1$

Physical and chemical domain

Earth

Slow Processes

Weathering

Potential Environmental Impacis: A. Exploration MINOR

B. Construction SEVERE

C. Exploitation MINOR

D. Transformation NONE

E. Transport NONE

F. Waste disposal/processing NONE

G. Termination MINOR

H. Induced technology UNASSESSED

Example Impacts: 1. A, B. Weathering of Rock Exposed in Grading

Data Available:

Models Available:

B -6 
Technology: 1.3 .2

Geopower

Geothermal Energy

Residual: $\quad 2.1$

Earth Residuals

Recipient: $\quad 3.1 .1 .3 .2$

Physical and Chemical Domain

Earth

Slow Processes

Solution

Potential Environmental Impact:
A. Exploration
MINOR
B. Construction
MODERATE
C. Exploitation
MODERATE
D. Transformation
NONE
E. Transport
NONE
F. Waste disposal/processing SEVERE
G. Termination
MINOR
$\mathrm{H}$. Induced technology UNASSESSED

Example Impacts: 1. A, B, C,G. Leaching of Solubles from Exposed Soils.

2. F. Leaching of Brine Residues into Soils

Data Available:

Models Available: 
Technology: $\quad 1.3 .2$

Geopower

Geothermal Energy

Residual: 2.1

Earth Residuals

Recipient: 3.1.1.3.3

Physical and Chemical Domain

Earth

Slow Processes

Sorption/Complexing

Potential Environmental Impact: A. Exploration NONE

B. Construction NONE

C. Exploitation NONE

D. Transformation NONE

E. Transport NONE

F. Waste disposal/processing SEVERE

G. Termination NONE

H. Induced technology. UNASSESSED

Example Impacts: F. Sorption of Trace Metals by Clay Pond Liners

Data Available:

Models Available: PERCOL 
Technology: 1.3 .2

Geopower

Geothermal Energy

Residual: 2.1

Earth Residuals

Recipient: $3.1 \cdot 1 \cdot 3.4$

Physical and Chemical Domain

Earth

Slow Processes

Deposition

Potential Environmental Impact: A. Exploration MODERATE

B. Construction SEVERE

C. Exploitation MODERATE

D. Transformation NONE

E. Transport NONE

F. Waste disposal/processing MINOR

G. Termination MINOR

$H$. Induced technology UNASSESSED

Example Impacts: A, B, C. Stream Channel Filling by Deposition from Construction Activity

Data Available: SWIRS

Models Available: 
Technology: $\quad 1.3 .2$

Geopower

Geothermal Energy

Residual: ' 2.1

Earth Residuals

Recipient: 3.1 .1 .3 .5

Physical and Chemical Domain

Earth

Slow Processes

Compaction

Potential Environmental Impact: A. Exploration MINOR

B. Construction MODERATE

C. Exploitation NONE

D. Transformation NONE

E. Transport NONE

F. Waste disposal/processing NONE

G. Termination MINOR

H. Induced technology UNASSESSED

Example Impacts: 1. A, B. Compaction of Soil by Heavy Equipment

2. B. Compaction of Deposited Sediment from Construction Activity

3. G. Compaction of Fill in Restoring Original Contours

Data Available:

Models Available: 
Technology: $\quad 1.3 .2$

Geopower

Geothermal Energy

Residual: 2.1

Earth Residuals

Recipient: 3.1 .1 .3 .6

Physical and Chemical Domain

Earth

Slow Processes

Subsidence

Potential Environmental Impact: A. Exploration NONE

B. Construction NONE

C. Exploitation SEVERE

D. Transformation NONE

E. Transport NONE

F. Waste disposal/processing NONE

G. Termination NONE

H. Induced technology UNASSESSED

Example Impacts: C. Subsidence from Prolonged Removal of Geothermal Fluids

Data Available: IVEP

Models Available: 
Technology: 1.3 .2

Geopower

Geothermal Energy

Residual: 2.1

Earth Residuals

Recipient: 3.1.1.4

Physical and Chemical Domain

Earth

Rapid Processes

Potential Environmental Impact: A. Exploration SEVERE

B. Construction SEVERE

C. Exploitation SEVERE

D. Transformation NONE

E. Transport MODERATE

F. Waste disposal/processing SEVERE

G. Termination MODERATE

$\mathrm{H}$. Induced technology UNASSESSED

Example Impacts: 1. A, F. Drilling Blowout

2. B. Grading, Inducement of Landslide

3. B, C, G. Erosion Prior to Revegetation

Data Available: . 1. IVEP

2. USGS GEOMAPS

Models Available: 
Technology: $\quad 1.3 .2$

Geopower

Geothermal Energy.

Residual: 2.1

Earth Residuals

Recipient: 3.1 .1 .4 .1

Physical and Chemical Domain

Earth

Rapid Processes

Flood

Potential Environmental Impac's: A. Exploration MINOR

B. Construction SEVERE

C. Exploitation NONE'

D. Transformation NONE

E. Transport NONE.

F. Waste disposal/processing ' NONE

G. Termination MINOR

$H$. Induced technology UNASSESSED

Example Impacts: A, B, G. High Runoff after Denudation for Grading May Result in Flooding

Data Available:

Models Available: 
Technology: $\quad 1.3 .2$

Geopower

Geothermal Energy

Residual: 2.1

Earth Residuals

Recipient: 3.1 .1 .4 .2

Physical and Chemical Domain

Earth

Rapid Processes

Erosion

Potential Environmental Impact: A. Exploration MINOR

B. Construction SEVERE

C. Exploitation MODERATE

D. Transformation NONE

E. Transport MINOR

F. Waste disposal/processing MINOR

G. Termination MODERATE

H. Induced technology UNASSESSED

Example Impacts: A, B, C, E, F, G. High Runoff from Graded and Excavated Areas

Data Available:

Models Available: 
Technology: $\quad 1.3 .2$

Geopower

Geothermal Energy

Residual: 2.1.

Earth Residuals

Recipient: 3.1 .1 .4 .4

Physical and Chemical. Domain.

Earth

Rapid Processes

Lands lide/Avalanche

Potential Environmental Impací: A. Exploration MINOR

B. Construction SEVERE

C. Exploitation MINOR

D. Transformation NONE

E. Transport MINOR

F. Waste disposal/processing MINOR

G. Termination MINOR

H. Induced technology NONE

Example Impacts: 1. A, B, C, E, F, G. Landslide Triggered by Earth Moving

2. A, B. Avalanche Triggered by Blasting

Data Available:

Models Available: 
Technology: $\quad 1.3 .2$

Geopower

Geothermal Energy

Residual: 2.1

Earth Residuals

Recipient: . 3.1.1.5

Physical and Chemical Domain

Earth

Unique Features

Potential Environmental Impaci: A. Exploration MINOR

B. Construction NONE

C. Exploitation SEVERE

D. Transformation NONE

E. Transport NONE

F. Waste disposal/processing SEVERE

G. Termination NONE

$\mathrm{H}$. Induced technology NONE.

Example Impacts: 1. A. Contamination of Aquifer

2. C. Cessation of Surface Thermal Activity

(Hot Springs, Geysers)

Data Available:

Models Available: 
Technology: $\quad 1.3 .2$

Geopower

Geothermal Energy

Residual: 2.1

Earth Residuals

Recipient: 3.1 .1 .5 .1

Physical and Chemical Domain

Earth

Unique Features

Aquifer Recharge

Potential Environmental Impact: A. Exploration NONE

B. Construction NONE

C. Exploitation SEVERE

D. Transformation NONE

E. Transport NONE

F. Waste disposal/processing SEVERE

G. Termination NONE

$H$. Induced technology NONE

Example Impacts: F. Premature Cooling of Reservoir by Reinjection

Data Available:

Models Available: 
Technology: $\quad 1.3 .2$

Geopower

Geothermal Energy

Residual: 2.1

Earth Residuals

Recipient: 3.1 .2

Physical and Chemical Domain

Water

Potential Environmental Impact: A. Exploration SEVERE

B. Construction SEVERE

C. Exploitation MODERATE

D. Transformation NONE

E. Transport NONE

F. Waste disposal/processing SEVERE

G. Termination MINOR

H. Induced technology UNASSESSED

Example Impacts: 1. A, B, C, F. Contamination of Water from Drilling Mud Sumps

2. A, B, C. Contamination of Water by Human Activity

Data Available: 1. GRID

2. NAWDEX

3. STORET

Models Available: SUWQI 
Technology: $\quad 1.3 .2$

Geopower

Geothermal Energy

Residual: 2.1

Earth Residuals

Recipient: 3.1 .3

Physical and Chemical Domain

Air

Potential Environmental Impact: A. Exploration MINOR

B. Construction MODERATE

C. Exploitation MODERATE

D. Transformation NONE

E. Transport NONE

F. Waste disposal/processing NONE

G. Termination NONE

$\mathrm{H}$. Induced technology NONE

Example Impacts: 1. A, B, C. Dust Generated During Grading

2. A, B, C. Air Pollution Resulting from Vehicles and Machinery

Data Available: AEROS

Models Available: 
Technology: $\quad 1.3 .2$

Geopower

Geothermal Energy

Residual: 2.1

Earth Residuals

Recipient: 3.2

Biological Domain

Potential Environmental Impact: A. Exploration MODERATE

B. Construction SEVERE

C. Exploitation MODERATE

D. Transformation NONE

E. Transport MINOR

F. Waste disposal/processing SEVERE

G. Termination SEVERE

H. Induced technology UNASSESSED

Example Impacts: 1. A, B, C. Intrusion into Ecological Domain Drives away Animals

2. A, B, C. All Phases of Construction Damage Vegetation

Data Available:

Models Available: 
$\begin{aligned} \text { Technology: } & 1.3 .2 \\ \text { Geopower } & \text { Geothermal Energy }\end{aligned}$
Residual: 2.1
Earth Residuals
Recipient: 3.2 .1
Biological Domain
Animals

Potential Environmental Impact: A. Exploration 'MODERATE

B. Construction SEVERE

C. Exploitation MODERATE

D. Transformation NONE

E. Transport MINOR

F. Waste disposal/processing MODERATE

G. Termination SEVERE

$\mathrm{H}$. Induced technology UNASSESSED

Example Impacts: 1. A, B, C, E, G. Disturbance of Nesting and Feeding Areas

2. F. Waste Fluids Toxic to Some Species

Data Available:

Models Available: 
Technology: 1.3 .2

Geopower

Geothermal Energy

Residual: 2.1

Earth Residuals

Recipient: 3.2 .2

Biological Domain

Plants

Potential Environmental Impact: A. Exploration MINOR

B. Construction SEVERE

C. Exploitation MODERATE

D. Transformation NONE

E. Transport MINOR

F. Waste disposal/processing SEVERE

G. Termination MINOR

H. Induced technology UNASSESSED

Example Impacts: 1. A. Local Disturbance of Growth at Drilling Site and Access Roads

2. B, C, E, G. Removal of Growth During Grading

Data Available:

3. F. Waste Brines.Toxic to Growth

Models Available: 
Technology: $\quad 1.3 .2$

Geopower

Geothermal Energy

Residual: 2.1

Earth Residuals

Recipient: ' 3.3

Social-Cultural Domain

Potential Environmental Impact: A. Exploration

SEVERE

B. Construction

SEVERE

C. Exploitation

SEVERE

D. Transformation

MODERATE

E. Transport

SEVERE

F. Waste disposal/processing

SEVERE

G. Termination

SEVERE

H. Induced technology

UNASSESSED

Example Impacts: 1. B, E. Normal land use interrupted during construction pad and road grading.

2. B, E. Vehicular accidents a safety hazard

Data Available: 1: GRID

2. SWIRS

Models Available: 
Technology: $\quad 1.3 .2$

Geopower

\section{Geothermal Energy}

Residual: 2.1

Earth Residuals

Recipient: 3.3 .1

Social-Cultural Domain

Land Use

Potential Environmental Impact: A. Exploration MINOR

B. Construction SEVERE

C. Exploitation NONE.

D. Transformation NONE

E. Transport MINOR

F. Waste disposal/processing SEVERE

G. Termination SEVERE

H. Induced technology UNASSESSED

Example Impacts: 1. A. Temporary Dis ruption of Normal Use During Exploratory Operations

2. B, E. Barring of Access and Use During Grading

3. F. Waste Disposal Alters Land Productivity

4. G. Reestablishment of Land Use

Data Available: SWIRS

Models Available: 
Technology: 1.3 .2

Geopower

Geothermal Energy

Residual: 2.1

Earth Residuals

Recipient: 3.3 .2

Social-Cultural Domain

Recreation.

Potential Environmental Impact: A. Exploration MINOR

B. Construction SEVERE

C. Exploitation NONE

D. Transformation NONE

E. Transport MINOR

F. Waste disposal/processing SEVERE

G. Termination SEVERE

$\mathrm{H}$. Induced technology UNASSESSED

Example Impacts: A, B, E, F, G. Temporary Disruption of Recreational Activity During Grading and Drilling

Data Available:

Models Available: 
Technology: $\quad 1.3 .2$

Geopower

Geothermal Energy

Residual: 2.1

Earth Residuals

Recipient: 3.3 .3

Social-Cultural Domain

Aes thetics

Potential Environmental Impact: A. Exploration MINOR

B. Construction SEVERE

C. Exploitation SEVERE

D. Transformation MODERATE

E. Transport MODERATE

F. Waste disposal/processing MODERATE

G. Termination SEVERE

$H$. Induced technology UNASSESSED

Example Impacts: 1. A. Temporary Scarring of Land During Exploration

2. B, C, D, E, F. Grading Scars from Drilling Sites and Roads

3. G. Temporary Scars from Regrading to Original Contours

Data Available:

Models Available: 
Technology: 1.3 .2

Geopower

Geothermal Energy

Residual: 2.1

Earth Residuals

Recipient: 3.3 .4

Social-Cultural Domain

Quality of Life

Potential Environmental Impact: A. Exploration

MODERATE

B. Construction

SEVERE

C. Exploitation

MODERATE

D. Transformation

NONE

E. Transport

MODERATE

F. Waste disposal/processing

SEVERE

G. Termination

SEVERE

$\mathrm{H}$. Induced technology

UNASSESSED

Example Impacts: A, B, C, G. Hiring of labor from local Indian or SpanishAmerican populations may change life style.

Data Available:

Models Available: 
Technology: $\quad 1.3 .2$

Geopower

Geothermal Energy

Residual: 2.1

Earth Residuals

Recipient: 3.3.4.1

Social-Cultural Domain

Quality of Life

Health and Safety

Potential Environmental Impact:

A. Exploration MODERATE

B. Construction SEVERE

C. Exploitation MODERATE

D. Transformation NONE

E. Transport MODERATE

F. Waste disposal/processing SEVERE

G. Termination SEVERE

H. Induced technology UNASSESSED

Example Impacts: 1. A, C, F. Drilling Blowout

2. B,E. Vehicular Accidents

Data Available:

Models Available 
Technology: $\quad 1.3 .2$

Geopower

Geothermal Energy

Residual: 2.1

Earth Residuals

Recipient: 3.3 .4 .2

Social-Cultural Domain

Quality of Life

Life Style

Potential Environmental Impač: A. Exploration MINOR

B. Construction MODERATE

C. Exploitation MODERATE

D. Transformation NONE

E. Transport NONE

F. Waste disposal/processing NONE

G. Termination SEVERE

$\mathrm{H}$. Induced technology UNASSESSED

Example Impacts: A, B, C, G. Exploitation of GT Fields in Vicinity of Indian or Spanish-American Populations

Data Available:

Models Available: 
Technology: $\quad 1.3 .2$

Geopower

Geothermal Energy

Residual: 2.1

Earth Residuals

Recipient: 3.3 .4 .3

Social-Cultural Domain

Quality of Life

Population Characteristics

Potential Environmental Impacé: A. Exploration MINOR

B. Construction MODERATE

C. Exploitation MODERATE

D. Transformation NONE

E. Transport NONE

F. Waste disposal/processing NONE

G. Termination MODERATE

$\mathrm{H}$. Induced technology UNASSESSED

Example Impacts: A, B, C, G. Exploitation of GT Fields in Vicinity of Indian or Spanish-American Populations

Data Available:

Models Available: 
Technology: $\quad 1.3 .2$

Geopower

Geothermal Energy

Residual: $\quad 2.1$

Earth Residuals

Recipient: 3.3 .5

Social-Cultural Domain

Legal Aspects

Potential Environmental Impac^: A. Exploration SEVERE

B. Construction SEVERE

C. Exploitation SEVERE

D. Transformation NONE

E. Transport SEVERE

F: Waste disposal/processing SEVERE

G. Termination SEVERE

$\mathrm{H}$. Induced technology UNASSESSED

Example Impacts: A, B, C, E, F, G. Licenses, Permits, Insurance, Compliance with Environmental Requirements, Utility Regulations, Labor Laws

Data Available: GRID

Models Available: 
Technology: 1.3 .2

Geopower

Geothermal Energy

Residual: 2.1

Earth Residuals

Recipient: 3.4

Economic Domain

Potential Environmental Impacé: A. Exploration MINOR

B. Construction MODERATE

C. Exploitation SEVERE

D. Transformation MINOR

E. Transport SEVERE

F. Waste disposal/processing MODERATE

G. Termination MODERATE

$\mathrm{H}$. Induced technology UNASSESSED

Example Impacts: 1. C. Construction Camps Burden Local Telephone System

2. E. Heavy Equipment Disrupts Traffic Patterns

Data Available: 1. GRID

2. SWIRS

Models Available: 
Technology: $\quad 1.3 .2$

Geopower

Geothermal Energy

Residual: 2.1

Earth Residuals.

Recipient: 3.4 .1

Economic Domain

Demographic Factors

Potential Environmental Impact: A. Exploration MINOR

B. Construction MINOR

C. Exploitation MINOR

D. Transformation NONE

E. Transport MINOR

F. Waste disposal/processing MINOR

G. Termination MINOR

H. Induced technology UNASSESSED

Example Impacts: 1. A, B, C, E. Local Employment Affects Local Wage Scale

2. G. Termination has Negative Effect on Growth

Data Available: GRID

Models Available: 
Technology: 1.3 .2

Geopower

Geothermal Energy

Residual: 2.1

Earth Residuals

Recipient: 3.4 .2

Economic Domain

Infrastructure Domain

Potential Environmental Impaci: A. Exploration MINOR

B. Construction MODERATE

C. Exploitation SEVERE

D. Transformation MINOR

E. Transport SEVERE

F. Waste disposal/processing MODERATE

G. Termination MODERATE

$H$. Induced technology UNASSESSED

Example Impacts: 1. A, B, C, E. Construction Equipment Consumes Fuel

2. E. Heavy Equipment Burdens Local Road System

Data Available: SWIRS

Models Available: 

Technology: $\quad 1.3 .2$

Geopower

Geothermal Energy

Residual: $\quad 2.2$

Water Residuals

Recipient: 3.1

Physical and Chemical Domain

Potential Environmental Impact: A. Exploration MODERATE

B. Construction SEVERE

C. Exploitation SEVERE

D. Transformàtion SEVERE

E. Transport MINOR

F. Waste disposal/processing SEVERE

G. Termination MODERATE

H. Induced technology UNASSESSED

Example Impacts: D. Water Cooling Towers Change Local Air Movement Pattern

Data Available: 1. GRID

2. NAWDEX

3. ENDEX

4. EMDB

5. IVEP

6. AEROS

7. STORET

8. LBL-3232

9. GSA/GARRISON

10. NY/NF. . $=?$ ?

11. CDWR-143-7

12. TAMU/WHITING
13. OSU/BODVARSSON

14. NMIMT/SUMMERS

15. USGS/YOUNG

16. USGS/WILLEY

17. GEOTHERM

18. USDA/DF-73-019

19. USDA / DF -73-020

20. BLM/DF -73-005

21. ANCR - 1213

22. NOAA DRS

23. ERD $\Lambda-2533$
Models Available: 1. SUWQI

2. WQELR

3. HWQRB

4. PERCOL

5. GRAPES

6. ESPM
7. EEIED

8.- GRID

9. NBS/DF-73-012

10. USGS/HELM

11. IGS/CARTWRIGHT

12. USGS/NATHENSON

13. USGS/FOURNIER

14. USGS/TRUESDELL. 
Technology: $\quad 1.3 .2$

Geopower

Geothermal Energy

Residual: 2.2

Water Residuals

Recipient: 3.1 .1

Physical and Chemical Domain

Earth

Potential Environmental Impac's: A. Exploration MINOR

B. Construction SEVERE

C. Exploitation SEVERE

D. Transformation MINOR

E. Transport MINOR

F. Waste disposal/processing SEVERE

G. Termination MODERATE

H. Induced technology UNASSESSED

Example Impacts: E. Consumption of Resources (Steel) for Pipeline Manufacture

Data Available: 1. GRID

2. IVEP

3. GSA/GARRISON

4. TAMU/WHITING

5. OSU/BODVARSSON

6. NMIMT/SUMMERS

7. USGS/YOUNG

8. USGS/WILLEY

9. GEOTHERM

10. STORET
11. USDA / DF -73-019

12. USDA / DF -73-020

13. BLM/DF-73-005

14. ERDA-2533

Models Available: 1. WQELR

2. HWQRB

3. PERCOL

4. USGS/HELM

5. IGS/CARTWRIGHT

6. USGS/FOURNIER

7. USGS/TRUESDELL 
Technology: $\quad 1.3 .2$

Geopower

Geothermal Energy

Residual: 2.2

Water Residuals

Recipient: 3.1 .1 .1

Physical and Chemical Domain

Earth

Topography

Potential Environmental Impact: A. Exploration NONE

B. Construction NONE

C. Exploitation NONE

D. Transformation NONE

E. Transport MINOR

F. Waste disposal/processing NONE

G. Termination NONE

$\mathrm{H}$. Induced technology NONE

Example Impacts: E. Alterations to Topography due to Laying of Pipeline

Data Available:

Models Available: 
Technology: $\quad 1.3 .2$

Geopower

Geothermal Energy

Residual: $\quad 2.2$

Water Residuals

Recipient: 3.1 .1 .2

Physical and Chemical Domain

Earth

Resources

Potential Environmental Impact: A. Exploration MINOR

B. Construction MODERATE

C. Exploitation SEVERE

D. Transformation MINOR

E. Transport MINOR

F. Waste disposal/processing SEVERE

G. Termination MODERATE

H. Induced technology UNASSESSED

Example Impacts: 1. C. Withdrawal of Reservoir Water Depletes

Geothermal Resource

2. F. Reinjection of Brine May Clog Aquifer

Data Available: 1. IVEP

2. GRID

3. GEOTHERM

Models Available: 1. IGS/CARTWRIGHT

2. USGS/FOURNIER 
Technology: $\quad 1.3 .2$

Geopower

Geothermal Energy

Residual: 2.2 .

Water Residuals

Recipient: 3.1 .1 .3

Physical and Chemical Domain

Earth

Slow Processes

Potential Environmental Impact: A. Exploration MINOR

B. Construction SEVERE

C. Exploitation SEVERE

D. Transformation NONE

E. Transport NONE

F. Waste disposal/processing SEVERE

G. Termination MODERATE

H. Induced technology UNASSESSED

Example Impacts: G. Regrading on Termination may Produce Siltation and ${ }^{\circ}$ Deposition

Data Available: 1. GRID

2. IVEP

3. TAMU/WHITING

Models Available: USGS/HELM 
Technology: $\quad 1.3 .2$

Geopower

Geothermal Energy

Residual: $\quad 2.2$

Water Residuals

Recipient: 3.1 .1 .3 .1

Physical and Chemical Domain

Earth

Slow Processes

Weathe ring

Potential Environmental Impact: A. Exploration NONE

B. Construction MINOR

r. Fixplnitation MINOR.

D. Transformation NONE

E. Transport NONE

F. Waste disposal/processing MINOR

G. Termination NONE

H. Induced technology UNASSESSED

Example Impacts: A, B, C. Disposal of Wastewater Promotes Chemical Weathering

Data Available: GRID

Models Available: 
Technology: $\quad 1.3 .2$

Geopower

Geothermal Energy

Residual: 2.2

Water Residuals

Recipient: 3.1 .1 .3 .2

Physical and Chemical Domain

Earth

Slow Processes

Solution

Potential Environmental Impact: A. Exploration MINOR

B. Construction MINOR

C. Exploitation MINOR

D. Transformation NONE

E. Transport NONE

F. Waste disposal/processing MINOR

G. Termination NONE

H. Induced technology UNASSESSED

Example Impacts: A, B, C, F. Leaching of Soils by Uncontained Water

Data Available: GRID

Models Available: 
Technology: $\quad 1.3 .2$

Geopower

Geothermal Energy

Residual: $\quad 2.2$

Water Residuals

Recipient: $3.1 \cdot 1.3 .3$

Physical and Chemical Domain

Earth

Slow Processes

Sorption/Complexing

Potential Environmental Impact: A. Exploration MINOR

B. Construction MINOR

C. Exploitation SEVERE

D. Transformation NONE

E. Transport NONE

F. Waste disposal/processing. SEVERE

G. Termination NONE

$\mathrm{H}$. Induced technology NONE

Example Impacts: 1. C,F. Sorption of Trace Metals by Clay Pond Liners

2. C,F. Complexing of Methyl Mercury in Surface Waters

Data Available: GRID

Models Available: 
Technology: $\quad 1.3 .2$

Geopower

Geothermal Energy

Residual: 2.2

Water Residuals

Recipient: 3.1 .1 .3 .4

Physical and Chemical Domain

Earth

Slow Processes

Deposition

Potential Environmental Impact: A. Exploration MINOR

B. Construction SEVERE

C. Exploitation SEVERE

D. Transformation NONE

E. Transport NONE

F. Waste disposal/processing SEVERE

G. Termination MODERATE

H. Induced technology UNASSESSED

Example Impacts: 1. B, C. Siltation and Deposition of Construction Spoils

2. F. Deposition of Salts from Impounded Brine

Data Available: GRID

Models Available: 
Technology: $\quad 1.3 .2$

Geopower

Geothermal Energy

Residual: 2.2

Water Residuals

Recipient: 3.1 .1 .3 .6

Physical and Chemical Domain

Earth

Slow Processes

Subsidence

Potential Environmental Impací: A. Exploration NONE

B. Construction NONE

C. Exploitation SEVERE

D. Transformation NONE

E. Transport NONE

F. Waste disposal/processing SEVERE

G. Termination NONE

$\mathrm{H}$. Induced technology UNASSESSED

Example Impacts: 1. C. Geothermal Fluid Withdrawal may Cause Land Subsidence

2. F. Reinjection of Wastewater May Halt Subsidence

Data Available: 1. GRID

2. IVEP

3. TAMU/WHITING

Models Available: USGS/HELM 
Technology: $\quad 1.3 .2$

Geopower

Geothermal Energy

Residual: 2.2

Water Residuals

Recipient: 3.1 .1 .4

Physical and Chemical Domain.

Earth

Rapid Processes

Potential Environmental Impact: A. Exploration MINOR

B. Construction MODERATE

C. Exploitation SEVERE

D. Transformation NONE

E. Transport MINOR

F. Waste disposal/processing SEVERE

G. Termination MINOR

H. Induced technology UNASSESSED

Example Impacts: E. Pipeline Failure May Cause Erosion

Data Available: 1. GRID

2. IVEP

3. USDA / DF-073-019

4. USDA / DF -73-020

5. $\mathrm{BLM} / \mathrm{DF}-73-005$

Models Available: 
Technology: 1.3 .2

Geopower

Geothermal Energy

Residual: . 2.2

Water Residuals

Recipient: 3.1 .1 .4 .1

Physical and Chemical Domain

Earth

Rapid Processes

Flood

Potential Environmental Impact: A. Exploration NONE

B. Construction NONE

C. Exploitation MODERATE

D. Transformation NONE

E. Transport MINOR

F. Waste disposal/processing MODERATE

G. Termination NONE

H. Induced technology UNASSESSED

Example Impacts: 1. C,F. Wastewater Disposal May Kill Vegetation and Promote. Flooding

2. E. Rupture of Pipeline May Cause Local Flooding

Data Available: 1. USDA/DF-73-019

2. USDA / DF -73-020

Models Available: 
Technology:

1.3 .2

Geopower

Geothermal Energy

Residual: $\quad 2.2$

Water Residuals

Recipient: 3.1 .1 .4 .2

Physical and Chemical Domain

Earth

Rapid Processes

Erosion

Potential Environmental Impact: A. Exploration MINOR

B. Construction MODERATE

C. Exploitation MODERATE

D. Transformation NONE

E. Transport MINOR

F. Waste disposal/processing MODERATE

G. Termination MINOR

H. Induced technology UNASSESSED

Example Impacts: $\quad$ l. B, C, F. Unregulated Water Disposal May Produce Erosion

2. F. Wastewater Disposal May Kill Vegetation Resulting in Erosion

Data Available: 1. BLM/DF-73-005

Models Available: 
Technology: $\quad 1.3 .2$

Geopower

Geothermal Energy

Residual: $\quad 2.2$

Water Residuals

Recipient: 3.1.1.4.4

Physical and Chemical Domain

Earth

Rapid Processes

Landslide/Avalanche

Potential Environmental Impact: A. Exploration MINOR

B. Construction MINOR

C. Exploitation MINOR

D. Transformation NONE

E. Transport NONE

F. Waste disposal/processing MINOR

G. Termination NONE

$\mathrm{H}$. Induced technology NONE

Example Impacts: A, B, C, F. Saturation of Soil by Unregulated Water Disposal May Promote Landslide

Data Ávailable:

Models Available: 
Technology: $\quad 1.3 .2$

Geopower

Geothermal Energy

Residual: 2.2

Water Residuals

Recipient: 3.1 .1 .4 .5

Physical and Chemical Domain

Earth

Rapid Processes

Seismicity

Potential Environmental Impact: A. Exploration NONE

B. Construction NONE

C. Exploitation SEVERE

D. Transformation NONE

E. Transport NONE

F. Waste disposal/processing SEVERE

G. Termination NONE

$\mathrm{H}$. Induced technology NONE

Example Impacts: 1. C,F. Reinjection of Geothermal Fluids May Lubricate Faults

2. C. Hydraulic Splitting of Hot Dry Rock May Promote Seismicity

Data Available: 1. GRID

2. IVEP ।

Models Available: 
Technology: $\quad 1.3 .2$

Geopower

Geothermal Energy

Residual: 2.2

Water Residuals

Recipient: 3.1 .1 .5

Physical and Chemical Domain

Earth

Unique Features

Potential Environmental Impact: A. Exploration MINOR

B. Construction MINOR

C. Exploitation SEVERE

D. Transformation NONE

E. Transport NONE

F. Waste disposal/processing SEVERE

G. Termination NONE

H. Induced technology UNASSESSED

Example Impacts: C. Geothermal Field,as a Unique Feature, is Depletable by Water Extraction

Data Available: 1: GRID

2. IVEP

3. STORET

4. GSA/GARRISON

5. OSU/BODVARSSON

6. GEOTHERM

7. ERDA -2533

Models Available: 1. WQELR

2. HWQRB

3. PERCOL

4. USGS/TRUESDELL 
Technology: $\quad 1.3 .2$

Geopower

Geothermal Energy

Residual: 2.2

Water Residuals

Recipient: 3.1 .1 .5 .1

Physical and Chemical Domain

Earth

Unique Features

Aquifer Recharge

Potential Environmental Impact: A. Exploration MINOR

B. Construction MINOR

C. Exploitation SEVERE

D. Transformation . NONE

E. Transport NONE

F. Waste disposal/processing SEVERE

G. Termination NONE

H. Induced technology UNASSESSED

Example Impacts: 1: B, F. Geothermal Fluids May Contaminate Adjacent Aquifers

2. F. Geothermal Fluid May be Reinjected for Aquifer Recharge

Data Available: 1. GRID

2. IVEP

3. STORET

4. OSU/BODVARSSON

5. ERDA-2533

Models Available: 1. WQELR

2. HWQRB

3. PERCOL 
Technology: 1.3 .2

Geopower

Geothermal Energy

Residual: 2.2

Water Residuals

Recipient: 3.1 .2

Physical and Chemical Domain

Water

Potential Environmental Impact: A. Exploration MODERATE

B. Construction SEVERE

C. Exploitation SEVERE

D. Transformation MINOR

E. Transport NONE

F. Waste disposal/processing SEVERE

G. Termination MODERATE

H. Induced technology UNASSESSED

Example Impacts: 1. A, B, G. Contamination of Surface or Ground Water by Drilling Mud Drainings

2. C. Contamination of Surface or Ground Water by Leakage from Geothermal Fluid Circuit

Data Available: 1. GRID

2. NAWDEX

3. ENDEX

4. EMDB

5. IVEP

6. STORET

7. LBL- 3232

8. NY/DEC-70

9. NMIMT/SUMMERS
10. CDWR $-143-7$

11. USGS/YYOUNG

12. USGS/WILLEY

13. GEOTHERM

14. ANCR-1213

15. NOAA/DRS

Models Available: 1. SUWQI

2. WQELR

3. HWQRB

4. PERCOL

5. GRAPES

6. ESPM

7. EEIED

8. NBS-DF-73-012

9. USGS/NATHENSON 
Technology:

1.3 .2

Geopower

Geothermal Energy

Residual: $\quad 2.2$

Water residuals

Recipient: $\quad 3.1 .3$

Physical and chemical domain

Air

Potential Environmental Impact: A. Exploration MINOR

B. Construction MINOR

C. Exploitation SEVERE

D. Transformation SEVERE

E. Transport NONE

F. Waste disposal/processing. SEVERE

G. Termination MINOR

H. Induced technology UNASSESSED

Example Impacts: 1. D. Addition of Water Vapor to Air from Cooling Tower

2. D. Addition of Hydrogen Sulfide to Air from Cooling Tower

Data Available: 1. GRID

2. AEROS

3. $E M D B$

4. IVEP

5. LBL - 3232

6. GEOTHERM

Models Available: 
Technology: $\quad 1.3 .2$

Geopower

Geothermal Energy

Residual: $\quad 2.2$

Water residuals.

Recipient: $\quad 3.2$

Biological domain

Potential Environmental Impact: A. Exploration MODERATE

B. Construction SEVERE

C. Exploitation SEVERE

D. Transformation MODERATE

E. Transport MODERATE

F.. Waste disposal/processing SEVERE

G. Termination SEVERE

H. Induced technology UNASSESSED

Example Impacts: 1. E. Pipeline Construction Destroys Vegetation

2. A, B, C. Preemption of Surface Water Resources Deprives Animals

Data Available: 1. GRID

2. NAWDEX

3. ENDEX

4. IVEP

5. STORET

6. UM/SMITH

7. IU/ZEIKUS

8. ERDA -2892

Models Available: 
Technology: 1.3 .2

Geopower

Geothermal Energy

Residual: $\quad 2.2$

Water residuals

Recipient: 3.2 .1

Biological domain

Animals

Potential Environmental Impact: A. Exploration MODERATE

B. Construction SEVERE

C. Exploitation SEVERE

D. Transformation MODERATE

E. Transport MINOR

F. Waste disposal/processing SEVERE

G. Termination SEVERE

H. Induced technology UNASSESSED

Example Impacts: 1. A, B, C, D, F. Contamination of Drinking Water by Toxic Effluents

2. A, B, C. Fish Kills from Water Siltation

Data Available: 1. GRID

2. NAWDEX

3. ENDEX

4. IVEP

5. STORET

6. UM/SMITH

Models Available: 
Technology: $\quad 1.3 .2$.

Geopower

Geothermal Energy

Residual: $\quad 2.2$

Water residuals

Recipient: $\quad 3.2 .2$

Biological domain

Plants

Potential Environmental Impact: A. Exploration MODERATE

B. Construction SEVERE

C. Exploitation SEVERE

D. Transformation MODERATE

E. Transport MODERATE

F. Waste disposal/processing SEVERE

G. Termination SEVERE

$\mathrm{H}$. Induced technology UNASSESSED

Example Impacts: 1. A, C, F. Killing of Vegetation by Salts in Brine

2. A, C, F. Killing of Vegetation by Thermal Releases from Geothermal Fluids

Data Available: $\quad$ 1. GRID

2. NAWDEX

3. ENDEX

4. IVEP

5. STORET

6. IU / ZEIKUS

7. ERDA-2892

Models Available: 
Technology: $\quad 1.3 .2$

Geopower

Geothermal Energy

Residual: $\quad 2.2$

Water residuals

Recipient: $\quad 3.3$

Social-Cultural Domain

Potential Environmental Impac's: A. Exploration MODERATE

B. Construction SEVERE

C. Exploitation SEVERE

D. Transformation MODERATE

E. Transport MODERATE

F. Waste disposal/processing SEVERE

G. Termination SEVERE

H. Induced technology UNASSESSED

Example Impacts: 1. E. Preemption of Land Use for Construction of Pipelines

Data Available: 1. GRID

2. ENDEX

3. IVEP

Models Available:

.7 
Technology: $\quad 1.3 .2$

Geopower

Geothermal Energy

Residual: $\quad 2.2$

Water residuals

Recipient: 3.3 .1

Social-Cultural domain

Land use

Potential Environmental Impac's: A. Exploration MINOR

B. Construction SEVERE

C. Exploitation SEVERE

D. Transformation SEVERE

E. Transport MODERATE

F. Waste disposal/processing SEVERE

G. Termination SEVERE

$H$. Induced technology UNASSESSED

Example Impacts: 1. A, B, C, D, F. Preemption of Prior Use by Construction of Cooling Ponds :

2. G. Lowering of Irrigation Wells by Withdrawal from Aquifer

Data Available: $\quad$ 1. GRID

2. ENDEX

3. IVEP

Models Available: 
Technology: 1.3 .2

Geopower

Geothermal Energy

Residual: $\quad 2.2$

Water residuals

Recipient: 3.3 .2

Social-Cultural domain

Recreation

Potential Environmental Impací: A. Exploration MINOR

B. Construction SEVERE

C. Exploitation SEVERE

D. Transformation NONE

E. Transport MINOR

F. Waste disposal/processing SEVERE

G. Termination SEVERE

H. Induced technology UNASSESSED

Example Impacts: 1. A, B, C, F. Killing of Game Fish by Thermal Releases from Geothermal Fluids

2. A, C, F. Impairment of Recreational Boating by Releases of

Data Available: 1. GRID Brine

2. ENDEX

3. IVEP

Models Available: 
Technology: 1.3 .2 Geopower

Geothermal Energy

Residual: $\quad 2.2$

Water residuals

Recipient: $\quad 3.3 .3$

Social-Cultural domain

Aesthetics

Potential Environmental Impact: A. Exploration MINOR

B. Construction SEVERE

C. Exploitation SEVERE

D. Transformation MINOR

E. Transport MINOR

F. Waste disposal/processing SEVERE

G. Termination SEVERE

H. Induced technology UNASSESSED

Example Impacts: 1. A, B, C, F. Impairment of Landscape by Dead Vegetation Resulting from Brine Damage

2. A, B, C, F. Loss of Aesthetic Enjoyment of Mineral Springs

Data Available: 1. GRID

2. ENDEX

Models Available: 
Technology: 1.3 .2

Geopower

Geothermal Energy

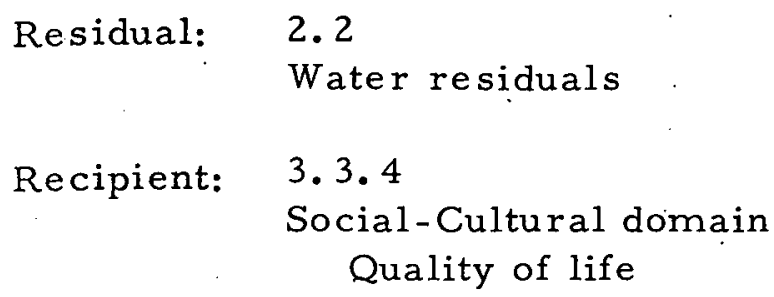

Residual: 2.2

Water residuals

Recipient: 3.3 .4

Social-Cultural domain

Quality of life

Potential Environmental Impact: A. Exploration MODERATE

B. Construction SEVERE

C. Exploitation SEVERE

D. Transformation MODERATE

E. Transport MINOR

F. Waste disposal/processing SEVERE

G. Termination MODERATE

!?

$H$. Induced technology UNASSESSED

Example Impacts: 1. A, B, C, D, E. Safety Hazard of High-Pressure Steam

$\begin{array}{ll}\text { Data Available: } & \text { 1. GRID } \\ & \text { 2. ENDEX } \\ & \text { 3. IVEP }\end{array}$

Models Available: 
Technology: $\quad 1.3 .2$

Geopower

Geothermal Energy

Residual: $\quad 2.2$

Water residuals

Recipient: $\quad 3.3 .4 .1$

Social-Cultural domain

Quality of life

Health and Safety

Potential Environmental Impact: A. Exploration MODERATE

B. Construction SEVERE

C. Exploitation SEVERE

D. Transformation NODERATE

F. Transport MINOR

F. Waste disposal/processing SEVERE

G. Termination NONE

$\mathrm{H}$. Induced technology UNASSESSED

Example Impacts:1. A, B, C, D, E. Safety Hazard Due to Corrosivity of Hot Brine

2. F. Health Hazard Due to Trace Metals in Waste Water

Data Available: 1. GRID

2. ENDEX

3. IVEP

Models Available: 
Technology: $\quad 1.3 .2$

Geopower

Geothermal Energy

Residual: $\quad 2.2$

Water residuals

Recipient: 3.3 .4 .2

Social-Cultural domain

Quality of life

Life style

Potential Environmental Impact: A. Exploration NONE

B. Construction NONE

C. Exploitation MODERATE

D. Transformation NONE

E. Transport NONE

F. Waste disposal/processing MODERATE

G. Termination MODERATE

$\mathrm{H}$. Induced technology UNASSESSED

Example Impacts: 1. C,F. Loss of Game or Commercial Fish May Affect Local Indian Populations

Data Available:

Models Available: 
Technology: 1.3 .2

Geopower

Geothermal Energy

Residual: 2.2

Water residuals

Recipient: $\quad 3.3 .5$

Social-Cultural domain

Legal aspects

Potential Environmental Impact: A. Exploration MINOR

B. Construction SEVERE

C. Exploitation MODERATE

D. Transformation NONE

E. Transport MODERATE

F. Waste disposal/processing SEVERE

G. Termination NONE

H. Induced technology UNASSESSED

Example Impacts: 1. B,E. Licenses \& Permits to Discharge Geothermal Fluids

2. E. Pipeline Rights of Way

Data Available: $\quad$ 1. GRID

2. IVEP

Models Available: 
Technology: $\quad 1.3 .2$

Geopower

Geothermal Energy

Residual: $\quad 2.2$

Water residuals

Recipient: $\quad 3.4$

Economic domain

Potential Environmental Impact: A. Exploration .MINOR

B. Construction SEVERE

C. Exploitation SEVERE

D. Transformation NONE

E. Transport MINOR

F. Waste disposal/processing SEVERE

G. Termination NONE

H. Induced technology UNASSESSED

Example Impacts: 1. F. Recoverable Brine Salts May be Economic Asset

Data Available: 1. GRID

2. IVEP

Models Available:

B -65 
Technology: $\quad 1.3 .2$

Geopower

Geothermal Energy

Residual: $\quad 2.2$

Water residuals

Recipient: $\quad 3.4 .1$

Economic domain

Demographic factors

Potential Environmental Impact: A. Exploration MINOR

B. Construction SEVERE

C. Exploitation SEVERE

D. Transformation NONE

E. Transport NONE

F. Waste disposal/processing SEVERE

G. Termination NONE

H. Induced technology UNASSESSED

Example Impacts: 1. A, B, C, F. Cleanup Costs to Municipal Water Supply

2. B, C,E. Additional Work Force Required for Pipeline Construction Crews

Data Available:

GRID

Models Available: 
Technology: $\quad 1.3 .2$

Geopower

Geothermal Energy

Residual: 2.2

Water Residuals

Recipient: 3.4 .2

Economic Domain

Infrastructure Domain

Potential Envixonmental Impact: A. Exploration MINOR

B. Construction SEVERE

C. Exploitation SEVERE

D. Transformation NONE

E. Transport MINOR

F. Waste disposal/processing SEVERE

G. Termination NONE

H. Induced technology UNASSESSED

Example Impacts: 1. A, B, C, F. Added Burden on Local Waste Disposal and Sewage Systems.

2. E. Pipeline Effects on Local Road/Railroad Systems

Data Available: 1. GRID

2. IVEP

Models Available: 
4 
Technology: $\quad 1.3 .2$

Geopower

Geothermal Energy

Residual: 2.3

Atmospheric Residuals

Recipient: 3.1

Physical and Chemical Domain

Potential Environmental Impac's: A. Exploration MINOR

B. Construction MODERATE

C. Exploitation SEVERE

D. Transformation MODERATE

E. Transport MINOR

F. Waste disposal/processing SEVERE

G. Termination MINOR

H. Induced technology. UNASSESSED

Example Impacts: 1. A, C, D, F. Some Residuals Gases - Hydrogen Sulfide, Ammonia, Carbon Dioxide, Methane, Mercury Vapor - May be Released to Atmosphere

Data Available: 1. GRID

2. ENDEX

3. IVEP

4. AEROS

5. GEOTHERM

6. STORET

7. APSFBA

8. TRRP

9. $\mathrm{COM} / \mathrm{DF}-73-009$

10. LBL-3232

11. ERDA-2877

Models Available: 1. ADPIC

2. ARAC

3. EEIED

4. LIRAQ 1

5. SMICK

6. MESODIFF

7. BESOM

8. GRAPES

9. ESPM

10. LIRAQ 2 
Technology: $\quad 1.3 .2$

Geopower

Geothermal Energy

Residual: $\quad 2.3$

Atmospheric residuals

Recipient: 3.1.1

Physical and chemical domain

Earth

Potential Environmental Impact: A. Exploration NONE

B. Construction NONE

C. Exploitation MINOR

D. Transformation NONE

E. Transport NONE

F. Waste disposal/processing MODERATE

G. Termination NONE

H. Induced technology UNASSESSED

Example Impacts: 1. C, F. Fabrication of air pollution control equipment consumes steel, chemical resources

Data Available:

GRID

Models Available: 1. ADPIC

2. ARAC

3. MESODIFF

4. $\operatorname{LIRAQ} 1$

5. SMICK

6. LIRAQ 2 
Technology: $\quad 1.3 .2$

Geopower

Geothermal Energy

Residual: 2.3

Atmospheric residuals

Recipient: $\quad 3.1 .1 .2$

Physical and chemical domain

Earth

Resources

Potential Environmental Impact: A. Exploration NONE

B. Construction NONE

C. Exploitation NONE

D. Transformation NONE

E. Transport NONE

F. Waste disposal/processing MODERATE

G. Termination NONE

$\mathrm{H}$. Induced technology UNASSESSED

Example Impacts: 1. F. Control of noxious gases from condensate water consumes chemicals

2. F. Soil may be contaminated by airborne salts from brine ponds

Data Available:

Models Available: 
Technology: $\quad 1.3 .2$

Geopower

Geothermal Energy

Residual: $\quad 2.3$

A tmospheric residuals

Recipient: 3.1.1.3

Physical and chemical domain

Earth

Slow processes

Potential Environmental Impact: A. Exploration NONE

B. Construction. NONE

C. Exploitation MINOR

D. Transformation NONE

E. Transport NONE

F. Waste disposal/processing MINOR

G. Termination NONE

H. Induced teshnolngy UNASSESSED

Example Impacts: 1. C,F. Corrosive residuals such as sulfuric acid may be scrubbed from atmosphere and contribute to erosion

Data Available:

Models Available: 
Technology: 1.3 .2

Geopower

Geothermal Energy

Residual: $\quad 2.3$

A tmospheric residuals

Recipient: 3.1.1.3.1

Physical and chemical domain

Earth

Slow processes

Weathering

Potential Environmental Impaci: A. Exploration NONE

B. Construction NONE

C. Exploitation NONE

D. Transformation NONE

E. Transport NONE

F. Waste disposal/proceșsing MINOR

G. Termination NONE

$\mathrm{H}$. Induced technology NONE

Example Impacts: 1. C. Rainfall acidified by hydrogen sulfide oxidation products may promote chemical weathering.

Data Available:

Models Available:

$\mathrm{B}-73$ 
Technology: $\quad 1.3 .2$

Geopower

Geothermal Energy

Residual: 2.3

Atmospheric residuals

Recipient: 3.1 .1 .3 .2

Physical and chemical domain

Earth

Slow processes

Solution

Potential Environmental Impact: A. Exploration

NONE

B. Construction

NONE

C. Exploitation

MINOR

D. Transformation

NONE

E. Transport

NONE

F. Waste disposal/processing

MINOR

G. Termination

NONE

H. Induced technology

NONE

Example Impacts: 1. C. Rainfall acidified by hydrogen sulfide oxidation products may promote leaching of solubles from soil.

Data Available:

Models Available: 
Technology: $\quad 1.3 .2$

Geopower

Geothermal Energy

Residual: 2.3

Atmospheric residuals

Recipient: 3.1 .1 .3 .3

Physical and chemical domain

Earth

$$
\begin{aligned}
& \text { Slow processes } \\
& \text { Sorption/Complexing }
\end{aligned}
$$

Potential Environmental Impact: A. Exploration

NONE

B. Construction

NONE

C. Exploitation

MINOR

D. Transformation

NONE

E. Transport

NONE

F. Waste disposal/processing

MINOR

G. Termination

NONE

$\mathrm{H}$. Induced technology

UNASSESSED

Example Impacts: 1. C,F. Soluble gases (Ammonia, Hydrogen Sulfide) in cooling tower drift are redeposited and sorbed by soils.

Data Available:

Models Available: 
Technology: $\quad 1.3 .2$

Geopower

Geothermal Energy

Residual: 2.3

Atmospheric residuals

Recipient: 3.1 .2

Physical and chemical domain

Water

Potential Environmental Impact: A. Exploration

MINOR

B. Construction

MINOR

C. Exploitation

MODERATE

D. Transformation

MODERATE

E. Transport

NONE

F. Waste disposal/processing

MODERATE

G. Termination

NONE

$\mathrm{H}$. Induced technology

NONE

Example Impacts: 1. A, B, C, F. Scrubbing of airborne emissions into surface and ground waters

2. A, B, C, F. Fallout of particulates into surface waters

Data Availablè: 1. GRID

2. STORET

3. LBL-3232

Models Available: SMICK 
Technology: 1.3 .2

Geopower

Geothermal Energy

Residual: 2.3

Atmospheric residuals

Recipient: $\quad 3.1 .3$

Physical and chemical domain

Air

Potential Environmental Impact: A. Exploration

MINOR

B. Construction

MODERA TE

C. Exploitation

SE VERE

D. Transformation

MODERA TE

E. Transport

MINOR

F. Waste disposal/processing

SE VERE

G. Termination

MINOR

H. Induced technology

UNASSESSED

Example Impacts: 1. A, C, D, F. Degradation of air quality from geothermal gases.

2. A, B, C, E, G. Emissions from vehicles.

Data Available: 1. GRID

2. AEROS

3. ENDEX

4. IVEP

5. GEOTHERM

6. APSFBA

7. TRRP

8. COM/DF - $73-009$

9. LBL-3232

10. ERDA - 287.7

Models Available: 1. BESOM

2. GRAPES

3. ESPM

4. EEIED 
Technology: $\quad 1.3 .2$

Geopower

Geothermal Energy

Residual: $\quad 2.3$

Atmospheric residuals

Recipient: $\quad 3.2$

Biological domain

Potential Environmental Impact: A. Exploration

MINOR

B. Construction

SE VERE

C. Exploitation

SEVERE

D. Transformation

MODERATE

E. Transport

MODERATE

F. Waste disposal/processing

SEVERE

G. Termination

MODERATE

H. Induced technology

UNASSESSED

Example Impacts: 1. F. Mercury and selenium volatilized and damage aquatic food chain.

Data Available: 1. GRID

2. ENDEX

3. IVEP

4. UM/SMITH

Models Available: 
Technology: 1.3 .2

Geopower

Geothermal Energy

Residual: 2.3

A tmospheric residuals

Recipient: $\quad 3.2 .1$

Biological domain

Animals

Potential Environmental Impact: A. Exploration

MINOR

B. Construction

SEVERE

C. Exploitation

SEVERE

D. Transformation

MINOR

E. Transport

MINOR

F. Waste disposal/processing

MODERATE.

G. Termination

MODERATE

H. Induced technology

UNASSESSED

Example Impacts: 1. A, B, C, F. Noxious fumes from geothermal source harm or displace wildlife.

Data Available: 1. GRID

2. ENDEX

3. IVEP

4. UM/SMITH

Models Available: 
Technology: $\quad 1.3 .2$

Geopower

Geothermal Energy

Residual: $\quad 2.3$

Atmospheric residuals

Recipient: $\quad 3.2 .2$

Biological domain

Plants

Potential Environmental Impact: A. Exploration

MINOR

B. Construction

SE VERE

C. Exploitation

SEVERE

D. Transformation

MODERATE

E. Transport

MODERATE

F. Waste disposal/processing

SEVERE

G. Termination

MODERATE

H. Induced technology

UNASSESSED

Example Impacts: 1. A, B, C, F. Noxious fumes from geothermal source damage vegetation.

2. B, G. Dust from grading damages vegetation.

Data Available: 1. GRID

2. ENDEX

3. IVEP

Models Available: 
Technology: $\quad 1.3 .2$

Geopower

Geothermal Energy

Residual: 2.3

Atmospheric residuals

Recipient: $\quad 3.3$

Social-Cultural domain

Potential Environmental Impac'c:
A. Exploration
B. Construction
C. Exploitation
D. Transformation
E. Transport
F. Waste disposal/processing
G. Termination
H. Induced technology

MINOR

SEVERE

SE VERE

SE VERE

MODERATE

SEVERE

MINOR

UNASSESSED

Example Impacts: 1. C, D, F. Air emissions such as hydrogen sulfide and ammonia . may preempt land use.

Data Available:

1. GRID

2. ENDEX

3. IVEP

4. AEROS

Models Available: 1. UH/INDUSTRY

2. EEPM 
Technology: $\quad 1.3 .2$.

Geopower

Geothermal Energy

Residual: 2.3

A tmospheric residuals

Recipient: 3.3 .1

Social-Cultural domain

Land use.

Potential Environmental Impact: A. Exploration

MINOR

B. Construction

MINOR

C. Exploitation

SEVERE

D. Transformation

SE VERE

E. Transport

MINOR

F. Waste disposal/processing

MODERATE

G. Termination

MINOR

H. Induced technology

UNASSESSED

Example Impacts: 1. A, C, D, F. Noxious fumes limit land use for grazing, agriculture, recreation.

Data Available: 1. GRID

2. ENDEX

3. IVEP

Models Available: 
Technology: $\quad 1.3 .2$

Geopower

Geothermal Energy

Residual: 2.3

Atmospheric residuals

Recipient: $\quad 3.3 .2$

Social-Cultural domain

Recreation

Potential Environmental Impac's: A. Exploration

MINOR

B. Construction

MINOR

C. Exploitation

MODERA TE

D. Transformation

MODERATE

E. Transport

MINOR

F. Waste disposal/processing

MODERATE

G. Termination

MINOR

$\mathrm{H}$. Induced technology

UNASSESSED

Example Impacts: 1. A, C, D, F. Noxious fumes from geothermal source affect recreation.

2. E, G. Emissions from vehicles affect recreation.

Data Available: 1. GRID

2. ENDEX

3. IVEP

Models Available: 
Technology: $\quad 1.3 .2$

Geopower

Geothermal Energy

Residual: $\quad 2.3$

Atmospheric residuals

Recipient: $\quad 3.3 .3$

Social-Cultural domain

Aesthetics

Potential Environmental Impact: A. Exploration

MINOR

B. Construction

MODERATE

C. Exploitation

SE VERE

D. Transformation

SE VERE

E. Transport

MODERATE

F. Waste disposal/processing

SEVERE

G. Termination

MINOR

H. Induced technology

UNASSESSED

Example Impacts: 1. A, C, D, F. Noxious fumes from geothermal source impair enjoyment of wild areas

2. B, G. Dust from grading impairs enjoyment of outdoor prospects.

Data Available: 1. GRID

2. ENDEX

3. AEROS

4. IVEP

Models Available: 
Technology: $\quad 1.3 .2$

Geopower

Geothermal Energy

Residual: $\quad 2.3$

Atmospheric residuals

Recipient: $\quad 3.3 .4$

Social-Cultural domain

Quality of life

Potential Environmental Impaci: A. Exploration

MINOR

B. Construction

MINOR

C. Exploitation

SE VERE

D. Transformation

SE VERE

E. Transport

MODERATE

F. Waste disposal/processing

SEVERE

G. Termination

MINOR

$\mathrm{H}$. Induced technology

UNASSESSED

Example Impacts: 1. C, D. Inherent hazard of. working with gases under pressure.

Data Available: 1. GRID

2. ENDEX

3. AEROS

4. IVEP

Models Available: 
Technology: $\quad 1.3 .2$

Geopower

Geothermal Energy

Residual: $\quad 2.3$

Atmospheric residuals

Recipient: $\quad 3.3 .4 .1$

Social-Cultural domain

Quality of life

Health and safety

Potential Environmental Impact: A. Exploration

MINOR

B. Construction

MINOR

C. Exploitation

SEVERE

D. Transformation

SEVERE

E. Transport

MODERATE

F. Waste disposal/processing

SEVERE

G. Termination

MINOR

H. Induced technology

UNASSESSED

Example Impacts: 1. A, C, D, F. Noxious fumes from geothermal source may be toxic.

Data Available: 1. GRID

2. ENDEX

3. IVEP

4. AEROS

Models Available: 
Technology: $\quad 1.3 .2$

Geopower

Geothermal Energy

Residual: 2.3

Atmospheric residuals

Recipient: 3.3 .4 .2

Social-Cultural domain

Quality of life

Life style

Potential Environmental Impací: A. Exploration

MINOR

B. Construction

MINOR

C. Exploitation

MINOR

D. Transformation

MINOR

E. Transport

MINOR

F. Waste disposal/processing

MINOR

G. Termination

MINOR

H. Induced technology

UNASSESSED

Example Impacts: 1. A, B, C, D, F. Effects on livestock and game may have adverse impacts on local Indian communities.

Data Available: 1. GRID.

2. ENDEX

Models Available: 
Technology: $\quad 1.3 .2$

Geopower

Geothermal Energy

Residual: $\quad 2.3$

Atmospheric residuals

Recipient: $\quad 3.3 .5$

Social-Cultural domain

Legal aspects

Potential Environmental Impací: A. Exploration

NONE

B. Construction

SE VERE

C. Exploitation

SE VERE

D. Transformation

SE VERE

E. Transport

NONE

F. Waste disposal/processing

SE VERE

G. Termination

NONE

H. Induced technology

UNASSESSED

Example Impacts: 1. B, C, D, F. Controlling agencies' air pollution requirements must be met.

Data Available: 1. GRID

2. AEROS

3. IVEP

Models Available: 1. UH/INDUSTRY

2. EEPM 
Technology: $\quad 1.3 .2$

Geopower

Geothermal Energy

Residual: 2.3

Atmospheric residuals

Recipient: $\quad 3.4$

Economic domain

Potential Environmental Impact: A. Exploration

NONE

B. Construction

C. Exploitation

D. Transformation

E. Transport

F. Waste disposal/processing

G. Termination

H. Induced technology
MINOR

MODERATE

MODERATE

MINOR

MODERATE

NONE

NONE

Example Impacts: 1. B, C, D, F. Air pollution controls require specially skilled workers.

Data Available: 1. GRID

2. IVEP

Models Available: 
Technology: $\quad 1.3 .2$

Geopower

Geothermal Energy

Residual: $\quad 2.3$

Atmospheric residuals

Recipient: 3.4 .1

Economic domain

Demographic factors

Potential Environmental Impacî: A. Exploration

NONE

B. Construction

MINOR

C. Exploitation

MODERATE

D. Transformation

MODERATE

E. Transport

MINOR

F. Waste disposal/processing

MODERA TE

G. Termination

NONE

$\mathrm{H}$. Induced technology

NONE

Example Impacts: 1. B, C, D, F. Air pollution controls will create employment opportunities.

Data Available: 1. GRID

2. IVEP

Models Available: 

Technology: 1.3 .2

Geopower

Geothermal Energy

Residual: 2.4

Biological residuals

Recipient: 3.1

Physical and chemical domain

Potential Environmental Impact: A. Exploration

MINOR

B. Construction

SE VERE

C. Exploitation

SEVERE

D. Transformation

NONE

E. Transport

MODERATE

F. Waste disposal/processing

MODERATE

G. Termination

SE VERE

H. Induced technology

UNASSESSED

Example Impacts:

Data Available: 1. NAWDEX

2. AEROS

3. STORET.

4. PARAMETRIX

Models Available: 
Technology: $\quad 1.3 .2$

Geopower

Geothermal Energy

Residual: $\quad 2.4$

' Biological residuals

Recipient: $\quad 3.1 .1$

Physical and chemical domain

Earth

Potential Environmental Impact: A. Exploration

MINOR

B. Construction

SEVERE

C. Exploitation

SE VERE

D. Transformation

NONE

E. Transport

MODERATE

F. Waste disposal/processing

MODERATE

G. Termination

SE VERE

H. Induced technology

UNASSESSED

Example Impacts: 1. E. Pipeline construction in woodland increases marginal browsers, promoting hunting.

Data Available:

Models Available: 
Technology: $\quad 1.3 .2$

Geopower

Geothermal Energy

Residual: $\quad 2.4$

Biological residuals

Recipient: 3.1 .1 .4

Physical and chemical domain

Earth

Rapid processes

Potential Environmental Impac'c: A. Exploration

MINOR

B. Construction

SEVERE

C. Exploitation

SEVERE

D. Transformation

NONE

E. Transport

MODERATE

F. Waste disposal/processing

MODERATE

G. Termination

SE.VERE

H. Induced technology

UNASSESSED

Example Impacts: 1. G. Cleared areas subject to erosion prior to reestablishment of vegetation.

Data Available:

Models Available: 
Technology: $\quad 1.3 .2$

Geopower

Geothermal Energy

Residual: $\quad 2.4$

Biological residuals

Recipient: 3.1.1.4.1

Physical and chemical domain

Earth

$$
\begin{gathered}
\text { Rapid processes } \\
\text { Flood }
\end{gathered}
$$

Potential Environmental Impact: A. Exploration

MINOR

B. Construction

SEVERE

C. Exploitation

MINOR

D. Transformation

NONE

E. Transport

MINOR

F. Waste disposal/processing

MODERATE

G. Termination

MINOR

H. Induced technology

NONE

Example Impacts: 1. A, B, C, E, F. Removal of plant cover may increase runoff rate, result in flooding.

2. G. Restoration of plant cover lessens danger of flooding.

Data Available:

Models Available: 
Technology: $\quad 1.3 .2$

Geopower

Geothermal Energy

Residual: $\quad 2.4$

B iological residuals

Recipient: 3.1 .1 .4 .2

Physical and chemical domain

Earth

Rapid processes

Erosion

Potential Environmental Impact: A. Exploration

MINOR

B. Construction

SE VERE

C. Exploitation

SE VERE

D. Transformation

NONE

E. Transport

MODERATE

F. Waste disposal/processing

MODERATE

G. Termination

SEVERE

H. Induced technology

UNASSESSED

Example Impacts: 1. A, B, C, E, F. Removal of plant cover may increase runoff rate, result in erosion

Data Available:

Models Available:

B -95 
Technology: $\quad 1.3 .2$

Geopower

Geothermal Energy

Residual: $\quad 2.4$

Biological residuals

Recipient: 3.1.1.4.4

Physical and chemical domain

Earth

$$
\begin{aligned}
& \text { Rapid processes } \\
& \text { Landslide/Avalanche }
\end{aligned}
$$

Potential Environmental Impact: A. Exploration

B. Construction

MINOR

C. Exploitation

MODERATE

D. Transformation

MODERATE

E. Transport

NONE

F. Waste disposal/processing

MINOR

G. Termination

MINOR

H. Induced technology

UNASSESSED

Example Impacts: 1. A, B, C, E, F. Removal of plant cover destabilizes slopes.

2. G. Restoration of plant cover stabilizes slopes.

Data Available:

Models Available: 
Technology: $\quad 1.3 .2$

Geopower

Geothermal Energy

Residual: 2.4

Biological residuals

Recipient: 3.1 .2

Physical and chemical domain

Water

Potential Environmental Impact: A. Exploration NONE

B. Construction NONE

C. Exploitation MINOR

D. Transformation NONE

E. Transport NONE

F. Waste disposal/processing MINOR

G. Termination NONE

H. Induced technology NONE

Example Impacts: 1. C, F. Aquatic plant growth stimulated or impaired by the rmal emissions.

Data Available: 1. NAWDEX

2. STORET

3. PARAMETRIX

Models Available: 
Technology: $\quad 1.3 .2$

Geopower

Geothermal Energy.

Residual: $\quad 2.4$

Biological residuals

Recipient: 3.2

Biological domain

Potential Environmental Impact: A. Exploration

MODERATE

B. Construction

SE VERE

C. Exploitation

SEVERE

D. Transformation

MODERA TE

E. Transport

MODERATE

F. Waste disposal/processing

MINOR

G. Termination

MODERATE

H. Induced technology

UNASSESSED

Example Impacts: 1. A, B, C, D, E, F. Loss of vulnerable species shifts ecological balance.

Data Available: 1. NAWDEX

2. ENDEX

3. PARAMETRIX

4. VTN

Models Available: 1. ORRMIS

2. CSU/HUNT

3. ESIS 
Technology: $\quad 1.3 .2$

Geopower

Geothermal Energy

Residual: $\quad 2.4$

Biological residuals

Recipient: $\quad 3.2 .1$

Biological domain

Animals

Potential Environmental Impact: A. Exploration

MODERATE

B. Construction

SEVERE

C. Exploitation

SEVERE

D. Transformation

MODERATE

E. Transport

MINOR

F. Waste disposal/processing

MINOR

G. Termination

MODERA TE

$\mathrm{H}$. Induced technology

UNASSESSED

Example Impacts: 1. A, B, C, D, E, F. Ecological balance perturbed by displacement of more mobile species.

2. G. Ecological balance slowly restored.

Data Available: $\quad$ 1. NAWDEX

2. ENDEX

3. PARAMETRIX

4. VTN

Models Available: 1. ORRMIS

2. CSU/HUNT

3. ESIS 
Technology: 1.3 .2

Geopower

Geothermal Energy

Residual: 2.4

Biological residuals

Recipient: 3.2 .2

Biological domain

Plants

Potential Environmental Impact: A. Exploration

MINOR

B. Construction

MODERA TE

C. Exploitation

MODERATE

D. Transformation

MODERATE

E. Transport

MODERATE

F. Waste disposal/processing

MINOR

G. Termination

MODERATE

H. Induced technology

UNASSESSED

Example Impacts: 1. A, B, C, D, E, F. Ecological balance perturbed by loss of. fragile species.

2. G. Ecological balance slowly restored.

Data Available: 1. NAWDEX

2. ENDEX

3. PARAMETRIX

Models Available: 1. ORRMIS

2. CSU/HUNT 
Technology: $\quad 1.3 .2$

Geopower

Geothermal Energy

Residual: $\quad 2.4$

Biological residuals

Recipient: 3.3

Social-Cultural domain

Potential Environmental Impact: A. Exploration

MINOR

B. Construction

SEVERE

C. Exploitation

SEVERE

D. Transformation

MODERA TE

E. Transport

MODERATE

F. Waste disposal/processing

SE VERE

G. Termination

MODERATE

$\mathrm{H}$. Induced technology

UNASSESSED

Example Impacts: 1. A, B, C, D, E, F, G. Shutdowns may cause malodorous fish kills.

Data Available:

Models Available: 
Technology: 1.3 .2

Geopower

Geothermal Energy

Residual: $\quad 2.4$

Biological residuals

Recipient: 3.3 .1

Social-Cultural domain

Land use

Potential Environmental Impact: A. Exploration

MINOR

B. Construction

MODERA TE

C. Exploitation

MODERATE

D. Transformation

MINOR

E. Transport

MODERA TE

F. Waste disposal/processing

MODERA TE

G. Termination

MODERATE

H. Induced technology

UNASSESSED

Example Impacts: 1. A, B, C, D, E, F. Loss of vegetation affects ranching, agriculture, forestry land use.

2. D, F. Waste heat may be used to stimulate crop production and promote agricultural land use.

Data Available:

Models Available: 
Technology: $\quad 1.3 .2$

Geopower

Geothermal Energy

Residual: 2.4

Biological residuals

Recipient: 3.3 .2

Social-Cultural domain

Recreation

Potential Environmental Impact: A. Exploration

MINOR

B. Construction

MODERA TE

C. Exploitation

SEVERE

D. Transformation

MINOR

E. Transport

MINOR

F. Waste disposal/processing

MINOR

G. Termination

MINOR

$\mathrm{H}$. Induced technology

UNASSESSED

Example Impacts: 1. A, B, C, D, F, G. Sport species may be driven from a traditional hunting or fishing area.

Data Available:

Models Available: 
Technology: $\quad 1.3 .2$

Geopower

Geothermal Energy

Residual: $\quad 2.4$

Biological residuals

Recipient: 3.3 .3

Social-Cultural domain

Aesthetics

Potential Environmental Impact: A. Exploration

MINOR

B. Construction

SEVVERE

C. Exploitation

SEVERE

D. Transformation

MINOR

E. Transport

MODERATE

F. Waste disposal/processing

SE VERE

G. Termination

MODERATE

$\mathrm{H}$. Induced technology

UNASSESSED

Example Impacts: 1. A, B, C, D, E, F. Loss of vegetation may denude aesthetically appealing terrain.

Data Available:

Models Available: 
Technology: $\quad 1.3 .2$

Geopower

Geothermal Energy

Residual: $\quad 2.4$

Biological residuals

Recipient: 3.3 .4

Social-Cultural domain

Quality of life

Potential Environmental Impact: A. Exploration

MINOR

B. Construction

MODERATE

C. : Exploitation

MODERATE

D. Transformation

MODERATE

E. Transport

MINOR

F. Waste disposal/processing

MODERATE

G. Termination

MINOR

H. Induced technology

UNASSESSED

Example Impacts: 1. A, B, C, D, E, F. Most geothermal resources are in regions of natural beauty and fragile ecology, high potential for impact on quality of life.

Data Available:

Models Available: 
Technology: $\quad 1.3 .2$

Geopower

Geothermal Energy

Residual: $\quad 2.4$

Biological residuals

Recipient: 3.3.4.1

Social-Cultural domain

Quality of life

Health and safety

Potential Environmental Impaci: A. Exploration

NONE

B. Construction

NONE

C. Exīplo1tatiun

MINOR

D. Transformation

NONE

E. Transport

NONE

F. Waste disposal/processing

MINOR

G. Termination

NONE

H. Induced technology

NONE

Example Impacts: 1. C, F. Killing of vegetation may create fire hazard.

Data Available:

Models Available: 
Technology: $\quad 1.3 .2$

Geopower

Geothermal Energy

Residual: 2.4

Biological residuals

Recipient: $\quad 3.3 .4 .2$

Social-Cultural,domain

Quality of life

Ltfe style

Potential Environmental Impact: A. Exploration

MINOR

B. Construction

MODERATE

C. Exploitation

MODERATE

D. Transformation

MODERATE

E. Transport

MINOR

F. Waste disposal/processing

MODERATE

G. Termination

MINOR

$\mathrm{H}$. Induced technology

UNASSESSED

. Example Impacts: 1. A, B, C, D, E, F. Loss of vegetation, grazing and browse may may adversely affect adjacent Indian communities.

Data Available:

Models Available: 


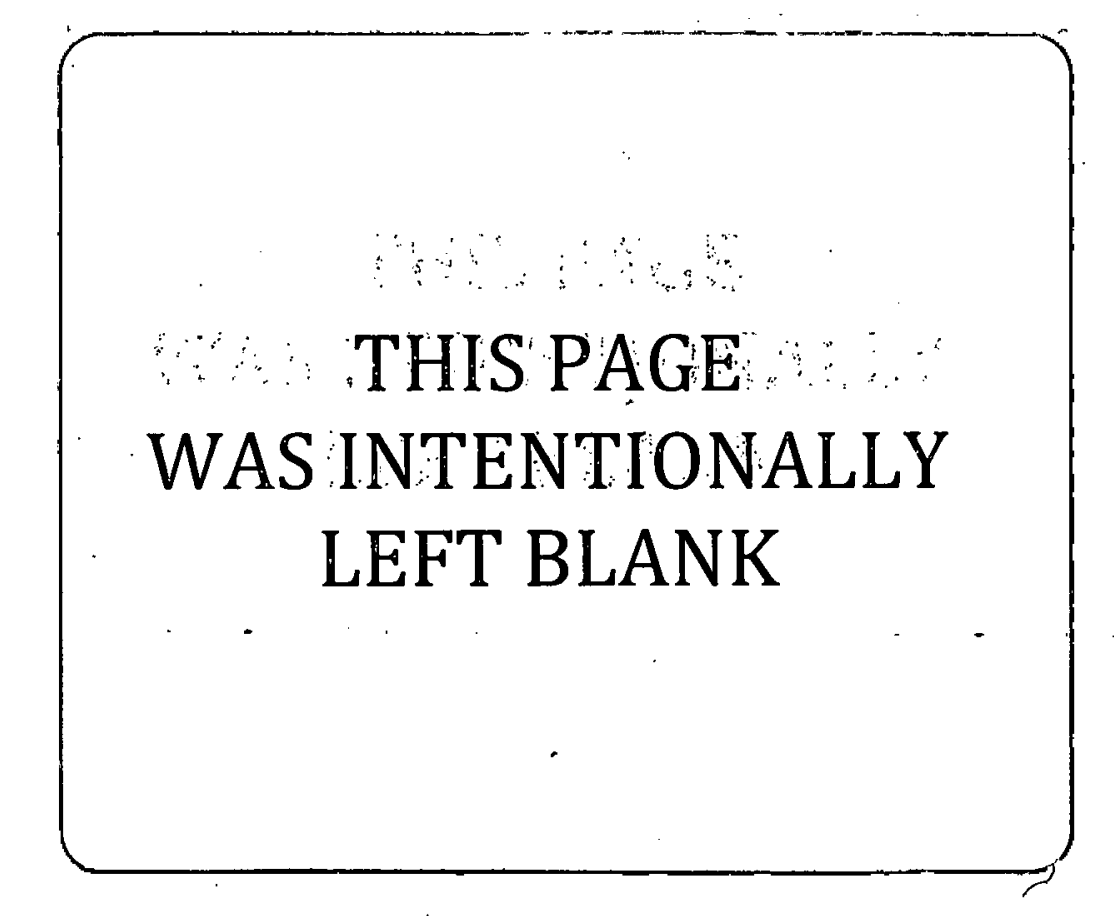

Blank

$$
B-108
$$


I

$D$

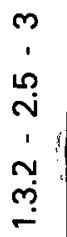


Technology: $\quad 1.3 .2$

Geopower

Geothermal Energy

Residual: $\quad 2.5$

Social-Cultural residuals

Recipient: $\quad 3.1$

Physical and chemical domain

Potential Environmental Impact: A. Exploration

MINOR

B. Construction

SEVERE

C. Exploitation

SE VERE

D. Transformation

MODERATE

E. Transport

MINOR

F. Waste disposal/processing

SE VERE

G. Termination

MINOR

H. Induced technology

UNASSESSED

Example.Impacts: 1. B. Alteration in land use limits recovery of other resources.

2. C. Alteration in land use affects soil.

Data Available: 1. NAWDEX

2. ENDEX

3. AEROS

4. ORRMIS

Models Available: SUWQI 
Technology: $\quad 1.3 .2$

Geopower

Geothermal Energy

Residual: $\quad 2.5$

Social-Cultural residuals

Recipient: 3.1 .1

Physical and chemical domain

Earth

Potential Environmental Impact: A. Exploration

MINOR

B. Construction

SEVERE

C. Exploitation

SE VERE

D. Transformation

MODERA TE

E. Transport

MINOR

F. Waste disposal/processing

SEVERE

G. Termination

MINOR

$\mathrm{H}$. Induced technology

UNASSESSED

Example Impacts: 1. B. Alteration in land use limits recovery of other resources.

2. C. Alteration in land use required change to topography (grading).

Data Available:

ORRMIS

Models Available: 
Technology: $\quad 1.3 .2$

Geopower

$$
\text { Geothermal Energy }
$$

Residual: $\quad 2.5$

Social-Cultural residuals

Recipient: 3.1.1.1

Physical and chemical domain

Earth

Topography

Potential Environmental Impact: A. Exploration

MINOR

B. Construction

SE VERE

C. Exploitation

MODERATE

D. Transformation

MODERATE

E. Transport

MINOR

F. Waste disposal/processing

SEVERE

G. Termination

MINOR

H. Induced technology

UNASSESSED

Example Impacts: 1. A, B, C, D, E, F, G. Alteration in land use requires change to topography (grading).

Data Available: ORRMIS

Models Available: 
Technology: $\quad 1.3 .2$

Geopower

Geothermal Energy

Residual: $\quad 2.5$

Social-Cultural residuals

Recipient: 3.1 .1 .2

Physical and chemical domain

Earth

Résources

Potential Environmental Impac's: A. Exploration

MINOR

B. Construction

SE VERE

C. Exploitation

MODERATE

D. Transformation

MODERATE

E. Transport

MINOR

F. Waste disposal/processing

MODERA TE

G. Termination

MINOR

$H$. Induced technology

UNASSESSED

Example Impacts: 1. A, B, C, D, E, F, G. Alteration in land use impacts other resource recovery efforts.

Data Available: $\quad$ ORRMIS

Models Available:

B -112 
Téchnology: $\quad 1.3 .2$

Geopower

Geothermal Energy

Residual: $\quad 2.5$

Social-Cultural residuals

Recipient: 3.1 .1 .3

Physical and chemical domain

Eərth

Slow processes

Potential Environmental Impacti: A. Exploration

NONE

B. Construction

MODERATE

C. Exploitation

MODERATE

D. Transformation

MODERATE

E. Transport

MINOR

F. Waste disposal/processing

MODERATE

G. Termination

MINOR

H. Induced technology

UNASSESSED

Example Impacts: 1. A, B, C, D, E, F, G. Altered land use causing increased traffic over area resulting in compaction.

Data Available:

Models Available: 
Technology: $\quad 1.3 .2$

Geopower

\section{Geothermal Energy}

Residual: 2.5

Social-Cultural residuals

Recipient: 3.1 .1 .3 .5

Physical and chemical domain

Earth

$$
\begin{aligned}
& \text { Slow processes } \\
& \text { Compaction }
\end{aligned}
$$

Potential Environmental Impacs: A. Exploration

NONE

B. Construction

MODERATE

C. Exploitation

MODERATE

D. Transformation

MODERATE

E. Transport

MINOR

F. Waste disposal/processing

MODERATE

G. Termination

MINOR

H. Induced technology

UNASSESSED

Example Impacts: 1. A, B, C, D, E, F, G. Altered land use causing increased traffic over area resulting in compaction.

Data Available:

Models Available: 
Technology: 1.3 .2

Geopower

Geothermal Energy

Residual: $\quad 2.5$

Social-Cultural residuals

Recipient: 3.1 .1 .4

Physical and chemical domain

Earth

Rapid processes

Potential Environmental Impact: A. Exploration

NONE

B. Construction

MODERATE

C. Exploitation

MODERATE

D. Transformation

MINOR

E. Transport

MINOR

F. Waste disposal/processing

MINOR

G. Termination

MINOR

H. Induced technology

UNASSESSED

Example Impacts: ${ }^{1}$ B, C, F. Altered land use may result in denudation and promote erosion.

Data Available:

Models Available: 
Technology: $\quad 1.3 .2$

Geopower

Geothermal Energy

Residual: $\quad 2.5$

Social-Cultural residuals

Recipient: 3.1.1.4.1

Physical and chemical domain

Earth

Rápid processes

Flood

Potential Environmental Impač: A. Exploration

NONE

B. Construction

MINOR

C. Exploitation

MINOR

D. Transformation

MINOR

E. Transport

NONE

F. Waste disposal/processing

MINOR

G. Termination

MINOR

H. Induced technology

UNASSESSED

Example Impacts: 1. B, C, D, F, G. Altered land use may make area more susceptible to flooding:

Data Available:

Models Available: 
Technology: $\quad 1.3 .2$

Geopower

Geothermal Energy

Residual: 2.5

Social-Cultural residuals

Recipient: $\quad 3.1 .1 .4 .2$

Physical and chemical domain

Earth

$$
\begin{gathered}
\text { Rapid processes } \\
\text { Erosion }
\end{gathered}
$$

Potential Environmental Impact: A. Exploration

NONE

B. Construction

MODERATE

C. Exploitation

MODERATE

D. Transformation

MINOR

E. Transport

MINOR

F. Waste disposal/processing

MINOR

G. Termination

MINOR

H. Induced technology

UNASSESSED

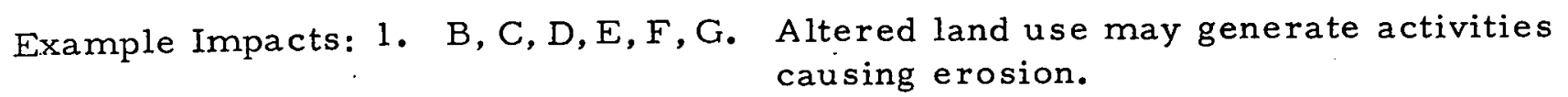

Data Available:

Models Available: 
Technology: $\quad 1.3 .2$

Geopower

Geothermal Energy

Residual: $\quad 2.5$

Social-Cultural residuals

Recipient: 3.1.1.4.6

Physical and chemical domain

Earth

Rapid processes

Air movement

Potential Environmental Impact: A. Exploration NONE

B. Construction NONE

C. Exploitation MINOR

D. Transformation MINOR

E. Transport MINOR

F. Waste disposal/processing NONE

G. Termination : NONE

H. Induced technology NONE

Example Impacts: 1. C, D, E. Alteration of land use may cause change in wind patterns.

Data Available:

Models Available: 
Technology: $\quad 1.3 .2$

Geopower

Geothermal Energy

Residual: $\quad 2.5$

Social-Cultural residuals

Recipient: 3.1 .1 .5

Physical and chemical domain

Earth

Unique features

Potential Environmental Impact: A. Exploration

NONE

B. Construction

MODERATE

C. Exploitation

SE VERE

D. Transformation

MODERATE

E. Transport

MINOR

F. Waste disposal/processing

MODERATE .

G. Termination

MINOR

$\mathrm{H}$. Induced technology

NONE

Example Impacts: 1. B, C, D, E, F, G. Altered land use may harm a unique feature of the area such as aquifer recharge.

Data Available:

Models Available: 
Technology: $\quad 1.3 .2$

Geopower

Geothermal Energy

Residual: . 2.5

Social-Cultural residuals

Recipient: 3.1 .1 .5 .1

Physical and chemical domain

Earth

Unique features

Aquifer recharge

Potential Environmental Impact: A. Exploration

NONE

B. Construction

MODERATE

C. Exploitation

SE VERE

D. Transformation

MODERATE

E. Transport

MINOR

F. Waste disposal/processing

MODERATE

G. Termination

MINOR

H. Induced technology

NONE

Example Impacts: 1. B, C, D, E, F, G. Altered land use may harm a unique feature of the area such as aquifer recharge.

Data Available:

Models Available: 
Technology: $\quad 1.3 .2$

Geopower

Geothermal Energy

Residual: $\quad 2.5$

Social-Cultural residuals

Recipient: 3.2

Biological domain

Potential Environmental Impacť: A. Exploration

MINOR

B. Construction

MODERATE

C. Exploitation

MODERATE

D. Transformation

NONE .

E. Transport

MINOR

F. Waste disposal/processing

MODERATE

G. Termination

MINOR

$\mathrm{H}$. Induced technology

UNASSESSED

Example Impacts: $\quad$ 1. A, B, C, E, F, G. Alteration in land use may result in reduction in numbers of wild and domestic mammals, also fish and birds.

Data Available:

NAW DEX

Models Available: 
Technology: $\quad 1.3 .2$

Geopower.

Geothermal Energy

Residual: 2.5

Social-Cultural residuals

Recipient: 3.2 .1

Biological domain

Animals

Potential Environmental Impac's: A. Exploration

NONE

B. Construction

MODERATE

C. Exploitation

MODERATE

D. Transformation

NONE

E. Transport

MINOR

F. Waste disposal/processing

MODERATE

G. Termination

MINOR

H. Induced technology

UNASSESSED

Example Impacts: 1. A, B, C, E, F, G. Alteration in land use may result in reduction in numbers of wild and domestic mammals, also fish and birds.

Data Available:

NAWDEX

Modcls $\Lambda$ vailable: 
Technology: $\quad 1.3 .2$

Geopower

Geothermal Energy

Residual: $\quad 2.5$

Social-Cultural residuals

Recipient: $\quad 3.2 .2$

Biological domain

Plants

Potential Environmental Impact: A. Exploration

MINOR

B. Construction

MODERATE

C. Exploitation MODERATE

D. Transformation

NONE

E. Transport

MINOR

F. Waste disposal/processing

MODERATE

G. Termination

MINOR

$\mathrm{H}$. Induced technology

UNASSESSED

Example Impacts: 1. A, B, C, E, F, G. Alteration in land use may result in changes in numbers and kind of trees, grasses, shrubs.

Data Available: . NAWDEX

Models Available: 
Technology: $\quad 1.3 .2$

Geopower

Geothermal Energy

Residual: $\quad 2.5$

Social-Cultural residuals

Recipient: $\quad 3.3$

Social-Cultural domain

Potential Environmental Impact: A. Exploration

B. Construction

SEVERE

C. Exploitation

SEVERE

D. Transformation

SE VERE

E. Transport

SEVERE

F. Waste disposal/processing

SEVERE

G. Termination

SE VERE

H. Induced technology

SE VERE

UNASSESSED

Example Impacts: 1. A, B, C, D, E, F, G. Serious constraint on geothermal development imposed by sociocultural ambient may be the meeting of legal requirements.

Data Available: 1. EMDB

2. NAWDEX

3. DOI/DF -73-001

4. PL91-581

5. ERDA/EA/TVL

6. ORRMIS

7. IVEP

8. DOI/EIS. 1

9. DOI/EIS 2

10. DOI/EIS 4

Models Available: 1. OSSIM

2. ORRMIS 
Technology:

$$
\begin{aligned}
& \text { 1.3.2 } \\
& \text { Geopower } \\
& \text { Geothermal Energy }
\end{aligned}
$$

Residual: $\quad 2.5$

Social-Cultural residuals

Recipient: 3.3 .1

Social-Cultural domain

Land use

Potential Environmental Impac's: A. Exploration

SEVERE

B. Construction

SE VERE

C. Exploitation

SEVERE

D. Transformation

MODERATE

E. Transport

MODERATE

F. Waste disposal/processing

SEVERE

G. Termination

MODERA TE

$\mathrm{H}$. Induced technology

UNASSESSED

Example Impacts: 1. A, B, C, D, E, F, G. All phases of land use affected by its dedication to geothermal development.

Data Available: 1. EMDḄ

2. DOI/DF -73-001

3. ORRMIS

Models Available: 1. OSSIM

2. ORRMIS 
Technology: $\quad 1.3 .2$

Geopower

Geothermal Energy

Residual: $\quad 2.5$

Social-Cultural residuals

Recipient: $\quad 3.3 .2$

Social-Cultural domain

Recreation

Potential Environmental Impact: A. Exploration SEVERE

B. Construction SEVERE

C. Exploitation SEVERE

D. Transformation SEVERE

E. Transport SEVERE

F, Waste disposal/processing SEVERE

G. Termination SEVERE

$\mathrm{H}$. Induced technology UNASSESSED

Example Impacts: 1. A, B, C, D, E, F, G. Recreational activities - hunting, fishing, hiking - curtailed by dedication of land to geothermal development.

Data Available: 1. NAWDEX

2. $\mathrm{DOI} / \mathrm{DF}-73-001$

Models Available: 
Technology: 1.3 .2

Geopower

Geothermal Energy

Residual: $\quad 2.5$

Social-Cultural residuals

Recipient: 3.3 .3

Social-Cultural domain

Aesthetics

Potential Environmental Impact: A. Exploration

SEVERE

B. Construction

SE VERE

C. Exploitation

SE VERE

D. Transformation

SE VERE

E. Transport

SE VERE

F. Waste disposal/processing

SE VERE

G. Termination

SE VERE

H. Induced technology

UNASSESSED

Example Impacts: 1. E. Electric power transmission lines may be unattractive aesthetically within certain cultural contexts.

Data Available:

Models Available: 
Technology: $\quad 1.3 .2$

Geopower

Geothermal Energy

Residual: $\quad 2.5$

Social-Cultural residuals.

Recipient: 3.3 .4

Social-Cultural domain

Quality of life

Potential Environmental Impact: A. Exploration

MODERATE

B. Construction

SE VERE

C. Exploitation

SE VERE

D. Transformation

MODERATE

E. Transport

MODERATE

F. Waste disposal/processing

SE VERE

G. Termination

SEVERE

H. Induced technology

UNASSESSED

Example Impacts: A, B, C, D, E, F, G. Most geothermal resource areas in thinly populated regions, some with distinctive but vulnerable cultures.

Data Available:

IVEP

Models Available: 
Technology: 1.3 .2

Geopower

Geothermal Energy

Residual: $\quad 2.5$

Social-Cultural residuals

Recipient: 3.3.4.1

Social-Cultural domain

Quality of life

Health and safety

Potential Environmental Impací: A. Exploration

MINOR

B. Construction

MODERATE

C. Exploitation

SEVERE

D. Transformation

MODERATE

E. Transport

MINOR

F. Waste disposal/processing

SEVERE

G. Termination

MINOR

H. Induced technology

UNASSESSED

Example Impacts: 1. A, B, C. Noise levels may be objectionable within certain cultural contexts.

Data Available: IVEP

Models Available:

B -129 
Tëchnölogy: -1.3 .2$

-...... $=$ Geopower

Geothermal Energy

Residual: $\quad 2.5$

Social-Cultural residuals

Recipient: $\quad 3.3 .4 .2$

Social-Cultural domain

Quality of life

Life style

Potential Environmental Impact: A. Exploration

MODERATE

B. Construction

SEVERE

C. Exploitation

SEVERE

D. Transformation

MODERATE

E. Transport

MODERATE

F. Waste disposal/processing

MODERATE

$G$. Termination

SEVERE

H. Induced technology

UNASSESSED

Example Impacts: 1. A, B, C, E. Employment of local Indian labor affects life style through income.

2. G. Termination may have adverse effect on life style through loss of income.

Data Available:

Models Available: 
Technology: $\quad 1.3 .2$

Geopower

Geothermal Energy

Residual: $\quad 2.5$

Social-Cultural residuals

Recipient: $\quad 3.3 .4 .3$

Social-Cultural domain

Quality of life

Population characteristics

Potential Environmental Impact: A. Exploration

MINOR

B. Construction

SEV ERE

C. Exploitation

SEVERE

D. Transformation

MINOR

E. Transport

MINOR

F. Waste disposal/processing

SEVERE

G. Termination

SEVERE

H. Induced technology

UNASSESSED

Example Impacts: 1. B, C. Employment of transient foreign labor affects population ethnic background.

2. B, C. Loss (or gain) of tourist attractions changes population mix.

Data Available: IVEP

Models Available: 
Technology: $\quad 1.3 .2$

Geopower

Geothermal Energy

Residual: $\quad 2.5$

Social-Cultural residuals

Recipient: $\quad 3.3 .5$

Social-Cultural domain

Legal aspects

Potential Environmental Impact:
A. Exploration
SEVERE
B. Construction
SEVERE
C. Exploitation
SEVERE
D. Transformation
SEVERE
E. Transport
MODERATE
F. Waste disposal/processing
SEVERE
G. Termination
SEVERE
H. Induced technology
UNASSESSED

Example Impacts: 1. A, B, E, F. Acquisition of land titles and rights of way affect interests of residents.

2. A, B. Public access to resource development area may require adjudication.

Data Available: 1. ERDA/EA/TVL

2. DOI/EIS 1

3. DOI/EIS 2

4. DOI/EIS 4

Models Availablc: 
Technology: $\quad 1.3 .2$

Geopower

Geothermal Energy

Residual: $\quad 2.5$

Social-Cultural residuals

Recipient: $\quad 3.4$

Economic domain

Potential Environmental Impac's: A. Exploration

MINOR

B. Construction

SEVERE

C. Exploitation

SEVERE

D. Transformation

MODERATE

E: Transport

MODERATE

F. Waste disposal/processing MODERATE

G. Termination

SEVERE

$H$. Induced technology

UNASSESSED

Example.Impacts: 1. G. Termination reduces community tax base, curtails public services.

Data Available: IVEP

Models Available: 
Technology: 1.3 .2

Geopower

Geothermal Energy

Residual: $\quad 2.5$

Social-Cultural residuals

Recipient: 3.4 .1

Economic domain

Demographic factors

Potential Environmental Impact: A. Exploration

MINOR

B. Construction

SEVERE

C. Exploitation

SEVERE

D. Transformation

MODERATE

E. Transport

MODERATE

F. Waste disposal/processing

MODERATE

G. Termination

SEVERE

H. Induced technology

UNASSESSED

Example Impacts: 1. B,C. Hiring relatively large work force affects thinly populated communities.

2. B, C. Increased demand for consumer goods affects thinly populated communities.

Data Available: IVEP

Models Available: 
Technology: $\quad 1.3 .2$

Geopower

Geothermal Energy

Residual: $\quad 2.5$

Social-Cultural residuals

Recipient: $\quad 3.4 .2$

Economic domain

Infrastructure domain

Potential Environmental Impact: A. Exploration

MINOR

B. Construction

MODERATE

C. Exploitation

MODERATE

D. Transformation

MODERATE

E. Transport

MINOR

F. Waste disposal/processing

MODERATE

G. Termination

SEV ERE

H. Induced technology

UNASSESSED

Example Impacts: 1. B,C. Employees' families tax educational system. 2. B,C. New employees tax public services.

Data Available:

Models Available:

$\mathrm{B}-135$ 
Blank

B. 136 
I

in

i

ஸุ

$m$

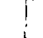

D 
Technology: $\quad 1.3 .2$

Geopower

Geothermal Energy

Residual: 2.6

Economic residuals

Recipient: 3.1

Physical and chemical domain

Potential Environmental Impaç: A. Exploration

MODERATE

B. Construction

SEVERE

C. Exploitation

SEVERE

D. Transformation

MINOR

E. Transport

MODERATE

F. Waste disposal/processing

SEVERE

G. Termination

SEVERE

H. Induced technology

UNASSESSED

Example Impacts: 1. A, B, C, D, E, F. Construction and operating personnel may pollute local water supplies.

Data Available: 1. CRIB

2. $\mathrm{COM} / \mathrm{DF}-73-022$

Models Available: 1. SRIEM

2. EEPM

3. WISE

4. OSSIM

5. SUWQI

6. GEOCOST 
Technology: $\quad 1.3 .2$

Geopower

Geothermal Energy

Residual: $\quad 2.6$

Economic residuals

Recipient: 3.1 .1

Physical and chemical domain

Earth

Potential Environmental Impact: A. Exploration

B. Construction

C. Exploitation

D. Transformation

E. Transport

NONE

MODERATE

MODERATE

NONE

F. Waste disposal/processing

NONE

G. Termination

NONE

H. Induced technology

NONE

UNASSESSED

Example Impacts: 1. B, C. Housing construction consumes resources.

Data Available: 1. CRIB

2. $\mathrm{COM} / \mathrm{DF}-73-022$

Models Available: 1. SRIEM

2. GEOCOST 
Technology: $\quad 1.3 .2$

Geopower

Geothermal Energy

Residual: $\quad 2.6$

Economic residuals

Recipient: $\quad 3.1 .1 .2$

Physical and chemical domain

Earth

Resources

Potential Environmental Impací: A. Exploration

NONE

B. Construction

MODERATE

C. Exploitation MODERATE

D. Transformation NONE

E. Transport

NONE

F. Waste disposal/processing

NONE

G. Termination NONE

$\mathrm{H}$. Induced technology

UNASSESSED

Example Impacts: 1. B, C. Housing construction consumes resources.

Data Available: 1. CRIB

2. $\mathrm{COM} / \mathrm{DF}-73-022$

Models Available: 1. SRIEM

2. GEOCOST 
Technology: $\quad 1.3 .2$

Geopower

$$
\text { Geothermal Energy }
$$

Residual: 2.6

Economic residuals

Recipient: 3.1.2

Physical and chemical domain

Water

Potential Environmental Impac'c:
A. Exploration
MODERATE
B. Construction
SEVERE
C. Exploitation
SEVERE
D. Transformation
MINOR
E. Transport
MODERATE
F. Waste disposal/processing
SEV ERE
G. Termination
SEVERE
$\mathrm{H}$. Induced technology
UNASS ESSED

Example Impacts:1. A, B, C, D, E, F. Construction and operating personnel may pollute local water supplies.

Data Available:

Models Availablc:l. SUWQI

2. GEOCOST 
Technology: 1.3 .2

Geopower

Geothermal Energy

Residual: 2.6

Economic residuals

Recipient: 3.1 .3

Physical and chemical domain

Air

Potential Environmental Impact: A. Exploration

MINOR

B. Construction

MODERATE

C. Exploitation

MODERATE

D. Transformation

MINOR

E. Transport

MODERATE

F. Waste disposal/processing

MODERATE

G. Termination

MODERATE

$\mathrm{H}$. Induced technology

UNASSESSED

Example Impacts:1. A, B, C, D, E, F. Construction and operating personnel may contribute to air pollution.

Data Available: $\quad$ COM/DF $-73-022$

Models Available:1. OSSIM

2. EEPM

3. WISE 
Technology: $\quad 1.3 .2$

Geopower

Geothermal Energy

Residual: 2.6

Economic residuals

Recipient: 3.2

Biological domain

Potential Environmental Impací: A. Exploration

MINOR

B. Construction

SEVERE

C. Exploitation

SEVERE

D. Transformation

MODERATE

E. Transport

MODERATE

F. Waste disposal/processing

MODERATE

G. Termination

MODERATE

H. Induced technology

UNASSESSED

Example Impacts:1. A, B, C, D, E, F. Increased work force, housing and business activity perturb animal population.

Data Available:

Models Available: 
Technology: $\quad 1.3 .2$

Geopower

Geothermal Energy

Residual: $\quad 2.6$

Economic residuals

Recipient: 3.2.1

Biological domain

Animals

Potential Environmental Impact: A. Exploration

B. Construction

MINOR

C. Exploitation

SEV ERE

D. Transformation

SEV ERE

E. Transport

MODERATE

F. Waste disposal/processing

MODERATE

G. Termination

MODERATE

H. Induced technology

MODERATE

UNASSESSED

Example Impacts:1. A, B, C, D, E, F. Inc reased work force, housing and business activity perturb animal population.

Data Available:

Models Available: 
Technology: 1.3 .2

Geopower

Geothermal Energy.

Residual: 2.6

Economic residuals.

Recipient: 3.2 .2

Biological domain

Plants

Potential Environmental Impact: A. Exploration

MINOR

B. Construction

SEVERE

C. Exploitation

SEVERE

D. Transformation

MODERATE

E. Transport

MODERATE

F. Waste disposal/processing

MODERATE

G. Termination

MODERATE

$\mathrm{H}$. Induced technology

UNASSESSED

Example Impacts:1. A, B, C, D, E, F. Increased work force, housing, and business activity damage vegetation.

Data Available:

Models Available: 
Technology: 1.3 .2

Geopower

Geothermal Energy

Residual: 2.6

Economic residuals

Recipient: 3.3

Social-Cultural domain

Potential Environmental Impact: A. Exploration

MINOR

B. Construction

SEVERE

C. Exploitation

SEVERE

D. Transformation

MODERATE

E. Transport

MODERATE

F. Waste disposal/processing

G. Termination

MODERATE

$H$. Induced technology

SEVERE

UNASSESSED

Example Impacts: 1. A, B, C, D, E, F. Housing requirements make demands on land use for residential purpose.

Data Available: 1. OBERS

2. IVEP

3. BEIC

4. ERDA-2837

5. SEN-93-GR

6. HOUSE - $93-29$

7. ERDA-2821

Models Available: 1. SRIEM

2. BESOM

3. OSSIM

4. EAM/CIM

5. DRI

6. UH/INDUSTRY

7. ESMRMR

8. ULUPF

9. FPCBOP8 
Technology: $\quad 1.3 .2$

Geopower

Geothermal Energy

Residual: 2.6

Economic residuals

Recipient: 3:3.1

Social-Cultural domain

Land use

Potential Environmental Impact: A. Exploration

MINOR

B. Construction

SEV ER E

C. Exploitation

SEVERE

D. Transformation

MINOR

E. Transport

MINOR

F. Waste disposal/processing

MINOR

G. Termination

SEV F.R F.

H. Induced technology

UNASSESSED

Example Impacts: $\quad$ 1. A, B, C, D, E, F. Housing requirements make demands on land use for residential purpose.

Data Available:

Models Available: 1. OSSIM

2. SRIEM

3. BESOM

4. UंLUPF 


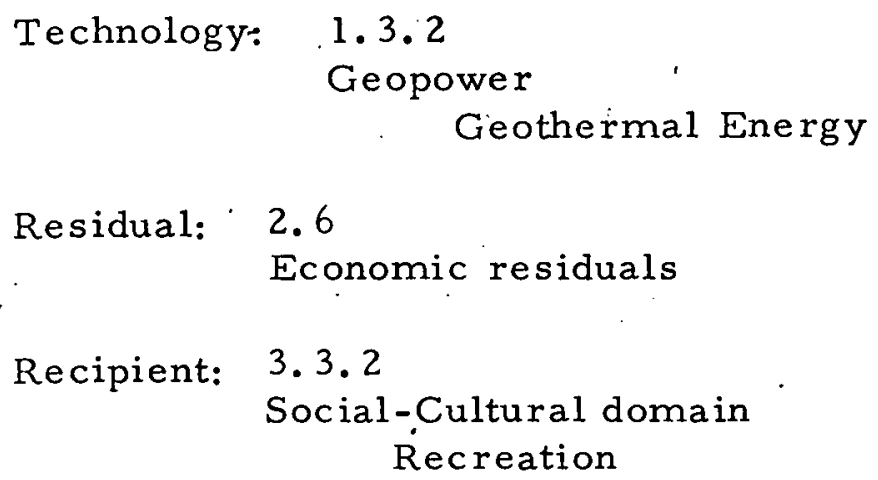

Potential Environmental Impac's: A. Exploration

MINOR

B. Construction

SEVERE

C. Exploitation SEVERE

D. Transformation MINOR

E. Transport

MINOR

F. Waste disposal/processing

MINOR

G. Termination

$\mathrm{H}$. Induced technology

UNASSESSED

Example Impacts: $\quad$ 1. A, B, C, D, E, F. Personnel and dependents make demands on recreational facilities.

Data Available:

Models Available: 
Technology: $\quad 1.3 .2$

Geopower

Geothermal Energy

Residual: $\quad 2.6$

Economic residuals

Recipient: 3.3 .3

Social-Cultural domain

Aesthetics

Potential Environmental Impac's:

A. Exploration

B. Construction

C. Exploitation

D. Transformation

E. Transport

F. Waste disposal/processing

G. Termination

H. Induced technology
MINOR

SEV ERE

SEVERE

MODERATE

MINOR

MINOR

MODERATE

UNASS ESSED

Example Impacts: $\quad$ 1. B, C, D, E, F. Housing camp appearance may lower aesthetic values of wild area.

Data Available:

Models Available: 
$\begin{aligned} \text { Technology: } & \begin{array}{l}1.3 .2 \\ \text { Geopower } \\ \text { Geothermal Energy }\end{array}\end{aligned}$

Residual: 2.6

Economic residuals

Recipient: 3.3.4

Social-Cultural domain

Quality of life

Potential Environmental Impacı́: A. Exploration

MODERATE

B. Construction

SEVERE

C. Exploitation

SEVERE

D. Transformation

MODERATE

E. Transport MODERATE

F. Waste disposal/processing MODERATE

G. Termination SEVERE

H. Induced technology UNASSESSED

Example Impacts: 1. G. End of project may create hardship to local serviceoriented business.

Data Available: $\quad$ 1. OBERS

2. IVEP

Models Available:

SRIEM 
Technology: $\quad 1.3 .2$

Geopower

Geothermal Energy

Residual: 2.6

Economic residuals

Recipient: 3.3 .4 .1

Social-Cultural domain

Quality of life

Hearth and safety

Potential Environmental Impact: A. Exploration

MINOR

B. Construction

MODERATE

C. Exploitation

MODERAT E

D. Transformation

MODERATE

E. Transport

MODERATE

F. Waste disposal/processing

MODERATE

G. Termination

MODERATE

$H$. Induced technology

UNASSESSED

Example Impacts: $\quad$ 1. A, B, C, D, E, F. Living conditions in wilderness area may expose personnel and dependents to safety hazards.

Data Available:

Models Available: 
Technology: 1.3 .2

Geopower

Geothermal Energy

Residual: $\quad 2.6$

Economic residuals

Recipient: 3.3.4.2

Social-Cultural domain

Quality of lifc

Life- style

Potential Environmental Impact
A. Exploration
MINOR
B. Construction
MODERATE
C. Exploitation
SEVERE
D. Transformation
MODERATE
E. Transport
MINOR
F. Waste disposal/processing
MODERATE
G. Termination
SEVERE
H. Induced technology
UNASSESSED

Example Impacts: 1. A, B, C, D, E, F. Added payroll may create goods shortages for local population.

Data Available:

Models Available: 
Technology: $\quad 1.3 .2$

Geopower

Geothermal Energy

Residual: 2.6

Economic residuals

Recipient: 3.3 .4 .3

Social-Cultural domain

Quality of life

Population characteristics

Potential Environmental Impact: A. Exploration

MODERATE

B. Construction

SEVERE

C. Exploitation

SEVERE

D. Transformation

MODERATE

E. Transport

MODERATE

F. Waste disposal/processing

MODERATE

G. Termination

SEVERE

H. Induced technology

UNASSESSED

$\begin{aligned} \text { Example Impacts: } \quad \text { 1. A, B, C, D, E, F. } & \begin{array}{l}\text { Work force may have different age, } \\ \text { educational, ethnic background from } \\ \text { local population. }\end{array}\end{aligned}$

Data Available:

1. OBERS

2. IVEP

Models Available:

SRIEM 
Technology: $\quad 1.3 .2$

Geopower

Geothermal Energy

Residual: 2.6

Economic residuals

Recipient: 3.3 .5

Social-Cultural domain

Legal aspects

Potential Environmental Impací: A. Exploration

MINOR

B. Construction

MINOR

C. Exploitation

MINOR

D. Transformation

MINOR

E. . Transport

MINOR

F. Waste disposal/processing

MINOR

G. Termination

MINOR

H. Induced technology

UNASSESSED

Example Impacts: $\quad$ 1. A, B, C, D, E, F. Personnel and associated business may encounter zoning problems.

Data Available:

1. IV EP

2. BEIC

3. ERDA-2837

4. $\mathrm{SEN}-93-\mathrm{GR}$

5. HOUSE $-93-29$

6. ERDA-2821

Models Available: 1. SRIEM

2. BESOM

3. EAM/CIM

1. DRI

5. UH/INDUSTRY

6. ESMRMR

7. FPC BOP8 
Technology: $\quad 1.3 .2$

Geopower

Geothermal Energy

Residual: 2.6

Economic residuals

Recipient: 3.4

Economic domain

Potential Environmental Impact: A. Exploration

MODERATE

B. Construction

SEVERE

C. Exploitation

SEVERE

D. Transformation

MODERATE

E. Transport

MODERATE

F. Waste disposal/processing

SEV ERE

$G$. 'Iermination

SEVERE

$\mathrm{H}$. Induced technology

UNASSESSED

Example Impacts: $\quad$ l. A, B, C, D, E, F. Personnel contribute to local tax base.

Data Available: 1. OBERS

2. IVEP

3. BEIC

4. DOI/BLM

5. COM/DF-73-022

6. DOCBOC 11

7. ORRMIS

8. RUSTIC

9. PANS

10. DOCBOC 10

\begin{abstract}
Models Available:
\end{abstract}
1. OSSIM
2. PIES
3. SRIEM
4. BESOM
5. SEEDIS
6. ESPM
7. BLS/DF-73-007
8. $\mathrm{CES} / \mathrm{HNL} 2$
9. $\mathrm{CES} / \mathrm{HNL} 3$
10. LEMFEU

11. WEFM

12. PILOT

13. ETA

14. PREVIEWS

15. MIROH

16. EEDF

17. ERDM

18. WISE

19. EEPM

20. EEIED
21. UH/HOUSTON

22. UH/INDUSTRY

23. EMPP

24. EAM/CIM

25. ESMRMR

26. SDNM

27. SEAS

28. GEOCOST 
Technology: 1.3 .2

Geopower

Geothermal Energy

Residual: $2.6^{\circ}$

Economic residuals

Recipient: 3.4 .1

Economic domain

Demographic factors

Potential Environmental Impact: A. Exploration

MODERATE

B. Construction

SEVERE

C. Exploitation

SEVERE

D. Transformation

MODERATE

E. Transport

MODERATE

F: Waste disposal/processing

SEVERE

G. Termination

SEV ERE

H. Induced technology

UNASSESSED

Example Impacts: $\quad$ 1. A, B, C, D, E, F. Personnel contribute to local tax base.

Data Available: $\quad$ 1. OBERS

2. IVEP

3. ORRMIS

4. RUSTIC

5. PANS

6. DOCBOC 10

$\begin{array}{lllll}\text { Models Available: } & \text { 1. PIES } & \text { 9. CES/HNL 3 } & \text { 17. ERDM } \\ & \text { 2. SRIEM } & 10 . \text { LEMFEU } & \text { 18. WISE } \\ & \text { 3. BESOM } & 11 . \text { WEFM } & \text { 19. EEIED } \\ \text { 4. SEEDIS } & \text { 12. PILOT } & \text { 20. UH/HOUSTON } \\ \text { 5. ESPM } & \text { 13. ETA. } & \text { 21. SDNM } \\ \text { 6. OSSIM } & 14 . \text { PREVIEWS } & \text { 22. EMPP } \\ \text { 7. BLS/DF -73-007 } & 15 . \text { MIROH } & \\ \text { 8. CES/HNL 2 } & 16 . \text { EEDF }\end{array}$


Technology: $\quad 1.3 .2$

Geopower.

Geothermal Energy

Residual: 2.6

Economic residuals

Recipient: 3.4 .2

Economic domain

Infrastructure domain

Potential Environmental Impact: A. Exploration

MINOR

B. Construction

SEVERE

C. Exploitation

SEVERE

D. Transformation

MODERATE

E. Transport

MODERATE

F. Waste disposal/processing

MODERATE

G. Termination

SEVERE

H. Induced technology

UNASSESSED

Example Impacts: $\quad$ 1. A, B, C, D, E, F. Personnel and dependents may make heavy demands on limited public services.

Data Available:

1. BEIC

2. COM/DF-73-022

3. DOCBOC 11

4. ORRMIS

Models Availablc: 1. PIES

2. SRIEM

8. EEPM

3. BESOM

9. $\mathrm{EAM} / \mathrm{CIM}$

4. PILOT

10. UH/INDUSTRY

5. MIROH

11. ESMRMR

6. ERDM

12. SDNM

7. WISE

13. SEAS

14. GEOCOST 
$b$

p 
Technology: 1.3 .2

Geopower

Geothermal Energy

Residual: 2.7

Public health residuals

Recipient: 3.1

Physical and chemical domain

Potential Environmental Impact: A. Exploration

MINOR

B. Construction

SEVERE

C. Exploitation

SEVERE

D. Transformation

SEVERE

E. Transport

MINOR

F. Waste disposal/processing

SEVERE

G. Termination

SEVERE

H. Induced technology

UNASS ESSED

Example Impacts: 1. A, B, C, D, E, F. Quality of surface and ground waters degraded by effluents from all phases of project.

Data Available: $\quad$ 1. NAWDEX

2. STORET

3. NOAA/CDS

4. NOAA/LCS

Models Available: 1. SUWQI

2. UMWQI

3. IUWQI 
Technology: $\quad 1.3 .2$

Geopower

Geothermal Energy

Residual: 2.7

Public health residuals

Recipient: 3.1 .2

Physical and chemical domain

Water

Potential Environmental Impact: A. Exploration

MINOR

B. Construction

SEVERE

C. Exploitation

SEVERE

D. Transformation

MODERATE

E. Transport

MINOR

F. Waste disposal/processing

SEVERE

G. Termination

SEVERE

H. Induced technology

UNASSESSED

Example Impacts: $\quad$ 1. A, B, C, D, E, F. Quality of surface and ground waters degraded by effluents from all phases of project.

Data Available: 1. NAWDEX

2. STORET

Models Available: 1. SUWQI

2. UMWQI

3. IUWQI 
Technology: $\quad 1.3 .2$

Geopower

Geothermal Energy

Residual: 2.7

Public health residuals

Recipient: 3.1 .3

Physical and chemical domain

Air

Potential Environmental Impaci: A. Exploration

MINOR

B. Construction

NONE

C. Exploitation

SEVERE

D. Transformation

SEVERE

E. Transport

MINOR

F. Waste disposal/processing

SEVERE

G. Termination

SEVERE

H. Induced technology

UNASSESSED

Example Impacts: 1. A, C, D, F. Emissions harmful to public health scrubbed from air by precipitation.

Data Available: 1. NOAA/CDS

2. NOAA/LCS

Models Available: 


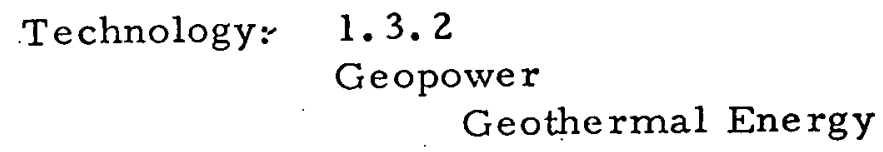

Residual: 2.7

Public health residuals

Recipient: $\quad 3.2$

Biological domain

Potential Environmental Impact: A. Exploration

SEVERE

B. Construction

SEV ERE

C. Exploitation

SEVERE

D. Transformation

MODERATE

E. Transport

MODERATE.

F. Waste disposal/processing

SEV ERE

G. Termination

MODERATE

H. Induced technology

UNASSESSED

Example Impacts: $\quad$ 1. A, B, C, E. High noise levels may interfere with nesting habits of birds.

Data Available:

NAWDEX

Models Available: 1. UMWQI

2. IUWQI 
Technology: $\quad 1.3 .2$

Geopower

Geothermal Energy

Residual: 2.7

Public health residuals

Recipient: 3.2 .1

Biological domain

Animals

Potential Environmental Impac's:
A. Exploration
B. Construction
C. Exploitation
D. Transformation
E. Transport
F. Waste disposal/processing
G. Termination
H. Induced technology

SEVERE

SEV ERE

SEVERE

MODERATE

MODERATE

SEV ERE

MODERATE

UNASSESSED

\author{
Example Impacts: $\quad$ 1.. A, B, C, F. Degradation of water quality may cause fish \\ kills. \\ 2. A, B, C, E. Noise drives away birds and mammals. \\ Data Available: \\ NAWDEX
}

Models Available: 1. UMWQI

2. IUWQI 

Technology: $\quad 1.3 .2$ Geopower
Geothermal Energy

Residual: $\quad 2.7$

Public health residuals

Recipient: 3.2 .2

Biological domain

Plants

$\begin{array}{lll}\text { Potential Environmental Impact: } & \text { A. Exploration } & \text { MODERATE } \\ & \text { B. Construction } & \text { SEVERE } \\ & \text { C. Exploitation } & \text { SEVERE } \\ & \text { D. Transformation } & \text { MODERATE } \\ & \text { E. Transport } & \text { MODERATE } \\ \text { F. Waste disposal/processing } & \text { SEVERE } \\ & \text { G. Termination } & \text { MODERATE } \\ \text { H. Induced technology } & \text { UNASSESSED }\end{array}$

Example Impacts: $\quad$ 1. A, B, C,F. Degradation of water quality may kill aquatic plants.

Data Available:

NAWDEX

Models Available: 
Technology:- $\quad 1.3 .2$

Geopower

Geothermal Energy.

Residual: 2.7

Public health residuals

Recipient: 3.3

Social-Cultural domain

Potential Environmental Impact: A. Exploration

SEV ERE

B. Construction

SEVERE

C. Exploitation

SEVERE

D. Transformation

MODERATE

E. Transport

SEVERE

F. Waste disposal/processing

SEVERE

G. Termination

MODERATE

H. Induced technology

UNASSESSED

Example Impacts: $\quad$ 1. B, C, E, F. Noise level affects quality of life for local residents.

$\begin{array}{ll}\text { Data Available: } & \text { 1. IVEP } \\ & \text { 2. EMDB } \\ & \text { 3. AEROS } \\ & \text { 4. BEIC }\end{array}$

Models Available: 1. GRAPES

2. UMWQI

3. IUWQI

4. NIH/DF-73-002 
Technology:

1.3 .2

Geopower

Geothermal Energy

Residual: 2.7

Public health residuals

Recipient: 3.3 .1

Social-Cultural domain

Land use

Potential Environmental Impacî: A. Exploration

B. Construction

SEVERE

C. Exploitation SEVERE

D. Transformation SEV ERE

E. Transport MODERATE

F. Waste disposal/processing MINOR

G. Termination SEVERE

H. Induced technology MODERATE UNASS ESSED

Example Impacts: 1. A, B, C,D,E, F. Water quality (Boron content) affects land use requirement for irrigation water.

Data Available:

Models Available: 
Technology: $\quad 1.3 .2$

Geopower

Geothermal Energy

Residual: $\quad 2.7$

Public health residuals

Recipient: 3.3 .2

Social-Cultural domain

Recreation

Potential Environmental Impact: A. Exploration

SEVERE

B. Construction

SEVERE

C. Exploitation

SEVERE

D. Transformation

MODERATE

E. Transport

MINOR

F. Waste disposal/processing

SEVERE

G. Termination

MODERATE

H. Induced technology

UNASSESSED

Example Impacts: 1. A, B, C, D, E, F. Air, water quality affect use of outdoors for camping, picnicking.

Data Available:

Models Available: 
Technology: $\quad 1.3 .2$

Geopower

Geothermal Energy

Residual: 2.7

Public health residuals

Recipient: 3.3 .3

Social-Cultural domain

Aesthetics

Potential Environmental Impact: A. Exploration

NONE

B. Construction

MODERATE

C. Exploitation

MODERATE

D. Transformation

MODERATE

E. Transport

MODERATE

F. Waste disposal/processing

MINOR

G. Termination

MINOR

H. Induced technology

UNASSESSED

Example Impacts: $\quad$ 1. C, D, E, F. Noise level affects enjoyment of open space, scenery.

Data Available:

Models Available: 
Technology: . 1.3 .2

Geopower

Geothermal Energy

Residual: $\quad 2.7$

Public health residuals

Recipient: $\quad 3.3 .4$

Social-Cultural domain

Quality of life

Potential Environmental Impact: A. Exploration

SE VERE

B. Construction

SEVERE

C. Exploitation

SEVERE

D. Transformation

MODERA TE

E. Transport

MODERATE

F. Waste disposal/processing

SEVERE

G. Termination

MODERATE

$\mathrm{H}$. Induced technology

UNASSESSED

Example Impacts: 1. A, B, C, D, E, F. Water quality (asbestos content) may expose public to carcinogens

Data Available: 1. EMDB

2. IVEP

3. BEIC

Models Availablę: 1. GRAPES

2. UMWQI

3. IUWQI

4. $\mathrm{NIH} / \mathrm{DF}-73-002$ 
Technology: $\quad 1.3 .2$

Geopower

Geothermal Energy

Residual: $\quad 2.7$

Public health residuals

Recipient: $\quad 3.3 .4 .1$

Social-Cultural domain

Quality of life

Health and safety

Potential Environmental Impact: A. Exploration

SEVERE

B. Construction

SEVERE

C. Exploitation

SEVERE

D. Transformation

MODERA TE

E. Transport

MINOR

F. Waste disposal/processing

SEVERE

G. Termination

MODERATE

H. Induced technology

UNASSESSED

Example Impacts: 1. A, B, C, D, E, F. Water quality (fluoride content) affects caries. in children

Data Available: 1. IVEP

2. BEIC

Models Available: 1. GRAPES

2. UMWQI

3. IUWQI

4. NIH/DF - $73-002$ 
Technology: $\quad 1.3 .2$

Geopower

Geothermal Energy

Residual: $\quad 2.7$

Public health residuals

Recipient: 3.3.4.2

Social-Cultural domain

Quality of life

Life style

Potential Environmental Impact:

A. Exploration

SEVERE

B. Construction

SE VERE

C. Exploitation

SEVERE

D. Transformation

MODERATE

E. Transport

MINOR

F. Waste disposal/processing

SEVERE

G. Termination

MODERATE

$\mathrm{H}$. Induced technology

UNASSESSED

Example Impacts: 1. A, B, C, D, E, F. Air quality (hydrogen sulfide, vehicle emissions) may impair residential values

Data Available:

Models Available: GRAPES 
Technology: $\quad 1.3 .2$

Geopower

Geothermal Energy

Residual: 2.7

Public health residuals

Recipient: $\quad 3.3 .4 .3$

Social-Cultural domain

Quality of life

Population characteristics

Potential Environmental Impac's: A. Exploration

SEVERE

B. Construction

SE VERE

C. Exploitation

SEVERE

D. Transformation

MODERATE

E. Transport

MODERATE

F. Waste disposal/processing

SE.VERE

G. Termination

MODERATE

H. Induced technology

UNASSESSED

Example Impacts: 1. A, B, C, D, E, F. Air quality may have unwonted effects on elderly

Data Available:

Models Available: 
Technology: $\quad 1.3 .2$

Geopower

Geothermal Energy

Residual: $\quad 2.7$

Public health residuals

Recipient: $\quad 3.3 .5$

Social-Cultural domain

Legal aspects

Potential Environmental Impact: A. Exploration

SE VERE

B. Construction

SEVERE

C. Exploitation

SEVERE

D. Transformation

MODERATE

E. Transport

SEVERE

F. Waste disposal/processing

SEVERE

G. Termination

MODERATE

H. Induced technology

UNASSESSED

Example Impacts: 1. A, B, C, D, E, F. Air and water quality may be in viola$\therefore . .$.

Data Available:

AEROS

Models Available: 
Technology: $\quad 1.3 .2$

Geopower

Geothermal Energy

Residual: $\quad 2.7$

Public health residuals

Recipient: 3.4

Economic domain

Potential Environmental Impact: A. Exploration

MODERA TE

B. Construction

SEVERE

C. Exploitation

SEVERE

D. Transformation

MODERATE

E. Transport

MINOR

F. Waste disposal/processing

SEVERE

G. Termination

MODERA TE

$\mathrm{H}$. Induced technology

UNASSESSED

Example Impacts: $\quad$ 1. A, B, C, D, E, F. Water quality may require improvement at public expense.

Data Available:

Models Available: 
Technology: $\quad 1.3 .2$

Geopower

Geothermal Energy

Residual: 2.7

Public health residuals

Recipient: 3.4 .1

Economic domain

Demographic factors

Potential Environmental Impact: A. Exploration

MODERATE

B. Construction

SEVERE

C. Exploitation

SEVERE

D. Transformation

MODERA TE

E. Transport

MINOR

F. Waste disposal/processing

SEVERE

G. Termination

MODERATE

$\mathrm{H}$. Induced technology

UNASSESSED

Example Impacts: 1. A, B, C, D, E, F. Water quality may require improvement at public expense.

Data Available:

4

Models Available:

B -173 
Technology: $\quad 1.3 .2$

Geopower

Geothermal Energy

Residual: 2.7

Public health residuals

Recipient: $\quad 3.4 .2$

Economic domain

Infrastructure domain

Potential Environmental Impact: A. Exploration

MODERATE

B. Construction

MODERATE

C. Exploitation

MODERATE

D. Transformation :

MODERATE

E. Transport

MINOR

F. Waste disposal/processing

SEVERE

G. Termination

MINOR

H. Induced technology

UNASSESSED

Example Impacts: 1. F. Water quality may place demands on local waste disposal.

Data Available:

Models Available: 
$m$
$\dot{\infty}$
$\stackrel{n}{*}$
$\dot{\sim}$
$\stackrel{\sim}{\sim}$

1 
Technology: $\quad 1.3 .2$

Geopower

Geothermal Energy

Residual: $\quad 2.8$

Infrastructure residuals

Recipient: $\quad 3.1$

Physical and chemical domain

Potential Environmental Impact: A. Exploration

MINOR

B. Construction

SEVERE

C. Exploitation

SEVERE

D. Transformation

MODERATE

E. Transport

MODERATE

F. Waste disposal/processing

MODERATE

G. Termination

MODERATE

H. Induced technology

UNASSESSED

Example Impacts: 1. A, B, C, D, E, F. Road and powerline construction denudes slopes and promotes erosion.

Data Available:

Models Available: 1. OSSIM

2. PSR V

3. CLEL 
Technology: $\quad 1.3 .2$

Geopower

Geothermal Energy

Residual: $\quad 2.8$

Infrastructure residuals

Recipient: 3.1 .1

Physical and chemical domain

Earth

Potential Environmental Impact: A. Exploration

MINOR

B. Construction

SEVERE

C. Exploitation

SEVERE

D. Transformation

MODERATE

E. Transport

MODERATE

F. Waste disposal/processing

MODERATE

G. Termination

MODERATE

H. Induced technology

UNASSESSED

Example Impacts: 1. A, B, C, D, E, F. Road construction affects topography.

Data Available:

Models Available: 1. PSRV

2. CLEL 
Technology: $\quad 1.3 .2$

Geopower

Geothermal Energy

Residual: 2.8

Infrastructure residuals

Recipient: 3.1 .1 .1

Physical and chemical domain

Earth

Topography

Potential Environmental Impact: A. Exploration

MINOR

B. Construction

SEVERE

C. Exploitation

SEVERE

D. Transformation

MODERATE

E. Transport

MODERATE

F. Waste disposal/processing

MODERATE

G. Termination

MODERATE

H. Induced technology

UNASSESSED

Example Impacts: 1. A, B, C, D,E, F. Road construction, power line pads, access roads affect topography.

2. G. Contours restored on termination.

Data Available:

Models Available: " 
Technology: $\quad 1.3 .2$

Geopower

Geothermal Energy

Residual: 2.8

Infrastructure residuals

Recipient: $\quad 3.1 .1 .2$

Physical and chemical domain

Earth

Resources

Potential Environmental Impact: A. Exploration

B. Construction

MINOR

C. Exploitation

MODERATE

D. Transformation

MODERATE

E. Transport

MINOR

F. Waste disposal/processing

MODERATE

G. Termination

MINOR

H. Induced technology

MINOR

UNASSESSED

Example Impacts: 1. A, B, C, D, E, F. Road building materials, fuel, impose drain on resources (aggregate, cement).

2. G. Recovery of pipeline steel relieves drain on resources.

Data Available:

Models Available: 
Technology: 1.3 .2

Geopower

Geothermal Energy

Residual: 2.8

Infrastructure residuals

Recipient: $\quad 3.1 .1 .3$

Physical and chemical domain

Earth

Slow processes

Potential Environmental Impact: A. Exploration

MINOR

B. Construction

MINOR

C. Exploitation

MINOR

D. Transformation

NONE

E. Transport

MINOR

F. Waste disposal/processing

NONE

G. Termination

MINOR

H. Induced technology

MINOR

Example Impacts: 1. A, B, C,E. Road, power line construction exposes bedrock, accelerating weathering.

Data Available:

Models Available: 
Technology: $\quad 1.3 .2$

Geopower

Geothermal Energy

Residual: $\quad 2.8$

Infrastructure residuals

Recipient: 3.1 .1 .3 .1

Physical and chemical domain

Earth

Slow processes

Weathering

Potential Environmental Impact: A. Exploration

MINOR

B. Construction

MINOR

C. Exploitation

MINOR

D. Transformation

NONE

E. Transport

MINOR

F. Waste disposal/processing NONE

r. Termination

MINOR

H. Induced technology

MINOR

Example Impacts: 1. A, B, C,E. Road, power line construction exposes bedrock, accelerating weathering.

Data Available:

Models Available: 
Technology: $\quad 1.3 .2$

Geopower

Geothermal Energy

Residual: $\quad 2.8$

Infrastructure residuals

Recipient: 3.1 .1 .4

Physical and chemical domain

Earth

Rapid processes

Potential Environmental Impact: A. Exploration

MINOR

B. Construction

MINOR

C. Exploitation

MINOR

D. Transformation

NONE

E. Transport

MINOR

F. Waste disposal/processing

MINOR

G. Termination

MINOR

H. Induced technology

UNASSESSED

Example Impacts: 1. A, B, C,E, F. Road and powerline construction denudes slopes and promotes landslides.

Data Available:

Models Available: 1. PSRV

2. CLEL 
Technology: $\quad 1.3 .2$

Geopower

Geothermal Energy

Residual: $\quad 2.8$

Infrastructure residuals

Recipient: 3.1 .1 .4 .1

Physical and chemical domain

Earth

Rapid processes

Flood

Potential Environmental Impact: A. Exploration

MINOR

B. Construction

MINOR

C. Exploitation

MINOR

D. Transformation

NONE

E. Transport

MINOR

F. Waste disposal/processing NONE

G.'Termination

MINOR

H. Induced technology

UNASSESSED

Example Impacts: 1. A, B, C, D, E. Road and powerline construction denudes slopes and promotes flooding:

Data Available:

Models Available: 
Technology: $\quad 1.3 .2$

Geopower

Geothermal Energy

Residual: - 2.8

Infrastructure residuals

Recipient: 3.1 .1 .4 .2

Physical and chemical domain

Earth

$$
\begin{gathered}
\text { Rapid processes } \\
\text { Erosion }
\end{gathered}
$$

Potential Environmental Impact: A. Exploration

MINOR

B. Construction

MINOR

C. Exploitation

MINOR

D. Transformation

NONE

E. Transport

MINOR

F. Waste disposal/processing

MINOR

G. Termination

MINOR

H. Induced technology

UNASSESSED
Example Impacts: 1. A, B, C, E, F. Road and powerline construction denudes slopes and promotes erosion.

Data Available:

Models Available: 
Technology: 1.3 .2

Geopower

Geothermal Energy

Residual: 2.8

Infrastructure residuals

Recipient: 3.1.1.4.4

Physical and chemical domain

Earth

Rapid processes

Land slide/Avalanche

Potential Environmental Impact: A. Exploration

MINOR

B. Construction

MINOR

C. Exploitation

MINOR

D. Transformation

NONE

E. Transport

MINOR

F. Waste disposal/processing

NONE

r. Termination

MINOR

H. Induced technology

UNASSESSE D

Example Impacts: 1. A, B, C, E. Road and powerline construction may induce landslides.

Data Available:

Models Available: 
Technology: 1.3 .2

Geopower

\section{Geothermal Energy}

Residual: 2.8

Infrastructure residuals

Recipient: 3.1 .1 .4 .6

Physical and chemical domain

Earth

Rapid processes

Air movement

Potential Environmental Impact: A. Exploration

B. Construction

NONE

C. Exploitation

MINOR

D. Transformation

MINOR

E. Transport

MINOR

F. Waste disposal/processing

NONE

G. Termination

MINOR

$\mathrm{H}$. Induced technology

MINOR

NONE

Example Impacts: 1. B, C, D, E. Tall structures transverse to wind flow impede circulation.

Data Available:

Models Available: 
Technology: $1: 3.2$

Geopower

Geothermal Energy

Residual: 2.8

Infrastructure residuals

Recipient: 3.1 .2

Physical and chemical domain

Water

Potential Environmental Impact: A. Exploration

MINOR

B. Construction

MINOR

C. Exploitation

MODERATE

D. Transformation

MINOR

E. Transport

MINOR

F. Waste disposal/processing

MINOR

G. Termination

MINOR

H. Induced technology

UNASSESSED

Example Impacts: A, B, C, D, E, F. Provision of a public service (domestic water) draws upon local water supplies.

Data Available:

Models Available: 
Technology: 1.3 .2

Geopower

Geothermal Energy

Residual: 2.8

Infrastructure residuals

Recipient: 3.1 .3

Physical and chemical domain

Air

Potential Environmental Impact: A. Exploration

MINOR

B. Construction

MODERATE

C. Exploitation

MODERATE

D. Transformation

MINOR

E. Transport

MODERATE

F. Waste disposal/processing

MINOR

G. Termination

MINOR

$\mathrm{H}$. Induced technology

UNASSESSED

Example Impacts: 1. A, B, C, D, E, E. Emissions from transportation systems degrade air quality.

Data Available:

Models Available: OSSIM 
Technology: 1.3 .2

Geopower

Geothermal Energy

Residual: 2.8

Infrastructure residuals

Recipient: $\quad 3.2$

Biological domain

Potential Environmental Impact: A. Exploration

MODERA TE

B. Construction

MODERATE

C. Exploitation

MODERA TE

D. Transformation

MODERATE

E. Transport

SE VERE

F. Waste disposal/processing

G. Termination

MODERATE

$\mathrm{H}$. Induced technology

MINOR

UNASSESSED

Example Impacts: 1. E. Road system exposes animals to death by vehicles.

Data Available:

Models Available: 
Technology: $\quad 1.3 .2$

\section{Geopower}

Geothermal Energy

Residual: 2.8

Infrastructure residuals

Recipient: 3.2 .1 .

Biological domain

Animals

Potential Environmental Impact: A. Exploration

MODERATE

B. Construction

MODERATE

C. Exploitation

MODERATE

D. Transformation

MODERATE

E. Transport

SEVERE

F. Waste disposal/processing

MODERA TE

G. Termination

MINOR

H. Induced technology

UNASSESSED

Example.Impacts: 1. A, B,C, D,E, F. Road system disrupts migration paths. Exposes animals to death by vehicles.

2. E. Birds killed by power lines.

Data Available:

Models Available: 
Technology: $\quad 1.3 .2$

Geopower

Geothermal Energy

Residual: 2.8

Infrastructure residuals

Recipient: 3.2 .2

Biological domain

Plants

Potential Environmental Impact: A. Exploration

MINOR

B. Construction

MINOR

C. Exploitation

MINOR

D. Transformation

MINOR

E. Transport

MODERATE

F. Waste disposal/processing MINOR

G. Termination

MINOR

H. Induced technology

UNASSESSE D

Example Impacts: 1. A, B, C, D, E, F. Road system requires clearing of vegetation.

Data Available:

Models Available: 
Technology: $\quad 1.3 .2$

Geopower

Geothermal Energy

Residual: 2.8

Infrastructure residuals

Recipient: 3.3

Social-cultural dómain

Potential Environmental Impact: A. Exploration

MODERATE

B. Construction

SEVERE

C. Exploitation

SEVERE

D. Transformation

SEVERE

E. Transport

MODERATE

F. Waste disposal/processing

SEVERE

G. Termination

MODERATE

$H$. Induced technology

UNASSESSED

Example Impacts: 1. A, B, C, D, E, F. Diversion of previous land use for structures, roads, power lines.

Data Available: $\quad$ 1. EMDB

2. SITE

3. GDB

4. $\mathrm{COM} / \mathrm{DF}-73-022$

5. $\mathrm{DOI} / \mathrm{DF}-73-001$

Models Available: 1. SRIEM

2. OSSIM

3. CES/HNL2 
Technology: $\quad 1.3 .2$

Geopower

Geothermal Energy

Residual: $\quad 2.8$

Infrastructure residuals

Recipient: 3.3 .1

Social-cultural domain

Land use

Potential Environmental Impact: A. Exploration

MINOR

B. Construction

SEVERE

C. Explötātion

SE VERE

D. Transformation

SEVERE

E. Transport

MINOR

F. Waste disposal/processing

MODERA TE

G. Termination

MODERATE

$\mathrm{H}$. Induced technology

UNASSESSE D

Example Impacts: 1. A, B, C, D, E, F. Diversion of previous land use for structures, roads, power lines.

Data Available: 1. EMDB

2. SITE

3. GDB

4. $\mathrm{COM} / \mathrm{DF}-73-022$

Models Available: 1. OSSIM

2. SKIEM 
Technology: 1.3 .2

Geopower

Geothermal Energy

Residual: 2.8

Infrastructure residuals

Recipient: 3.3 .2

Social-cultural domain

Recreation

Potential Environmental Impact: A. Exploration

MINOR

B. Construction

SEVERE

C. Exploitation

SEVERE

D. Transformation

MODERATE

E. Transport

MODERATE

F. Waste disposal/processing

SE VERE

G. Termination

MODERA TE

H. Induced technology

UNASSESSED

Example Impacts: 1. A, B, C, D, E, F. Loss of hunting, fishing, by

construction of structures, roads.

Data Available:

DOI/DF - 73-001

Models Available: 
Technology: $\quad 1.3 .2$

Geopower

Geothermal Energy

Residual: 2.8

Inf rastructure residuals

Recipient: 3.3 .3

Social-cultural domain

Aesthetics

Potential Environmental Impact: A. Exploration

MINOR

B. Construction

SEVERE

C. Exploitation

SEVERE

D. Transformation

SEVERE

E. Transport

MODERATE

F. Waste disposal/processing

SEVERE

G. Termination

MODERATE

H. Induced technology

UNASSESSED Example Impacts: 1. A. Temporary housing, storage structures degrade
aesthetic values of wilderness areas.

Data Available:

Modèls Available: 
Technology: 1.3 .2

Geopower

Geothermal Energy

Residual: 2.8

Infrastructure residuals

Recipient: $\quad 3.3 .4$

Social-cultural domain

Quality of life

Potential Environmental Impact: A. Exploration

MINOR

B. Construction

MODERATE

C. Exploitation

MODERATE

D. Transformation

MINOR

E. Transport

MODERATE

F. Waste disposal/processing

MODERATE

G. Termination

MODERATE

H. Induced technology

UNASSESSED

Example Impacts: 1. A, B, $\grave{C}, D, E, F$. Improvements in public services may benefit life style of rural popultations.

Data Available:

Models Available: 
Technology: $\quad 1.3 .2$

Geopower

Geothermal Energy

Residual: 2.8

Infrastructure residuals

Recipient: 3.3 .4 .1

Social-cultural domain

Quality of life

Health and safety

Potential Environmental Impact: A. Exploration

NONE

B. Construction

MINOR

C. Exploitation

MINOR

D. Transformation

NONE

E. Transport

MINOR

F. Waste disposal/processing

MINOR

G. Termination

MINOR

H. Induced technology

UNASSESSED D

Example Impacts: 1. B, C,E, F. Road system results in atmospheric emissions, causing health problems.

2. B, C, E, F. Road system may result in vehicular deaths.

Data Available:

Models Available: 
Technology: $\quad 1.3 .2$

Geopower

Geothermal Energy

Residual: 2.8

Infrastructure residuals

Recipient: 3.3 .4 .2

Social-cultural domain

Quality of life

Life s.tyle

Potential Environmental Impact: A. Exploration

MINOR

B. Construction

MODERATE

C. Exploitation

MODERATE

D. Transformation

MINOR

E. Transport

MODERA TE

F. Waste disposal/processing

MODERATE

G. Termination

MODERATE

$\mathrm{H}$. Induced technology

UNASSESSED

Example Impacts: 1. A, B, C, D, E, F. Improvement in public services may benefit life style o:f rural populations.

Data Available:

Models Available: 
Technology: $\quad 1.3 .2$

Geopower

Geothermal Energy

Residual: 2.8

Infrastructure residuals

Recipient: $\quad 3.3 .5$

Social-cultural domain

Legal aspects

Potential Environmental Impact: A. Exploration

MODERATE

B. Construction

MODERATE

C. Exploitation

MODERATE

D. Transformation

MINOR

E. Transport

MODERATE

F. Waste disposal/processing

MODERATE

G. Termination

MODERATE

H. Induced technology

UNASSESSED

Example Impacts: 1. A, B, C, D,E, F. Permits, licenses, insurance required for roads, communication systems, public services.

Data Available:

Models Available: 1. SRIEM

2. CES/HNL2 
Technology: $\quad 1.3 .2$

Geopower

Geothermal Energy

Residual: $\quad 2.8$

Infrastructure residuals

Recipient: 3.4

Economic domain

Potential Environmental Impact: A. Exploration

MODERATE

B. Construction

SEVERE

C. Exploitation

SEVERE

D. Transformation

MODERATE

E. Transport

MINOR

F. Waste disposal/processing

SEVERE

G. Termination

MODERATE

H. Induced technology

UNASSESSED

Example Impacts: 1. A, B, C, D, E, F. Communications requirements may overload rural telephone systems.

Data Available: 1. OBERS

2. IVEP

3. $\mathrm{EMDB}$

4. TRIS

Models Available: 1. OSSIM

7. IEES

2. PIES

8. TRIS

3. SRIEM

9. CES/HNI ?

4. ESPM

10. CES/HNL 1

5. WISE

6. MIROH 
Technology: $\quad 1.3 .2$

Geopower

Geothermal Energy

Residual: 2.8

Infrastructure residuals

Recipient: 3.4 .1

Economic domain

Demographic factors

Potential Environmental Impact: A. Exploration

MINOR

B. Construction

MINOR

C. Exploitation

MINOR

D. Transformation

MINOR

E. Transport

MINOR

F. Waste disposal/processing

MINOR

G. Termination

MINOR

H. Induced technology

UNASSESSED

Example Impacts: 1. A, B, C, D, E, F. Demand for public services requires public expenditures.

Data Available:

OBERS

Models Available: 1. OSSIM

2. PIES

3. SRIEM

4. WISE

5. CES/HNLl

6. CES/HNL2 
Technology: $\quad 1.3 .2$

Geopower

Geothermal Energy

Residual: $\quad 2.8$

Infrastructure residuals

Recipient: 3.4 .2

Economic domain

Infrastructure domain

Potential Environmental Impact: A. Exploration

MODERA TE

B. Construction

SEVERE

C. Exploitation

SEVERE

D. Transformation

MODERATE

E. Transport

MINOR

F. Waste disposal/processing

SEVERE

G. Termination

MODERATE

H. Induced technology

UNASSESSED

Example Impacts: 1. A, B, C, D, E, F. Transporation requirements for heavy equipment may damage road system.

Data Available: . 1. OBERS

2. EMDB

3. IVEP

4. TRIS

Models Available: 1. OSSIM

7. IEES

2. PIES

8. TRIS

3. SRIEM

4. F.SPM

5. MIROH

6. WISE 
$\boldsymbol{D}$

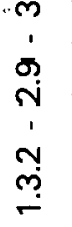

P 
Technology: 1.3 .2

Geopower

Geothermal Energy

Residual: 2.9

Catastrophic residuals

Recipient: 3.1

Physical and chemical domain

Potential Environmental Impact: A. Exploration

SE VERE

B. Construction

SEVERE

C. Exploitation

SEVERE

D. Transformation

SEVERE

E. Transport

F. Waste disposal/processing

SEVERE

G. Termination

SEVERE

$H$. Induced technology

MINOR

UNASSESSED

Example Impacts: $\quad$ 1. B, C, D,E, F. Earthquake triggered by reinjection may change topography with resulting impacts on all structures.

Data Available:

1. $\mathrm{COM} / \mathrm{DF}-73-008$

2. USGS/HAMILTON

3. ERDA-2893

4. ERDA -2895

5. ERDA -2900

6. ERDA -2907

7. UCR/COMBS

8. TAMU/WHITING

9. A.FROS

Models Available: 1. PSRV

2. CLEL 
Technology: $\quad 1.3 .2$

Geopower

Geothermal Energy

Residual: $\quad 2.9$

Catastrophic residuals

Recipient: 3.1 .1

Physical and chemical domain

Earth

Potential Environmental Impact: A. Exploration

SEVERE

B. Construction

SEVERE

C. Exploitation

SEVERE

D. Transformation

SEVERE

E. Transport

SEVERE

F. Wastè disposal/processing

SEVERE

G. Termination

MINOR

$\mathrm{H}$. Induced technology

UNASSESSED

Example Impacts: 1. B, C, D, E, F. Earthquake triggered by reinjection may change topography with resulting impacts on all structures.

Data Available: 1. COM/DF-73-008

2. USGS/HAMILTON

3. ERDA -2893

4. ERDA -2895

5. ERDA -2900

6. ERDA-2907

7: UCR/COMBS

8. TAMU/WHITING

Models Available: 1. PSRV

2. CLEL 
Technology: $\quad 1.3 .2$

Geopower

Geothermal Energy

Residual: $\quad 2.9$

Catastrophic residuals

Recipient: 3.1 .1 .1

Physical and chemical domain

Earth

Topography

Potential Environmental Impact: A. Exploration

MINOR

B. Construction

SEVERE

C. Exploitation

SEVERE

D. Transformation

SEVERE

E. Transport

SE VERE

F. Waste disposal/processing

SEVERE

G. Termination

NONE

H. Induced technology

UNASSESSED

Example Impacts: 1. B, C, D, E, F. Earthquake may change topography with resulting impacts on all structures.

Data Available:

Models Available: 
Technology: $\quad 1.3 .2$

Geopower

Geothermal Energy

Residual: 2.9

Catastrophic residuals

Recipient: 3.1 .1 .2

Physical and chemical domain

Earth

Resuurces

Potential Environmental Impact: A. Exploration

MODERA TE

B. Construction

NONE

C. Exploitation

MODERA TE

D. Transformation

NONE

E. Transport

NONE

F. Waste disposal/processing

NONE

G. Termination

NONE

$\vec{H}$. Induced technology

INOINE

Example Impacts: 1. A, C. Blowout contributes to depletion of geothermal resource.

Data Available:

Models Available: 
Technology: 1.3 .2

Geopower

Geothermal Energy

Residual: 2.9

Catastrophic residuals

Recipient: 3.1 .1 .3

Physical and chemical domain

Earth

Slow processes

Potential Environmental Impact: A. Exploration

MINOR

B. Construction

NONE

C. Exploitation

MINOR

D. Transformation

NONE

E. Transport

NONE

F. Waste disposal/processing

NONE

G. Termination

NONE

$\mathrm{H}$. Induced technology

NONE

Example Impacts: 1. A,C. Blowout may contribute to subsidence.

2. C, F. Earthquake triggered by reinjection promotes weathering by fracturing rock.

Data Available:

Models Available: 
Technology: $\quad 1.3 .2$

Geopower

Geothermal Energy

Residual: $\quad 2.9$

Catastrophic residuals

Recipient: .3.1.1.3.1

Physical and chemical domain

Earth

Slow processes

Weathering

Potential Environmental Impact: A. Exploration . NONE

B. Conetruction NONE

C. Exploitation MINOR

D. Transformation NONE

E. Transport NONE

F. Waste disposal/processing NONE

G. 'T'ermination NONE

H. Induced technology NONE

Example Impacts: 1. C, F. Earthquake triggered by reinjection promotes weathering by fracturing rock.

Data Available:

Models Available: 
Technulogy: $\quad 1.3 .2$

Geopower

Geothermal Energy

Residual: 2.9

Catastrophic residuals

Recipient: 3.1 .1 .3 .6

Physical aud ehemical domain

Earth

Slow processes

Subsidence

Potential Environmental Impact: A. Exploration

MINOR

B. Construction

NONE

C. Exploitation

MINOR

D. Transformation

NONE

E. Transport

NONE

F. Waste disposal/processing

NONE

G. Termination

NONE

$\mathrm{H}$. Induced technology

NONE

Example Impacts: 1. A, C. Blowout may contribute to subsidence.

Data Available:

Models Available: 
Technology: $\quad 1.3 .2$

Geopower

Geothermal Energy

Residual: 2.9

Catastrophic residuals

Recipient: 3.1 .1 .4

Physical and chemical domain

Earth

Rapid processes

Potential Environmental Impact: A. Exploration

SEVERE

B. Construction

SEVERE

C. Exploitation

SEVERE

D. Transformation

NONE

E. Transport

SEVERE

F. Waste disposal/processing

SEVERE

G. Termination

MINOR

H. Induced technology

UNASSESSED

Example Impacts: 1. C,F. Reinjection of geothermal fluids may unlock faults and trigger seismic movement.

Data Available: $\quad$ 1. COM/DF-73-008.

2. USGS/HAMILTON

3. ERDA -2893

4. ERDA -2895

5. ERDA -2900

6. ERDA -2907

7. UCR/COMBS

8. TAMU /WHITING

Models Available: 1. PSRV

2. CLEL 
Technology: $\quad 1.3 .2$

Geopower

Geothermal Energy

Residual: 2.9

Catastrophic residuals

Recipient: 3.1 .1 .4 .1

Physical and chemical domain

Earth

Rapid processes

Flood

Potential Environmental Impact: A. Exploration

SEVERE

B. Construction

SEVERE

C. Exploitation

SEVERE

D. Transformation

NONE

E. Transport

SEVERE

F. Waste disposal/processing

NONE

G. Termination

MINOR

H. Induced technology

UNASSESSED

Example Impacts: 1. A, B, C, E, G. Fire denudes slopes and may result in flood.

Data Available:

Models Available: 
Technology: 1.3 .2

Geopower

Geothermal Energy

Residual: $\quad 2.9$

Catastrophic residuals

Recipient: $\quad 3.1 .1 .4 .2$

Physical and chemical domain

Earth

$$
\begin{gathered}
\text { Rapid processes } \\
\text { Erosion }
\end{gathered}
$$

Potential Environmental Impact: A. Exploration

SEVERE

B. Construction

SE VERE

C. Exploitation

SEVERE

D. Transformation

NONE

E. Transport

SE VERE

F. Waste disposal/processing

NONE

G. Termination

MINOR

H. Induced technology

UNASSESSED

Example Impacts: 1. A, B, C, E, G. Fire denudes slopes and may result in erosion.

Data Available:

Models Available: 
Technology: $\quad 1.3 .2$

Geopower

Geothermal Energy

Residual: ' 2.9

Catastrophic residuals

Recipient: $\quad 3.1 .1 .4 .4$

Physical and chemical domain

Earth

$$
\begin{aligned}
& \text { Rapid processes } \\
& \text { Landslide/Avalanche }
\end{aligned}
$$

Potential Environmental Impact: A. Exploration

NONE

B. Construction

NONE

C. Exploitation

SEVERE

D. Transformation

NONE

E. Transport

NONE

F. Waste disposal/processing

SEVERE

G. Termination

NONE

H. Induced technology

NONE

Example Impacts: 1. C,F. Earthquake causes landslides and avalanches.

Data Available:

Models Available:

B -213 
Technology: 1.3 .2

Geopower

Geothermal Energy

Residual: $\quad 2.9$

Catastrophic residuals

Recipient: 3.1 .1 .4 .5

Physical and chemical domain

Earth

Rapid processes

Seismicity

Potential Environmental Impact: A. Exploration NONE

B. Construction NONE

C. Exploitation SEVERE

D. Transformation NONE

E. Transport NONE

F. Waste disposal/processing SEVERE

G. Termination NONE

II. Indueed technology NONE

Example Impacts: 1. C,F. Reinjection of geothermal fluids may unlock faults and trigger seismic movement.

Data Available: 1. COM/DF-73-008

2. USGS/HAMILTON

3. ERDA -2893

4. ERDA -2895

5. ERDA-2900

6. ERDA -2907

7. UCR/COMBS

8. TAMU/WHITING

Models Available: 1. PSRV

2. CLEL 
Technology: $\quad 1.3 .2$

Geopower

Geothermal Energy

Residual: $\quad 2.9$

Catastrophic residuals .

Recipient: 3.1 .1 .4 .6

Physical and chemical domain

Earth

Rapid processes

Air movement

Potential Environmental Impact: A. Exploration

NONE

B. Construction

MINOR

C. Exploitation

NONE

D. Transformation

NONE

E. Transport

NONE

$\dot{F}$. Waste disposal/processing

NONE

G. Termination

NONE

$\mathrm{H}$. Induced technology

NONE Example Impacts: 1. B. Shock wave from "blasting may injure personnel,

Data Available:

Models Available: 
Technology: $\quad 1.3 .2$

Geopower

Geothermal Energy

Residual: $\quad 2.9$

Catastrophic residuals

Recipient: 3.1 .1 .5

Physical and chemical domain

Earth

Unique features

Potential Environmental Impact: A. Exploration

MINOR

B. Construction

NONE

C. Exploitation

MINOR

D. Transformation

NONE

E. Transport

NONE

F. Waste disposal/processing

MINOR

G. Termination

NONE

$\mathrm{H}$. Induced technology

NONE

Example Impacts: 1. A, C, F. Blowout lowers aquifer pressure.

Data Available:

Models Available: 
Technology: $\quad 1.3 .2$

Geopower

Geothermal Energy

Residual: $\quad 2.9$

Catastrophic residuals

Recipient: $\quad 3.1 .2$

Physical and chemical domain

Water

Potential Environmental Impact: A. Exploration

MODERATE

B. Construction

NONE

C. Exploitation

MODERATE

D. Transformation

NONE

E. Transport

NONE

F...Waste disposal/processing

MINOR

G. Termination

NONE

H. Induced technology

NONE

Example Impacts: 1. A, C, F. Blowout may pollute surface and ground water.

Data Available:

Models Available: 
Technology: 1.3 .2

Geopower

Geothermal Energy

Residual: $\quad 2.9$

Catastrophic residuals

Recipient: $\quad 3.1 .3$

Physical and chemical domain

Air

Potential Environmental Impact: A. Exploration

MODERA TE

B. Construction

NONE

C. Exploitation

MODERATE

D. Transformation

NONE

E. Transport

NONE

F. Waste disposal/processing

MINOR

G. Termination

NONE

H. Induced te elinology

NONE

Example Impacts: 1. A,C.F. Blowout may pollute air.

Data Available:

AEROS

Models Available: 
Technology: 1.3 .2

Geopower

Geothermal Energy

Residual: $\quad 2.9$

Catastrophic residuals

Recipient: $\quad 3.2$

Biological domain

Potential Environmental:Impact: A. Exploration

MODERATE

B. Construction

NONE

C. Exploitation

MODERATE

D. Transformation

NONE

E. Transport

NONE

F. Waste disposal/processing

MINOR

G. Termination

NONE

H. Induced technology

NONE

Example Impacts: 1. A, C, F. Blowout kills plant life, drives away animals.

Data Available:

Models Available: 
Technology: $\quad 1.3 .2$ Geopower

Geothermal Energy

Residual: 2.9

Catastrophic residuals

Recipient: $\quad 3.2 .1$

Biological domain

Animals

Potential Environmental Impact: A. Exploration

MODERATE

B. Construction

NONE

C. Exploitation

MODERATE

D. Transformation

NONE

E. Transport

NONE

F. Waste disposal/processing

MINOR

G. Termination

NONE

H. Indueed technology

NONE

Example Impacts: 1. A, C, F. Blowout drives away animals.

Data Available:

Models Available: 
Technology: 1.3 .2 Geopower

Geothermal Energy

Residual: 2.9

Catastrophic residuals

Recipient: $\quad 3.2 .2$

Biological domain

Plants

Potential Environmental Impact: A. Exploration

MODERATE

B. Construction

NONE

C. Exploitation

MODERATE

D. Transformation

NONE

E. Transport

NONE

F. Waste disposal/processing

MINOR

G. Termination

NONE

H. Induced technology

NONE

Example Impacts: 1. A, C, F. Blowout kills plant life.

Data Available:

Models Available: 
Technology: 1.3 .2

Geopower

Geothermal Energy

Residual: $\quad 2.9$

Catastrophic residuals

Recipient: . 3.3

Social-cultural domain

Potential Environmental Impact: A. Exploration

SEVERE

B. Construction

SEVERE

r., Fixplnitation

SEVERE

D. Transformation

NONE

E. Transport

NONE

F. Waste disposal/processing

MINOR

G. Termination

NONE

H. Induced lechnology

NONE

Example Impacts: 1. A, B, C, F. All catastrophic residuals may evoke constraining and punitive legal action.

Data Available:

Models Available: 
Technology: $\quad 1.3 .2$

Geopower

Geothermal Energy.

Residual: 2.9

Catastrophic residuals

Recipient: $\quad 3.3 .1$

Social-cultural domain

Land use

Potential Environmental Impact: A. Exploration

MODERA TE

B. Construction

MINOR:

C. Exploitation

MODERATE

D. Transformation

NONE

E. Transport

NONE

F. Waste disposal/processing

MINOR

G. Termination

NONE

$\mathrm{H}$. Induced technology

NONE

Example Impacts: 1. A, C, F. Induced seismicity may make area unsuited for certain land uses, e.g., residential, commercial, industrial.

Data Available:

Models Available: 
Technology: $\quad 1.3 .2$

Geopower

Geothermal Energy

Residual: 2.9

Catastrophic residuals

Recipient: $\quad 3.3 .2$

Social-cultural domain

Recreation

Potential Environmental Impact: A. Exploration

MODERATE

B. Construction

MINOR

C. Exploitation

MODERATE

D. Transformation

NONE

E. Transport

NONE

F. Waste disposal/processing

MINOR

G. Termination

NONE

$\mathrm{H}$. Induced téchnology

INONE

Example Impacts: 1. A, B, C, F. Induced seismicity may make area unsuited for recreation.

Data Available:

Models Available: 
Technology: 1.3 .2

Geopower

Geothermal Energy

'Residual: 2.9

Catastrophic residuals

Recipient: $\quad 3.3 .3$

Social-cultural domain

Aesthetics

Potential Environmental Impact: A. Exploration

MODERATE

B. Construction

NONE

C. Exploitation

MODERATE

D. Transformation

NONE

E. Transport

NONE

F. Waste disposal/processing

MINOR

G. Termination

NONE

H. Induced technology

NONE

Example Impacts: 1. A, C, F. Induced seismicity impairs aesthetic appreciation of wilderness a rea.

Data Available:

Models Available: 
Technology: $\quad 1.3 .2$

Geopower

\section{Geothermal Energy}

Residual: $\quad 2.9$

Catastrophic residuals

Recipient: $\quad 3.3 .4$

Social-cultural domain

Quality of life

Potential Environmental Impact: A. Exploration

SEVERE

B. Construction

NONE

C. Explnitation

SEVERE

D. Transformation

NONE

E. Transport

NONE

F. Waste disposal/processing

MODERATE

G. Termination

NONE

H. 'Induced technolugy

NONE

Example Impacts: 1. A, C, F. Induced seismicity a safety hazard.

2. A, C, F. Blowouts a safety hazard.

Data Available:

Models Available: 
Technology: 1.3 .2

Geopower

Geothermal Energy

Residual: 2.9

Catastrophic residuals

Recipient: 3.3 .4 .1 .

Social-cultural domain

Quality of life

Health and safety

Potential Environmental Impact: A. Exploration

SEVERE

B. Construction

NONE

C. Exploitation

SEVERE

D. Transformation

NONE

E. Transport

NONE

F. Waste disposal/processing

MODERATE

G. Termination

NONE

H. Induced technology

NONE

Example Impacts: 1. A, C,F. Induced seismicity a safety hazard.

Data Available:

Models Available: 
Technology: $\quad 1.3 .2$

Geopower

Geothermal Energy

Residual: 2.9

Catastrophic residuals

Recipient: 3.3 .4 .2

Social-cultural domain

Quality of life

Life style

Potential Environmental Impact:

A. Exploration

MODERATE

B. Construction

NONE

C. Expluilation

MODERATE

D. Transformation

NONE

E. Transport

NONE

F. Waste disposal/processing

MINOR

G. Termination

NONE

$\mathrm{H}$. Induced technology

NONE

Example Impacts: 1. A, C,F. Induced seismicity creates anxieties that may dis rupt life style.

Data Available:

Models Available: 
Technology: $\quad 1.3 .2$

Geopower

Geothermal Energy

Residual: $\quad 2.9$

Catastrophic residuals

Recipient: $\quad 3.3 .4 .3$

Social-cultural domain

Quality of life

Population characteristics

Potential Environmental Impact: A. Exploration

MODERA TE

B. Construction

NONE

C. Exploitation

MODERATE

D. Transformation

NONE

E. Transport

NONE

F. Waste disposal/processing

G. Termination

MINOR

H. Induced technology

NONE

NONE

Example Impacts: 1. A, C, F. Strong induced seismicity may cause population shifts.

Data Available:

Models Available: 
Technology: 1.3 .2

Geopower

Geothermal Energy

Residual: $\quad 2.9$

Catastrophic residuals

Recipient: $\quad 3.3 .5$

Social-cultural domain

Legal aspects

Potential Environmental Impact: A. Exploration

SEVERE

B. Construction

SEVERE

C. Exploitation

SEVERE

D. Transformation

NONE

E. Transport

NONE

F. Waste disposal/processing

SE VERE

G. Termination

NONE

H. Induced technology

NONE

Example Impacts: 1. A, B, C,F. All catastrophic residuals may evoke legal action.

Data Available:

Models Available: 
Technology: 1.3 .2

Geopower

Geothermal Energy

Residual: 2.9

Catastrophic residuals

Recipient: $\quad 3.4$

Economic domain

Potential Environmental Impact: A. Exploration

MODERATE

B. Construction

MODERA TE

C. Exploitation

MODERATE

D. Transformation

MODERA TE

E. Transport

MODERATE

F. Waste disposal/processing

MODERATE

G. Termination

NONE

H. Induced technology

NONE

Example Impacts: 1. A, B, C, D, E, F. All catastrophic residuals may affect structures, communications.

Data Available:

Models Available: 
Technology: $\quad 1.3 .2$

Geopower

Geothermal Energy

Residual: 2.9

Catastrophic residuals

Recipient: 3.4.1

Economic domain

Demographic factors

Potential Environmental Impact: A. Exploration

MODERATE

B. Construction

MODERATE

C. Exploitation

MODERATE

D. Transformation

MODERATE

E. Transport

MODERATE

F. Waste disposal/processing

MODERATE

G. Termination

NONE

H. Induced technology

NONE

Example Impacts: 1. A; B, C, D, E, F. All catastrophic residuals may affect wage rates.

Data Available:

Models Available: 
Technology: $\quad 1.3 .2$

Geopower

Geothermal Energy

Residual: $\quad 2.9$

Catastrophic residuals

Recipient: $\quad 3.4 .2$

Economic domain

Infrastructure domain

Potential Environmental Impact: A. Exploration

MODERA TE

B. Construction

MODERATE

C. Exploitation

MODERATE

D. Transformation

MODERA TE

E. Transport

MODERA TE

F. Waste disposal/processing

MODER A TE

G. Termination

NONE

H. Induced technology

NONE

Example Impacts: 1. A, B, C, D, E, F. All catastrophic residuals may affect structures, communications.

Data Available:

Models Available: 



\section{APPENDIX C}

\section{GEOTHERMAL ENVIRONMENTAL IMPACT DIRECTORY SYSTEM}

MODEL AND DATA BASE DIRECTOR Y 


\section{Model: ADPIC}

Title: Atmospheric Diffusion Particle-in-Cell, A 3-dimensional Diffusion Transport Model

Date: 1973

Geographic Area: not applicable

Geographic Coordinates: not applicable

FIPS Code: not applicable

Abstract: ADPIC is a 3-dimensional, cartesian diffusion-transport code based on the particle-in-cell and pseudo-velocity principles: It is capable of calculating the time-dependent distribution of inert or radioactive pollutants in the atmosphere under many conditions, including stratified shear flow, calms, topographical intrusion, wet and dry deposition, for instantaneous and continuous sources. The pollutant concentrations are represented by Lagrangian marker particles moving in an Eulerian grid. The model accepts any mass consistent advection field.

Assessment: None

Analyses Performed: ADPIC--A 3-Dimensional Transport-Diffusion Model, Rolf Lange, UCRL (preprint) 76170, 1975.

Funding: unknown

Model Availability: ADPIC--A 3-Dimensional Computer Code, Rolf Lange, UCRL-51462 (1973).

Archive Medium: hard copy

Contact: Rolf Lange or Leonard Laws on G-Division

Lawrence Livermore Laboratory

P. O. Box 808

Livermore, CA 94550

Master Control No. 30059

Citations: $1.3 .2--2.3--3.1 .1$

$$
\text { C. }-1
$$


Data Base: AEROS

Title: Aerometric and Emissions Reporting System

Date:

Geographic Area: national on a locality or point source basis

Geographic Coordinates: not applicable

FIPS Code: not applicable

Abstract: The Aerometric and Emissions Reporting System (AEROS) serves as a management information system for EPA's air pollution research and control programs. As such, AEROS is mainly concerned with the collection, processing, and reporting of basic air pollution data. Various supplementary data files are also maintained to provide additional informatinn valuable for the preparation and analysis of air pollution data. The principal subsystems of AEROS are:

1. The National Emissions Data System (NEDS) which stores and reports source and emissions-related data for the five criteria pollutants (particulates, $\mathrm{SO}_{\mathrm{x}}, \mathrm{NO}_{\mathrm{x}}, \mathrm{CO}$, and hydrocarbons).

2. The Storage and Retrieval of Aerometric Data (SAROAD) system which stores and reports information relating to ambient air quality.

3. The Hazardous and Trace Substance Emissions System (HATREMS) which stores and reports sources and emissions data for non-criteria pollutants.

4. The Source Test Data (SOTDAT) system which stores and retrieves relevant technical data collected during source emission measurements (i.e. stack tests).

5. The State Implementation Plans (SIPS) regulation system which provides retrievals of EPA-approved state air pollution control regulations.

6. The Emissions History Information System, which provides historical trends information on nationwide emissions, and may also function as a computerized technique for examination of air pollutant emissions scenarios.

$$
\mathrm{C}-2
$$




\section{AEROS, paǵe 2}

7. The Air Pollutant Emissions Report (APER) tracking system which keeps records of APER forms mailed to emissions sources required to report data to EPA.

8. The Weighted Sensitivity Analysis Program (WSAP) which operated on NEDS data to compute the er ror which can be tolerated in the emission estimate for each source category in order that some overall user-specified error shall not be exceeded.

9. The Source Inventory and Emission Factor Analysis (SIEFA) program, which complements WSAP by computing the actual (as opposed to allowable) error in emission esti.mates for each source category in NEDS due to errors in emission inventory techniques and source data.

10. The Computer Assisted Area Source Emissions (CAASE) gridding system which performs the calculations to apportion NEDS county emissions totals to sub-county, gridded areas.

11. The Regional Emissions Projection System (REPS) which may be used to make regional emission projections up to the year 2000 .

Assessment: none

Analyses Performed: unknown

Funding: Environmental Protection Agency

Data Availability: see OAQPS Guideline AEROS Manual Series

Vol. I AEROS Overview

EPA Report, 1975

Archive Medium: unknown

Contact: James H. Southland, Chief (919) 688-8146

Technical Development Section (MD-14)

National Air Data Branch

Office of Air Quality Planning and Standards

U.S. Environmental Protection Agency

Research Triangle Park

North Carolina 27711 
AEROS, page 3

Citations: 1.3.2--2.1--3.1.3; 1.3.2--2.2--3.1.3; 1.3.2--2.3--3.1.3;

$1.3 .2--2.3--3.3 .3 ; 1.3 .2--2.3--3.3 .4 .1$;

$1.3 .2--2.3--3.3 .5 ; \quad 1.3 .2--2.4--3.1 ; 1.3 .2--2.5--3.1$;

$1.3 .2--2.7--3.3 .5 ; \quad 1.3 .2--2.9--3.1 .3$ 
Data Base: ANCR-1213

Title: Geological Aspects of an Assessment of the National Potential for Non-electrical Utilization of Geothermal Resources

Date: 1975 June

Geographic Area: Western States; Arizona; California, Colorado; Idaho; Montana; Nevada; New Mexico; Oregon; Utah;

Washington; Wyoming

Geographic Coordinates: not applicable

FIPS Code: $04000,06000,08000,16000,30000,32000,35000,41000$, $49000,53000,56000$

Abstract: This report presents exhaustive data on the potential sources and uses of geothermal water particularly in the Western third of the United States. This data includes the currently known or expected location, quality, quantity, and temperatures of geothermal sources. Included are discussions of the more important sources. Recommendations a re made relative to potential utilization of these sources. In addition, suggestions are presented for the scientific evaluation of the se and other potential geothermal sources.

Assessment: none

Analyses Performed: contained in document

Funding: U.S. Energy Research and Development Administration/Aerojet Nuclear Company

Data Availability: unknown

Archive Medium: hard copy

Contact: K.M. Hollenbaugh and C.R. Nichols

Boise State University

Boise, Idaliú

Citations: $\quad 1.3 .2--2.2--3.1 .2$ 
Data Base: APSFBA

Title: Source Emissions Data: Air Pollution Source Emissions Data for the San Francisco Bay Area

Date: 1973

Geographic Area: Pacific Coast States; Callifornia; San Francisco Bay Area

Geographic Coordinates: not applicable

FIPS Code: 06041, 06097, 06055, 06095, 06013,06001, 06085, 06081,06075

Abstract: Data are available for emission rates of seven air pollutants in the San Franciso Bay Area. These data were prepared in conjunction with the Bay Area Air Pollution Control District (BAAPCD) for use in numerical air pollution studies.

As ses sment: none

Analyses Performed: LIRAQ User's Guide - Source Emissions Data, Donald Hardy and William Johnston, to be published, 1975.

Funding: unknown

Data Availability: unknown

Archive Medium: unknown

Contact: Donald M. Hardy (x5481) and William Johnston ( $\times 8811$ )

(415) 447-1100

G-Division

Law rence Live rmore Laboratory

Live rmore, CA 94550

Master Control No. 56061

Citations: $\quad$ 1.3.2--2.3--3.1.3 
Model: ARAC

Title: Atmospheric Release Advisory Capability

Date: 1975

Geographic Area: ERDA Nuclear facilities

Geographic Coordinates: unknown

FIPS Code: not applicable

Abstract: The chief purpose of ARAC is to provide responsible site officials with estimates of the effects of atnospheric releases of hazardous materials as rapidly and accurately as possible. ARAC would develop a series of advisories concerning emergency or routine atmospheric releases to assist the site in its planning. At the heart of the ARAC concept are the numerical models that provide real-time regional assessments based upon input data from the site. These models vary in complexity from a single-trajectory model to an interfaced set of advanced regional transport and diffusion models covering the distance range of $\sim 10$ to $100 \mathrm{~km}$. The models, combined with other state-of -the-art technology for dose conversion, data handling, and communication, permit a greatly improved but economical means for predicting the effects of releases of toxic materials of any sort. While the primary function of $A R A C$ is to assist a site in emergency response, there are other more routine uses intended for this service. Some examples are:

1. Calculate and maintain an inventory of radioactivity in the source.

2. Maintain an updated inventory of routine releases of materials and their concentration in the enviromment.

3. Calculate doses from routine operations.

4. Perform sensitivity studies to ascertain changes in pathway drives that determine the biological impact possible from changes in site operations and in site location for projected facilities.

Aseesoment: none

Analyses Performed: unknown 
ARAC, page 2 .

Funding: U.S. Energy Research and Development Administration

Model Availability: See: "Atmospheric Release Advisory Capability," Marvin H. Dickerson and Richard. C. Orphan, UCRL Preprint-77200, September 8, 1975.

Archive Medium: unknown

Contact: Marvin H. Dickerson (415) 447-1100 x 8811

Associate Division Leader Atmospheric and Geophysical Sciences

Lawrence Livermore Laboratory

P. O. Box 808

Livermore, California 94550

Citations: $\quad 1.3 .2--2.3--3.1 .1$ 
Data Base: BEIC

Title: Battelle Energy Information Center

Date: 1973 July 1

Geographic Area: not applicable

Geographic Coordinates: not applicable

FIPS Code: not applicable

Abstract: The objective of BEIC is to portray the total energy-information field. Its scope includes (1) all the principal current and potential energy sources - coal, oil, gas, fusion, fission, geothermal, solar, wind, ocean currents, hydrogen, oil shale, tar sands; (2) all aspects of complete energydelivery system from capture to utilization - exploration, mining, drilling, processing, refining, transporting, converting, distributing; and (3) public-policy aspects of eneirgy.

Assessment: none

Analyses Performed: unknown

Funding: Battelle Energy Program

Data Availability: through Battelle Columbus

A rchive Medium: unknown

Contact: Peter L. Hofmann (x3162) and Willard Gersbacher (x3437)

Battelle Columbus Labo ratories

(614) 299-3151

505 King Avenue'

Columbus, Ohio 43201

Master Control No. 56020

Citations: $1.3 .2--2.6--3.3 .5 ; 1.3 .2--2.6--3.4 .2$;

$1.3 .2--2 \cdot 7--3 \cdot 3 \cdot 4 \cdot 1$ 
Model: BESOM

Title: Brookhaven Energy System Optimization Model

Date: 1972

Geographic Area: national

Geographic Coordinates: not applicable

FIPS Code: ' not applicable

Abstract: This model is concerned with the substitution of different fuels at the level of disaggregated demand and supply. . In addition, it estimates the volume of each type of pollutant by the energy system. It is an optimization model using linear programming. The model provides a feasible path between $n=27$ exogenous supply categories and $m=22$ exogenous demand categories. The objective function is the minimized solution of the present cost of the possible paths. Three constraints must be satisfied: the level of each kind of demand, the possibility of each kind of supply, and the levels of the different pollutions.

Supply categories are considered as follows:

- 14 kinds of central station plants that produce electricity as an intermediate energy form: coal steam electric, coal combined cycle, oil steam electric, oil combined cycle, gas turbines, gas steam electric, LWR electric, LMFBR electric, HTGR electric, CTR electric, hydroelectric, geothermal, solar and pumped storage electric.

- decentralized electrical supply systems: total energy systems and fuel cells.

- general purpose fuels delivered directly to consumers: liquids (domestic, imported, and shale oil, liquefied coal; methanol), gases (natural, coal-derived and oil-derived gases; coal-derived and electrolytic hydrogen), solids (underground and stripmined coal)

For each supply category, the model needs the knowledge of:

- the supply constraint given in units of $10^{15} \mathrm{Btu}$

- the amount of energy that can be delivered by a particular supply category, limited either by the energy conversion capacity or by the quantity of available energy resources. 
BESOM, page 2

Demand categories are considered as follows:

- exogenous demands: space heat, heat pump, air conditioning, water heating, miscellaneous electricity at 3-5 different load factors, process heat (low, intermediate and high temperature) iron ore reduction, petrochemicals, air transport, rail, truck and bus, automobile, enrichment.

- endogenous demands: for resource conversion to synthetics or electricity, for pumped storage or synthetic fuel production; equations which account for the electrical sector load structures on a seasonal and weekly basis.

The model incorporates air pollutants and other wastes generated by energy conversion activities that are proportional to the amount of energy delivered: $\mathrm{CO}_{2}, \mathrm{CO}, \mathrm{SO}_{2}, \mathrm{NO}$, particulates, hydrocarbon, radioactive wastes and thermal wastes. Other pollutants and land use will be incorporated in the expanded model.

The coefficients of cost in the objective function reflect the necessary cost of the facilities in the energy supply system as well as fuel and other operating costs. The necessary cost of capital for the electric supply category is a function of the plant load factor which is also a function of each specific demand category.

Assessment: none

Analyses Performed: "Brookhaven Energy System Optimization Model," Ellen A. Cherniavsky, Brookhaven National Laboratory Report BNL-19569, 1974.

Funding: unknown

Model Availability: unknown

Archive Medium: unknown

Contact: Kenneth Hoffman (516) 345-2123 x 2443

Brookhaven National Laboratory

Upton, New York 11973

Citations: $1.3 .2--2.3--3.1 .3 ; 1.3 .2--2.6--3.3 .1 ; 1.3 .2--2.6--3.3 .5$;

$1.3 .2--2.6--3.4 .1 ; 1.3 .2--2.6--3.4 .2$ 
Data Base: BLM/DF-73-005

Title: Bureau of Land Management: Erosion Ratings

Date: unknown

Geographic Area: national by watershed

Geographic Coordinates: not applicable

FIPS Code: all

Abstract: Data collected from 2500 watersheds, classed by vegetation cover, type and root depth. Four erosion classes listed by acreage.

Assessment: none

Analyses Performen: unknown

Funding: Bureau of Land Management

Data Availability: Bureau of Land Management, Division of Data Processing, Denver Service Center, Bldg. 50, Denver, Colorado 80225, as AREM063, DREM064.

Archive Medium: 1 reel 7 -track, 556 bpi, odd parity magnetic tape, Burroughs B5500 internal code

Contact: Bureau of Land Management

Division of Data Processing

Denver Service Center

Bldg. 50

Denver, CO 80225

Master Control No. 60340

Citations: $1.3 .2--2.2--3 \cdot 1 \cdot 1 \cdot 4.2$ 
Data Base: BLS/DF-73-007

Title: Current Employment Statistiç Program

Date: 1939 to date

Geographic Area: National, states, areas (mostly SMSA's)

Geographic Coordinates: not applicable

FIPS Code: all

Abstract: The current employment statistics program generates state and area statistics as well as the national series. State agencies prepare state and area statistics monthly, selecting for publication those industries which best reflect currently significant economic activities. This file contains annual averages only for about 18,000 monthly time series of industry employment (all employees), and (1) average weekly earnings; (2) average weekly hours, and (3) average hourly earnings of production workers in states and selected areas (mostly SMSA's). There are approximately 325,000 variable length records. The format was originally designed for. IBM 7070 series computers.

Assessment: none

Analyses Performed: "Employment and Earnings" (monthly publication) "Monthly Labor Review" (monthly publication)

Funding: Bureau of Labor Statistics

Data Availability: National Technical Information Service Computer Products Office Springfield, VA 22151

Archive Medium: 6 reels of 7 -track, 556 bpi, BCD-code magnetic tape

Contact: Bureau of Labor Statistics

Wa shington, DC

Master Control No. 60471

Citations: $1.3 .2--2.6--3.4 .1$ 
Data Base: CDWR-143-7

Title: Geothermal Wastes and Water Resources of Salton Sea Area

Date: 1970 Eebruary

Geographic Area: Pacific Coast States; California; Imperial Valley

Geographic Coordinates: not applicable

FIPS Code: 06025

Abstract: not available

Assessment: none

Analyses Performed: California Department of Water Resources

Bulletin No. 143-7 (February 1970)

Funding:

Data Availability: Office of Procurement, Documents Section, P.O. Box 20191, Sacramento, CA, $\$ 3,50$

Archive Medium: journal article

Contact: S. L. Werner and L. J. Olson

Citations: $1.3 .2--2.2--3.1 .2$ 
Data Base: CES/HNL 1

Title: Census of Employment Survey

Data: 1970 census

Geographic Area: National

Geographic Coordinates: not applicable

FIPS Code:

Abstract: Data from the 1970 census of population and housing providing detailed social and economic data on persons residing in twenty-four of sixty-eight low income areas. CES public use computer tapes for the se areas were provided only when these areas had a population size of 250,000 or more to preserve confidentiality of the individual household and person records provided in this data.

Assessment: none

Analyses Performed: unknown

Funding: unknown

Data Availability: available through RUSTIC (q. v.)

Archive Medium: magnetic tape

Contact: Andrew S. Loebl or Deborah F. Burch (615) 483-8611,

Ext. 3-0311 or 3-0353

RUSTIC

Oak Ridge National Laboratory

P.O. Box X

Oak Ridge, TN 37830

Master Control No. 56251

Citations: $1.3 .2--2.8--3.4 .1$ 
Data Base: CES/HNL 2

Title: 1972 Census of Governments: Name and Addresses, Finance, Detailed Tax File and Employment File

Date: 197.2

Geographic Area: National, regions, states, counties, cities, independent school districts, selected special districts, municipalities and townships with populations of 10,000 or more

Geographic Coordinates: not applicable

FIPS Code: all

Abstract: A census of governments which is taken every 5 years (years ending in 2 and 7) identifies and lists all government units including school districts and special districts such as housing authorities and bridge commissions, as well as generalpurpose governments, such as counties, municipalities, and townships. It also produces statistics concerning the numbers and characteristics of governments in the United States, elective officials, value of taxable property, public employees and payrolls, and governmental revenues, expenditures; debt, and financial assets. Annual surveys provide statistics on governmental finances and on public employment. These reports present figures for federal, state, and city governments. Quarterly reports provide data on tax revenue, holdings of selected public-employment retirement systems, and construction expenditures of state and local governments. The annual governmental, finance surveys cover governments' fiscal years, and deal, with revenue by source (including tax revenue by type of tax), expenditure by function and by character and object, indebtedness and debt transactions, and financial assets. Differing degrees of detail are published for various levels: more than 300 categories on finances of the individual state governments, nearly 200 categories for national estimates of local government finances, and about 40 categories for state-by-state data covering both state and local government finances. The annual sample survey of public employment covers the month of October, and deals with numbers of employees (full-time, part-time, and full-time equivalent) and October payrolls classified in terms of about 25 functional categories. 
CES/HNL 2 page 2

Assessment: none

Analyses Performed: unknown

Funding: unknown

Data Availability: available through RUSTIC (q. v.)

Archive Medium: magnetic tape

Contact: Andrew S. Loebl or Deborah F. Burch (615) 483-8611, Ext. 3-0311 or 3-0353

RUSTIC

Oak Ridge National Laboratory

P.O. Box X

Oak Ridge, TN 37830

Master Control No. 56249

Citations: $1.3 .2--2.6--3.4 .1 ; 1.3 .2--2.8--3.3 .5$;

$1.3 .2--2.8--3.4 .1$ 
Data Base: CES/HNL 3

Title: U.S. Census, 1970, Summary Tapes, Fifth Count, Enumeration District/Block Group File

Date: 1970

Geographic Area: U.S. Enumeration Districts and Block Groups

Geographic Coordinates: not applicable

FIPS Code: all

Abstract: Data are included on population characteristics such as: income, race, age, sex, education, employment, poverty status; and data on housing characteristics such as value, rent, structural characteristics and facilities. This data file is the only source for 1970 census sample information for geographic entities below the cuunly level and for placco below 2500 in population.

Assessment: none

Analyses Performed: unknown

Funding: U.S. Bureau of the Census

Data Availability: available through RUSTIC (q.v.)

Archive Medium: magnetic tape

Contact: Andrew S. Loebl or Deborah F. Burch (615) 483-8611,

Ext. 3-0311 or 3-0353

RUSTIC

Oak Ridge National Laboratory

P.O. Box X

Oak Ridge, TN 37830

Master Control No. 56242

Citations: $1.3 .2--2.6--3.4 .1$ 
Model: CLEL

Tiele: California Loss - Economic Loss to Single Family Dwellings in California by Census Tract and Earthquake Intensity

Date: $1973-1976$

Geographic Area: Pacific Coast States; California

Geographic Coordinates: not applicable

FIPS Code: 06001 through 06115

Abstract: The model uses empirically determined damage factors and damage ratios to develop a relationship between earthquake intensity at a site and resulting damage to single family dwellings and a relationship between the replacement cost value of the damaged dwellings and the repair cost of the damage. For a given earthquake, it is then possible to determine the loss, the number of units damaged and a percentage deductible loss.

Assessment: none

Analyses Performed: Unpublished report to the Department of Housing and Urban Development

Funding: Department of Housing and Urban Development and U.S. Geological Survey

Model Availability: unknown

Archive Medium: unknown

Contact: W.A. Rinchart (303) 234-4041

Branch of Seismicity and Risk Analysis

U.S. Geological Survey

Denver Federal Center (D-1)

Denver, Colorado 80225

Master Control No. 56273

Citations: 1.3.2--2.8--3.1.1.4.5; 1.3.2--2.9--3.1.1.4.5 
Data Base: COM/DF-73-008

Title: Geophysical and Solar-Terrestrial Data

Date: unknown

Geographic Area: global

Geographic Coordinates: not applicable

FIPS Code: not applicable

Abstract: The National Geophysical and Solar-terrestrial Data Center (NGSDC) acquires, processes, analyzes and disseminates. solid earth and solar-terrestrial environmental data from worldwide sources. It attempts to meet user requirements and to provide the use $r$ with ready access to needed information. In addition, it provides facilities for World Data Centers in its subject fields. The scope of NGSDC coverage is geomagnetism; seismology; gravity, bathymetry; marine geology; aeronomy; space related geophysical data from land, marine, and airborne platforms. Source documents: geophysical observations from data from ships, observatories, selected space vehicles, surveys and other data generators on a worldwide basis.

Assessment: none

Analyses Performed: unknown

Funding: National Oceanic and Atmospheric Administration

Data Availability: Available through NGSDC

Archive Medium: unknown, output formats variable

Contact: National Geophysical and Solar-Terrestrial Data Center 30th and Marine Streets

Boulder, Colorado 80302

(303) $499-1000$

Master Control No. 60336

Citations: $1.3 .2--2.9--3.1 .1 .4 .5$ 
Data Base: COM/DF-73-009

Title: National Climatic Center Data Base

Date: unknown

Geographic Area: global

Geographic Coordinates: not applicable

FIPS Code: not applicable

Abstract: The National Climatic Center (NCC) collects, evaluates, publishes, and distributes climatological data. It archives and microfilms the basic records and retrieves them as needed. The NCC is the collection center and custodian of most United States weather records and is the largest data center in the Environmental Data Service. The World Data Center A for meteorology (and nuclear radiation) is housed with the NCC at Asheville, North Carolina. The scope of NCC coverage is global (surface to satellite orbit elevations). Meteorological elements include clouds, temperature, humidity, pressure, visibility, wind direction, wind speed, precipitation, and solar radiation. Source documents: a wide variety of digitized documents, each containing selected meteorological measurements.

Assessment: none

Analyses Performed: unknown

Funding: National Oceanic and Atmospheric Administration

Data Aväiläbility: NCC helps users solve specific climatological problems by furnishing data in the particular form and quantity needed, preparing special tabulations or summaries on request, and providing referrals to private meteorological consultants for those who require assistance in interpreting the information supplied.

Archive Medium: Microfilm and 7-track, 200/556/800 bpi, even parity, BCD or 9-track, 800 bpi, odd parity, EBCDIC magnetic tape. 
COM/DF-73-009, page 2

Contact: National Climatic Center (704) 254-0961 x 203

National Oceanic and Atmospheric Administration

Federal Building

Asheville, N.C. 28801

Master Control No. 60333

Citations: $1.3 .2--2.3--3.1 .3$ 
Data Base: COM/DF-73-022

Title: Economic Development Administration Quick Query Service

Date: unknown

Geographic Area: national at census tract level

Geographic Coordinates: not applicable

FIPS Code: all

Abstract: Quick Query is a service intended to allow the users to identify, select, accumulate, categorize and receive required data in an effective readable format without having to acquire their own basic data files and related retrieval system. The Composite Mapping System (CMS) is a computerized capability for merging coincident sets of digitized map data into differentially weighted combinations or composite maps. The system stores data at any scale in a $120 \times 120$ array of grid cells. As used by EDA, each grid cell corresponds to an area of 2 minutes of latitude by 2 minutes of longitude or about 4 square miles. This scale (about 1:1, 167,000) is convenient for performing multistate regional analyses. From this design scale, scale reductions of $\frac{1}{2}, 1 / 3, \frac{1}{4}$, etc. are possible. CMS can produce single factor maps in digital printout form of two types - free form or maps based on governmental or jurisdictional units at which the data are collected. The free form maps can represent: zones of accessibility to linear or point phenomena such as transportation or power networks and nodes; isopleth maps of continuously variable phenomena such as climate; or locational maps depicting forest types or land use. Multiple factor maps are produced as differentially weighted combinations of up to 120 different free form and governmental unito mapo of the same geographical area at the same map scale.

CMS may be used through the Quick Query service in conjunction with any of the following data files: 1970 - census data files, county merge, income file (1929, 1940, 1950, 1962, 1966-70), or county business patterns files (source: census).

Assessment: none

Analyses Performed: unknown 
$\mathrm{COM} / \mathrm{DF}-73-022$, page 2

Funding: Economic Development Administration

Data Availability: National Technical Information Service

Computer Producțs Office.

Springfield, VA 22151

Archive Medium: magnetic tape

Contact: Economic Development Administration (202) 377-2000

Department of Commerce.

Washington, D. C.

Master Control No. 60514

Citations: 1.3.2--2.6--3.1.1.2; 1.3.2--2.6--3.1.3;

$1.3 .2--2.6--3.4 .2 ; 1.3 .2--2.8--3.3 .1$ 
Data Base: CRIB

Title: Computerized Resources Information Bank

Date: 1970

Geographic Area: worldwide

Geographic Coordinates: not applicable

FIPS Code: not applicable

Abstract: CRIB provides a rapid means for organizing and summarizing information on mineral resources and for displaying the results. CRIB consists of a set of variable-length records containing the basic information needed to characterize one or more mineral commodities, a mineral deposit, or several related deposits. The information consists of text, numeric data, and codes. Some topics covered are: name, location, commodity information, geology, production reserves, potential resources, and réferences.

The data are processed by the GIPSY program, which per forms all the processing tasks needed to build, operate, and maintain the CRIB file. The sophisticated retrieval program allows the user to make highly selective searches of the file for words, parts of words, phrases, numeric data, word ranges; numeric ranges, and others, and to interrelate variables by logic statements to any degree of refinement desired. Three print options are available, or the retrieved data can be passed to another program for further processing.

Assessment: none

Analyses Performed: Uses of CRIB - A GIPSY - formatted Mineral Resources Computer File, James A. Calkins and Linda E. Deiter, U.S. Geological Survey Report.

Funding: U.S. Geological Survey

Data Availability: unknown

Archive Medium: unknown 
CRIB, page 2

Contact: James A. Calkins (703) 860-6604.

George Mason (703) 860-7570

U. S. Geological Survey

Reston, VA 22092

Master Control No. 56264

Citations: $1.3 .2--2.6--3.1 .1 .2$ 


\section{Model: CSU/HUNT}

Title: Simulation Modeling, Grassland Ecology

Date: unknown

Geographic Areà: not applicable

Geographic Coordinates: not applicable

FIPS Code: not applicable

Abstract: A systems approach to problems in basic and applied grassland ecology, interdisciplinary with botanists, zoologists, soil scientists, meteorologists.

Assessment: none

Analyses performed: unknown

Funding: unknown

Model Availability: unknown

Archive Medium: unknown

Contact: Hunt, H.W.

Natural Resources Ecology Laboratory

Colorado State University

Ft. Collins, Colorado 80523

Citations: $1.3 .2--2.4--3.2 .1 ; 1.3 .2--2.4--3.2 .2$ 
Data Base: DOCBOC 10

Title: Census of Governments

Date: 1974 September 25

Geographic Area: national

Geographic Coordinates: not applicable

FIPS Code: not applicable

Abstract: A compilation of governmental organization, taxable property values, public employment, and government finances data.

Assessment: none

Analyses Performed: unknown

Funding: Bureau of the Census

Data Availability: see contact

Archive Medium: unknown

Contact: Sherman Landau (301) 763-7366

Chief, Governments Division

Bureau of the Census

Department of Commerce

Suitland, Maryland

Master Control No. 70062

Citations: $1.3 .2--2.6--3.4 .1$ 


\section{Data Base: DOCBOC 11}

Title: Annual Surveys of Governmental Finance and Public Employment:

Date: 1974 September 25

Geographic Area: national

Geographic Coordinates: not applicable

FIPS Code: not applicable

Abstract: Government finance and public employment data for intercensal years.

Assessment: none

Analyses Performed: unknown

Funding: Bureau of the Census

Data Availability: see contact

Archive Medium: unknown

Contact: Sherman Landau (301) 763-7366

Chief, Governments Division

Bureau of the Census

Department of Commerce

Suitland, Maryland

Master Control No. 70063

Citations: $1.3 .2--2.6--3.4 .2$ 
Data Base: DOI/BLM

Title: Bureau of Land Management:' Preparation of Information System for Energy Alternatives

Date: 1974 October 17

Geographic Area: national; state; local

Geographic Coordinates: not applicable

FIPS Code: all

Abstract: To provide data for energy and/or mineral oriented projects, facilitate preparation of environmental reports and impact statements and management analysis, facilitate general or specific analysis of energy alternatives, and store historical upland and OCS tract leasing data:

Assessment: none

Analysès Performed: unknown

Funding: Bureau of Land Management, Department of the Interior

- Data Availability: unknown

Archive Medium: unknown

Contact: Gretchen E. Tatting (202) 343-8403

Chief, Minerals Data Reference Center

Bureau of Land Management

Department of the Interior

Washington, D.C.

Master Control No. 70114

Citations: $1.3,2--2.6--3.4$ 
Data Base: DOI/DF-73-001

Title: Nation-wide Outdoor Recreation Inventory

Date: 1965

Geographic Area: national by state; county, and city

Geographic Coordinates: not applicable

FIPS Code: all

Abstract: A 1965 nationwide area and agency inventory of public outdoor recreation supply covers federal, state, county, and city jurisdictions. It includes acreage, facilities, and visitation data. (Cities under 50, 000 population were sampled.)

Assessment: none

Analyses Performed: unknown

Funding: Bureau of Outdoor Recreation

Data Availability: Division of Planning and Research, Bureau of Outdoor Recreation, Department of the Interior, Washington, D. C. 20240

Archive Medium: Two reels of 9-track, 800 bpi, odd parity, EBCDIC magnetic tape.

Contact: Bureau of Outdoor Recreation (202) 343-5726

Department of the Interior

Washington, D. C. 20240

Master Control No. 60155

Citations: $1.3 .2--2.5--3.3 .1 ; 1.3 .2--2.5--3.3 .2$;

$1.3 .2--2.8--3.3 .2$ 
Data Base: DOI/EIS-1

Title: Final Environmental Statement for the Geothermal Leasing Program. Vol.I. Promulgation of Leasing and Operating Regulations for the Geothermal Leasing Program

Date: 1973

Geographic Area: Western States; Alaska; California; Idaho; Montana; Nevada; New Mexico; Oregon; Utah; Washington

Geographic Coordinates: not applicable

FIPS Code: $02000,06000,16000,30000,32000,35000,41000,49000$, 53000

Abstract: Volume I of this statement primarily relates to the promulgation of leasing and operating regulations for implementation of the geothermal leasing program as authorized by the Geothermal Steam Act of 1970 (30 USC 1001-1025 (1970). The proposal is described in Chapter I. The national energy situation, geothermal energy resources and their potentials, description of the environmental setting of the western states are included in Chapter II. The promulgation of leasing and operating regulations, the environmental impacts of the proposed action, mitigating measures, adverse impacts which cannot be avoided, the relationships between local short-term uses of man's. environment and maintenance and enhancement of long-term productivity, and irreversible and irretrievable commitments of resources are discussed in detail in Chapter III. Chapter IV includes discussions of alternatives for timing of actions, environmental provisions of regulations, leasing options, Federal and private exploration and development and electrical energy sources.

Asses sment: none

Analyses Performed: contained in document

Funding: U.S. Department of the Interior

Data Availability: Superintendent of Documents, Stock No. 2400-00789, $\$ 4.20$

Archive Medium: hard copy 
DOI/EIS-1, page 2

Contact: Office of the Secretary (20.2) 343-1100

U. S. Department of the Interior

Washington, DC 20240

Citations: $1.3 .2--2 \cdot 5---3 \cdot 3.5$ 
Data Base: DOI/EIS-2

Title: Final Environmental Statement for the Geothermal Leasing Program, Vol. II. Clear Lake - Geysers KGRA, Mono-Long Valley KGRA; Imperial Valley KGRA...

Geographic Coordinates: not applicable

FIPS Code: 06033, 06097, 06051, 06025

Abstract: Volume II primarily covers the environmental evaluations for the three areas in California being considered for leasing (Clear Lake-Geysers, Mono Lake-Long. Valley and Imperial Valley). These statements generally follow the same format as used in Volume I but with particular emphasis on environmental considerations pertaining to each area. General environmental information as contained in Volume $I$ is not repeated in the individual statements unless such is needed. to facilitate the description of actions, mitigating measures and environmental impacts. Also included in this volume a re summaries of comments and Departmental responses relative to the Draft Environmental Impact Statement; description of the consultation and coordination involved in the development of the proposal and the Draft Statement, and coordination in the review of the Draft Statement. Large scale maps of the three proposed lease areas are included in the pocket at the end of this volume.

Asses sment: none

Analyses Performed: contained in document

Funding: U.S. Department of the Interior

Data Availability: Superintendent of Documents, Stock No. 2400-00790, $\$ 5.85$.

Archive Medium: hard copy

Contact: Office of the Secretary (202) 343-1100

U.S. Department of the Interior

Washington, D. C. 20240

Citations: $1.3 .2--2 \cdot 5--3.3 .5$ 
Data Base: DOI/EIS -4

Title: Final Environmental Statement for the Geothermal Leasing Program. Vol. IV. Comments received ...

Date: 1973

Geographic Area: not applicable

Geographic Coordinates: not applicable

FIPS Code: not applicable

Abstract: Volume IV includes copies of all comments received on the Draft Environmental Impact Statement and the proposed regulations of July 23, 1971 and May 3, 1972. Also included in this volume are copies of transcripts of the hearings held at Reno, Nevada on November 9, 1971; Sacramento, California on November 11, 1971; and Portland, Oregon on November 12, 1971 .

Assessment: none

Analyses Performed: not applicable

Funding: not applicable

Data Availability: Superintendent of Documents, Stock No. 2400-00788, $\$ 5.65$.

Archive Medium: hard copy

Contact: Office of the Secretary (202) 343-1100 U.S. Department of the Interior Washington, DC 20240

Citations: $1.3 .2--2 \cdot 5--3 \cdot 3 \cdot 5$ 
Model: DRI

Title: Data Resources Incorporated Energy Model

Date: 1975

Geographic Area: national by thirteen regions

Geographic Coordinates: not applicable

FIPS Code: not applicable

Abstract: Investigates the interrelationships between energy utilization and economic activity. Detailed projections of National and Regional economic growth are translated into a projections of energy requirements by region and by source in order that alternative economic scenarios and energy policies can be evaluated. All U.S. oconomic activities are taken into account by using a very large economic model of the U.S. economy and an associated econometric model of the regional energy demands, supplies and movements. Special emphasis is placed on the relationships between technologies (autos, houses, etc.) energy prices and economic activity in forecasting demand. Energy sectors are residential, commercial, industrial, utility, and transportation. Special emphasis is placed on energy sectors, and sensitivity to different governmental policies is tested. Only foreign and government sectors are exogeneous; all others (mainly business and household) are endogeneous.

Assessment: none

Analyses Performed: unknown

Funding: unknown

Model Availability: unknown

Archive Medium: unknown

Contact: Philip K. Verleger, Jr. Data Resources

Lexington, Massachusetts

Citations: $1.3 .2--2.6--3.3 .5$

$$
\text { C }-36
$$


Model: EAM/CIM

Title: Regional Energy Policy Model

Date: 1973

Geographic Area: national

Geographic Coordinates: not applicable

FIPS Code: not applicable

Abstract: Examines the supply, demand and price of primary energy sources and electricity subject to as sumptions about future policies and technology changes.

The type of factors that can be examined are:

- New technology

- Environmental restrictions

- Import quotas

- Energy conservation measures

- Rationing

- Regulations or taxes that force substitution

- Price increase

The system is composed of 4 primary energy sources (coal, oil, natural gas, nuclear) and 4 end-use sectors: industrial, electric utilities, transportation, residential/commercial.

Assessment: none

Analyses Performed: unknown

Funding: Westinghouse Electric Company

Model Availability: unknown

Archive Medium: unknown

Contact: P.F. Schweizer, C.G. Love, or J. Hunter Chiles Westinghouse Electric Company

Pittsburgh; Pennsylvania

Citations: $1.3 .2--2.6--3.3 .5 ; 1.3 .2--2.6--3.4 .2$ 


\section{Model: EEDF}

Title: Electric Energy and Demand Forecasting

Date: 1976

Geographic Area: regional

Geographic Coordinates: not applicable

FIPS Code: not applicable

Abstract: Forecasts electrical energy and demand for regions through the use of a model that interrelates growth of the regional economy and population to the power demand. Goal is to provide detailed forecasts in order to facilitate analysis of causes of change.

The model develops, for the region being analyzed, detailed forecasts of household formation, employment, income, housing, energy, and peak demand. Special emphasis is placed on describing the hourly use patterns which is used to directly forecast demand and energy. Residential demand is modeled in terms of appliance, heating, cooling, and lighting loads for household income classes. Commercial load is forecast by type of commercial establishment and by lighting and equipment load, space heating, and cooling. Industrial demand is forecast by 2 -digit SIC classification.

Assessment: none

Analyses Performed: unknown

Funding: unknown

Model Availability: unknown

Archive Medium: unknown

Contact: H.R. Hamilton, J.M. Griffin, J.B. Broehl, or G. A. Juras Battelle Memorial Institute

Columbus, Ohio

Citations: $1.3 .2--2.6--3.4 .1$ 
Model: EEIED

Title: Environmental and Economic Inputs and Energy Development

Date: 1975

Geographic Area: Rocky Mountain States; Upper Colorado River Basin; Colorado

Geographic Coordinates: not applicable

FIPS Code: 08000

Abstract: Analyzes the economic, hydrologic, and air quality implications of different patterns of energy resource development in the Upper Colorado River Basin.

This system integrates three input-output models of approximately 30 sectors covering the Green River Basin, the Colorado River Upper Main Stem Basin, and the San Juan Basin with a monthly hydrologic model and a point source air pollution diffusion model. Any proposed pattern of energy industry development can be fed into the model either as a change in export pattern or as a change in regional economic structure or both.

Assessment: none

Analyses Performed: unknown

Funding: unknown

Model Availability: unknown

Archive Medium: unknown

Contact: C. Howe, J. Kreider, or B. Udis Department of Economics University of Colorado Boulder, Colorado

Citation: $1.3 .2--2.2--3.1 .2 ; 1.3 .2--2.3--3.1 .3 ; 1.3 .2--2.6--3.4 .1$ 
Model: EEPM

Title: Energy and Environmental Policy Model

Date: 1976

Geographic Area: any region

Geographic Coordinates: not applicable

Abstract: Simulates the responses of utility and industrial sources to air pollution control and/or energy policies. Presently only. sulfur dioxide and particulate control regulations are modeled. Effects of changes in energy prices can also be simulated.

The model calculates the allowable emissions associated with a control strategy, compares this to existing emissions, calculates the required emissions reduction, and selects a least cost control option. This is done on an individual source basis. A library of control strategies is endogenous to the program. The control options available to the individual pollution sources are the installation of control equipment and fuel substitution.

Assessment: none

Analyses Performed: unknown

Funding: National Science Foundation (RANN)

Model Availability: unknown

Archive Medium: unknown

Contact: Alan S. Cohen or G.A. Concaildi (312) 739-7711 x 4311

Argonne National Laboratory

9700 S. Cass Avenue

Argonne, Illinois 60439

Citations: $1.3 .2--2.3--3.3 .5 ; 1.3 .2--2.6--3.1 .3 ; 1.3 .2--2.6--3.4 .2$ 
Data Base: EMDB

Title: Energy Model Data Base

Date: 1975

Geographic Area: national

Geographic Coordinates: not applicable

FIPS Code: not applicable

Abstract: Contains MERES (Matrix of Environmental Residuals for Energy Systems) data on particulates; air and water pollutants, solid waste, land use, occupational health effects classed by energy source - - coal and its derivatives, oil, natural gas, fission, with solar and geothermal sources. being added.

Assessment: none

Analyses Performed: "Energy Alternatives: A Comparative Analysis" Science and Public Policy Program, University of Oklahoma, May 1975

Funding: National Science Foundation, Council on Environmental Quality

Data Availability: see contact

Archive medium: unknown

Contact: Murray Goldberg

Energy Systems Analysis Group:

Brookhaven National Laboratory

Upton, New York 11973

Citations: 1.3.2--2.2--3.1.2; 1.3.2--2.2--3.1.3; 1.3.2--2.5--3.3.1;

$1.3 .2--2.7--3.3 .4 ; 1.3 .2--2.8--3.3 .1 ; 1.3 .2--2.8--3.4 .2$ 
Data Base: EMPP

Title: Employment Projection Project

Date: 1973

Geographic Area: national, states, standard metropolitan statistical areas.

FIPS Code: all states, SMSA's

Abstract: Summary of the source data used: national employment by 579 industries for the years $1958-1972$ with a projection to 1980. 'Local' employment by industry (number and type of industries may vary with the area) for most states plus many SMSA's over 25, 000 for the years 1958-1972 maximum (data may not exist for all 15 years). National employment 'matrices' (for 422 occupations within each of 201 industries) for 1970 and. 1980. 'Local' employment (for 201 industries) for 1970 and 1980 for most states plus many SMSA's over. 250,000. Rates of separation from the labor force (due to death, retirement or other withdrawals for 422 occupations) within each state plus the District of Columbia.

Assessment: none

Analyses Performed: Employment Projection Project, Brad Heckman, Special "BARB" output, 26 June 1974.

Funding: Manpower Administration of the Department of Labor

Data Availability: available through SEEDIS (q. v.)

Archive Medium: unknown

Contact: Brad Heckman (415) 843-2740, ext. 6068

Mathematics and Computing Group

Lawrence Berkeley Laboratory

Berkeley, CA 94720

Master Control No. 56126

Citations: $1.3 .2--2.6--3.4 .1$ 
Data Base: ENDEX

Title: Environmental Data Index

Date: 1975

Geographic Area: Atlantic Coast States; Great Lakes States; Gulf States; Pacific Coast States; New Hampshire, Massachusetts; New York; New Jersey; Delaware; Maryland; Virginia; North Carolina; South Carolina; Georgia; Florida; Alabama; Mississippi; Louisiana; Texas; California; Oregon; Washington; Wisconsin; Minnesota; Michigan; Ohio; Illinois; Indiana; Pennsylvania

Geographic Coordinates: not applicable

FIPS Code: 01000, 06000, 12000, 13000, 17000, 18000, 22000, 24000, $25000,26000,27000,28000,33000,34000,36000,37000$, $39000,41000,42000,45000,48000,51000,53000,55000$

Abstract: Referral file only, for aeronomy, cartography, climatology, coastal zone management, environmental engineering, geodesy, hydrography, hydrology, marine geology, meteorology, oceanography, space, solar science

Assessment: none

Analyses Performed: not applicable

Funding: National Oceanic and Atmospheric Administration

Data Availability: ENDEX services and products include

- Access to specialized indexes of environmental data, grouped by geographic areas, institutions, or disciplines.

- On-line, interactive searches of the indexes to answer specific questions concerning the availability and whereabouts of data files.

- A quick response determination of the costs of retrieval from large data files.

- Data catalogs from large NOAA environmental data collection projects.

Archive Medium: unknown 


\section{ENDEX page 2}

\section{Contact: Environmental Data Services at:}

National Climatic Center

National Oceanic and Atmospheric Administration

Federal Building

A sheville, N.C. 28801

Telephone: (704) 258-2850 Ext. 683

Federal Telecommunications System

(FTS): (704) 254-0683

National Geophysical and Solar-Terrestrial Data Center

National Oceanic and Atmospheric Administration

Boulder, Colo. 80302

Telephone: (303) 499-1000, Ext. 6323

- Federal Telecommunications System

(FTS): (303) 499-6323

Wa shington, DC 20235

Telephone: (202)634-7381

Citations: 1..3.2--2.1--3.1; 1.3.2--2.2--3.1.2; 1.3.2--2.2--3.2.1;

$1.3 .2--2.2--3.2 .2 ; 1.3 .2--2.2--3.3 .1 ; 1.3 .2--2.2--3.3 .2$;

$1.3 .2--2.2--3.3 .3 ; 1.3 .2--2.2---3.3 .4 .1 ; 1.3 .2--2.3--3.1 .3$;

$1.3 .2--2.3--3.2 .1 ; 1.3 .2--2.3--3.2 .2 ; 1.3 .2--2.3--3.3 .1$;

1.3.2--2.3--3.3.2; 1.3.2--2.3--3.3.3; 1.3.2--2.3--3.3.4.1;

$1.3 .2--2.3--3.3 .4 .2 ; 1.3 .2--2.4--3.2 .1 ; 1.3 .2--2.4--3.2 .2$;

$1.3 .2--2.5--3.1$ 
Data Base: ERDA/EA/TVL

Title: An Environmental Assessment of Proposed Goethermal Well Testing in the Tigre Lagoon Oil Field, Vermilion Parish, Louisiana

Date: 1976 March

Geographic Area: Gulf Coast States; Louisana; Vermilion Parish

Geographic Coordinates: $30^{\circ} \mathrm{N}$. Lat.; $92^{\circ} \mathrm{W}$. Long.

FIPS Code: 22113

Abstract: This report is an environmental assessment of the proposed testing of two geopressured, geothermal aquifers in central coastal Louisiana. The testing will be done by Osborn, Hodges, and Roberts Engineering, Lafayette, La.; under contract with the Energy Research and Development Administration (ERDA). On the basis of an analysis of the environmental setting, subsurface characteristics, and the proposed action, potential environmental impacts are determined and evaluated together with potential conflicts with federal, state, and local programs.

Assessment: none

Analyses Performed: contained in document

Funding: U.S. Energy Research and Development Administration

Data Availability: ERDA

Division of Geothermal Energy

Washington, DC 20545

Archive Medium: hard copy

Contact: Coastal Environments, Inc.

1260 Main Street

Baton Rouge, Louisiana 70802

Citations: $1.3 \cdot 2--2 \cdot 5--3 \cdot 3 \cdot 5$ 
Data Base: ERDA.02533

Title: Source Fluịds for Salton Sea Geothermal System

Date: 1969 February

Geographic Area: Pacific Coast States; California; Imperial Valley

Geographic Coordinates: not applicable

FIPS Code: 06025

Abstract: not available

Assessment: none

Analyses Performed: American Journal of Science 267, 249-255 (1969)

Funding:

Data Availability:

Archive Medium: journal article

Contact: H. Craig

Citations: $1.3 \cdot 2--2.2-3 \cdot 1 \cdot 1 \cdot 5 \cdot 1$ 
Data Base: ERDA 02821

Title: Terms, Conditions, Standards, and Application Procedures for Initial Geothermal Development: Imperial County, California

Date: 1973 May 10-11

Geographic Area: Pacific Coast States; California; Imperial Valley

Geographic Coordinates: not applicable

FIPS Code: 06025

Abstract: not available

Assessment: none

Analyses Performed: Proceedings of the National Conference on Geothermal Energy, May 10-11, 1973, Palm Springs, California, Vol. II pp 263-282.

Funding :

Data Availability:

Archive Medium: conference proceedings

Contact:

Citations: $1.3 .2--2.6--3.3 .5$ 
Data Base: ERDA 02837

Title: Geothermal Resources - Legal and Tax Considerations

Date: 1974 Feburary

Geographic Area: not applicable

Geographic Coordinates: not applicable

FIPS Code: not applicable

Abstract: Review of legal opinions on the ownership of geothermal resources. 38 citations.

Assessment: none

Analyses Performed: Geothermal Energy Magazine 2, 21-24 (April 1974).

Funding: Chevron Oil Company

Data Availability: in article

Archive medium: journal article

Contact: Willard A. Burton, Jr.

Manager of Contracts and Titles

Chevron Oil Company

Denver, Colorado

Citations: $1.3 .2--2 \cdot 6--3 \cdot 3 \cdot 5$ 
Model: ERDA 02877

Title: Assessment of $\mathrm{SO}_{2}$ Control Alternatives and Implementation Patterns for the Electric Utility Industry

Date: 1973 March

Geographic Area: not applicable

Geographic Coordinates: not applicable

FIPS Code: not applicable

Abstract: not available

Assessment: none

Analyses Performed: PB-224119-8-GA; HIT-550

Funding: Office of Science and Technology contract OST -40

Data Availability: unknown

Archive Medium: hard copy

Contact: Hittman Associates, Inc.

Columbia, Maryland

Citations: $1.3 .2--2.3--3.1 .3$ 
Data Base: ERDA 02892

Title: Limits of Microbial Existence: Temperature and $\mathrm{pH}$

Date: 1970 September 25

Geographic Area: United States; Wyoming; Yellowstone National Park;

California; Lassen Volcanic National Park; The

Geysers; Nevada; Steamboat Springs; Beowawe;

New Zealand; Iceland; Japan

Geographic Coordinates: not applicable

FIPS Code: $56029,06035,06097,32011,32031$

Abstract: A microscopic survey made to detect the presence of bacteria in hot springs of varying temperature and $\mathrm{pH}$ characteristics revealed that in neutral and alkaline hot springs bacteria a re found at temperatures up to the boiling point of water $192^{\circ}$ to $1000 \mathrm{C}$ depending on the altitude). In hot springs of increasing acidity the upper temperature limit at which bacterial are found decreases; at $\mathrm{pH} 2$ to 3 the upper temperature limit is $75^{\circ}$ to $800 \mathrm{C}$. Bacteria have thus been able to evolve with the ability to grow at either high temperature or high acidity, but not at both high temperature and high acidity. These results suggest that there are physicochemical limitations of the environment beyond which life is impossible.

Assessment: none

Analyses Performed: Science 169, $1316-1318$ (1970)

Funding: NSF. grant GB-7815; AEC contract COO-1804-16; Federal Water Pollution Control Administration

Data Availability: unknown

Archive Medium: journal article

Contact: Thomas D. Brock and Gary K. Darland Department of Microbiology Indiana University Bloomington, Indiana 47401

Citations: $\quad 1.3 .2--2.2--3.2 .2$ 
Model: ERDA 02893

Title: Man-made Earthquakes -- Way to Understand Natural Seismic Activity

Date: 1970

Geographic Area: not applicable

Geographic Coordinates: not applicable

FIPS Code: not applicable

Abstract: not available

Assessment: none

Analyses Performed: Geol. Rundsch. 59, 792-805 (1970)

Funding:

Data Availability:

Archive Medium: journal article

Contact: S. Mueller

Citations: $1.3 .2--2.9--3.1 .1 .4 .5$ 
Data Base: ERDA 02895

Title: Spectral Characteristics of Central Nevada Microearthquakes

Date: 1970 October

Geographic Area: Western States; Nevada

Geographic Coordinates: unknown

FIPS Code: 32000

Abstract: Fourier amplitude spectra were computed for 40 central Nevada microearthquakes, selected to consider, independently, effects of azimuth and distance from known sources. Spectral characteristics did not behave systematically as a function of azimuth from the recording site to the source, but peak spectral frequency was found to correlate strongly with event magnitude and to some degree also with focal distance. These preliminary results suggest that recordings of small earthquakes and microearthquakes can be used to provide detailed information on the character of seismic signals related to properties of the source and propagation path.

Assessment: none

Analyses Performed: Bulletin of the Seismological Society of America $60,1547-1559$ (1970)

Funding: Air Force Office of Scientific Research grants AFOSR-646-66, AFOSR-68-1564; National Science Foundation grant GP-5034

Data Availability: unknown

Archive Medium: journal article

Contact: Bruce M. Douglas and Ray Williams

Dept. of Civil Engineering

Alan Ryall

Mackay School of Mines Seismological Laboratory

University of Nevada

Reno, Nevada 89507

Citations: $1.3 \cdot 2--2.9--3.1 .1 .4 .5$

$$
\text { C }-52
$$


Data Base: ERDA 02900

Title: Complexity of Energy Release During the Imperial Valley, California, Earthquake of 1940

Date: 1970 February

Geographic Area: Pacific Coast States; California; Imperial Valley

Geographic Coordinates: not applicable

FIPS Code: 06025

Abstract: The pattern of energy release during the Imperial Valley, California, earthquake of 1940 is studied by analyzing the El Centro strong motion seismograph record and records from the Tinemaha seismograph station, $546 \mathrm{~km}$ from the epicenter. The earthquake was a multiple event sequence with at least 4 events recorded at $E l$ Centro in the first 25 . seconds, followed by 9 events recorded in the next 5 minutes. Clear $\mathrm{P}, \mathrm{S}$, and surface waves were observed on the strong motion record. Although the main part of the earthquake energy was released during the first 15 seconds, some of the later events were as large as $M=5.8$ and thus are important for earthquake engineering studies. The moment calculated using Fourier. analysis of surface waves agrees with the moment estimated from field measurements of fault offset after the earthquake. The earthquake engineering significance of the complex pattern of energy release is discussed. It is concluded that a cumulative increase in amplitudes of building vibration resulting from the present sequence of shocks would be significant only for structures with relatively long natural period of vibration. However, progressive weakening effects may also lead to greater damage for multiple event earthquakes.

Assessment: none

Analyses Performed: Bulletin of the Seismological Society of America $\underline{60} \cdot 137-160(1970)$

Funding: National Science Foundation grants GP 1087 and GK $1197 \mathrm{X}$

Data Availability: unknown

Archive Medium: unknown 
ERDA 02900, page 2

Contact: Mihailo D. Trifunac and James N. Brune Division of Engineering and Applied Science and Division of Geological Sciences California Institute of Technology

Pasadena, California 91109

Citations: $1.3 .2--2 \cdot 9--3 \cdot 1 \cdot 1.4 .5$ 
Model: ERDA 02907

Title: Microearthquakes: Prospecting Tool and Possible Hazard in the Development of Geothermal Resources

Date: 1972

Geographic Area: not applicable

Geographic Coordinates: not applicable

FIPS Code: not applicable

Abstract: Microearthquakes have been observed near many major geothermal areas around the world. Where detailed data are available, there is a close spatial relationship between microaarthquakes and geothermal activity. Earthquakes with magnitudes greater than about 4.5 , however, are rarely observed in geothermal areas. Locations of microearthquakes can be used to locate active faults that may channel hot water toward the surface. Earthquakes provide some risk in the development of geothermal regions since during an earthquake the flow of thermal fluid can be enhanced or slowed and structures can be damaged. Modification of reservoir fluid pressure may influence the earthquake activity.

Assessment: none

Analyses Performed: Geothermics 1 , 3-12 (1972)

Funding: National Science Foundation grant GA-1534; National Academy of Science, Arthur. L. Day Fund, grant No. 8; United Niations Purchase Order 9-20-11471.

Model Availability: in article

Archive Medium: journal article

Contact: P. L. Ward

U.S. Geological Survey

Mcnlo Park, CA 94025

Citations: $1.3 .2--2.9--3.1 .1 .4 .5$ 
Model: ERDM

Title: Project Independence: Econometric Regional Demand Model

Date: 1974

Geographic Area: USA at the census region level (9 regions)

Geographic Coordinates: not applicable

FIPS Code: all

Abstract: Model generates values of consumer energy demand for Project Independence Evaluation System (PIES), with possible fuel substitution and time variations in regional fuel mixes for residential, commercial, and industrial demand sectors. Input period: 1960-72. Prices, consumption of 52 energy products, population, per-capita income, value addcd in manufarturing, industrial production indices, Gross National Product, unemployment, demand shifts due to conservation. Forecasts for period 1975-1990.

Assessment: none

Analyses Performed: "Project Independence," Federal Energy Administration, November 1974

Funding: Federal Energy Administration

Model Availability: no use external to FEA

Archive Medium: unknown

Contact: Dr. William Hogan (202) $961-8462$

Quantitative Analysis Section

Federal Energy Administration

12th and Pennsylvania Avenue, NW

Washington, DC

Citations: $1.3 .2--2.6--3.4 .1 ; 1.3 .2--2.6--3.4 .2$ 
Data Base: ESIS

Title: Endangered Species Information System

Date: 1974

Geographic Area: National

Geographic Coordinates: not applicable

FIPS Code: all

Abstract: The data base consists of a user-accessed computerized information system for information on rare, endangered, and legally protected animal species and subspecies in the U.S. and U.S. territories. Data includes protection limits, geographic distribution (a map may be plotted automatically upon request), behavioral characteristics, habitat requirements, population structure and trends, and a reference bibliography. Only a modest introduction with computer techniques is required for the data base retrieval operation.

Assessment: none

Analyses Performed: not applicable

Funding: unknown

Data Availability: See "A User-accessed Computer Information System for Environmentally Sensitive Wild Life," E.E. Addor, and V.E. Lagarde, Lawrence Berkeley Laboratory Technical Report M-74-6, Vol. I, II, III, June 1974.

Archive Medium: unknown

Contact: .T. Miller (415) 843-2740 x6255

Mathematics and Computing Group

Lawrence Berkeley Laboratory

Berkeley, CA 94720

Master Control No. 56111

Citations: $1.3 .2--2.4--3.2 .1$ 
Model: ESMRMR

Title: Energy Supply Model for the Rocky Mountain Region

Date: 1975

Geographic Area: Rocky Mountain Region; Montana; Wyoming; Colorado; New Mexico

Geographic Coordinates: not applicable

FIPS Code: $56000,30000,08000,35000$

Abstract: This is a preliminary model of energy resource development and transportation for the Rocky Mountain region. The purpose of this model is to determine the optimal spatial and temporal development of energy resource under a variety of technical, institutional and market as sumptions. Of particular interest in the ultimale developinent of the model is the evaluation of resource utilization for various demand scenarios subject to the several policies and goals of the individual states.

The model treats a number of production areas within the Rocky Mountain Region as suppliers of various energy forms to consumption areas both inside and outside the Region. Once these energy consumption requirements have been determined, the model minimizes the cost of supplying these energy requirements subject to a set of resource, capacity, and environmental restrictions.

Asses sment: none

Analyses Performed: unknown

Funding: Energy Research and Development Administration

Model Availability: unknown

Archive Medium: unknown

Contact: James W. McFarland

Los Alamos Scientific Laboratory

P. O. Box 1663

Los Alamos, New Mexico 87545

Citations: $1.3 .2--2.6--3.3 .5 ; 1.3 .2--2.6--3.4 .2$ 
Model: ESPM

Title: The Energy Supply Planning Model

Date: 1975 August

Geographic Area: National

Geographic Coordinates: not applicable

FIPS Code: not applicable

Abstract: The Energy Supply Planning Model was designed to provide a quantitative tool which would be useful in the analysis of feasibility of candidate national energy policies in the 19751995 time frame and in their regional implications. This capability is provided by simulating the capital, labor, materials, and industrial production capabity requirements associated with the construction and operation of energy supply and energy transportation facilities needed to implement alternative energy policies.

The model simulates a highly disaggregated U.S. energy supply system including 91 types of energy extraction, processing, and transportation facilities. It determines the requirements for approximately 75 categories of capital, manpower, materials, and equipment for each region and for the nation as a whole, based on a data base containing these requirements for each facility simulated.

This model combines the development of an extensive current industrial data base and its organization into a framework which permits the policy-maker flexibility to test the sensitivity of total regional and national societal resource requirements to changes in national and regional energy policy variables. Its determination of region-specific facility schedules is useful for the evaluation of environmental implications of energy policies.

Assessment: none

Analyses Performed: unknown

Funding: National Science Foundation

Model Availability: The Energy Supply Planning Model, Vols. I and II, M. Carasso, J.M. Gallagher, et al., NTIS, Springfield, Virginia, PB 245382 and PB 245383, August 1975.

Archive Medium: hard copy 
ESPM, page 2

Contact: Meir Carasso and J. Michael Gallagher

Scientific Development Operation

Bechtel Corporation

San Francisco, CA

Citations: $1.3 .2--2.2--3.1 .2 ; 1.3 .2--2.3--3.1 .3 ; 1.3 .2--2.6--3.4 .1$;

$1.3 .2--2.8--3.4 .2$ 
Model: ETA

Title: Energy Technology Assessment

Date: 1975

Geographic Area: national

Geographic Coordinates: not applicable

FIPS Code: not applicable

Abstract: Explores some of the options by which the U.S. may move away from its present heavy dependence upon oil and gas toward a more diversified energy economy. This is accomplished by analyzing the effects of energy technologies on the costs of energy supply.

The capacity expansion process of the U.S. energy system is modeled.

Assessment: none

Analyses Performed: unknown

Funding: Electric Power Research Institute

Model Availability: unknown

Archive Medium: unknown

Contact: A.S. Manne

Harvard University

Cambridge, Massachusetts

Citations: $1.3 .2--2.6--3.4 .1$ 
Data Base: FPCBOP8

Title: Regulatory Information System

Date: 1974 October 21

Geographic Area: national by states

Geographic Coordinates: not applicable

FIPS Code: all state codes

Abstract: Federal Power Commission Bureau of Power regulations on interstate aspects of electric power industry, also licensing, construction, and operation of non-federal hydroelectric developments.

Assessment: none

Analyses Performed: unknown

Funding: Bureau of Power, Federal Power Commission

Data Availability: unknown

Archive Medium: unknown

Contact: T. A. Phillips (202) 386-6483

Chief, Bureau of Power.

Federal Power Commission

825 N. Capitol Street

Washington, D. C.

Master Control No. 70217

Citations: $1.3 .2--2.6--3 \cdot 3.5$ 
Data Base: GDB

Title: Geographic Data Base

Date: 1972

Geographic Area: National, states, standard metropolitan statistical a reas, counties; census tracts.

Geographic Coordinates: not applicable

FIPS Codes: all

Abstract: Data base consists of independent polygonal boundaries (in latitude-longitude system) with geocodes and alpha-numeric labels. Current data base includes boundaries for:

U.S. by state \& Puerto Rico \& D.C. ( 52 records)

U.S. by county \& Puerto Rico \& D.C. (3140 records)

U.S. by SMSA (275 records, 1973 definitions)

241 SMSA's (1970 definitions) by census tract $(35,000$ records $)$

Assessment: none

Analyses Performed: unknown

Funding: unknown

Data Availability: MAPEDIT Users Guide, H. Holmes, D. Austin, and W. Benson, Lawrence Berkeley Laboratory Report LBL-3072, August 1974.

CARTE Users Guide, P. Wood and D. Austin, Lawrence Berkeley Laboratory Report LBL-3073, (1975)

Archive Medium: unknown

Contact: H. Holmes (x5742), W. Benson, P. Wood (x5703), D. Austin ( $x 5313$ ) Mathematics and Computing Group (415) 843-2740

- Lawrence Berkeley Laboratory

Berkeley, CA 94720

Master Control No. 56106

Citations: $1.3 .2--2.8--3.3 .1$

$$
\text { c. }-63
$$




\section{Model: GEOCOST}

Title: A Computer Program for Geothermal Cost Analysis

Date: 1974

Geographic Area: not applicable

Geographic Coordinates: - not applicable

FIPS Code: $n$ ot applicable

Abstract: The GEOCOST computer program is a simulation model which calculates the cost of generating electricity from geothermal energy. GEOCOST will simulate the production of electricity from most types of geothermal resources. It is composed of two principal parts: a reservoir model which simulates the exploration, development, and operation of a geothermal reservoir, and a powerplant model which simulates the design, construction, and operation of the powerplant. Five different powerplant types can be simulated: flashed steam, binary fluid cycle, a hybrid combined flashed steam-binary fluid cycle, total flow, and geopressured reservoirs.

Sensitivity analyses can be performed, using the reservoir and powerplant.models, to determine the relative effect of different economic parameters, assumptions, and uncertainties on the cost of generating electricity. The GEOCOST program can be used to:

- determine:potential economic impacts of uncertainties in technology

- identify major cost components of geothermal energy

- provide a systematic method for assessing the economic potential for each type of geothermal resource and power cycle.

Combined with resource assessment information, GEOCOST can be used to define the potential supply curve (price/quantity relationship) for geothermal energy. This supply curve forms the basis for: 1) assessing the potential role of geothermal energy in competition with other sources of energy, 2) estimating potential economic incentives for new research and development programs.

GEOCOST can simulate nearly any financial and tax structure. through varying the rates of return on equity and debt, the debtequity ratios, and tax rates. The reservoir model and the powerplant model may have the same or separate financial structures and costs of capital. The plant and reservoir life can be varied over a long period, currently up to 50 years.

$$
\text { C }-64
$$


The GEOCOST program calculates the cost of energy based on the principle that the present worth of the revenues will be equal to the present worth of the expenses including investment return over the economic life of the plant and/or reservoir. The present worth factor is determined by the capital structure and rates of return on invested capital for the enterprise.

Assessment: none

Analyses Performed: GEOCOST: A Computer Program for Geothermal Cost Analysis, C. H. Bloomster, et al.; Battelle Pacific Northwest Laboratories Report, BNWL: 1888, February. 1975.

Funding: U.S. Atomic Energy Commission Contract AT(45-1):1830

Model Availability: unknown

Archive Medium: unknown

Contact: C. H. Bloomster (509) 946-2442

Information Systems

Battelle Pacific Northwest Laboratories

Richland, Washington 99352

Master Control No. 30003

Citations: 1.3.2--2.6--3.1.1.2; 1.3.2--2.6--3.1.2;

$1.3 .2--2.6--3.4 .2$ 


\section{Model: GRAPES}

Title: General Regional Assessment Program Energy Simulation

Date: 1975

Geographic Area: Pacific Northwest States; Alaska; Idaho; Montana; Oregon; Wàshington; Wyoming

Geographic Coordinates: not applicable

FIPS Code: $02000,16000,30000,41000,53000,56000$

Abstract: This is an integrated analytical as sessment program for evaluation of the potential changes which may result from various energy development or conservation scenarios.

There are three primary driving sectors: economics, energy, and demography. The dynamic results from these sectors in turn provide the primary causes of change in "driven" sectors which are: air resources, water and land resources, social and psychological impacts, health and bio-productivity. An underlying network of physical quantities (e.g. fuels and their flows) provides basis for the analytical assessment.

Assessment: none

Analyses Performed: unkingwn

Funding: ungknown

Model A vailability: unkñown

Arghive Medium: unknown

Centact: D. W. Fraley or T. P. Harrington Battelle Pa gific Northwest Laboratories PQ Bex 999

Richland, Wa shington 99352

Citations: $\quad 1,3,2==2,2==3,1,2 ; \quad 1,3,2==2,3==3,1,3$;

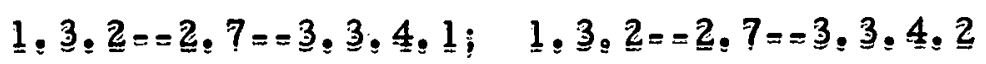


Data Base: GEOTHERM

Title: Geothermal Resources Computer File

Date: 1976

Geographic Area: global

Geographic Coordinates: not applicable

FIPS Code: not applicable

Abstract: GEOTHERM provides site-specific information on approximately 290 potential geothermal fields over the world. There are three. interrelated data files in GEOTHERM:

Geothermal Fields

Name of Field

Longitude

Users

Country

Geological Description

State

Reserve Size

County

Reservoir Properties

Latitude

Geophysical Data

Geothermal Wells

Name of Field

Geographic Information

Drilling Dates

Performance Characteristics

Depth

Flow

Temperature

Pressure

Enthalpy

Chemical Analyses

Name of Field

Geographic Information

Surface Sample Data

Well Sample Data

Water Analyses

Condensate Analyses

Residual Gas Data

GEOTHERM can be assessed by field name or geographic coordinates.

Assessment: none

$$
\text { C }-67
$$


GEOTHERM, page 2

Analyses Performed: none

Funding: U. S. Geological Survey

Data Availability: Will (late 1976-1977) be available through the University of Oklahoma

Archive Medium: unknown

Contact: James R. Swanson (703) 860-6737

GEOTHERM Project Chief,

U.S. Geological Survey

National Center M.S. 920

Reston, VA 22092

Citations: 1.3.2--2.2--3.1.1.2; 1.3.2--2.2--3.1.1.5;

$1.3 .2--2.2--3.1 .2 ; 1.3 .2--2.2--3.1 .3 ; 1.3 .2--2.3--3.1 .3$ 
Data Base: GRID

Title: National Geothermal Information Resource

Date: current

Geographic Area: national

Geographic Coordinates: not applicable

FIPS Code: not applicable

Abstract: GRID will collect and critically evaluate information related to the following major categories of geothermal science and technology: (1) resource exploration and assessment, (2) reservoir natural properties, (3) laboratory physicochemical properties," (4) reservoir development and utilization, (5) environmental effects, and (6) institutional considerations. Each of these six categories will contain both site-dependent and site-independent data collected from both domestic and foreign sources.

Bibliog raphic and numerical data will be compiled in two formats: (1) a loose-leaf handbook, and (2) computer-aided recall. The results of the comprehensive compilation will include an annotated and indexed bibliography of geothermal publications and data, critical comparisons among alternative methods of geothermal development and utilization, and recommendations for improvements in subjects requiring greater or better data collection than those currently employed.

Assessment: none

Analyses performed: unknown

Funding: U.S. Geological Survey and Lawrence Berkeley Laboratory

Data Availability: see contact

Archive Medium: magnetic tape 
GRID, page 2

Contact: S. L. Phillips (415) $843-2740, x 5818$

Energy and Environment Division

Lawrence Berkeley Laboratory

Berkeley, CA 94720

Master Control No. 56113

Citations: 1.3.2--2.1--3.1.2; 1.3.2--2.1--3.3.5; 1.3.2--2.1--3.4.1;

$1.3 .2--2.2--3.1 .1 .2 ; 1.3 .2--2.2--3.1 .1 .3 .1$;

$1.3 .2--2.2--3.1 .1 .3 .2 ; 1.3 .2--2.2--3.1 .1 .3 .3$;

1.3.2--2.2--3.1.1.3.4; 1.3.2--2.2--3.1.1.3.6;

$1.3 .2--2.2--3.1 .1 .4 .5 ; 1.3 .2--2.2--3.1 .1 .5 .1$;

$1.3 .2--2.2--3.1 .2 ; 1.3 .2--2.2--3.1 .3$;

$1.3 .2--2.2--3.2 .1 ; 1.3 .2--2.2--3.2 .2 ; 1.3 .2--2,2--3.3 .1$;

$1.3 .2--2.2--3.3 .2 ; 1.3 .2--2.2--3.3 .3 ; 1.3 .2--2.2--3.3 .4 .1$;

$1.3 .2--2.2--3.3 .5 ; 1.3 .2--2.2--3.4 .1 ; 1.3 .2--2.2--3.4 .2$;

$1.3 .2--2.3--3.1 .1 ; 1.3 .2--2.3--3.1 .2 ; 1.3 .2--2.3--3.1 .3$;

$1.3 .2--2.3--3.2 .1 ; 1.3 .2--2.3--3.2 .6 ; 1.3 .2--2.3--3.3 .1$;

$1.3 .2--2.3--3.3 .2 ; 1.3 .2--2.3--3.3 .3 ; 1.3 .2--2.3--3.3 .4 .1$;

$1.3 .2--2.3--3.3 .4 .2 ; \quad 1.3 .2--2.3--3.3 .4 .3$;

$1.3 .2--2.3--3.3 .5 ; 1.3 .2--2.3--3.4 .1$

C -70 
Data Base: GSA/GARRISON

Title: Geothermal Steam in The Geysers--Clear Lake Region, California

Date: 1972 May

Geographic Area: Pacific Coast States; California; The Geysers; Clear Lake

Geographic Coordinates: not applicable

FIPS Code: 06097,06033

Abstract: Dry, superheated natural steam is produced for the generation of electrical power from wells in the Mayacmas Mountains, north-central California Coast Ranges. The indicated source of the heat is a shallow intrusive magma body emplaced in Quaternary to Holocene time. Magmatic heat is transmitted through the largely impermeable country rock by conduction. Convectional heating takes place in stream reservoirs, predominantly zones of permeability and porosity caused by faulting and shearing. The thermal fluid is derived from. meteoric water, but some portion is probably derived from the magma. It is, at least in the exploited part of the system, in the vapor state. This controls the temperature and pressure regime, and reservoir pressures are lower than hydrostatic for similar depths in a liquid-dominated system. Generation capability was $182,000 \mathrm{kwh}$ in 1971 , and a capability of more than 600,000 . kwh is projected for 1975 .

Assessment: none

Analyses Performed: in article

- Funding: unknown

Data Availability: 'Geological Society of America 83, 1449-1468 (1972)

Archive Medium: journal article

Contact: Lowell E. Garrison

4860 Hope Lane

Sacramento, California 95821

Citations: $1.3 .2--2.2--3.1 .1 .5$ 
Data Base: HOUSE 93-29

Title: Geothermal Energy Research, Development, and Commercial Demonstration Act of 1973. Geothermal Energy Research, Development, and Demonstration Act of 1974.

Date: 1974

Geographic Area: not applicable

Geographic Coordinates: not applicable

FIPS Code: not applicable

Abstract: Hearings before the Subcommittee on Energy of the House Committee on Science and Astronautics on HR 11212 and HR 14172, held Feb. 5-11, 1974, and May 7, 1974.

Assessment: none

Analyses Performed: contained in document

Funding: U.S. House of Representatives

Data Availability: U.S. Goverment Printing Office, 32-9950

Archive Medium: hard copy

Contact: none

Citations: $\quad 1.3 .2--2.6--3.3 .5$ 
Model: HWQRB

Title: Hydraulic and Water Quality Model for River Basins

Date: 1973 January

Geographic Area: not applicable

Geographic Coordinates: not applicable

FIPS Code: not applicable

Abstract: The model is applicable to complex river basins consisting of river reaches and tributaries, vertically mixed estuaries, and stratified lakes and impoundments. It can handle both transient and steady state flow regimes and dynamic water quality conditions. Seven categories of water quality parameters are considered: (1) biochemical oxygen demand, (2) dissolved oxygen, (3) total organic carbon, (4) nitrogen, (5) phosphorus, (6) attached and floating algae, and (7) toxic compounds. Since several forms of each parameter are considered, a total of sixteen water quality constituents, including their reactions and interactions, are modeled. In addition to the quality parameters listed above, the stratified reservoir model computes vertical temperature profiles. The temperature effects on water currents and quality reactions are considered. For the rivers and estuaries portions of the model, water temperature is an input variable, at present, and can be computed by a separate program if desired.

Assessment: none

Analyses Performed: "A Dynamic Hydraulic and Water Quality Model for River Basins, "William W. Waddel, Robert G. Baca, Charles R. Cole, Albin Brandstetter, and Kenneth Feigner, Battelle Pacific Northwest

Laboratories Report BNWL-SA-4703, January 1973.

Funding: Environmental Protection Agency

Model Availability: unknown

Archive Medium: unknown 
HWQRB, page 2

Contact: Kenneth Feigner, Chief

Data Systems Branch

Region X

U.S. Environmental Protection Agency

1200 Sixth Avenue

Seattle, Washington 90101

Citations: $1.3 .2--2.2--3.1 .1 .5 .1 ; 1.3 .2--2.2--3.1 .2$ 
Model: IEES

Title: International Energy Evaluation Systems

Date: unknown

Geographic Area: global, broken down into twenty regions

Geographic Coordinates: not applicable

FIPS Code: not applicable

Abstract: Provides a framework for analyzing the world energy system. The IEES generates planning estimates depicting possible states of the world energy system, recognizing the effects of relative prices; the potential for fuel substitution, and the technological constraints that inhibit expansion of the energy system.

The world is broken into twenty geographical regions in which the supply and demand of crude oil, natural gas, and coal are modeled for each using a linear programming formulation. Transportation of fuels between regions is represented and detailed refinery and electrical generation sectors are modeled for each region. The modeling system determines equilibrium prices/quantities for final energy products as required to satisfy consumer demands determined by a separate econometric demand model.

Asses sment: none

Analyses Performed: unknown

Funding: Federal Energy Administration

Model Availability: unknown

Archive Medium: unknown

Contact: Federal Energy Administration (202) 393-6400

12 th and Pennsylvania Avenue, NW

Wa shington, DC

Citations: $1.3 .2--2.8--3.4 .2$ 


\section{Model: IGS/CARTWRIGHT}

Title: Redistribution of Geothermal Heat by a Shallow Aquifer

Date: 1971

Geographic Area: not applicable

Geographic Coordinates: not applicable

FIPS Code: not applicable

Abstract: A model for the effect of a shallow confined aquifer on the soil temperature.

Assessment: none

Analyses Performed: "Redistribution of Geothermal Heat by a Shallow Aquifer," Keros Cartwright, Geological Society of America Bulletin 82, 3197-3200. (1971)

Model Availability: unknown

Archive Medium: unknown

Contact: Keros Cartwright

Illinois Geological Survey

Urbana, Illinois 61801

Citations: $1.3 .2--2.2--3 \cdot 1.1 .2$ 
Model: IUWQI

Title: Methodology Study to Develop Evaluation Criteria for Wild and Scenic Rivers

Date: unknown

Geographic Area: not applicable

Geographic Coordinates: not applicable

FIPS Code: not applicable

Abstract: The project establishes criteria which can be used to identify and estimate economic, aesthetic, social, and other values associated with rivers. The factors which control the quality of water in a system river and the constraints necessary to maintain water quality are discussed. A method for simulating the temperature regime of a river was developed.

Assessment: none

Analyses Performed: A Methodology Study to Develop Evaluation Criteria for Wild and Scenic Rivers. Report of the Water Quality Subproject, Fred J. Watts

Funding: unknown

Model Availability: unknown

Archive Medium: unknown

Contact: Fred J. Watts,

Water Resources Research Institute

Idaho University

Moscow, Idaho

Master Control No. 43839

Citations: $1.3 .2--2.7--3.1 .2 ; 1.3 .2--2.7--3.2 .1 ; 1.3 .2--2.7--3.3 .4 .1$ 
Data Base: IU/ZEIKUS

Title: Effects of Thermal Additions from the Yellowstone Geyser Basins on the Bacteriology of the Firehole River

Date: 1972

Geographic Area: Rocky Mountain States; Wyoming; Yellowstone National Park; Firehole River

Geographic Coordinates:

FIPS Code: 56029

Abstract: A series of stations was established on the Firehole River where it flows through the main geyser basins of. Yellowstone Park, and temperature, $\mathrm{pH}$, alkalinity, conductivity, and phosphate were measured. The temperature of the water gradially increaced as it flowed linuugh the thermal area, at its warmest averaging about $16^{\circ} \mathrm{C}$ higher than in the cool area $\left(10^{\circ}\right.$ to $\left.26^{\circ} \mathrm{C}\right)$. Alkalinity, conductivity, $\mathrm{pH}$, and phosphate also increased markedly. The growth rates of periphytic bacteria were measured in situ on glass slides. The effect of temperature on the incorporation of ${ }^{14} \mathrm{C}-\mathrm{glucose}$ by benthic bacterial populations was determined.

In another study, samples of water were placed on agar media and incubated at temperatures of $25^{\circ}, 55^{\circ}$, and $70^{\circ} \mathrm{C}$ to obtain estimates of the number of bacteria able to grow at the se temperatures. The thermophilic organisms developing at $550 \mathrm{C}$ were primarily Bacillus stearothermophilus and those at $70^{\circ} \mathrm{C}$ primarily Thermus aquaticus. Thermophiles were not present in water which had not received thermal additions, but both kinds of thermophiles were found in the warm water, and numbers increased progressively as more thermal water entered. In the recovery zone downstream from the thermally heated region, the numbers of thermophilic bacteria decreased markedly.

Assessment: none

Analyses Performed: "Effects of Thermal Additions from the Yellowstone Geyser Basins on the Bacteriology of the Firehole River," J. Gregory Zeikus and Thomas D. Brock, Ecology 53, 283-290 (1972) 
IS/ZEIKUS, page?

Funding: Atomic Energy Commission (COO-1804-26)

Data Availability: unknown

Archive Medium: unknown

Contact: J. Gregory Zeikus Department of Microbiology.

or Thomas D. Brock University of Illinois Department of Bacteriology Urbana, Illinois University of Wisconsin Madison, Wisconsin 53706

Citations: $1.3 .2--2.2--3.2 .2$ 
Data Base: IVEP

Title: Imperial Valley Environmental Project

Date: 1976

Geographic Area: Pacific Coast States; California; Imperial Valley

Geographic Coordinates: not applicable.

FIPS Code: 06025

Abstract: The IVEP is a long-term project to acquire complete understanding of the environmental quality in the Imperial Valley of California prior to any major developments. The purpose of this project is to ensure that the development of geothermal resources proceeds on an environmentally sound basis. Consequently, the Imperial Valley Environmental Project (IVEP) is an intensive and comprehensive study designed to establish an environmental baseline for the Imperial Valley as well as to develop an understanding of the environmental and other effects associated with the development of geothermal resources.

The IVEP is organized into seven main study sections, which when taken together cover all the significant issues and concerns. The sections are:

1. Air Quality

2. Water Quality

3. Ecosystem Quality (soil, plants, animals, etc.)

4. Subsidence and Induced Seismicity

5. Health Effects

6. Socio-Economics

7. Integrated Assessment

A strong thrust of the project is to integ rate the output of the various studies into a single overall assessment. This includes not only the data obtained from the Lawrence Livermore Laboratory studies but input from all other identifiable programs as well. To this end, the Imperial Valley Environmental Project is developing a data base from information available through a number of previous and current studies relating to the long-term environmental assessment of the Imperial Valley.

Assessment: none 


\section{IVEP page 2}

Analyses Performed: unknown

Funding: U.S. Energy Research and Development Administration

Data Availability: See: "An Overview of the Imperial Valley Environmental Project," L.R. Anspaugh and P..L. Phelps, Lawrence Livermore Report UCID-17067, April 8, 1976.

Archive Medium: unknown

Contact: L.R. Anspaugh (415) 477-1100. 3512

Biomedical and Environmental Research Division

Lawrence Livermore Laboratory

P. O. Box 808

Livermore, California 94550

Citations: $1.3 .2--2.1--3.1 .1 .3 .6 \quad 1.3 .2--2.1--3.1 .1 .4$;

1.3.2--2.2--3.1.1.2; 1.3.2--2.2--3.1.1.3.6;

1.3.2--2.2--3.1.1.4.5; 1.3.2--2.2--3.1.1.5.1;

$1.3 .2--2.2--3.1 .2 ; 1.3 .2--2.2--3.1 .3 ; .1 .3 .2--2.2--3.2 .2$;

$1.3 .2--2.2--3.3 .1 ; \quad 1.3 .2--2.2--3.3 .2 ; 1.3 .2--2.2--3.3 .4 .1$;

$1.3 .2--2.2--3.3 .5 ; 1.3 .2--2.2--3.4 .2 ; 1.3 .2--2.3--3.1 .3$;

$1.3 .2--2 ; 3--3.2 .2 ; 1.3 .2--2.3--3.3 .1 ; 1.3 .2--2.3--3.3 .2$;

$1.3 .2--2.3--3.3 .3 ; 1.3 .2--2.3--3.3 .4 .1$;

$1.3 .2--2.3--3.3 .5 ; 1.3 .2--2.3--3.4 .1 ; 1.3 .2--2.5--3.3 .4 .1$;

$1.3 .2--2.5--3.3 .4 .3 ; 1.3 .2--2.5--3.4 .1$;

$1.3 .2=-2.6--3.3 .4 .3 ; \cdot 1.3 .2--2.6--3.3 .5 ; 1.3 .2--2.6--3.4 .1$;

$1.3 .2--2.7--3.3 .4 .1 ; 1.3 .2--2.8--3,4.2$ 
Data Base: LBL-3232

Title: Radioactivity of Geothermal Systems

Date: unknown

Geographic Area: Western States; Nevada

Geographic Coordinates: not applicable

FIPS Code: 32000

Abstract: Radioactivity anomalies associated with hot spring systems have been recognized and documented for many decades. The most observable radioelements in hot waters a re radium -226 and radon -222 , both members of the uranium -238 decay series. An examination of hot spring waters in Nevada indicates the presence of these radioelements, in varying abundances, in spring systems where $\mathrm{CaCO}_{3}$ is the predominant material being deposited. Systems where silica predominates are relatively low in radioactivity. At a hot spring site in northcentral Nevada where $\mathrm{CaCO}_{3}$ predominates, gamma-ray levels from 0.25 to 0.5 milliroentgens per hour were measured over an enclosed warm pool: At other systems where $\mathrm{SiO}_{2}$ predominates, gamma exposure rates are nearly two orders of magnitude lower.

The emanation of ${ }^{222} \mathrm{Rn}$ from thermalized fault zones is a possible exploration tool in locating a geothermal resource. An alpha-track detector survey within a potential resource area indicates anomalously high $\mathrm{Rn}$ associated with hot pools, but a rather featureless $R n$ pattern in valley alluvium away from the hot springs.

Results of studies combining neutron activation analyses of hot and cold waters in a region, and radiometric analyses of the waters and hot spring deposits, suggest that uranium entering a geothermal system is localized at depth by (1) a reducing environment afforded by $\mathrm{H}_{2} \mathrm{~S}$ and (2) precipitation of uranyl carbonate. The presence of the $U$ at a depth is indicated at the surface by its daughter products, $\mathrm{Ra}$ and $\mathrm{Rn}$, in hot spring waters and deposits.

Along with these geochemical aspects, an understanding of the radioactivity of a geothermal resource is necessary to evaluate the environmental impact of its possible future development. 
LBL-3232 page 2

Assessment: none

Analyses Performed: contained in document

Funding: U.S. Energy Research and Development Administration

Data Availability: unknown

Archive Medium: hard copy

Contact: Harold A. Wollenberg

Lawrence Berkeley Laboratory

University of California

Berkeley, CA 94720

Citations: 1.3.2--2.2--3.1.2; 1.3.2--2.2--3.1.3;

$1.3 .2--2.3--3.1 .2 ; 1.3 .2--2.3--3.1 .3$ 
Model: LEMFEU

Title: Linear Economic Model of Fuel and Energy Use in the United States

Date: unknown

Geographic Area: national

Geographic Coordinates: not applicable

FIPS Code: not applicable

Abstract: This is a linear programming model of the U.S. economy developed to investigate patterns of energy use in the United States and to examine the economic impacts of fuel and energy shortages.

The model represents lhe U.S. economy during 1972. The main components are the 1967 input-output table updated to 1972 , and a matrix of occupation coefficients and a set of energy coefficients for each industry. The input-output table contains 97 sectors, each of which has three associated activity levels: domestic output, transferred imports, and final demand. The electric utilities and iron and steel sectors are further disaggregated to show detail in the production processes; this disaggregation permits fuel substitution in these sectors. Electricity generation has been disaggregated intö coal, oil, gas, nuclear, and hydroelectric plants. Balance constraints equate total production plus imports to the total consumption by industry and final demand. The objective function represents gross national product.

Assessment: none

Analyses Performed: unknown

Funding: Electric Power Research Institute

Model Availability: unknown

Archive Medium: unknown

Contact: Peter Benenson or Roger Glassey (415) 843-2740

Lawrence Berkeley Laboratory

Berkeley, California 94720

Citations: $1.3 .2--2.6--3.4 .1$ 
Model: LIRAQ-1

Title: Livermore Regional Air Quality Model - Mode 1

Date: 1973

Geographic Area: not applicable

Geographic Coordinates: not applicable

FIPS Code: not applicable

Abstract: The LIRAQ-1 model is a regional air quality model developed for use by an air pollution control district in studying regional land use, control strategy, and air quality problems: The model treats temporally and spatially varying meteorology and source emissions, complex topography and simple physical sinks in a regional domain in the well mixed layer below the inversion. The model can be applied to variable size grids, to regions, subregions, or cities, and to various meteorological situations.

Assessment: none

Analyses Performed: in preparation

Funding: National Science Foundation

Model Availability: Livermore Regional Air Quality Model (LIRAQ-1) M. MacCracken and K. Grant, Final Report UCRL-76198, in preparation.

Archive Medium: unknown

Contact: M. MacCracken and K. Grant (415) 447-1100, x3481 Atmospheric and Geophysical Sciences Division Physics Department

Lawrence Livermore Laboratory

P. O. Box 808

Livermore, CA 94550

Master Control No. 30066

Citations: $1.3 .2--2.3--3.1 .1$ 


\section{Model: LIRAQ-2}

Title: Livermore Regional Air Quality Model (Kinetics and Transport)

Date: 1974

Geographic Area: not applicable

Geographic Coordinates: not applicable

FIPS Code: not applicable

Abstract: LIRAQ-2 is an Eulerian (fixed spatial grid)-multibox regional air quality model, incorporating mass-consistent advection; diffusion and photochemical kinetics. Using as input data the topography, meteorology, and pollutant source inventory for the region of interest, LIRAQ- 2 computes the time and spatial variations of both the ground level pollutant concentrations and the average vertical concentrations between ground level and the buttum of lle inversion layer. The model is structured in a modular fashion, with a module (submodel) for each of the major calculational steps, such as pollutant transport, chemical kinetics, and generation of mass-consistent wind fields from meteorological and topographical data.

Assessment: none

Analyses Performed: Development of an Air Pollution Model, Michael MacCracken, Second Semi-annual Report to the National Science Foundation, Feb. 27, 1974.

Funding:" National Science Foundation

Model Availability: unknown

Archive Medium: unknown

Contact: Michael MacCracken and John J. Walton (415) 447-1100, x3481 Atmospheric and Geophysical Sciences Division

Physics Department

Lawrence Livermore Laboratory

P. O. Box 808

Livermore, CA 94550

Master Control No. 30060

Citations: $1.3 .2--2.3--3.1 .1$

$$
\text { C }-86
$$


Model: MESODIFF

Title: MESODIFF

Date: unknown

Geographic area: not applicable

Geographic coordinates: not applicable

FIPS Code: not applicable

Abstract: This model constructs trajectories of puffs using pre existing wind data. Gaussian diffusion of the puffs is superimposed upon the trajectories. To model a continuous point source, puffs are emitted at a rate of about 6 per hour.

Assessment: none

Analyses performed: NOAA Technical Memo to be published

Funding: unknown

Model Availability: to be published

Archive Medium: hard copy

Contact: G. Atart

ARL/NOAA

Idaho Falls, Idaho

Master Control No. 68

Citationo: $1,3,2--2,3=-3.1 .1$ 
Model: MIROH

Title: Multi-Regional Input-Output Model, Harvard-Economic Development Administration Data Base

Date: 1973

Geographic Area: National and state

Geographic Coordinates: not applicable

FIPS Code: not applicable

Abstract: Sponsored by the Economic Development Administration (EDA) in 1970, the Harvard Economic Research Project (HERP) was undertaken to describe and analyze all the sales and purchases of every industry at a multiregional level of the economy. This was an initial attempt to study regional economic conditions rather than routine national input-output tables. The large-scale Harvard Multiregional Input-output (MIRO)project was completed with the assistance of Jack Faucett Associates and contributors from various government agencies. Tapes in the LBL data base were obtained through the Faucett As sociates and generally include 44-51 regions and 44-86 industries for the years 1947, 1958, and 1963 plus projections for the years 1970 and 1980. These data tapes have been extensively used, verified and in many instances restructured, corrected, and "filled in" by LBL.

Assessment: none

Analyses Performed: A Multi-Regional Input-Output Model for the U.S. Implementation and Evaluation of the Multiregional Model of the U.S., Esther Schroeder, Karen R. Polenske, and Albert J. Walderhaug, Harvard Economic Research Project, HERP Report 21, EDA-OER-005.

Funding: Economic Development Administration

Model Availability: unknown

Archive Medium: unknown 
MIROH, page 2

Contact: H. Ruderman and Esther Schroeder (415) 843-2740, x6292/5306 Mathematics and Computing Group

Lawrence Berkeley Laboratory

Berkeley, CA 94720

Master Control No. 56127

Citations: $1.3 .2--2.6--3.4 .1 ; 1.3 .2--2.6--3.4 .2 ; 1.3 .2 \div-2.8--3.4 .2$ 
Data Base: NAWDEX

Title: National Water Data Exchange

Date: 1976

Geographic Area: national by collection site

Geographic Coordinates: not applicable

FIPS Code: not applicable

Abstract: The primary mission of NAWDEX is to identify sources of water data and to provide the linkage between those who a acquire and those who use water data.

A variety of services will be provided by NAWDEX. Those of major significance are:

The Identification of Sources of Water Data: The Water Data Sources Directory identifies organizations that collect water data, locations within these organizations from which water data may be obtained, the geographic areas in which water data are collected by these organizations, the types of water data collected and made available, alternate sources for acquiring the organization's data, and the media in which the collected data are available.

Nationwide Indexing of Water Data: The Master Water Data Index identifies individual sites for which water data are available, the locations of these sites, the organizations collecting the data, the hydrologic disciplines for which data are available, the periods of time for which the data are available, water-data parameters available, the freguency of measurement of the parameters, and the media in which the data are available. More than 80,000 water-data sites are currently being indexed.

Data Storage and Retrieval: The NAWDEX Program Office will make an effort to arrange for this service, in special cases, through the use of data storage and retrieval facilities of NAWDEX member organizations. This service will be limited to those members having small holdings of significant data which they wish to contribute to the NAWDEX program but for which they have no suitable system for storage and retrieval. NAWDEX is currently authorized to negotiate agreements for direct access by its members, or other interested organizations, to the Daily Values File of the 
NAWDEX, page 2

National Water Data Storage and Retrieval System (WATSTORE) of the Geological Survey. This file contains large volumes of water data collected or measured on a daily schedule including streamflow values, river stages, reservoir contents, water temperatures, specific conductance values, sediment : concentrations and discharges, and ground-water levels. The Daily Values File may be used by an authorized user for retrieval purposes only or it may be used for the. storage of limited amounts of data. WATSTORE has additional data files which contain nearly 1,000,000 chemical analyses of both surface and ground waters, over 300,000 annual peak observations of streamflow and river stages, and inventory data for over 150,000 ground-water sites. While these files are not available for direct access, the data contained in these files are available through NAWDEX. Direct access to computerized water-data systems of other organizations will be negotiated by NAWDEX as the need arises.

Assessment: none

Analyses Performed: unknown

Funding: U.S. Geological Survey

Data Availability: The service capabilities of NAWDEX are supported by a nationwide network of Local Assistance Centers. These Centers have been established in offices of NAWDEX members to provide local and convenient access to NAWDEX and its services. This network has been initially established in the 46 district offices of the Geological Survey's Water Resources Division. These offices are located in 45 States and Puertin Rico. Most are equipped with computer terminals, thereby providing an extensive telecommunication network for access to the computerized directory and indexes being developed for the NAWDEX program. As the NAWDEX membership increases, additional Centers will be added in large population a reas and areas of high-user interest to provide more uptimal access to NAWDEX and its services. 
NAWDEX, page 3

Archive Medium: unknown

Contact: Melvin D. Edwards (703) 860-6031

Hydrologist

National Water Data Exchange

U.S. Geological Survey

421 National Center

Reston, Virginia 22092

Citations: 1.3.2--2.1--3.1.2; 1.3.2--2.2--3.1.2; 1.3.2--2.2--3.1.2;

$1.3 .2--2.2--3.2 .2 ; 1.3 .2--2.4--3.1 .2 ; 1.3 .2--2.4--3.2 .1$;

$1.3 .2--2.4--3.2 .2 ; 1.3 .2--2.5--3.1 ; 1.3 .2--2.5--3.2 .1$;

$1.3 .2--2.5--3.2 .2 ; 1.3 .2--2.5--3.3 .2 ; 1.3 .2--2.7--3.1 .2$;

$1,3.2--2.7--3.2 .1 ; 1.3 .2--2.7 \div-3.2 .2$ 
Data Base: NBS/DF-73-012

Title: National Bureau of Standards: Thermodynamics and Thermochemistry

Date: 1971

Geographic Area: not applicable

Geographic Coordinates: not applicable

FIPS Code: not applicable

Abstract: The substance-property index and bibliography sections of the Bulletin of The rmodynamics and Thermochemistry, no. 14 (May 1971) have been converted to a file designed for computerized searching. In this version each substanceproperty entry has been supplemented by a searching key.

Assessment: none

Analyses Performed: unknown

Funding: unknown

Date Availability: National Technical Information Service

Springfield, VA 22151

as COM $-73-10627$

Archive Medium: magnetic tape

Contact: National Bureau of Standards

Metallurgy Division

Washington, D. C.

Master Control No. 60305

Citations: $1.3 .2--2.2--3.1 .2$ 
Data Base: NIH/DF -73-002

Title: Medline: Search Capability of Articles Indexed in Index Medicus

Date: 1970 to date

Geographic Area: not applicable

Geographic Coordinates: not applicable

FIPS Code: not applicable

Abstract: An on-line bibliographic searching service is provided by the National Library of Medicine. Some 120 libraries in health-related institutions, primarily medical schools, have become part of a nationwide user network. By conversing with a computer via a typewriter-like terminal, a physician, medical librarian, or other health official is able to retrieve almost instantaneously references to the latest journal articles in his area of interest. The file contains more than 400,000 citations to articles from about 1, 200 major journals indexed for Index Medicus, or almost 60 percent of the citations printed since January. 1, 1970. Medline searches the Medlars data bases, Source documents: 1,200 major health journals.

Assessment: none

Analyses Performed: unknown

Funding: unknown

Data Availability: on-line searches only

Archive Medium: unknown

Contact: Associate Director for Library Operations (301) 656-4000 National Library of Medicine 8600 Rockville Pike

Bethesda, Maryland 20014

Master Control No. 60201

Citations: $1.3 \cdot 2--2 \cdot 7--3 \cdot 3 \cdot 4 \cdot 1$ 
Data Base: NMIMT/SUMMERS

Title: Preliminary Study of the Geochemical Characteristics of Thermal Waters in New Mexico

Date: 1972

Geographic Area: Southwestern States; New Mexico

Goegraphic Coordinates: not applicable

FIPS Code: 35000

Abstract: not available .

Assessment: none

Analyses Performed: Paper given at the 24th Session of the International Geological Congress, Section 11, Hydrogeology; Montreal, Canada, 1972. Pages 241-250 of Proceedings.

Funding:

Data Availability: unknown

Archive Medium: unknown

Contact: W.K. Summers and R. A. De.ju

New Mexico Institute of Mining and Technology

Socorro, New Mexico

Citations: $\quad 1.3 .2--2.2--3.1 .2$ 
Data Base: NOAA/CDS

Title: Climatological Data (by state)

Date: 1882 to date

Geographic Area: national by state

Geographic Coordinates: not applicable

FIPS Code: all

Abstract: Climatological Data by state, with temperature, atmospheric pressure, wind speed and direction, relative humidity, sunshine, precipitation, evaporation (later records).

Assessment: none

Analyses Performed: unknown

Funding: National Oceanic and Atmospheric Administration

Data Availability: 854 volumes of approximately 400 pages each.

Available on interlibrary loan: Free reproduction of up to 15 pages.

Archive Medium: hard copy

Contact: Chief, Atmospheric Services Library (301) 427-7919

Environmental Data Service

National Oceanic and Atmospheric Administration

8060 13th St.

Silver Spring, Maryland 20910

Master Control No. 22527000

Citations: $\quad 1.3 .2--2.7--3.1 .3$ 
Data Base: NOAA/DRS

Title: Daily River Stages

Date: 1858 to date

Geographic Area: North American rivers

Geographic Coordinates: not applicable

FIPS Code: not applicable

Abstract: Daily river stages of principal rivers in U.S. since 1890 , and of the Ohio, Mississippi, and various other rivers since 1858. Data from river gage stations. Maps, charts included.

Assessment: none

Analyses Performed: unknown

Funding: unknown

Data Availability: available through interlibrary loan

Archive Medium: 70 volumes of approximately 100 pages each

Contact: Chief, Atmospheric Sciences Library (301) 655-4000, Environmental Data Service, National Oceanic and Atmospheric Administration, 806013 th Street, Silver Spring, Maryland 20910

Master Control No. 22525000

Citations: $1.3 .2--2.2--3.1 .2$ 
Data Base: NOAA/LCS

Title: Local Climatological Summary with Comparative Data

Date: $1949-1966$

Geographic Area: national

Geographic Coordinates: not applicable

FIPS Code: not applicable

Abstract: Yearly climatological summaries (2 pages each) from over 300 stations in the U.S. and territories, covering years 1949-1966. Monthly averages and extremes for 13 standard weather parameters and general weather summaries are included.

Assessment: none

Analyses Performed: unknown

Funding: National Oceanic and Atmospheric Administration

Data Availability: on interlibrary loan

Archive Medium: 23 volumes of reports

Contact: Chief, Atmospheric Sciences Library (301) 655-4000, Environmental Data Service, National Oceanic and Atmospheric Administration, 8060 - 13 th Street, Silver Spring, Maryland 20910

Master Control No. 22536000

Citations: $1.3 .2--2.7--3 \cdot 1.3$ 
Data Base: NY/DEC 70

Title: Beneficial Uses of Thermal Discharges

Date: 1970

Geographic Area: worldwide

Geographic Coordinates: not applicable

FIPS Code: not applicable

Abstract: Proceedings of a Conference on title subject held in Albany, NY, September 17-18, 1970. Treats various beneficial uses of thermal discharges in New York, Mississippi, Tennessee, Oregon, Arizona, Washington, Pennsylvania, United Kingdom, Japan, and Iceland. These uses include agriculture, urban heating systems, and fish culture.

Assessment: none

Analyses Performed: in document

Funding: various

Data Availability: "Proceedings of the Conference on the Beneficial Uses of Thermal Discharges, "New York State Department of Environmental Conservation, Albany, New York, September 17-18, 1970

Archive Medium: hard copy

Contact: not applicable

Citations: $1.3 .2--2.2--3.1 .2$ 
Data Base: OBERS

Title: Office of Business Economics/Economic Research Service

Date: 1973

Geographic Area: national; state; standard metropolitan statistical areas

Geographic Coordinates: not applicable

FIPS Code: all

Abstract: This data base constitutes projections of economic activity and population for each of the Nation's 253 Standard Metropolitan Statistical Areas (SMSA's) and the BEA Areas of the United States. The measures include total personal income, total population, per capita income, total employment, total earnings, earnings for each of 28 industries and indexes of production for 4 mining and 15 manufacturing industry groups. They include projected data for 1975, 1980, 1985 1990,2000 , and 2020 .

Assessment: none

Analyses Performed: 1972 OBERS Projections (Series E), U.S. Bureau of Economic Analysis, Stock No. 5425-0013, U.S. Government Printing Office, April 1974

Funding: Bureau of Economic Analysis

Data Availability: available through RUSTIC or SEEDIS (q. v.)

Archive Medium: unknown

Contact: Bureau of Economic Analysis (202) 655-4000

Department of Commerce

14 th and $K$ Streets N. W.

Wa shington, D. C.

Master Control No. 56115

Citations: 1.3.2--2.6--3.3.4.3; 1.3.2--2.6--3.4.1;

$1.3 .2--2.8--3.4 .1 ; \quad 1.3 .2--2.8--3.4 .2$ 
Data Base: ORRMIS

Title: Oak Ridge Regional Modeling Information System

Date: 1974 September

Geographic Area: not applicable

Geographic Coordinates: not applicable

FIPS Code: not applicable

Abstract: A geographically oriented information system is being developed by the Regional Environmental Systems Analysis Program at Oak Ridge National Laboratory (ORNL). The system is being designed to operate either as a stand-alone inquiry-retrieval system or as a communications supervisor for use with simulation models. The primary purpose of ORRMIS is to provide the data management capability for analysis models which forecast the spatial distribution and ecological effects of activities within a geographical region. The geographic orientation of the system may, however, be replaced with a more general adjacency relationship thereby extending its use to non-geographic models.

Assessment: none

Analyses Performed: unknown

Funding: National Science Foundation RANN Program

Data Availability: unknown

Archive Medium: unknown

Contact: see: ORRMIS, Oak Ridge Regional Modeling Information System, Part I, Richard C. Durfee, Oak Ridge National Laboratory Report ORNL-NSF-EP-73, September 1974.

Citations: 1.3.2--2.4--3.2.1; 1.3.2--2.4--3.2.2; 1.3.2--2.5--3.1.1.1;

1.3.2--2.5--3.1.1.2; 1.3.2--2.5--3.3.1;

$1.3 .2--2.6--3.4 .1 ; 1.3 .2--2.6--3.4 .2$ 
Model: OSSIM

Title: Oregon State Simulation Model

Date: unknown

Geographic Area: Pacific-Northwest; Oregon

Geographic Coordinates: not applicable

FIPS Code: 41000

Abstract: Models socioeconomic activity for the state of Oregon, forecasting over a 50 year period (1970-2020) the effect. on various components caused by varying others. Electrical energy consumption by industrial, residential, commercial, transportation sectors. Air pollution, stàte taxes shown.

Assessment: none

Analyses Performed: unknown

Funding: Rockefeller Foundation

Model Availability: unknown

Archive Medium: unknown

Contact: Chris Calligan (503) 754-3664

Willamette Simulation Unit

Oregon State University

Corvallis, Oregon

Citations: $1.3 .2--2.5--3.3 .1 ; 1.3 .2--2.6--3.1 .3 ; 1.3 .2--2.6--3.3 .1$;

$1.3 .2--2.6--3.4 .1 ; 1.3 .2--2.8--3.1 .3 ; 1.3 .2--2.8--3.3 .1$;

$1.3 .2--2.8--3.4 .1 ; 1.3 .2--2.8--3.4 .2$ 
Model: OSU/BODVARSSON

Title: Thermal Problems in the Siting of Reinjection Wells

Date: 1972

Geographic Area: not applicable

Geographic Coordinates: not applicable

FIPS Code: not applicable

Abstract: This paper presents a theoretical discussion of the thermal problems involved in the disposal of flash water from geothermal power plants by reinjection. The basic equations for the subsurface temperature field in the reinjection zone are derived both for rocks and intergranular and fracture flow. The extent of the thermal contamination by the reinjected water is discussed. In the case of a continuous mass flow of flash water of $1000 \mathrm{~kg} / \mathrm{sec}$ for a period of 25 years, the contamination may reach out to as much as 5 kilometers or more from the point of re-entry, depending on the type of rock involved.

Assessment: none

Analyses Performed: Geothermics 1, 63-66 (1972).

Funding: National Science Foundation grant GA-25896

Model Availability: in article

Archive Medium: journal article

Contact: G. Bodvarsson

Dept. of Oceanog raphy

Oregon State University

Corvallis, OR 97331

Citations: $1.3 .2--2.2--3.1 .1 .5 .1$ 
Model: PANS

Title: A Program to Tally Population by Annuli and Sectors

Date: 1972 October

Geographic Area: not applicable

Geographic Coordinates: not applicable

FIPS Code: not applicable

Abstract: A computer program to tally two point-wise functions by sectors and annuli located about a set of arbitrary points on the surface of a sphere has been written in FORTRAN for the IBM 360 computer. It was originally written to study populations in the vicinity of reactor sites.

The program requires the latitudes and longitudes of the set of points, or sites, about which the tallies are to be performed as well as the radii of the annuli and the number of sectors. The principal sector is centered on north, and the annuli must be sufficiently small to neglect the curvature of the sphere. The point-wise functions must be supplied with the latitude and longitude of each point.

The program produces (1) a summary of the incremental and cumulative tallies in optional forms, (2) a summary of points out of range, (3) a summary of points tallied with the bearing and distance of each from its site(s).

Assessment: none

Analyses Performed: unknown

Funding: U.S. Atomic Energy Commission

Model Availability: PANS: A Program to Tally Population by Annuli and Sectors, P.R. Coleman and A. A. Brooks, Oak Ridge National Laboratory Report, ORNL-TM-3923, October 1972.

Archive Medium: hard copy

Contact: P.R. Coleman and A. A. Brooks Oak Ridge National Laboratory

Oak Ridge, Tenn. 37830

Citations: $\quad 1.3 .2--2.6--3.4 .1$

$$
\text { C - } 104
$$


Data Base: PARAMETRIX

Title: The Effects of Geothermal Energy Utilization on Stream Biota and Water Quality at The Geysers, California

Date: 1975

Geographic Area: Pacific Coast States; California; The Geysers

Geographic Coordinates: not applicable

FIPS Code: 06097

Abstract: 13 -month study of the effects of present operational practices at The Geysers geothermal field on the aquatic insect and fish populations. Insect populations are normal as are fish except for rainbow/steelhead trout in the Big Sulphur Creek. This exception is attributed to naturally occurring fumaroles venting nitrites into the Creek.

Assessment: none

Analyses Performed: contained in document (75-0731-040FR)

Funding: Union Oil Company, Geothermal Division

Data Availability: in document

Archive Medium: hard copy

Contact: Dr. Richard S. LeGore

Environmental Services Section

Parametrix, Inc.

4122 Stone Way North

Seattle, Washington 98103

Citations: $1.3 .2--2.4--3.1 .2 ; 1.3 .2--2.4--3.2 .1 ; 1.3 .2--2.4--3.2 .2$ 
Model: PERCOL

Title: One-dimensional Model of the Movement of Trace Radioactive Solute Through Soil Columns

Date: 1972

Geographic. Area: not applicable

Geographic Coordinates: not applicable

FIPS Code: not applicable

Abstract: A one-dimensional mathematical model, PERCOL, has been developed to predict the movement of radionuclides through porous media as a function of measurable chemical parameters of the media. Laboratory column studies were conducted to verify the model. System parameters considered include eoil type, radionuclide type, waste composition, flow rate, column length, and soil saturation: The agreement between measured radionuclide movement and that predicted by the model is considered good.

Assessment: none

Analyses Performed: One-Dimensional Model of the Movement of Trace Radioactive Solute Through Soil Columns: The PERCOL Mode1, R. C. Routs on and R. J. Serne, Battelle Pacific Northwest Laboratories Report BNW L-1 718, 1972.

Experimental Methods for Obtaining PERCOL: Model Input and Verification Data, R. J. Serne, R. C. Routson, and D. A. Cochran, Battelle Pacific Northwest Laboratories Report BNWL-1721, 1973.

Funding: U. S. Atomic Energy Commission Contract AT(45-1)-2130

Model Availability: unknown

Archive Medium: unknown 
PERCOL, page 2

Contact: R. C. Routson and R.J. Serne

Water and Land Resources Department

Battelle Pacific Northwest Laboratories

Richland, Washington 99352

Master Control No. 40241 .

Citations: 1.3.2--2.1--3.1.1; 1.3.2--2.2--3.1.1.5.1;

$1.3 .2--2.2--3.1 .2$ 
Model: PIES

Title: Project Independence Evaluation System

Date: 1974

Geographic Area: national

Geographic Coordinates: not applicable

FIPS Code: not applicable

Abstract: Forecasts energy supply; demand, prices, and capital requirements for determining energy policy. Includes all major energy sources - - solar, geothermal, coal and derivatives, gas oil, nuclear, hydroelectric power.

Assessment : none

Analyses Performed: Project Independence, Federal Energy Administration, November 1974

Funding: Federal Energy Administration

Model Availability: no use external to FEA

Archive Medium: unknown

Contact: Dr. William Hogan (202) $961-8462$

Quantitative Analysis Section

Federal Energy Administration

12 th and Pennsylvania

Washington, D. C.

Citations: $1.3 .2--2.6--3.4 .1 ; 1.3 .2--2.6--3.4 .2 ; 1.3 .2--2.8--3.4 .1$;

$1.3 .2--2.8--3.4 .2$ 


\section{MODEL: PILOT}

Title: The Stanford Energy Model

Date: 1975

Geographic Area: national

Geographic Coordinates: not applicable

FIPS Code: not applicable

Abstract: Describes in physical terms the technological interactions within and across the sectors of the economy. The general objective of the model is to determine, in the face of a changing energy picture, what the U.S. could achieve in physical terms over a sufficiently long time horizon.

Assessment: none

Analyses Performed: "Optimization Modeling for Energy Policy Analysis, (Stanford PILOT Energy Mode1)," G. B. Dantzig and S.C. Parikh; Operations Research Department, Stanfo'rd University, SOL 76-1, February 1976.

Funding: Electric Power Research Institute

Model Availability: unknown

Archive Medium: unknown

Contact: G.B. Dantzig, T. Connolly, S. Parikh, L. Lasdon, or M. Avriel

Stanford University

Palo Alto, California

Citations: $1.3 .2--2.6--3.4 .1 ; 1.3 .2--2.6--3.4 .2$ 
Data Base: PL 91-581

Title: Federal Geothermal Steam Act of 1970

Date: 1970

Geographic Area: not applicable

Geographic Coordinates: not applicable

FIPS Code: not applicable

Abstract: Federal regulatory legislation for exploitation of geothermal energy.

Assessment: not applicable

Analyses Performed: not applicable

Funding: U.S. Government

Data Availability: U. S. Government Printing Office, Washington, D. C.

Archive Medium: hard copy

Contact: not applicable

Citations: $\quad 1.3 .2--2.5--3.3$ 
Model: PREVIEWS.

Title: PREVIEWS 85 Integrated Economic Forecasting and Simulator's Model

Date: 1970

Geographic Area: national

Geographic Coordinates: not applicable

FIPS Code: not applicable

Abstract: Investigates potentialities of the U.S. economy as a function expected of final demands for industrial outputs, foreign competition and technological changes in products and processes. Energy supplies, demands and technologies are provided in detail.

All U.S. economic activity is described in terms of a complex Leontief input-output model. Final demands a re either endogenous or exogenous; in the former case demographic and family-behaviors are exogenous inputs to dynamic submodels. Intermediate relations (embodied in technologically changing direct coefficients) are endogenous. Model has detailed energy sectors and can be constrained by resource, labor, and capacity availabilities. A wide variety of demand, supply, policy, price, and/or technology scenarios can be simulated for impact analyses.

Assessment: none

Analyses Performed: unknown

Funding: unknnwn

Model Availability: unknown

Archive Medium: unknown

Contact: W. Holder Fisher

Battelle Memorial Institute

Columbus, Ohio

Citations: $\quad 1.3 .2--2.6--3.4 .1$ 
Model: PSRV

Title: Pseudo Relative Velocity Spectra

Date: 1968

Geographic Area: not applicable

Geographic Coordinates: not applicable

FIPS Code: not applicable

Abstract: A program for computing seismic time histories. It generates a seismic response spectrum which represents a second order; single degree of freedom system expressed by La Place transformation.

Assessment: none

Analyses Performed: Design Earthquake and Response Spectra, W. W. Hays, Earthquake Information Bulletin, July-August 1974.

Funding: U.S. Geological Survey

Model Availability: unknown

Archive Medium: unknown

Contact: R. B. Park (303) 234-4041

Seismicity and Risk Analysis Branch

U.S. Geological Survey

Denver Federal Center (D1)

Denver, Colorado 80225

Master Control No. 30274

Citations: $1.3 .2--2.8--3.1 .1 .4 .5 ; 1.3 .2--2.9--3.1 .1 .4 .1$ 
Data Base: RUSTIC

Title: Regional and Urban Studies Information Center

Date: 1974

Geographic Area: National, states, counties

Geographic Coordinates: not applicable

FIPS Code: all

Abstract: RUSTIC is a machine-readable collection of socio-economic data bases. These include census data, geographic data, Dual Independent Máp Encoding (DIME) files, OBER's Projections, population characteristics, and economic files. For a complete listing see: "Regional and Urban Studies Socioeconomic Data Bases at Oak Ridge National Laboratory,"!. A.S. Loeb1, D.F. Burch, and N.S. Malthouse, Oak Ridge Report ORNL-TM-5182, November 1975.

Asses sment: none

Analyses Performed: unknown

Funding: Department of Housing and Urban Development, U.S. Energy Research and Development Administration

Data Availability:

I. RUSTIC handles data requests on a cost-recovery basis. Cost estimates are provided by the RUSTIC staff.

II. U ser requests must be submitted in written form. They should outline data requirements in terms of content, geographical level and fürmat, and reference the cost estimate.

III. Consultation to aid the user in determining his specific data needs is available from the RUSTIC staff.

Archive Medium: magnetic tape 
RUSTIC, page 2

Contact: Andrew S. Loebl or Deborah F. Burch (615) 483-8611

Ext. 3-0311 or 3-0353

RUSTIC

Oak Ridge National Laboratory

P. O. Box X

Oak Ridge, TN 37830

Master Control No. 56201

Citations: $1.3 .2--2.6--3.4 .1$ 
Model: SDNM

Title: Systems Dynamics National Model

Date: unknown

Geographic Area: national

Geographic Coordinates: not applicable

FIPS Code: not applicable

Abstract: This model establishes long-term national socioeconomic goals, explains causes of socioeconomic behavior, and is used to develop policies for dealing with interconnected national problems. It lists 14 production/distribution activities including resources, energy, transportation, each including 10 production factors, capital, labor, etc.

Assessment: none

Analyses Performed: unknown

Funding: unknown

Model Availability: unknown

Archive Medium: unknown

Contact: Alfred P. Sloan School of Industrial Management Massachusetts Institute of Technology

Cambridge, Massachusetts

Citations: $1.3 .2--2.6--3.4 .1 ; 1,3.2--2.6--3.4,2$ 
Model: SEAS

Title: Strategic Environmental Assessment System

Date: 1974 January

Geographic Area: National

Geographic Coordinates: not appliçable

FIPS Code: not a pplicable

Abstract: SEAS is a collection of interdependent models to assess the future economic and environmental consequences of federal pollution control policies. It is a system of special-purpose models linked to a model of the U.S. economy. The specialpurpose models are:

National Economic Modules-based on the University of Maryland's INFURUM together witli a national macroeconometric model of Chase Econometrics Associates.

Materials Flow Modules--SOLIDWASTE es timates the annual tonnage of non-industrial solid waste, the expected disposal methods, and their associated costs. STOCK provides information on raw material sources, reserve levels, and relative production costs.

National Residuals Generation Module--RESGEN applies pollutant residual coefficients.

Transportation Modules--forecast demand for vehicle travel for passenger and freight purposes.

Energy Forecas Module--forecasts energy use by fuel type.

Experimental Regional Modules--regionalize data.

Assessment: none

Analyses Performed: unknown

Funding: Environmental Protection Agency

Model availability: unknown

Archive Medium: unknown 
SEAS, page 2

Contact: Cal Lawrence

Office of Research and Development

Environmental Protection Agency

Washington, D. C. 20460

Citations: $\quad 1.3 .2--2.6--3.4 .2$ 
Data Base: SEEDIS

Title: Socio-Economic Environmental-Demographic. Information System

Date: $1975 \mathrm{March}$

Geographic Area: National, states, counties, standard metropolitan areas, census tracts.

Geographic Coordinates: not applicable

FIPS Code: all

Abstract: SEEDIS is a collection of data bases on demography, social science, geography, business enterprise, employment, labor force, earnings, transportation, agriculture, health, environment, natural resources, and physics.

Assessment: none

Analyses Performed: unknown

Funding: U.S. Energy Research and Development Administration, Contract W-7.405-ENG-48

Data Availability: see. An Overview of the LBL Socio-EconomicEnvironmental-Demographic Information System, Donald M. Austin, Suzanne G. Kranz, and Carl Quong. Lawrence Berkeley Laboratory Report LBL-3699, March 1975 .

Archive Medium: IBM 1360 Photodigital Chipstore

Contact: Donald M. Austin (415) 843-2740, x5313 Mathematics and Computing Group Lawrence Berkeley Laboratory Berkeley, CA 94720

Citations: $1.3 .2--2.6--3.4 .1$ 
Data Base: SEN 93-GR

Title: Geothermal Resources

Date: 1973

Geographic Area: not applicable

Geographic Coordinates: not applicable

FIPS Code: not applicable

Abstract: Hearings before the Subcommittee on Water and Power Resources of the Senate Committee on Interior and Insular Affairs, held June 13, 1973, and August 10-11, 1973.

Assessment: none

Analyses Performed: contained in document

Funding: U.S. Senate

Data Availability: U. S. Government Printing Office, $21-4380$ and $26-4730$

Archive Medium: hard copy

Contact: none

Citations: $\quad 1.3 .2--2.6--3.3 .5$ 
Model: SITE

Title: A Methodology for Assessment of Energy Facility Siting Patterns

Date: 1975 August

Geographic Area: not applicable

Geographic Coordinates: not applicable

FIPS Code: not applicable

Abstract: SITE methodology has been developed for evaluating health, environmental, and socioeconomic impacts related to utilization of alternate sites for energy production within a region of interest. The cost, impact, and attribute vectors, which a re generated and displayed on density maps, can be used in a multiparameter overlay process to identify preferable siting areas. The assessment of clustered facilities in energy centers is also possible within SITE analysis framework. An application of the SITE methodology to Northern Illinois is presented. Also included is a description of the on-going extension of SITE for the accumulative evaluation of alternative regional energy siting patterns and fuel cycle options. An appendix provides documentation and user information for the SITE computer program.

Assessment: none

Analyses Performed: SITE: A Methodology for assessment of energy facility siting patterns, N.A. Frigerio, L.J. Habeggar, et al., Argonne National Laboratory Report, ANL/AA-2, August 1975.

Funding: U.S. Energy Research and Development Administration, Contract.W-31-109-Eng-38.

Model availability: unknown

Archive Medium: unknown

Contact: L.J. Habegger or L.S. Hoover (312) 739-7711, x5139

Energy and Environmental Systems Division

Argonne National Laboratory

9700 S. Cass Avenue

Argonne, Illinois 60439

Citations: $1.3 .2--2,8--3.3 .1$

$$
\text { C }-120
$$


Model: SMICK

Title: Scavenging Model Incorporating Chemical Kinetics

Date: 1973

Geographic Area: not applicable

Geographic Coordinates: not applicable

FIPS Code: not applicable

Abstract: SMICK calculates ground level concentrations of from one to ten species in non-vertical rain which has passed through a pollutant plume, and optionally, the cross-plume washout rate. As originally written; washout of $\mathrm{SO}_{2}$ and $\mathrm{HSO}_{3}$ from. a buoyant gaussian plume is considered, assuming $\mathrm{SO}_{2}$ absorbtion to be limited either by gas or liquid phase diffusion. In another application, the Scott and Hobbs mechanism is considered under similar circumstances. The input data required include raindrop sizes and distribution, meteorological variables, stack and plume data, and background concentrations of species being considered. A card reader and a line printer are the only input/output devices required.

Assessment: none

Analyses Performed: Natural precipitation washout of sulfur compounds from plumes, M. T. Dana, J. M. Hales, W. G. N. Slinn, and M. A. Wolf, EPA-R3-73-047, June 1973.

Funding: unknown

Model Availability: unknown

Archive Medium: unknown

Contact: D. R. Drewes (509) $946-2890$

Atmospheric Sciences Dept. Battelle - Pacific Northwest Laboratory Battelle Blvd.

Richland, Washington 99352

Master Control No. 30002

Citations: $1.3 .2--2.3--3.1 .1 ; 1.3 .2--2.3--3.1 .2$ 


\section{Model: SRIEM}

Title: Stanford Research Institute Enèrgy Model

Date: 1974

Geographic Area: national

Geographic Coordinates: not applicable

FIPS Code: not applicable

Abstract: The SRI National energy model is a comprehensive model of U.S. energy markets covering all major fuels from primary resources to end use (usable) energy consumption over the period of 1975 to 2025 . The model is regionally disaggregated with eight demand regions--such as New England--and 20 primary resource supply regions--such as Alaska-North Slope--and 600 transportation and distribution links. Conversion technologies such as coal gasification, crude oil refining, and electric power generation by type of fuel are explicitly modeled. The model computes regional prices, and quantities (flows) of primary resources, products, and technology requirements over the period of 1975 to 2025 by balancing supply and demand.

Assessment: none

Analyses Performed: "A Comparative Analysis of Methodologies Used in Large Scale Modeling," Edward G. Cazalet, et al, Stanford Research Institute Report

Funding: Gulf Oil Company and Stanford Research Institute

Model Availability: unknown

Archive Medium: unknown

Contact: Edward G. Cazalet

Manager, Division Analysis

Stanford Research Institute

333 Ravenswood Avenue

Menlo Park, California 94025 
SRIEM, page 2

$$
\begin{aligned}
\text { Citations: } & 1.3 .2--2.6--3.1 .1 .2 ; 1.3 .2--2.6--3.3 .1 ; \\
& 1.3 .2--2.6--3.3 .4 .3 ; 1.3 .2--2.6--3.3 .5 ; \\
& 1.3 .2--2.6--3.4 .1 ; 1.3 .2--2.6--3.4 .2 ; \\
& 1.3 .2--2.8--3.3 .1 ; 1.3 .2--2.8--3.3 .5 ; \\
& 1.3 .2--2.8--3.4 .1 ; 1.3 .2--2.8--3.4 .2
\end{aligned}
$$


Data Base: STORET

Title: Storage and Retrieval of Water Quality and Point Source Data

Data: current

Geographic Area: national except California, Georgia, Illinois, Michigan, New York, and Texas

Geographic Coordinates: not applicable

FIPS Code: not applicable

Abstract: River, lake, and estuary data from EPA, USGS, USA Corps of Engineers, U.S. Forestry Service, TVA, 42 states, dating from 1964. 180, 000 locations listed under 1,600 parameters classed in 17 major groups, station and sample identifiers. Biological data a subset, BIOSTORET.

Assessment: none

Analyses performed: unknown

Funding: Environmental Protection Agency

Data Availability: through EPA

Archive Medium: computer disc files

Contact: Charles S. Conger (202) 426-7792

Chief, User Assistence and Information Access Branch

Monitoring and Data Support Division

Office of Air and Water Programs

Environmental Protection Agency

Washington, D. C. 20460

Master Control No. 56371

Citations: 1.3.2--2.1--3.1.2; 1.3.2--2.2--3.1.1.5.1;

1.3.2--2.2--3.1.2; 1.3.2--2.2--3.2.1;

$1.3 .2--2.2--3.2 .2 ; 1.3 .2--2.3--3.1 .2$

1.3.2--2.4--3.1.2; 1.3.2--2.7--3.1.2 
Model: SUWQI

Title: Syracuse University. Water Quality Index

Date: unknown

Geographic Area: not applicable

Geographic Coordinates: not applicable

FIPS Code: not applicable

Abstract: Provides a water pollution index or an index for a single pollutant which can be used as measures of water quality, or in conjunction with a figure for the dollar benefit of a pure water supply, used to display graphically. the dollar benefit to be realized at any level of water pollution.

Assessment: none

Analyses Performed: unknown

Funding: unknown

Mondel Availability: unknown

Archive Medium: unknown

Contact: Syracuse University

Syracuse, New York

Citations: $1.3 .2--2.1--3.1 .1 ; 1.3 .2--2.1--3.1 .2$;

$1.3 .2--2.2--3.1 .2 ; 1.3 .2--2.5--3.1$;

$1.3 .2=-2.6--3.1 .2 ; 1.3 .2--2.7--3.1 .2$ 
Data Base: SWIRS

Title: Solid Waste Information Retrieval System

Date: 1967

Geographic Area: international

Geographic Coordinates: not applicable

FIPS Code: not applicable

Abstract: Provides a comprehensive reference to published international literature on solid waste management to speed technological transfer. Abstracts with author and subject indexes are published monthly. Solid Waste Information Retrieval Systems (SWIRS) also offers general inquiry, literature search, technical translations and user seminar services. Federal, state, and local agencies in the United States and abroad, as well as members of the general research and development community, consulting engineers, attorneys, students and others of the lay public, use these services to further knowledge of new techniques and principles, and to keep abreast of current research projects.

Assessment: none

Analyses Performed: unknown

Funding: Environmental Protection Agency

Data Availability: through EPA

Archive Medium: unknown

Contact: John A. Connolly

Technical Information Officer

Technical Information Staff

Environmental Protection Agency

Master Control No. 56370

Citations: 1.3.2--2.1--3.1.1.3.4; 1.3.2--2.1--3.3.1;

$1.3 .2--2.1--3.4 .2$ 
Model: TAMU/WHITING

Title: Possible Effects of Geothermal Water and Stream Production on the Subsurface Environment

Date: 1974

Geographic Area: not applicable

Geographic Coordinates: not applicable

FIPS Code: not applicable

Abstract: not available

Assessment: none

Analyses Performed: American Institute of Chemical Engineers Symposium Series 70, No. 136, 762-771 (1974).

Funding :

Model Availability:

Archive Medium:

Ciontact: R. L. Whiting

Texas A\&M University

College Station, Texas

Citations: $1.3 .2--2.2--3.1 .1 .3 .6 ; 1.3 .2--2.9--3.1 .1 .4 .5$ 
Data Base: TRIS

Title: Transportation Research Information System

Date: unknown

Geographic Area: not applicable

Geographic Coordinates: not applicable

FIPS Code: not applicable

Abstract: A on-line, data base of: technical abstracts, resumes of on-going work, inventory of statistical data bases, profiles of simulation and decision models; directory of relevant information services.

Assessitient: none

Analyses Performed: unknown

Funding: Department of Transportation

Data Availability: unknown

Archive Medium: unknown

Contact: Office of the Secretary (202) 426-4000

Department of $T$ ransportation

4007 th St. S. W.

Wa shington, D. C.

Master Control No. 70173

Citations: $1.3 .2--2.8--3.4 .2$ 
Data Base: TRRP

Title: Air Quality Monitoring: and T rends Report

Date: to 1972

Geographic Area: national

Geographic Coordinates: not applicable

FIPS Code: not applicable

Abstract: Provide quarterly and yearly air quality monitoring and trends reports. Provides air studies and trends analysis upon request also. Periodic reports produced determine which dat a re relevant to problems.. Selects subset data. Performs statistical analysis upon data. Twenty programs and software packages are used, e.g. SAS, Box-Jenkins, Mark II, SPSS.

Assessment: none

Analysies Performed: unknown

Funding: Environmental Protection Agency

Data Availability: unknown

Archive Meduim: unknown

Contact: William M. Cox and John B. Clark

Monitoring and Reports Branch

Environmental Protection Agency

Master Control No. 56362

Citations: $1.3 \cdot 2--2 \cdot 3--3 \cdot 1.3$ 
Data Base: UCR/COMBS

Title: Microearthquake Investigation of the Mesa Geothermal Anomaly, Imperial Valley, California

Date: 1973 November

Geographic Area: Pacific Coast States; California; Imperial Valley

Geographic Coordinates:

FIPS Code: 06025

Abstract: not available

Assessment: none

Analyses Performed: Paper given at 1973 Fall Meeting of the American Geophysical Union, San Francisco, CA, 1973

December 10-13: EOS, Transactions of the American Geophysical Union 54, 1213-14 (1973)

Funding:

Data Availability:

Archive Medium:

Contact: J. Combs and D. M. Hadley University of California at Riverside Riverside, CA

Citations: $\quad 1.3 .2--2.9--3 \cdot 1.1 .4 .5$ 
Model: UH/HOUSTON

Title: Regional Model for Energy Analyses

Date: 1976

Geographic Area: Gulf Coast States; Texas; Houston-Galveston Standard Metropolitan Statistical Area

Geographic Coordinates: not applicable

FIPS Code: $48167,48039,48157,48201,48291,48339,48473$

Abstract: Develops a structural model of the Houston-Galveston SMSA as a tool for longrun analysis of the impact of public policy on employment, income, and migration.

The Houston Economy - non-manufacturing industry is represented at the one-digit S. I. C. level, manufacturing at the two-digit S.I.C. level. Employment, wages, and output are predicted by industry. Income is predicted by functional source. More detailed predictions are made in the energy producing and consuming sector, including refining output and inputs, feeds back consumption, drilling activity and export and inport flows. Energy demand is modelled by class of customer and type of fuel.

Assessment: none

Analyses Performed: unknown

Funding: unknown

Model Availability: unknown

Archive Medium: unknown

Contact: R.W. Thomas or G. Schuler

University of Houston

Houston, Texas

Cltations: $1.3 .2--2.6--3.4 .1$ 
Model: UH/INDUSTRY

Title: NSF Industry Studies

Date: 1975

Geographic Area: National; regional

Geographic Coordinates: not applicable

FIPS Code: not applicable

Abstract: Evaluates the effects of currently scheduled, increasingly restrictive standards for waste discharges to the water and air by the petroleum refining, chemicals, and electric power generation industries on the domestic energy production, use, and prices of oil, natural gas, and coal.

The energy system of the United States from resource extraction to final use is represented by three major components: (1) domestic production of oil and natural gas, (2) conversion of primary energy resources into fuels, electricity, chemicals, rubber, fibers, and plastics; and (3) consumption of fuels, electricity and non-energy products in the residential and commercial, industrial, and transportation sectors.

Assessment: none

Analyses Performed: unknown

Funding: National Science Foundation

Model Availability: unknown

Archive Medium: unknown

Contact: R.G. Thompson, J.A. Calloway, R.J. Lievano, or F. D. Singleton University of Houston Houston, Texas

Citations: $1.3 .2--2.3--3.3 .5 ; 1.3 .2--2.6--3.3 .5 ; 1.3 .2--2.6--3.4 .2$ 
Data Base: ULUPF

Tiele: Urban Land Use Planning File

Date: 1970

Geographic Area: National by census tract and standard metropolitan statistical area

Geographic Coordinates: not applicable

FIPS Code: all

Abstract: Contains 1970 population in group quarters, 1970 total population, total area, total water area, total land area, total non-residential land area, and total institutional land area in acres for each tract in the file. The file includes data on population density and land use/classification, of the approximately 35,000 1970 census tracts in 240 standard metropolitan statistical areas. Information is tabulated providing total land area of the census tract, total water area, land presently unavailable for residential development, area of special population concentrations and total population.

Assessment: none

Analyses Performed: unknown

Funding: unknown

Data Available: Available through RUSTIC (q. v.)

Archive Medium: magnetic tape

Contact: Andrew S. Loebl or Deborah F. Burch (615) 483-8611 x30311 RUSTIC

Oak Ridge National Laboratory

P.O. Box X

Dak Ridge, TN $37830^{\circ}$

Master Control No. 56225

Citations: $1.3 .2--2.6--3.3 .1$ 
Data Base: UM/SMITH

Title: Acute and Chronic Toxicity of Hydrogen Sulfide to the Fathead Minnow, Pimephales promelas

Date: 1976 June

Geographic Area: not applicable

Geographic Coordinates: not applicable

FIPS Code: not applicable

Abstract: Toxic effects of molecular hydrogen sulfide to eggs, fry, and juveniles of the fathead minnow, Pimephales promelas Rafinesque, were determined in flow-through bioassays. The $96-\mathrm{h}$ LC50 of laboratory-cultured juveniles at $20^{\circ} \mathrm{C}$ was $0.0243 \mathrm{mg} / 1 . \mathrm{H}_{2} \mathrm{~S}$. Fry were approximately twice as sensitive. Response with temperature in wild stock juveniles varied from a $96-\mathrm{h} \mathrm{LC50}$ of $0.7754 \mathrm{mg} / 1$. at $6.5^{\circ} \mathrm{C}$ to $0.0423 \mathrm{mg} / 1 . \mathrm{H}_{2} \mathrm{~S}$ at $25^{\circ} \mathrm{C}$. In chronic exposure to $\mathrm{H}_{2} \mathrm{~S}$ from egg through two generations of laboratory-cultured stock in flow-through bioassays, adverse effects on growth, survival, and fecundity occurred above $0.004 \mathrm{mg} / 1 . \mathrm{H}_{2} \mathrm{~S}$. Chronic exposure of wild stock up to 346 days caused adverse effects on growth and survival above $0.008 \mathrm{mg} / 1 . \mathrm{H}_{2} \mathrm{~S}$. At comparable temperatures apparent safe levels with long exposure were five - to sevenfold lower than the 96-h LC50 for both stocks.

Assessment: none

Analyses Performed: Environmental Science and Technology 10 , $565-568(1976)$

Funding: U.S. EPA Grant 800992, University of Minnesota Agricultural Experiment Station

Data Availability: unknown

Archive Medium: journal article

Contact: Lloyd L. Smith, Jr., Donavon M. Oseid, and Larry E. Olson Department of Entomology, Fisheries, and Wildlife

University of Minnesota

St. Paul, Minne sota 55108

Citations: $1.3 .2--2.2--3.2 .1 ; 1.3 .2--2.3--3.2 .1$ 
Model: UMWQI

Title: University of Michigan Water Quality Index

Date: unknown

Geographic Area: not applicable

Geographic Coordinates: not applicable

FIPS Code: not applicable

Abstract: Multi-attribute scaling procedures were applied to a nonlaboratory problem - - to the measurement of water quality. Several different variables (such as nitrate, fecal coliforms, turbidity and dissolved solids) contribute to water pollution. It is possible to obtain physical measurements of each of the variables, but no physical model exists for combining the measures into an overall index of quality. The multiattribute scaling procedures we re applied to this task by assessing, from water quality engineers, judgments about which variables should be included in the index, the type for combining the variables; the relative importance weights of the different variables, and a curve describing the functional relation between water quality and each variable. Water quality indices were obtained for two specific purposes -- for "public water supply" and for "fish and wild-life". The experimenter used a modified Delphi procedure for obtaining consensus among the engineers for each of the indices.

Assessment: none

Analyses Performed: The Application of Multi-Attribute Scaling Procedures to the Development of Indices of Value, Technical Report of the University of Michigan Engineering Psychology Laboratory

Funding: unknown

Model Availability: unknown

Archive Medium: unknown 
UMWQI, page 2

Contact: Michael F. O'Connor

Engineering Psychology Laboratory

University of Michigan

Ann Arbor, Michigan

Master Control No. 44367.

Citations: $1.3 .2--2.7--3.1 .2 ; 1.3 .2--2.7--3.2 .1 ; 1.3 .2--2.7--3.3 .4 .1$ 
Model: USDA/DF-73-019

Title: Soil Conservation Service: Flood Damage Program

Date: unknown

Geographic Area: not applicable

Geographic Coordinates: not applicable

FIPS Code: not applicable

Abstract: ECON2 Computer program computes flood damage for given stage and area, plots damage frequency.

Assessment: none

Analyses Performed: unknown

Funding: U.S. Department of Agriculture

Data Availability: National Technical Information Service Computer Products Office

Springfield, VA 22151

Archive Medium: 1 reel 9-track, 1600 bpi, odd parity magnetic tape

Contact: Soil Conservation Service. (202) 447-4543

U.S. Department of Agriculture Independence Avenue

Wa shington, D. C.

Master Control No. 60359

Citations: $1.3 .2--2.2--3 \cdot 1.1 .4 .1$ 
Model: USDA/DF-73-020

Title: Soil Conservation Service: Flood Routing

Date: unknown

Geographic Area: not a pplicable

Goegraphic Coordinates: not applicable

FIPS Code: not applicable

Abstract: A program which will flood route any frequency storm through various user-specified hydrologic networks. Detailed hydrographs and summary output reports are optionally requested.

Assessment: none

Analyses Performed: unknown

Funding: unknown

Model Availability: National Technical Information Service

Computer Products Office

Springfield, VA 22151

Archive Medium: magnetic tape

Contact: Soil Conservation Service (202) 447-4543

Department of Agriculture

Independence Avenue

Washington, D.C.

Master Control No. 60322

Citations: $\quad 1.3 .2--2.2--3.1 .1 .4 .1$ 
Model: USGS/FOURNIER

Title: Geochemical Indicators of Subsurface Temperature

Date: 1974 May-June

Geographic Area: Western States; Wyoming; Yellowstone National Park

Geographic Coordinates: not applicable

FIPS Code: 56029

Abstract: The chemical and isotopic compositions of hot-spring water and gas are used to estimate subsurface temperatures. The basic assumptions inherent in the methods are seldom stipulated. These assumptions include (1) a temperature-dependent reaction at depth, (2) a supply of the solid phase involved in the reaction to permit saturation of the constituent used for geothermometry, (3) water-rock equilibrium at depth, (4) negligible re-equilibration as the water flows to the surface, and (5) no dilution or mixing of hot and cold water. The first three assumptions are probably good for a few reactions that occur in many places. The last two assumptions probably are not.valid. for many hot-spring systems; information obtained is therefore for the shallower parts of those systems, or a limiting temperature (generally a minimum) is indicated.

The water in many warm springs with large rates of flow consists of mixtures of hot water that has come from depth and of shallow cold water. Under favorable conditions the original temperature of the hot water and the fraction of the cold water in the mixture can be estimated by using the measured temperature and silica content of the warm spring water and the temperature and silica content of non-thermal wate $r$ in the region. The method has been applied with apparent success at Yellowstone National Park.

Assessment: none

Analyses Performed: Journal of Research of the IT.S. Geological Survey 2, $259-262$ and $263-270$ (1974)

Funding: U.S. Geological Survey

Model Availability: unknown

Archive Medium: unknown 
USGS/FOURNIER, page 2

Contact: R.O. Fournier, D. E. White, and A.H. Truesdell U.S. Geological Survey

Menlo Park, California 94025

Citations: $1.3 .2--2.2--3.1 .1 .2$ 
Data Base: USGS GEOMAPS

Title: United States Geological Survey Geologic Maps

Geographic Area: entire US

Geographic Coordinates: all

FIPS Code: not applicable

Abstract: Topography plus surficial geologic features, color coded stratigraphic formations, some structural features. Scales vary for different regions.

Assessment: not applicable

Analyses Performed: not applicable

Funding: U.S. Geological Survey

Data Availability: National Cartographic Information Center U.S. Geological Survey

National Headquarters

Room IC 402

Reston, Virginia 22092

Archive Medium: maps

Contact: U.S. Geological Survey

National Headquarters

Reston, Virginia 22052

Citations: 1.3.2--2.1--3.1.1.2; 1.3.2--2.1--3.1.1.4.5 
Data Base: USGS/HAMILTON

Title: Microearthquakes at The Geysers Geothermal Area, California

Date: 1972 April 10

Geographic Area: Pacific Coast States; California; The Geysers

Geographic Coordinates: $38^{\circ} \mathrm{N}$ Lat; $122^{\circ} \mathrm{W}$ Long.

FIPS Code: 06097

Abstract: Microearthquakes in The Geysers area of northern California were recorded for 3 weeks with a radio-telemetry array of eight seismograph stations in order to examine their distribution with respect to the area developed for geothermal power. Locations were determined for 53 earthquakes within about $10 \mathrm{~km}$ of The Geysers. Most epicenters lie in a zone about $4 \mathrm{~km}$ long and $1 \mathrm{~km}$ wide passing through the geothermal field along a principal fault zone. Focal depths in this trend range from near surface to about $4 \mathrm{~km}$. A composite faultplane solution indicates dextral strike-slip faulting on a NNWstriking plane subparallel with the regional fault pattern. The results of this study suggest that accurate mapping of microearthquakes can be useful in the exploration for geothermal resources, if the earthquakes studied here are not somehow caused by development of the field.

Assessment: none

Analyses Performed: Journal of Geophysical Research 77, 2081-2086 (1972)

Funding: U.S. Geological Survey

Data Availability: unknown

Archive Medium: journal article

Contact: R.M. Hamilton and L.J.P. Muffler

U.S. Geological Survey

Menlo Park, CA 94025

Citations: $\quad 1.3 .2--2.9--3.1 .1 .4 .5$ 
Mode1: USGS/HELM

Title: One-dimensional Simulation of Aquifer System Compaction near Pixley, California

Date: 1975 June

Geographic Area: Pacific Coast States; California; San Joaquin Valley

Geographic Coordinates: not applicable

FIPS Code: 06107

Abstract: Aggregate one-dimensional compaction (consolidation) of a series of aquitards in a compacting aquifer system has been simulated through use of a finite difference represen tation of the vertical stress distribution within an idealized aquitard. Among the parameters affecting the simulated compaction are two storage coefficients (compressibility values), one for recoverable and the other for nonrecoverable compression. The se two storage coefficients introduce a transient heterogeneity within an aquitard that is generally ignored by hydrologists. A computer program with two sets of constant coefficients calculates the daily deformation due to observed changes in applied stress near Pixley, California. Although water levels fluctuate annually, no long-term water level decline occurred near Pixley between January 1, 1959 and February 4, 1971. During this period, $3.19 \mathrm{ft}(0.972 \mathrm{~m})$ of compaction was observed. The net difference between simulated and observed compaction on February 4,1971, was $1.3 \%$ of the observed value. Maximum deviation occurred in mid1964 and equaled $7 \%$ of the observed compaction.

Assessment: none

Analyses Performed: Water Resources Research 11, 465-478 (1975)

Funding: U.S. Geological Survey

Model Availability: unknown

Archive Medium: unknown 
USGS/HELM, page 2

Contact: Donald C. Helm U.S. Geological Survey Sacramento, CA 95825

Citations: $\quad 1.3 .2--2.2--3.1 .1 .3 .6$ 
Model: USGS/NATHENSON

Title: Flashing Flow in Hot Water Geothermal Wells

Date: 1973 November

Geographic Area: not applicable

Geographic Coordinates: not applicable

FIPS Code:' not applicable

Abstract: not available

Assessment: none

Analyses Performed: paper given at the 1973 Fall Meeting of the American Geophysical Union, San Francisco, CA, 1973 December 10-13: EOS, Transactions of the American Geophysical Union 54, 1215 (1973)

Funding: U.S. Geological Survey

Data Availability:

Archive Medium:

Contact: M. Nathenson

U.S. Geological Survey

Mcnlo Park, CA 94025

Citations: $1.3,2--2.2--3 \cdot 1.2$ 


\section{Data Base: USGS TOPOMAPS}

Title: United States Geological Survey Topographic Maps

Geographic Area: entire U.S. in 7.5-minute quadrangles

Geographic Coordinates: all

FIPS Code: not applicable

Abstract: Field and aerial survey quadrangles bounded by latitude and longitude lines, named for unique features within area, showing waterways, woodlands, constructions, contours. Some show roads, urban areas, public lands. Scales available, one part to 24,$000 ; 31,680 ; 62500$;

63,360 (Alaska); 125,000; 250,000; 1,000,000

Assessment: not applicable

Analyses Performed: not applicable

Funding: U.S. Geological Survey

Data Availability: National Cartographic Information Center U.S. Geological Survey

National Headquarters - Room IC 402

Reston, Virginia 22092

Archive Medium: Maps

Contact: U.S. Geological Survey

National Headquarters

Reston, Virginia 22092

Citations: $1.3 \cdot 2--2 \cdot 1--3.1 .1 .1$ 
Model: USGS/TRUESDELL

Title: The Calculation of Aquifer Chemistry in Hot-water Geothermal Systems

Date: 1974 May-June

Geographic Area: not applicable

Geographic Coordinates: not applicable

FIPS Code: not applicable

Abstract: The temperature and chemical conditions ( $\mathrm{pH}$, gas pressure, and ion activities) in a geothermal aquifer supplying a producing bore can be calculated from the enthalpy of the total fluid (liquid + vapor) produced and chemical analyses of water and steam separated and collected at known pressures. Alternatively, if a single water phase exists in the aquifer, the complete analysis (including gases) of a sample collected from the aquifer by a downhole sampler is sufficient to determine the aquifer chemistry without a measured value of the enthalpy. The assumptions made are that the fluid is produced from a single aquifer and is homogeneous in enthalpy and chemical composition. These calculations of aquifer chemistry involving large amounts of ancillary information and many iterations require computer methods.

Assessment: none

Analyses Performed: Journal of Research of the U.S. Geological Survey 2, $271-278$ (1974)

Funding: U.S. Geological Survey

Model Availability: PL/1 program from NITS, PB-219376

Archive medium: hard copy.

Contact: Alfred $\mathrm{H}$ 。 Truesdell and Wendy Singers

U.S. Geoluyical Survey

Menlo Park, Califormia 94025

Citations: $1.3 .2--2.2--3.1 .1 .5$ 
Data Base: USGS/WILLEY

Title: Geochemistry of Thermal Waters in Long Valley, California

Date: 1973 November

Geographic Area: Pacific Coast States; California; Long Valley

Geographic Coordinates: not applicable

FIPS Code: 06051

Abstract: not available

Assessment: none

Analyses Performed: Paper given at 1973 Fall Meeting of the American

Geophysical Union, San Francisco, CA, 1973

December 10-13: EOS, Transactions of the

American Geophysical Union 54, 1212 (1973)

Funding:

Data Availability:

Archive Medium:

Contact: L.M. Willey, J. B. Rapp, and I. Barnes

Citations: $\quad 1,3,2--2,2--3,1,2$ 
Data Base: USGS/YOUNG

Title: Geothermal Investigations in Idaho. I. Geochemistry and Geologic Setting of Selected Thermal Waters

Date: 1973

Geographic Area: Pacific Northwest States; Idaho

Geographic Coordinates: not applicable

FIPS Code: 16000

Abstract: not available

Asses sment: none

Analyses Performed: Water Information Bulletin of the Idaho Department of Water Administration, No. 30, 1-43 (1975)

Funding: U.S. Geological Survey

Data Availability:

Archive Medium:

Contact: H.W. Young and J. C. Mitchell

U.S. Geological Survey

Menlo Park, .CA 94025

Citations: $\quad 1.3 .2--2.2--3.1 .2$ 
Data Base: VTN

Title: A Report on the Peregrine Falcon in the Geysers Area, California

Date: 1975 August 8

Geographic Area: Pacific Coast States; California; The Geysers

Geographic Coordinates: not applicable

FIPS Code: 06097,06033

Abstract: A review of the literature on the Peregrine Falcon and 30 -hours of field observations at The Geysers area were carried.out to determine the falcon's status in the area. Geothermal energy developments were assessed with respect to their influences on the falcon.

Assessment: none

Analyses Performed: contained in document

Funding: Union Oil Company of California, Geothermal Division

Data Availability: in document

Archive Medium: hard copy

Contact: Dr. Kenneth E. Stager

Senior Curator of Ornithology

Los Angeles County Museum of Natural History

Los Angeles, CA

or

Fred Proby

VTN Consolidated, Inc.

2301. Campus Drive

Irvine, CA

Citations: $1.3 .2--2.4--3.2 .1$ 
Model: WEFM

Title: The Wharton Economic Forecasting Mode1

Date: unknown

Geographic Area: unknown

Geographic Coordinates: not applicable

FIPS Code: unknown

Abstract: The structure of the new Wharton-EFU model is explained and some of its properties described. This model was developed from the original Wharton model and the Evans model and combines the best features of both models while having some new features all of its own.

Assessment: none

Analyses Performed: unknown

Funding: unknown

Model Availability: unknown

Archive Medium: Book

Contact: M. K. Evans and L. R. Klein

Master Control No. 40448

Citations: $\quad 1.3 .2--2.6--3.4 .1$ 
Model: WQELR

Title: A Generalized Water Quality Model for Eutrophic Lakes and Reservoirs

Date: 1974 November

Geographic Area: not applicable

Geographic Coordinates: not applicable

FIPS Code: not applicable

Abstract: The models provide a detailed portrayal of the dynamic processes which determine the trophic states in lakes and reservoirs. The models a re formulated in terms of several key environmental variables: dissolved oxygen, biochemical oxygen demand, coliform bacteria, toxic material, algal populations and nutrient malerials, and the major controlling factors: light, temperature, stream flows, and loading rates.

Assessment: none

Analyses Performed: "A Generalized Water Quálity Model for Eutrophic Lakes and Reservoirs," R. G. Baca, M. W. Lorenzen, R. D. Mudd, and L. V. Kimmel, Battelle-Pacific Northwest Laboratories Report, November 1974.

Funding: Environmental Protection Agency

Model Availability: unknown

Archive Medium: unknown

Contact: R. G. Baca, M. W. Lorenzen, R. D. Mudd, or L. V. Kimmel Battelle-Pacific Northwest Laboratories, P. O. Box 997

Richland, Washington 99352

Citations: $1.3 .2--2.2--3.1 .1 .5 .1 ; 1.3 .2--2.2--3.1 .2$ 